FHWA/IN/JTRP-2001/19

\author{
Final Report
}

\title{
NOISE CONTROL AND SPEECH INTELLIGIBILITY IMPROVEMENT OF A TOLL PLAZA
}

\author{
Luc Mongeau \\ Robert J. Bernhard \\ Jeffrey P. Feist
}

November 2001 
Final Report

FHWA/IN/JTRP-2001/19

\title{
NOISE CONTROL AND SPEECH INTELLIGIBILITY IMPROVEMENT OF A TOLL PLAZA
}

\author{
By \\ Prof. Luc Mongeau \\ Associate Professor \\ Principal Investigator \\ and \\ Prof. Robert J. Bernhard \\ Professor \\ Principal Investigator \\ and \\ Jeffrey P. Feist \\ Research Assistant \\ School of Mechanical Engineering \\ Purdue University \\ Joint Transportation Research Program \\ Project Number: C-36-67DDD \\ File Number: 9-10-55 \\ SPR-2414 \\ Conducted in Cooperation with the \\ Indiana Department of Transportation \\ and the \\ U. S. Department of Transportation \\ Federal Highway Administration
}

The contents of this report reflect the views of the authors, who are responsible for the facts and the accuracy of the data presented herein. The contents do not necessarily reflect the official views or policies of the Indiana Department of Transportation of the Federal Highway Administration at the time of publication. The report does not constitute a standard, specification, or regulation.

Purdue University

West Lafayette, IN 47907

November 2001 
TECHNICAL REPORT STANDARD TITLE PAGE

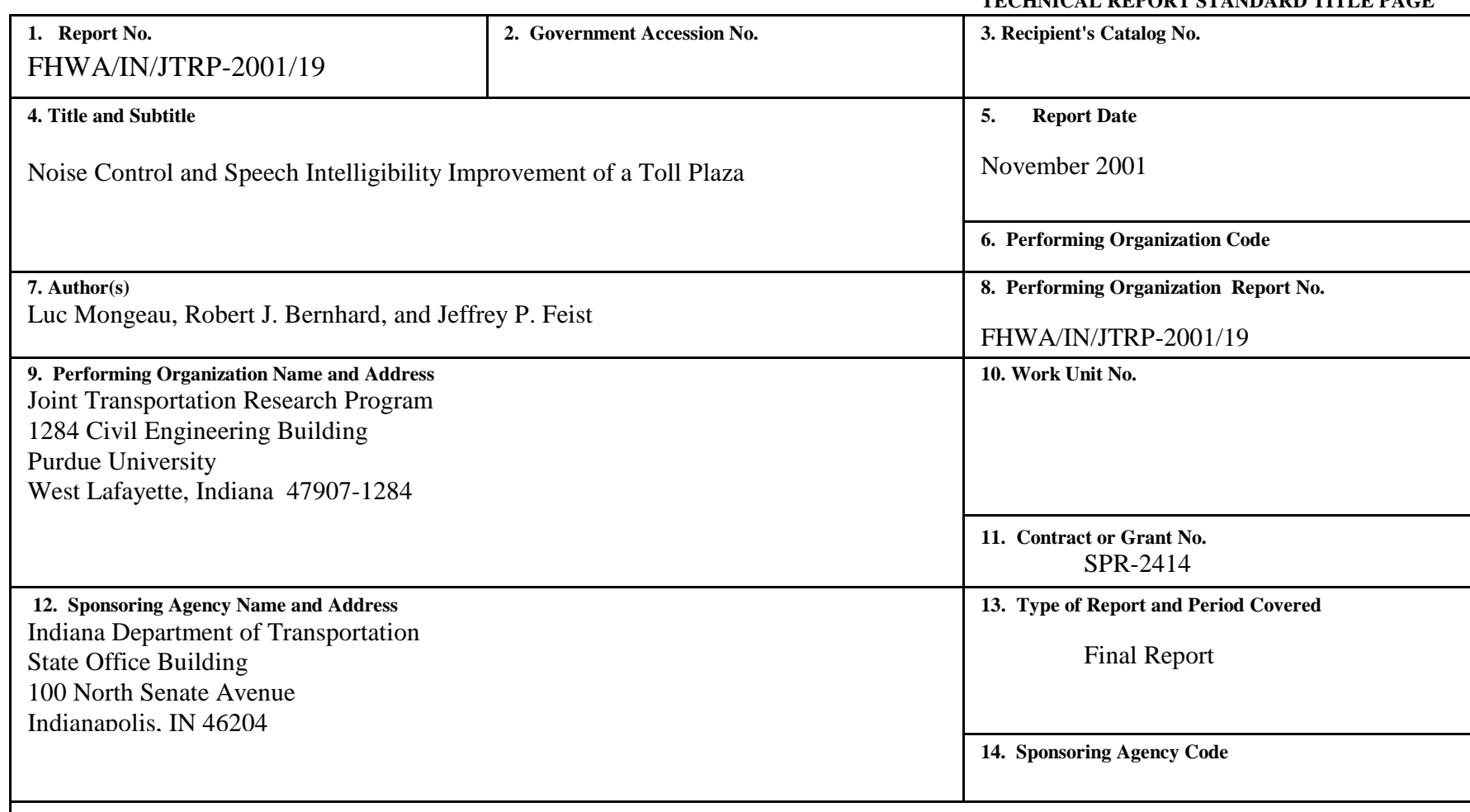

\section{Supplementary Notes}

Prepared in cooperation with the Indiana Department of Transportation and Federal Highway Administration.

\section{Abstract}

Vehicular toll roads are one component of many municipal transportation systems. Open communication windows, often used in tollbooths, facilitate essential communication and monetary transactions. However, the vehicle noise generated outside the booth is easily transmitted into the booth via the open window. Personnel working at toll collection plazas are exposed to extended, continuous traffic noise. Sustained noise levels of this nature may cause hearing loss, induce fatigue or stress, and reduce worker productivity. The annoyance and discomfort related to continuous noise exposure may create an unpleasant working condition and may affect the hospitality of the tollbooth operators and their attitude toward customers. Furthermore, the noise level may hinder communication with customers and may compromise safety. Reduction of the noise level and an improvement in speech intelligibility are highly desirable.

The acoustics of a typical toll plaza and structural noise control strategies were modeled using a beam tracing technique. Noise control strategies involved the application sound absorbing material to the overhead canopy, the construction of sound absorbing partial barriers, and the treatment of tollbooth walls with sound absorbing material. In terms of noise control, the results suggest that the direct field is more important that the reflected field.

The effects of active noise control (ANC) systems to reduce traffic noise and improve speech intelligibility at the toll plaza was investigated. The ANC systems included a range of headsets and a prototype external unit designed to create a local region of attenuation. Significant noise reduction can be achieved with a sealed, closed ear ANC headsets. However, the various systems seemed to have little positive effect upon speech intelligibility under traffic noise conditions. The result imply that the signal to noise ratio under toll plaza conditions is poor and that level overloading effects may further reduce intelligibility.

Altered systems were modeled to improve the signal to noise ratio and reduce the noise level. The improved systems utilize a directional microphone and a sealed ANC headset. With a high order directional microphone, good speech intelligibility is achievable even in the presence of toll plaza vehicle noise.

17. Key Words
Toll plaza, toll road, active noise control, speech intelligibility,
beam tracing.
beam tracing.

\section{Distribution Statement}

No restrictions. This document is available to the public through the National Technical Information Service, Springfield, VA 22161

\begin{tabular}{|c|c|c|c|}
\hline $\begin{array}{c}\text { 19. Security Classif. (of this report) } \\
\text { Unclassified }\end{array}$ & 20. Security Classif. (of this page) & 21. No. of Pages & 237 \\
\hline
\end{tabular}




\section{TECHNICAL Summary}

INDOT Research

Technology Transfer and Project Implementation Information

TRB Subject Code: 17-4 Noise and Air Quality Studies

Publication No.: FHWA/IN/JTRP-2001/19, SPR-2414

November 2001

Final Report

\section{NOISE CONTROL AND SPEECH INTELLIGIBILITY IMPROVEMENT OF A TOLL PLAZA}

\section{Introduction}

Vehicular toll roads are one component of many municipal transportation systems. Personnel working at toll collection plazas are exposed to extended, continuous traffic noise. Sustained noise levels of this nature may cause hearing loss, induce fatigue or stress, and reduce worker productivity. The annoyance and discomfort related to continuous noise exposure may create an unpleasant working condition and may affect the hospitality of the tollbooth operators and their attitude toward customers. Furthermore, the noise level may hinder communication with customers and may compromise safety. Reduction of the noise level and an improvement in speech intelligibility are highly desirable.

Open communication windows, often used in tollbooths, facilitate essential communication and monetary transactions. Typical transactions between toll collectors and patrons involve charge cards, commuter cards, toll tickets, and the exchange of cash. Direct visual and physical contact between patrons and attendants is made possible by the open communication window. One disadvantage is that vehicle noise generated outside the booth is easily transmitted into the booth via the open window. The booth structure acts as a partially open enclosure, which may amplify road noise due to sound reflections off of rigid surfaces and reverberation within the enclosure.

The objective of this research is to lower the noise levels experienced by toll collection workers and increase the speech intelligibility of the tollbooth environment.

\section{Findings}

Contributions of the study include the following:

1. Surveys of toll plaza employees confirm that traffic noise creates an unpleasant work environment and frequently hampers communication.

2. A toll plaza was modeled using a beam tracing method. Toll plaza model predictions indicate that implementation

of reasonable passive noise control solutions will reduce the sound level by approximately $3 \mathrm{~dB}$. This is a noticeable but rather modest decrease. Results suggest that significant noise reductions can only be achieved by reducing the direct sound field.

3. Active noise control (ANC) systems were investigated to control the direct field. The potential of ANC systems to 
reduce low frequency sounds within reverberant, partially open enclosures has been demonstrated in previous studies. Improvements in speech intelligibility may be possible when dominant low frequency sounds in traffic noise mask speech signals and reduce intelligibility.

4. The noise attenuation of ANC systems was investigated. The noise reduction ratings (NRR) for the ANC systems were highly dependent upon the system configurations. A sealed ANC headset achieved a NRR value of $24 \mathrm{~dB}$, while some of the open systems achieved NRR values near $0 \mathrm{~dB}$. Passive components, such as circumaural earcups, result in the greater attenuation found in closed headset.

5. The speech intelligibility at the toll plaza was investigated using standardized methods. Traffic noise conditions at the toll plaza were recorded. In the presence of traffic noise, the speech intelligibility was poor even for the highest vocal efforts.
6. The effects of active noise control systems on speech intelligibility were investigated. While some of the systems offer significant attenuation of noise, none of the systems improved the speech intelligibility significantly. The systems are not adaptive and do not directly alter the signal to noise ratio. The results suggest that low frequency traffic noise masking of higher frequency communication sounds is not as detrimental as in-band masking. The best systems had the greatest overall reduction, which suggests that overloading effects are important.

7. Innovative systems were modeled to improve intelligibility at the toll plaza. A sealed ANC headset with a dipole directional microphone input and level reduction provides $20 \%$ more of the speech cues to a listener under high vocal efforts. A higher order directional microphone input, creates a good communication situation $(75 \%$ and greater of the speech cues available to the listener).

\section{Implementation}

Predictions of the performance of a hypothetical modified ANC system resulted in a significant increase in speech intelligibility. The next step is to build a number of prototype systems. The systems would be built to further investigate performance. In addition to the directional microphones and single channel feedback active noise control algorithms, exploration of adaptive noise control algorithms would be beneficial. Adaptive algorithms produce different system transfer functions for different signals. By selectively attenuating noise and passing speech, the signal to noise ratio could be further increased. Investigation into cost and comfort issues should be addressed.
The toll plaza model demonstrated that passive noise control solutions, which address the reflected sound field, do not substantially reduce the noise level. The modeling approach did not account for coherent reflections that cause reverberation and resonance. Due to the statistically random nature of vehicle noise, it is a reasonable approximation to use a source model assuming incoherent waves where phase is neglected. If resonance conditions occur at the toll plaza, placement of absorbing material may have a more dramatic effect than predicted by the model. It is suggested that the possible existence of resonance conditions be investigated in a subsequent study. On-site measurements at many locations throughout the toll plaza 
could be used to investigate the presence of standing waves. The results of the beam tracing method could be validated through on-site measurements.

\section{Contacts}

For more information:

Prof. Luc Mongeau

Principal Investigator

Purdue University

1077 Herrick Laboratories

West Lafayette, IN 47907-1077

Phone: 765-494-9342

Fax: $\quad 765-494-0787$

Prof. Robert J. Bernhard

Principal Investigator

Purdue University

1077 Herrick Laboratories

West Lafayette, IN 47907-1077

Phone: 765-494-2141

Fax: $\quad 765-494-0787$
Indiana Department of Transportation

Division of Research

1205 Montgomery Street

P.O. Box 2279

West Lafayette, IN 47906

Phone: 765-463-1521

Fax: $\quad 765-497-1665$

\section{Purdue University}

Joint Transportation Research Program

School of Civil Engineering

West Lafayette, IN 47907-1284

Phone: 765-494-9310

Fax: 765-496-1105 


\section{TABLE OF CONTENTS}

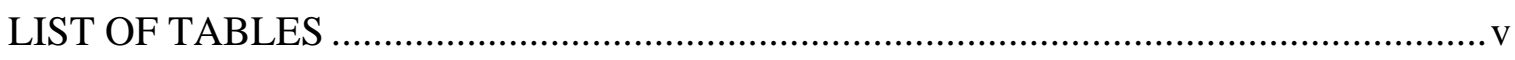

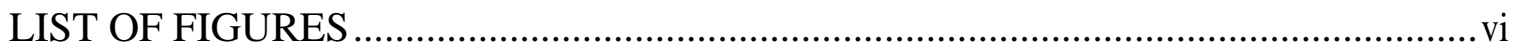

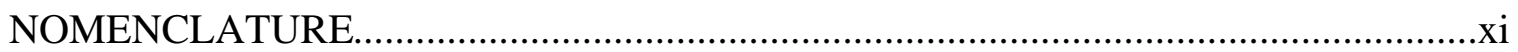

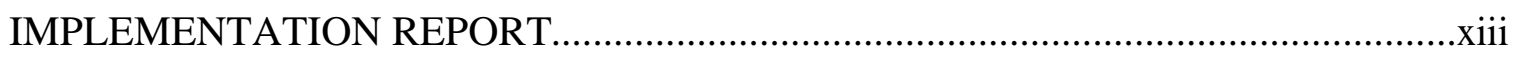

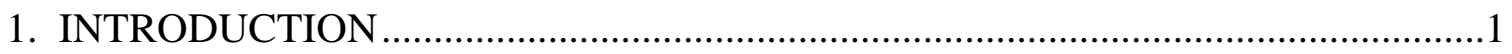

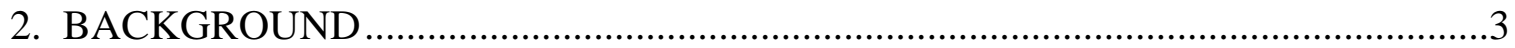

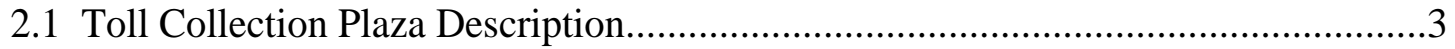

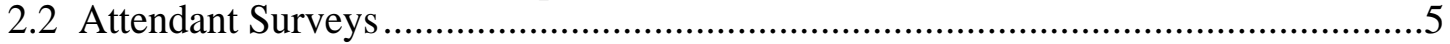

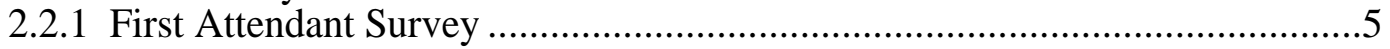

2.2.2 Second Attendant Survey ............................................................... 7

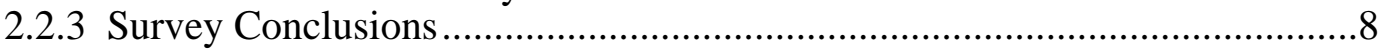

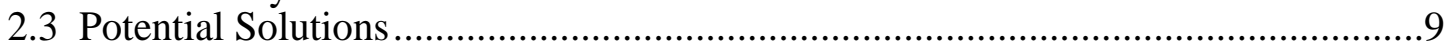

2.3.1 Passive Noise Control Techniques...........................................................

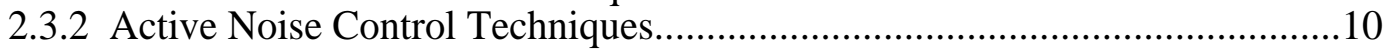

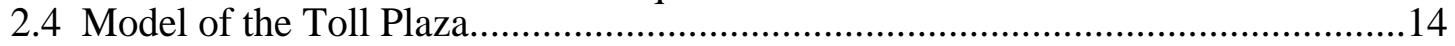

2.4.1 The LMS RAYNOISE Software Package Theory ................................... 15

2.4.1.1 Mirror Image Source Method ...........................................................16

2.4.1.2 Ray Tracing Method ...................................................................... 17

2.4.1.3 Beam Tracing Method .................................................................. 18

2.4.1.4 LMS RAYNOISE Diffraction Technique ........................................19

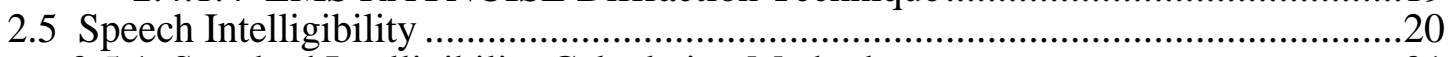

2.5.1 Standard Intelligibility Calculation Methods ......................................... 21

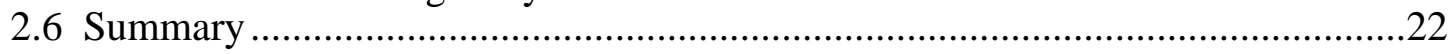

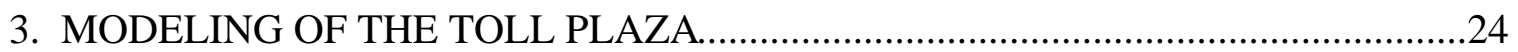

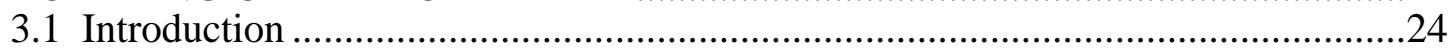

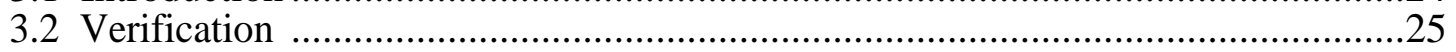

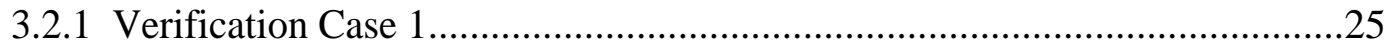

3.2.2 Verification Case 2 ..........................................................................2

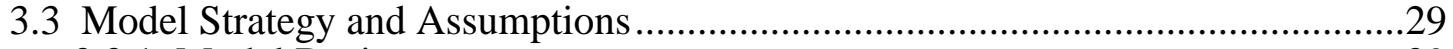

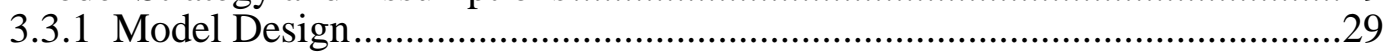

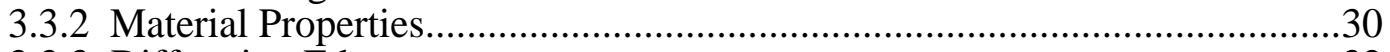

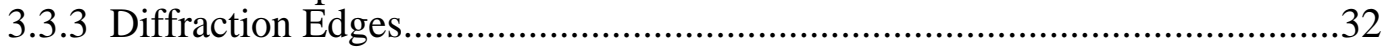

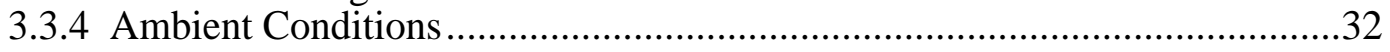

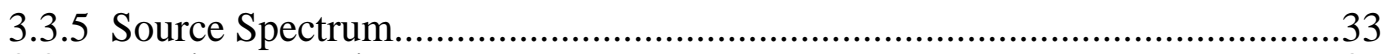

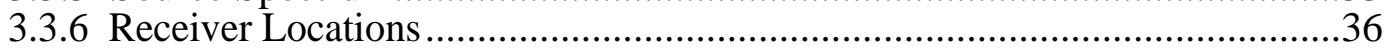




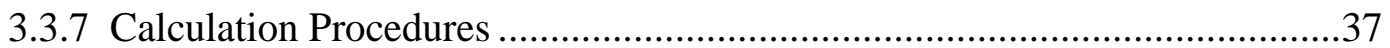

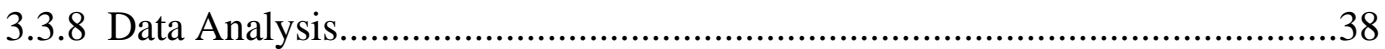

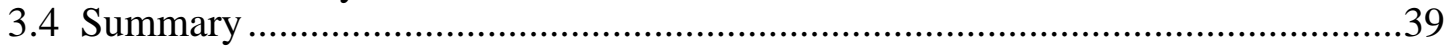

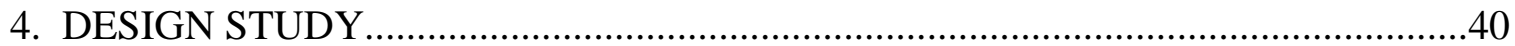

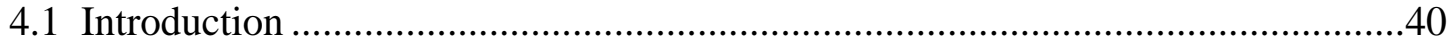

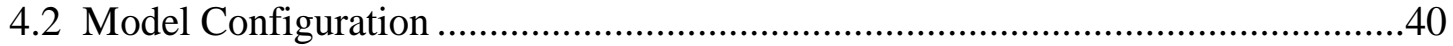

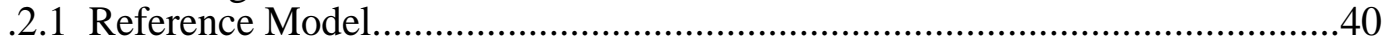

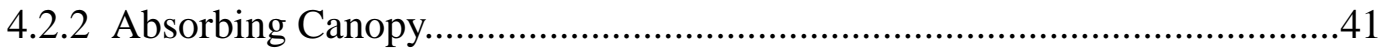

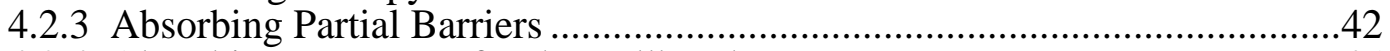

4.2.4 Absorbing Treatment for the Tollbooths .................................................45

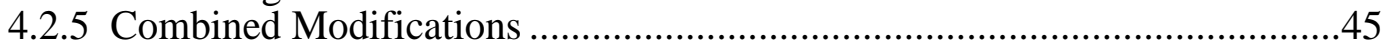

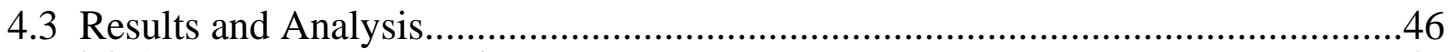

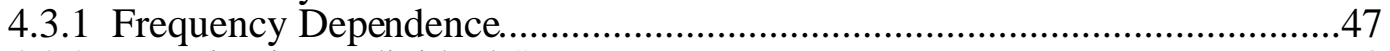

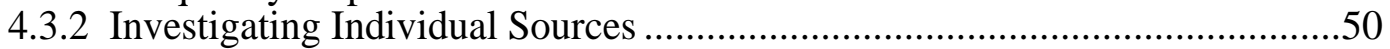

4.3.3 Performance of Passive Techniques.........................................................54

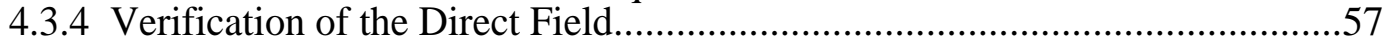

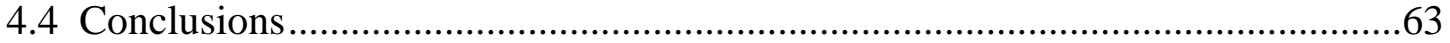

5. ACTIVE NOSIE CONTROL SYSTEMS ................................................................65

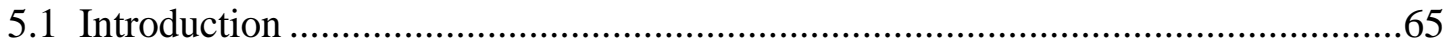

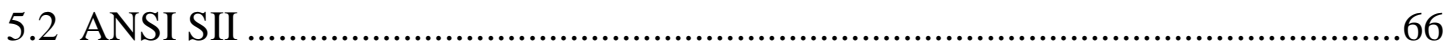

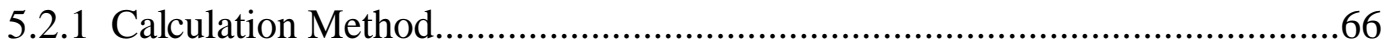

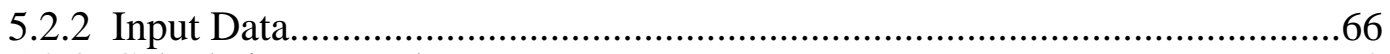

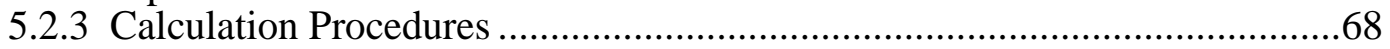

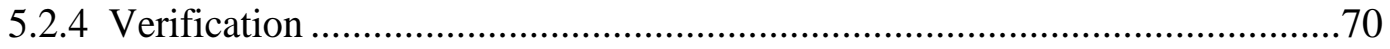

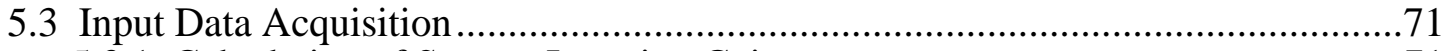

5.3.1 Calculation of System Insertion Gain .................................................... 71

5.3.1.1 Insertion Gain Test Signals........................................................74

5.3.1.2 Processing Recorded Time Domain Data ........................................75

5.3.2 Calculation of the Equivalent Speech Spectrum Level....................................77

5.3.3 Calculation of the Equivalent Noise Spectrum Level.....................................77

5.3.4 Calculation of the Equivalent Hearing Threshold Spectrum Level..................78

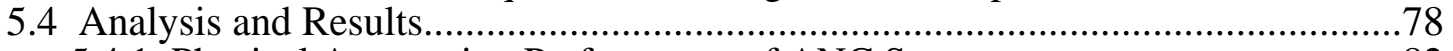

5.4.1 Physical Attenuation Performance of ANC Systems ..................................82

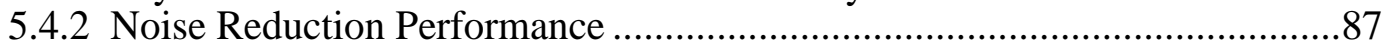

5.4.3 Speech Intelligibility Performance of ANC Systems ...................................88

5.4.3.1 Speech Intelligibility Performance for Typical Semi-Truck Noise ........89

5.4.3.2 Speech Intelligibility Performance for Idle Semi-Truck Noise.............96

5.4.3.3 Speech Intelligibility Performance for Idle Car Noise.......................100

5.4.4 Characteristics of Systems for Improved Communication ..........................104

5.4.4.1 Concepts for Improved Communication ........................................104

5.4.4.2 Sealed Headset with Directional Microphone ...................................105

5.4.4.3 Filtering the Directional Microphone Signal ...................................106

5.4.4.4 Directional Microphone Directivity Index.......................................110

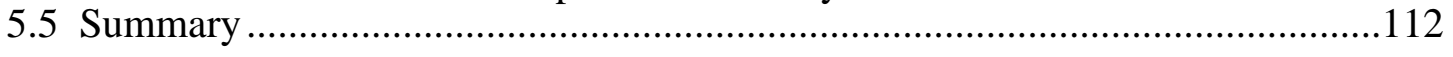

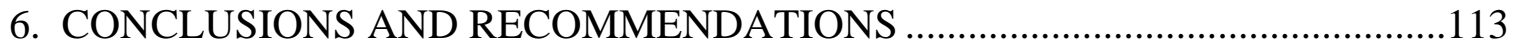

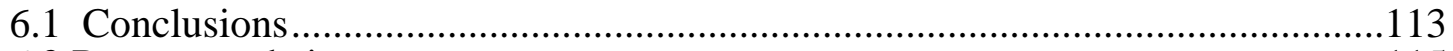

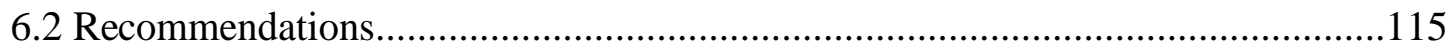




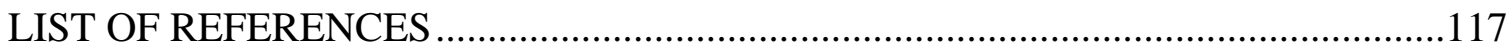

\section{APPENDICES}

Appendix A: Toll Road Employee Surveys...........................................................120

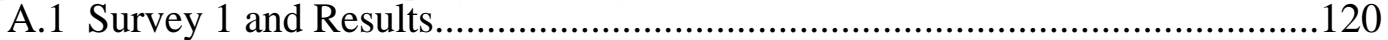

A.2 Survey 2 and Results.......................................................................126

Appendix B: Toll Plaza Beam Tracing Model......................................................130

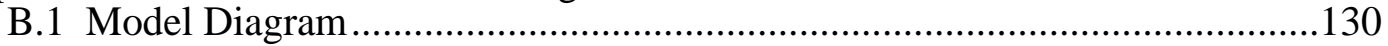

B.2 Frequency Dependence of Passive Noise Control Solutions..........................132

B.3 Overall Level per Source Pair ...........................................................................148

B.4 Direct Field Study, Semi-Truck Sources ......................................................197

B.5 Direct Field Study, Car Sources ........................................................ 210 


\section{LIST OF TABLES}

Table Page

3.1: Material properties used to model elements of the Portage Barrier Toll Plaza.........32

3.2: The semi-truck source spectrum, sound power level.........................................34

4.1: $\quad$ Table of the material characteristics assigned for the partial barriers with sound

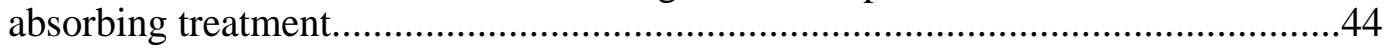

4.2: $\quad$ Material characteristics assigned for sound absorbing tollbooth walls..................45

4.3: The automobile source spectrum used in the direct field verification model...........61

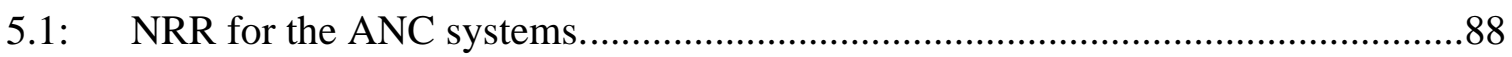

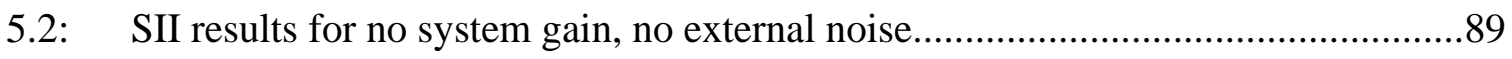

5.3: $\quad$ SII results for no system gain, actual traffic noise .........................................90

5.4: $\quad$ SII results in the presence of actual semi-truck noise .......................................95

5.5: $\quad$ SII results in the presence of actual idle semi-truck noise ..................................97

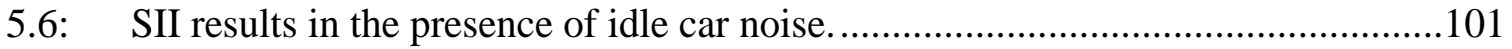

5.7: $\quad$ SII results for improved systems in the presence of idle semi-truck noise............109

5.8: $\quad$ SII results for improved systems in the presence of idle semi-truck noise............111 


\section{LIST OF FIGURES}

Figure Page

2.1: Photo of the north end of the Portage Barrier Toll Collection Plaza. View is facing

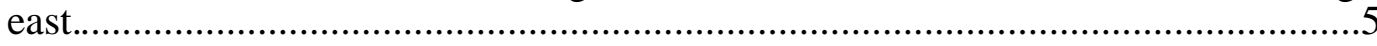

2.2: $\quad$ Evaluation of working conditions by tollbooth attendants..................................6

2.3: Rating of the annoyance of vehicle sound sources. Ratings were averaged among all respondents. A rating of 5.0 indicates that the respondents Strongly Agree that the noise emission of the particular vehicle is annoying, a rating of 3.0 indicates that the respondents are Undecided, and a rating of 1.0 indicates that the respondents Strongly Disagree that the noise emission of the particular vehicle is annoying. ......7

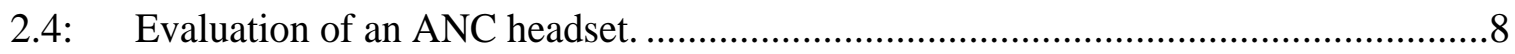

2.5: Block diagram of the single channel feedback ANC system. Representation modeled after Nelson \& Elliot $(1992$, p. 211) ....................................................... 12

2.6: Photo of error microphone and secondary source on an exposed ANC headset.....14

2.7: A single source and two geometric boundaries as modeled by a Mirror Image Source Method implementation. Open circle is the true source; closed circles are the image sources.

2.8: Diagram showing discrete diffraction paths over the top edge of a barrier. Open circle is the source; shaded circle is the receiver.

3.1: Case for a simple source over a perfectly reflecting plane. Schematic of the mesh and the receiver plane. The source was located at the coordinates $(50,50,0.5)$ in meters, and the receiver plane was located at the $\mathrm{Y}$-axis position of 50 meters.

3.2: Comparison between the beam tracing predictions and the analytical solution for a simple source over a perfectly reflecting plane. The difference in sound pressure level in $\mathrm{dB}$ over the receiver plane is displayed.

3.3: Diffraction by a planar rigid barrier over a reflecting plane. Schematic of the problem geometry, side view. The source is located at coordinates $(0,-7.5,0.5)$. The barrier is located at $Y=0$. The receiver is located at coordinates $(0,30,3)$. All positions are in units of meters. 
Figure

Page

3.4: Comparison between results obtained using a beam tracing simulation and the $\mathrm{K} \&$ A equation from Muradali and Fyfe (1997). The solid line represents data obtained using Lam's method and the dashed line represents data obtained using RAYNOISE

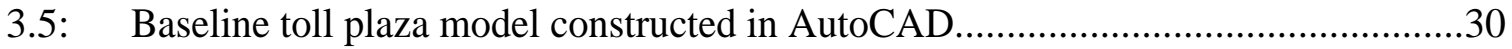

3.6: Location of semi-truck sound sources within the basic model of the toll plaza, top view.

3.7: Location of receiver points within the baseline model of the toll plaza.

4.1: Model of the Portage Barrier Toll Plaza used as the reference condition.

4.2: Toll plaza model with the canopy removed to simulate a perfectly absorbing canopy.

4.3: Toll plaza model with sound absorbing partial barriers

4.4: Toll plaza model with perfectly absorbing canopy, sound absorbing partial barriers, and absorbing treatment on the tollbooth walls.

4.5: $\quad$ Sound pressure level of all sources active as a function of frequency at the fourth window location to the north of the plaza. $\nabla$ level of existing toll plaza; * level with absorbing canopy; O level with absorbing barriers; level with absorbing barriers;

+ level with all modifications.

4.6: Insertion loss of all sources active as a function of frequency at the fourth window location to the North of the plaza. * insertion loss of absorbing canopy; O insertion loss of absorbing barriers; insertion loss of absorbing barriers; + insertion loss of all modifications....

4.7: Overall sound pressure level for an individual source pair as a function of window location. Active source pair is the middle idle semi-truck located in fourth lane from the north. $\nabla$ level of existing toll plaza; * level with absorbing canopy; O level with absorbing barriers; level with absorbing barriers; + level is all modifications......53

4.8: Insertion loss for an individual source pair as a function of window location. Active source pair is the middle idle semi-truck located in fourth lane from the north. * insertion loss of absorbing canopy; $\mathrm{O}$ insertion loss of absorbing barriers; insertion loss of absorbing barriers; + insertion loss of all modifications.

4.9: Overall level of all sources active as a function of window position. $\nabla$ level of existing toll plaza; * level with absorbing canopy; O level with absorbing barriers; level with absorbing barriers; + level is all modifications. 
4.10: Insertion loss of all sources active as a function of window position. * insertion loss of absorbing canopy; O insertion loss of absorbing barriers; insertion loss of absorbing barriers; + insertion loss of all modifications.

4.11: Overall level for all sources in the fourth lane from the north, semi-truck sources. $\nabla$ level of existing toll plaza; * level with of direct field validation model.

4.12: Insertion loss for all sources in the fourth lane from the north, semi-truck sources.

$\nabla$ total absorbing elements as specified in the direct field validation model. ...........60

4.13: Overall level for all sources in the fourth lane from the north, car sources. $\nabla$ level of existing toll plaza; * level with of direct field validation model.....

4.14: Insertion loss for all sources in the fourth lane from the north, car sources. $\nabla$ total absorbing elements as specified in the direct field validation model.

5.1: Photograph of the test configuration to obtain ANC system transfer functions......73

5.2: Location of the TechnoFirst ANC system with respect to the head and torso simulator.

5.3: $\quad$ Frequency response of the one-third octave band filters. ..................................76

5.4: $\quad$ Photo of the NCT Noise Buster Extreme ANC Headsets.....................................79

5.5: $\quad$ Photo of the NCT ProActive 1500 ANC Headsets.............................................. 80

5.6: $\quad$ Photo of the NCT ProActive 3500 ANC Headsets. ............................................81

5.7: Photo of the TechnoFirst Prototype External ANC System and the head and torso simulator.

5.8: Transfer function for the NCT Noise Buster Extreme ANC headset, right ear with the active noise control setting at the maximum position in terms of one-third octave bands for different signals. - random noise signal; .. speech signal; -- traffic signal.

5.9: Transfer function for the NCT ProActive 1500 ANC headset, right ear in terms of one-third octave bands for different signals. - random noise signal; .. speech signal; -- traffic signal.

5.10: Transfer function for the NCT ProActive 3500 ANC headset, right ear in terms of one-third octave bands for different signals. - random noise signal; .. speech signal; -- traffic signal. 
Figure

Page

5.11: Transfer function for the TechnoFirst external ANC system, right ear in terms of one-third octave bands for different signals. - random noise signal; .. speech signal; -- traffic signal.

5.12: Comparison of semi-truck traffic spectrum level density with speech spectrum level density for a distance of 1.0 meters from talker's lips to listener's ears. X normal speech; raised speech; $\nabla$ loud speech; + shout speech; O semi-truck traffic noise.

5.13: Comparison of semi-truck traffic spectrum level density with speech spectrum level density for a distance of 0.75 meters from talker's lips to listener's ears. X normal speech; raised speech; $\nabla$ loud speech; + shout speech; O semi-truck traffic noise.

5.14: Comparison of semi-truck traffic spectrum level density with speech spectrum level density for a distance of 0.5 meters from talker's lips to listener's ears. X normal speech; raised speech; $\nabla$ loud speech; + shout speech; O semi-truck traffic noise.

5.15: Comparison of idling semi-truck traffic spectrum level density with speech spectrum level density for a distance of 1.0 meters from talker's lips to listener's ears. X normal speech; raised speech; $\nabla$ loud speech; + shout speech; O idling semitruck traffic noise.

5.16: Comparison of idling semi-truck traffic spectrum level density with speech spectrum level density for a distance of 0.75 meters from talker's lips to listener's ears. X normal speech; raised speech; $\nabla$ loud speech; + shout speech; O idling semitruck traffic noise.

5.17: Comparison of idling semi-truck traffic spectrum level density with speech spectrum level density for a distance of 0.5 meters from talker's lips to listener's ears. $\mathrm{X}$ normal speech; raised speech; $\nabla$ loud speech; + shout speech; O idling semitruck traffic noise.

5.18: Comparison of idling car traffic spectral density with speech spectral density for a distance of 1.0 meters from talker's lips to listener's ears. X normal speech; raised speech; $\nabla$ loud speech; + shout speech; O idling car traffic noise.

5.19: Comparison of idling car traffic spectrum level density with speech spectrum level density for a distance of 0.75 meters from talker's lips to listener's ears. X normal speech; raised speech; $\nabla$ loud speech; + shout speech; O idling car traffic noise. 
Figure

5.20: Comparison of idling car traffic spectrum level density with speech spectrum level density for a distance of 0.5 meters from talker's lips to listener's ears. X normal speech; raised speech; $\nabla$ loud speech; +shout speech; O idling car traffic noise.

5.21: Masking profile due to a pure tone of $400 \mathrm{~Hz}$ at $80 \mathrm{~dB}$. Data from Egan and Hake

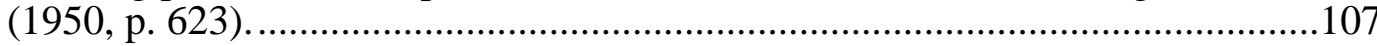


NOMENCLAUTURE

\section{Symbols}

$\begin{array}{ll}A_{i} & \text { band audibility } \\ \text { AI } & \text { articulation index } \\ \text { ANC } & \text { active noise control } \\ B_{i} & \text { larger of } N_{i}^{\prime} \text { or } V_{i} \\ \text { BEM } & \text { boundary element method } \\ C_{i} & \text { upward slope of speech masking } \\ C(s) & \text { transfer function between error microphone and secondary source } \\ \text { CFR } & \text { code of federal regulations } \\ d & \text { distance from talkers lips to speakers ears } \\ d_{0} & \text { reference distance } \\ D_{i} & \text { equivalent disturbance spectrum level } \\ D(s) & \text { signal of the primary source measured at the error microphone } \\ F_{i} & \text { insertion gain } \\ F_{i} & \text { igital audio tape } \\ E_{i} & \text { speech spectrum level } \\ E_{i} & \text { equivalent speech spectrum level } \\ & \end{array}$




$\begin{array}{ll}I_{i} & \text { band importance function } \\ L_{i} & \text { level distortion factor } \\ N_{i} & \text { noise spectrum level } \\ N_{i}^{\prime} & \text { equivalent noise spectrum level } \\ \text { NRR } & \text { noise reduction rating } \\ S & \text { speech intelligibility index } \\ \text { SII } & \text { speech intelligibility index } \\ \text { SIL } & \text { speech interference level } \\ \text { STI } & \text { speech transmission index } \\ T_{i}^{\prime} & \text { equivalent hearing threshold spectrum level } \\ \text { TNM } & \text { traffic noise model } \\ U_{i} & \text { standard speech spectrum level for normal vocal effort } \\ V_{i} & \text { self-speech masking spectrum level } \\ V_{i n}(s) & \text { voltage input of the secondary source } \\ V_{\text {out }}(s) & \text { voltage output of the error microphone } \\ X_{i} & \text { internal noise spectrum level } \\ X_{i}^{\prime} & \text { equivalent internal noise spectrum level } \\ Z_{i} & \text { equivalent masking spectrum level }\end{array}$




\section{IMPLEMENTATION REPORT}

Sealed ANC headsets with directional microphone input produced a significant increase in speech intelligibility. Subsequent investigations into modified ANC headsets that improve the signal to noise ratio and thereby the speech intelligibility are suggested. Advanced concepts should integrate adaptive algorithms to achieve the project goals. Feedforward algorithms are adaptive and therefore behave differently for different input signals. As a starting point, an adaptive algorithm may be placed between the directional microphone and the sealed ANC headset configuration. If implemented properly, the filter could provide significant improvements in speech intelligibility by selectively attenuate traffic noise and preserving speech at a level that does not overload the ear. Alternatively, the single channel feedback algorithm of existing ANC control headsets could be replaced by an adaptive ANC algorithm to yield similar results. Testing of various embodiments with respect to comfort should be performed. Investigation into cost and reliability should be addressed.

The toll plaza model demonstrated that passive noise control solutions which address the reflected sound field, do not substantially reduce the noise level. The modeling approach did not account for coherent reflections that cause reverberation and resonance. Due to the statistically random nature of vehicle noise, it is a reasonable approximation to use a source model assuming incoherent waves where phase is neglected. If resonance conditions occur at the toll plaza, placement of absorbing material may have a more dramatic effect than predicted by the model. It is suggested that the possible existence of resonance 
conditions be investigated in a subsequent study. On-site measurements at many locations throughout the toll plaza could be used to investigate the presence of standing waves. The results of the beam tracing method could be validated through on-site measurements. 


\section{INTRODUCTION}

Vehicular toll roads are one component of many municipal transportation systems. Toll collection facilities often include automated lanes and lanes with personnel working inside a booth. The automated lanes serve customers with exact change or electronic meters while the manual lanes serve customers with commercial vehicles and customers needing change or directions.

Personnel working at toll collection plazas are exposed to extended, continuous traffic noise. Previous studies of noise exposure levels for attendants working in Indiana toll collection plazas indicated that the equivalent A-weighted sound pressure levels experienced at the toll collection booths range between 52.5 to $84.2 \mathrm{~dB}$ (Arnold, 1998a, 1998b). While these values are below Occupational Safety and Health Administration (OSHA) regulations for engineering and administrative controls, they suggest that in certain cases a hearing conservation program may be required (National Archives and Records Administration, 2001a). In addition, exposure to a duration greater than eight hours, as in overtime labor or extended work shifts, may require further action.

Sustained noise levels of this nature may cause hearing loss, induce fatigue or stress, and reduce worker productivity. The annoyance and discomfort related to continuous noise exposure may create an unpleasant working condition and may affect the hospitality of the tollbooth operators and their attitude toward customers. Furthermore, the noise level may hinder communication with customers and may compromise safety. Reduction of the noise level and an improvement in speech intelligibility are highly desirable. 
Toll collection booths have certain features which are necessary for the business of toll collections but allow the transmission of noise. Open communication windows, often used in tollbooths, facilitate essential communication and monetary transactions. Typical transactions between toll collectors and patrons involve charge cards, commuter cards, toll tickets, and the exchange of cash. Direct visual and physical contact between patrons and attendants is made possible by the open communication window. One disadvantage is that vehicle noise generated outside the booth is easily transmitted into the booth via the open window. The booth structure acts as a partially open enclosure which may amplify road noise due to sound reflections off of rigid surfaces and reverberation within the enclosure.

In this investigation, the reduction of noise level and improvement of speech intelligibility at the location of the tollbooth attendant were studied. The thesis in organized as follows. Chapter 2 is a summary of the background material and preliminary investigation work. The beam tracing implementation used to model the sound field of the Portage Barrier Toll Plaza is presented in Chapter 3. Chapter 4 contains a discussion of different toll plaza configurations and the results of the beam tracing sound field analysis for each configuration. In Chapter 5, the speech intelligibility characteristics of active noise control systems are discussed. Conclusions and recommendations are presented in Chapter 6. 


\section{BACKGROUND}

Noise levels in many different industrial settings are high enough to be a health

concern and to interfere with communication. Industrial environments with structures and obstacles that cause sound to reflect, have sound fields that are similar to that within partially open enclosures. Though the primary application of interest for this research study involves tollbooths, the fundamental results are relevant to a general class of problems with similar characteristics. In this chapter, a summary of the background work and details of the literature review are included to place the subsequent work in the appropriate context.

\subsection{Toll Collection Plaza Description}

A typical toll collections facility, the Portage Barrier Toll Collection Plaza, was selected as the test location due to its high traffic volume and high noise levels. The toll plaza is located at milepost 24 on US toll road Interstate-90 which is west of Portage, Indiana. Interstate-90 facilitates traffic movement in east and west directions. Detailed information can be found in the blue prints of the facility (Indiana Toll Road, 1982). A photograph of the toll plaza is shown in Figure 2.1.

The toll plaza contains eleven tollbooths of two different sizes serving, at times, 12 lanes of traffic. The smaller tollbooths measure 3.05 meters long $(10 \mathrm{ft})$ by 1.52 meters wide $(5 \mathrm{ft})$ by 2.44 meters high $(8 \mathrm{ft})$ and are located on the extremes of the plaza to serve traffic movement in one direction. The larger tollbooths measure 4.57 meters long (15ft) 
by 1.52 meters wide $(5 \mathrm{ft})$ by 2.44 meters high $(8 \mathrm{ft})$ and are located at the center of the plaza to serve traffic movement in either direction. The structure of the booths is made of aluminum and stainless steel. Each booth has two doorway openings. The main door is open to assist patrons of the toll road. The secondary door is near the back of the booth; it is open when climatic conditions require. For the larger booths, the secondary door may also be used for toll collection. The booths are set upon raised concrete curb footings. In line with the booths, concrete crash impact barriers have been positioned in front of and behind the booth. The lanes are surfaced in concrete. Approximately 4.88 meters (16 ft) above the ground is an overhead canopy made of a tubular metal framework with corrugated metal sheets. The canopy has three main sections. The north and south ends of the canopy are characterized by a high and steep angle opening towards oncoming traffic while the center section is in a symmetric "A" configuration. Supporting the framework of the canopy are twelve large concrete columns located between a few of the booths and the respective crash impact barriers.

A preliminary inspection revealed that the booths were well built and did not appear to substantially contribute to the noise. Sound transmission through the openings of the tollbooths seemed to be the main noise issue. 


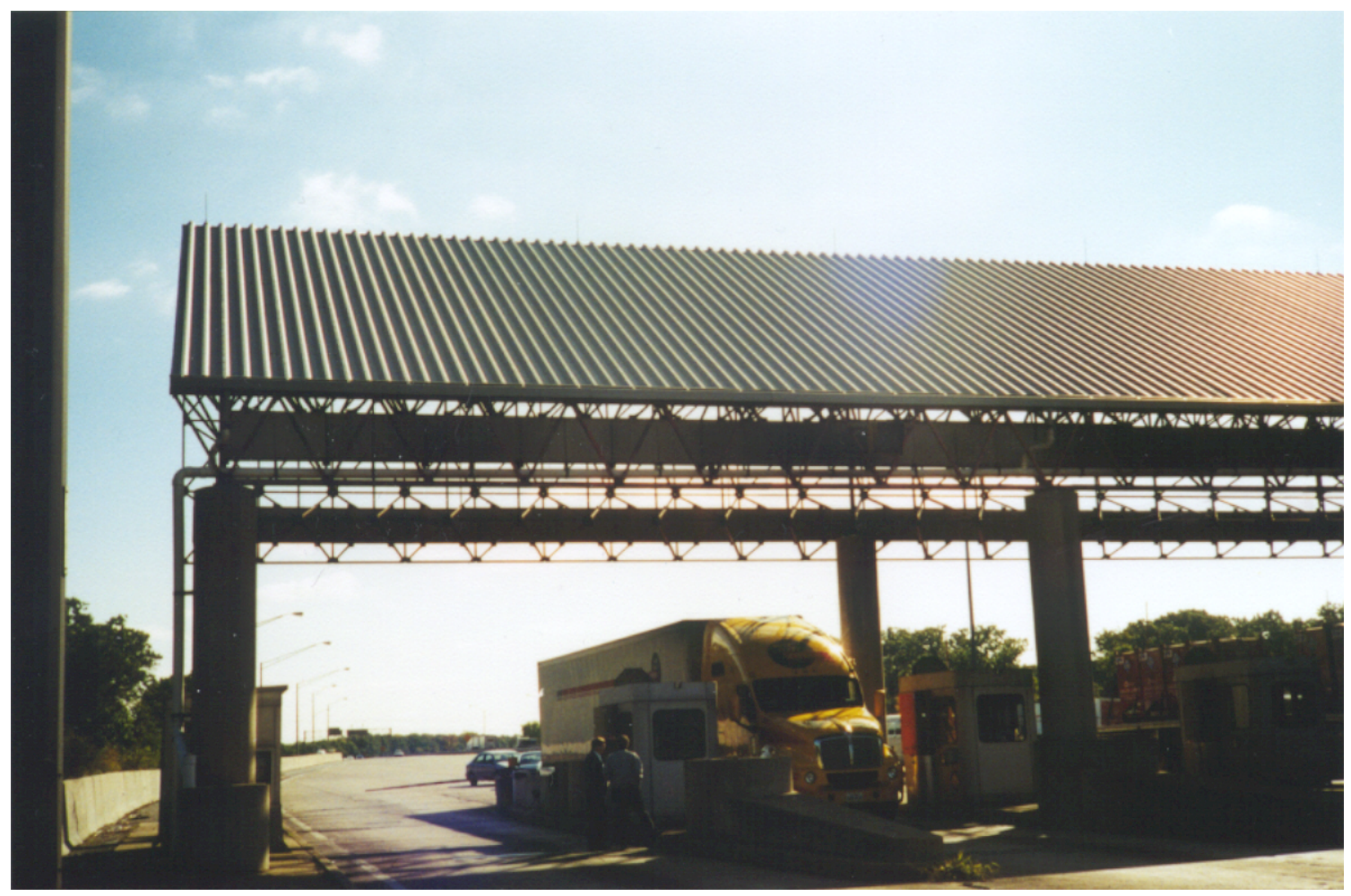

Figure 2.1: $\quad$ Photo of the north end of the Portage Barrier Toll Collection Plaza. View is facing east.

\subsection{Attendant Surveys}

Two surveys were administered to tollbooth employees working at the Portage Barrier Toll Collection Plaza. Questions were designed to be non biased and non leading. The surveys were administered in a blind and anonymous manner. Twenty nine toll plaza employees completed each survey. A copy of the surveys and a table showings the results are included in the Appendix. Further details of the surveys may be found in the article by Feist, Mongeau, and Bernhard (2001).

\subsubsection{First Attendant Survey}


The first survey was used to evaluate the employees' subjective response to the ambient noise levels in their occupational environment. A graphical display of the results is shown in Figure 2.2 and Figure 2.3. The results confirm that ambient noise is perceived as undesirable and impedes communication between operators and patrons. While the noise does not appear to induce physiological effects such as headaches, it may be responsible for temporary threshold shifts which are a preliminary indicator of hearing damage. In addition, the most annoying sources of noise were identified as semi-trucks and motorcycles during braking, acceleration, and idling. It is noteworthy that some of the most annoying sounds involve low frequency emissions.

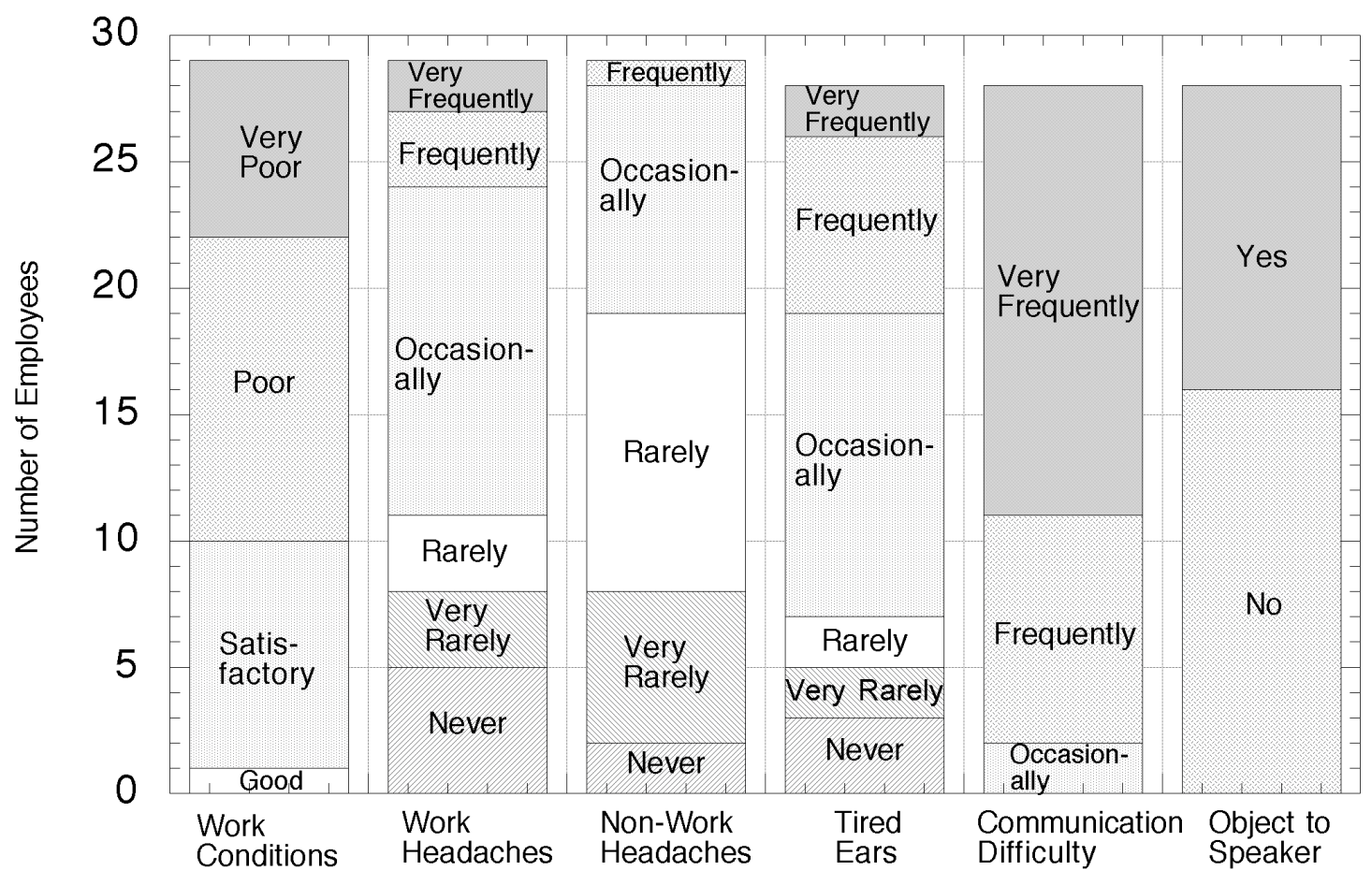

Figure 2.2: Evaluation of working conditions by tollbooth attendants. 


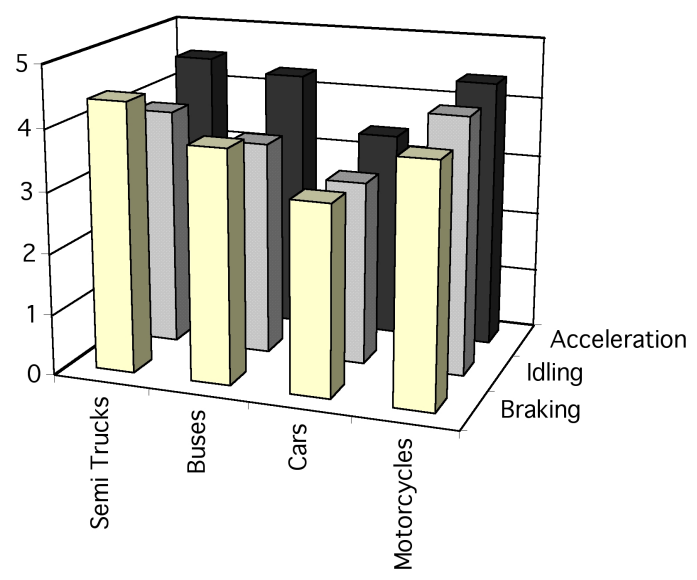

Figure 2.3: Rating of the annoyance of vehicle sound sources. Ratings were averaged among all respondents. A rating of 5.0 indicates that the respondents Strongly Agree that the noise emission of the particular vehicle is annoying, a rating of 3.0 indicates that the respondents are Undecided, and a rating of 1.0 indicates that the respondents Strongly Disagree that the noise emission of the particular vehicle is annoying.

\subsubsection{Second Attendant Survey}

In the second survey, tollbooth attendants were asked to evaluate a specific type of open ear active noise control (ANC) headphone and rate its performance with respect to noise reduction, speech intelligibility, and comfort. A graphical display of the results can be found in Figure 2.4. The results show that the active headset was effective at reducing ambient noise, but its ability to improve speech intelligibility could not be demonstrated. While the headset was thought to be uncomfortable by those surveyed, the results suggest that part of the discomfort was psychological and related to the appearance of the device. 


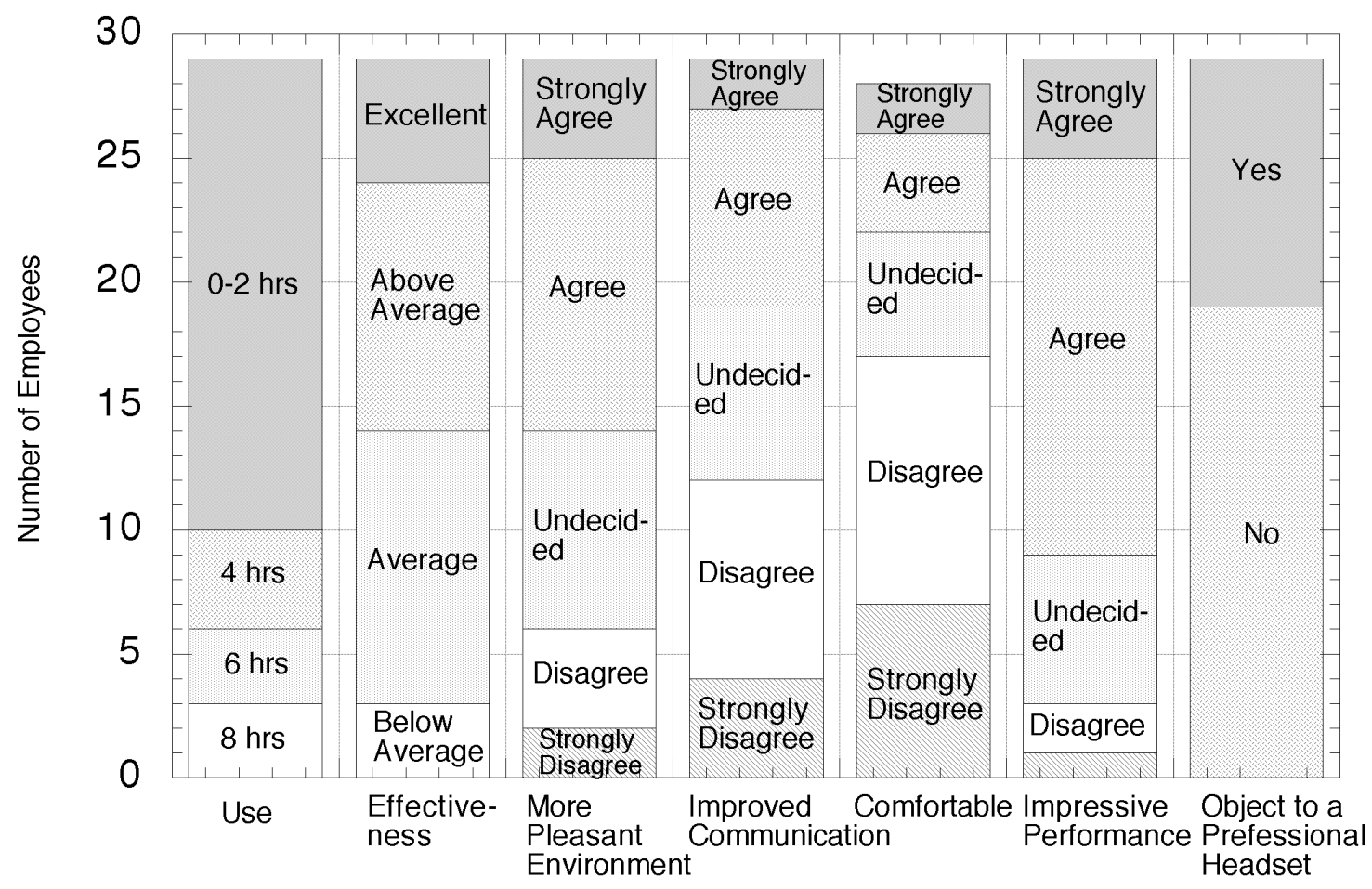

Figure 2.4: Evaluation of an ANC headset.

\subsubsection{Survey Conclusions}

The finding that environmental noise creates an unpleasant work environment and hinders communication substantiates the concerns of the project sponsors and justifies further investigation into potential noise control solutions for partially open enclosures. The toll plaza will be modeled to investigate the nature of the sound field and the performance of potential solutions.

Though the ANC headset did not clearly improve speech intelligibility, further investigation is reasonable due to the positive noise reduction performance. The type of ANC headset tested was a NCT Inc., Noise Buster Extreme. It is an open ear device built for listening to music through a portable entertainment system. Open ear headsets do not substantially suppress ambient noise through passive approaches such as circumaural cups. 
Given its consumer focus, the attenuation performance is not as strong as that of more industrial designs. Investigation of this and other ANC systems will focus upon noise attenuation and speech intelligibility performance.

\section{$\underline{2.3 \text { Potential Solutions }}$}

Potential noise control methods can be subdivided into two categories - passive techniques and active techniques. In this section, an overview of the current technology of each type of solution is presented. Once appropriate methods have been identified, the performance of each can be evaluated, though a discussion of the performance evaluation techniques is reserved for subsequent sections.

\subsubsection{Passive Noise Control Techniques}

Passive techniques for noise control can be based on mechanism of either reflection or absorption. A variety of noise control texts and handbooks explain the mechanism governing passive noise control and its effective application. A good introduction is presented by Fader (1981). The texts by Irwin and Graf (1979) and Bies and Hansen (1996) are suitable for a more advanced understanding. Practical information about performance and implementation can by found in the handbook by Faulkner (1976).

A sealed tollbooth where the attendant communicates by means of a speaker intercom system would alleviate many of the problems encountered. The sealed booth would block the transmission of sound by reflecting the sound away from the operator location. However, for reasons mentioned previously, this is not conducive to the business of toll collections. 
Creating barriers close to the vehicle locations that reflect the engine and exhaust noise away from the tollbooth location is another implementation of passive noise control. The frequency and attenuation performance of the barrier, however, depends upon its size. Traffic noise has significant low frequency components. To adequately deal with the low frequency sounds, the barrier would need to be large which entails considerable expense and space. In addition, a barrier may increase the sound level by creating a near reflection. A sound absorption treatment may be applied to the surface of the barrier to control reflections. The frequency performance of the absorption material depends upon thickness. As a general rule, a sound absorption treatment must have a thickness at least 1/4 of the wavelength of the lowest frequency of interest. To attenuate a $100 \mathrm{~Hz}$ tone, the material should be nearly 1 meter thick.

Reasonable implementation of absorbing material on partial barriers and on the exterior and interior of the tollbooths may reduce the effects of reflections and booth resonance. However, control of low frequency sound may be difficult to achieve and the transmission of noise through the open communication window will still present a problem. Various reflecting and absorbing configurations will be tested through toll plaza modeling,. Of particular interest is treatment of the canopy with sound absorption material, placement of sound absorbing partial barriers, and treatment of the exterior of the tollbooths with sound absorbing material.

\subsubsection{Active Noise Control Techniques}

An alternative approach to control low frequency sounds is by means of active techniques. Many texts contain information on ANC. A good introduction is given by Snyder (2000). Particularly clear and thorough discussions are presented by Nelson \& Elliot $(1992,1997)$. The reduction achieved by ANC works on the principle of 
superposition. When two identical waves, where one is shifted by a phase angle of 180 degrees from the other, are combined, the result is sound cancellation. Expanding upon this, an active noise system is used to create a sound with the same amplitude but opposite phase of an undesirable sound. When the two sounds are superimposed, the net result is a reduction of the undesirable sound.

The single channel feedback approach is a promising ANC implementation for the toll booth application. This approach is relatively simple compared with other ANC implementations, it is commercially available, and quite robust in performance. In addition, the feedback approach is particularly effective at attenuating low frequencies where the passive techniques described in the previous section are usually weakest.

A single channel feedback ANC system consists of an error microphone, a secondary source (loudspeaker), and a controller. A diagram of the system is shown in Figure 2.5. The error microphone is positioned very close to and a fixed distance from the secondary source. Given this fixed distance, an electro/acoustical system transfer function can be measured between the secondary source voltage input and the voltage output of the microphone. This measurable transfer function is represented in terms of Laplace transforms as

$$
C(s)=V_{\text {out }}(s) / V_{\text {in }}(s) \text {. }
$$

Considering the entire system, the input to the error microphone is representative of a sound field that contains a superposition of the primary source (the sound that is to be canceled) and the secondary source. The Laplace transform of the primary source signal measured at the error microphone will be designated $D(s)$. The combined output of the microphone is sent to a controller. The output of the controller drives the secondary source. The measured transfer function of the controller in term of a Laplace transform will be designated $G(s)$. The controller operates to minimize the sound pressure at the error 
microphone. As depicted in the figure, the measured signal at the error microphone in terms of Laplace transforms is

$$
E(s)=D(s)+G(s) * C(s) * E(s) .
$$

which can be written as

$$
E(s)=D(s) * 1 /[1-G(s) * C(s)]
$$

The signal at the error microphone will be a minimum when the denominator is a maximum. Given a measured electro/acoustical system transfer function between the acoustical transducers, $C(s)$, the greatest attenuation is gained by selecting a controller transfer function $G(s)$ that maximizes $|G(s) * C(s)|$.

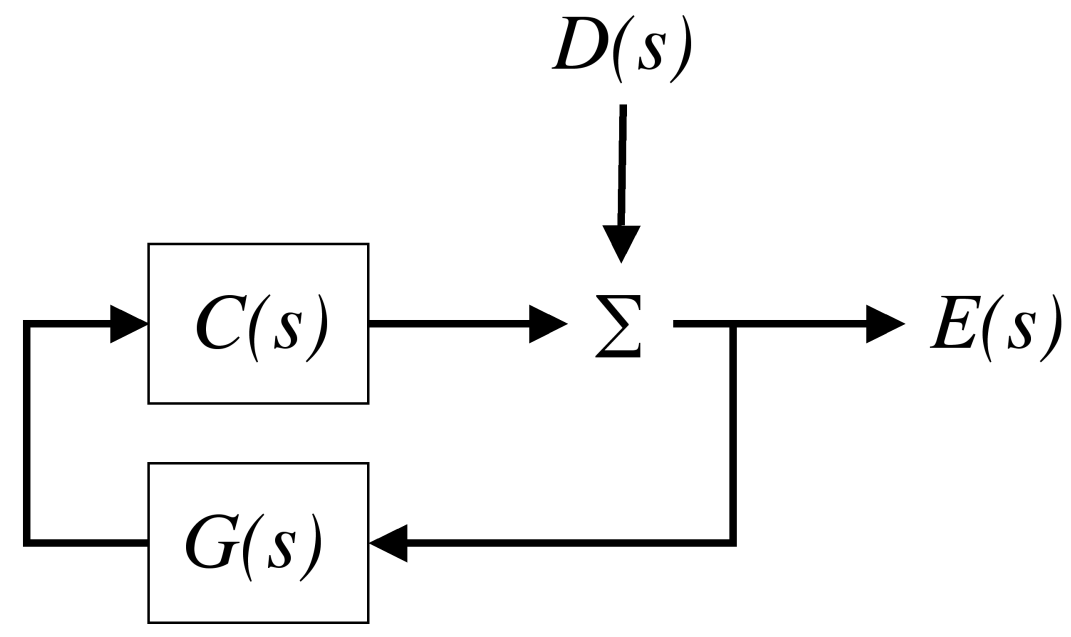

Figure 2.5: Block diagram of the single channel feedback ANC system. Representation modeled after Nelson \& Elliot (1992, p. 211).

In a physical sense, the controller operates by shifting the signal received at the error microphone by 180 degrees, amplifying, and sending the inverted signal to the secondary source and back to the error microphone. For ideal transducers, specifically transducers with a flat phase response, the system would have no time lag due to processing. Therefore, the high frequency limit of the ANC system would be determined by the delay due to the 
spacing between the error microphone and the secondary source. At a wavelength equal to twice the spacing, inverting and amplifying the signal will cause the gain at the error microphone to be unbounded. In application, systems have lag due to the finite speed of signal transfer, transducer inertia, and controller computational time. Therefore the first instability occurs at a frequency lower than predicted for the ideal system. In addition, actual transducers exhibit phase shifts and manufacturing inconsistencies which further alter the instability points. To compensate for such instabilities, the controller must have a gain less than one at the instability frequencies. While a digital controller may be used to correct for the transducer phase anomalies to gain attenuation over a greater frequency range, the additional complexity currently requires too much computational time and increases delay. For efficiency and simplicity, analog controllers are often designed to only pass frequencies below the first instability.

The potential of ANC systems to reduce low frequency sounds within reverberant, partially open enclosures has been demonstrated in previous studies by Waters (1988) and Waters and Bernhard (1989). The impact of such systems on speech intelligibility, however, is not well understood. Since low frequency sounds can mask speech signals, single channel feedback ANC system embodiments, which generally display a strong low frequency attenuation, may increase intelligibility.

The single channel ANC implementations of interest include: multiple headset configurations and an external configuration that can be mounted to the tollbooth. The acoustic volume of control is relatively small for ANC headsets since the acoustic pressure is minimized in the immediate vicinity of the ear. An exposed view of the ANC arrangement used in a headset is shown in Figure 2.6. The close positioning of the error microphone and the secondary source in the headset configuration allows a broader band to be actively attenuated. Therefore, the performance for ANC headsets is indicative of the best achievable reduction for any single channel feedback ANC solution. While the external ANC system 
will have lower ANC attenuation due to physical limitations, it does not contain passive components which may impede communication. Furthermore, the external configuration has none of the comfort concerns associated with headsets. Investigation of ANC systems will focus upon noise attenuation and speech intelligibility performance.

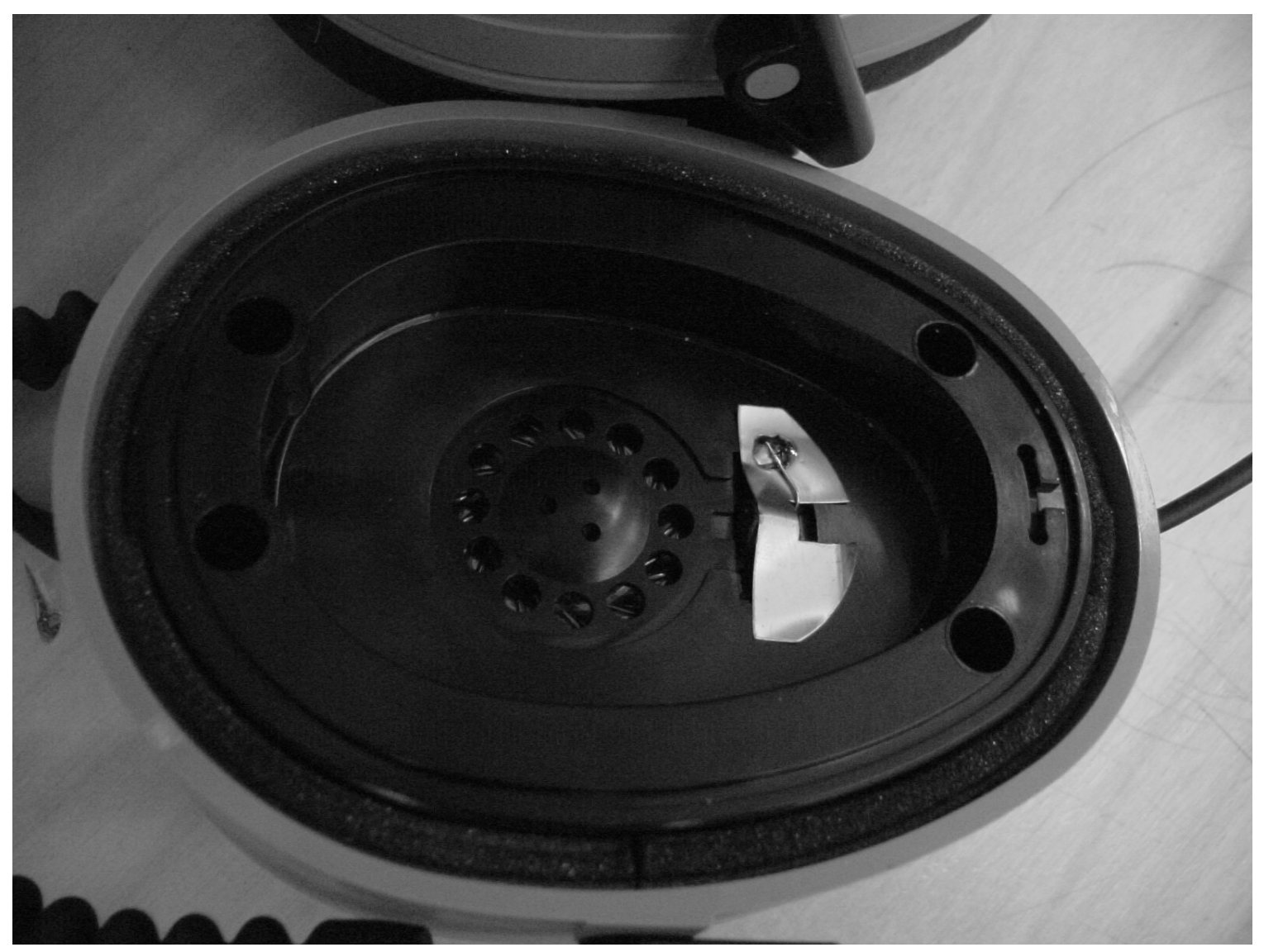

Figure 2.6: Photo of error microphone and secondary source on an exposed ANC headset.

\subsection{Modeling of the Toll Plaza}

After inspection of the toll plaza and analysis of the survey results, the optimal acoustical layout of the plaza was considered. Modeling of the toll plaza was proposed to investigate the sound field and explore the effects of noise control strategies. Two configurations were considered for the model: 1. a physical scale model and 2. a software 
based model. The reduced scale physical model limited test configuration in terms of materials and geometries. A software model flexible enough to allow many configurations was advantageous.

The standard commercially available acoustical software packages either utilize a boundary element method (BEM), such as LMS SYSNOISE and Automated Analysis Corporation COMET/Acoustics, or employ a beam tracing method such as LMS RAYNOISE. An addition option, FHWA's Traffic Noise Model (TNM) software package, was specifically designed for traffic noise predictions. TNM makes use of: 1. a simplified geometric approach to model sound propagation and diffraction and 2. a database of look up tables for sources. BEM packages can model sound radiation from structural surfaces as well as propagation through air. The beam tracing method can model sound propagation through air but cannot model sound radiation from structures. However, BEM programs entail a high level of complexity. In contract of a beam tracing technique, BEM programs require additional time necessary to construct the model, additional computational time, and the results would be at a level of complexity making it more difficult to ascertain the fundamental concepts at work. At the other level of approximation, the FHWA Traffic Noise Model is too simplistic and not flexible enough to model toll plaza noise control strategies. For this analysis, the beam tracing method used within LMS RAYNOISE is a good technique to evaluate the acoustics of the toll plaza. It contains the correct level of approximation to efficiently and accurately model the toll plaza. In addition, RAYNOISE is flexible enough to test the noise control strategies.

\subsubsection{The LMS RAYNOISE Software Package Theory}

Three numerical methods for steady state acoustical modeling of non-curved geometries are presented. The first two are common techniques. The third method is a 
hybrid of the previous two techniques and is the one implemented within LMS

RAYNOISE. None of these techniques account for diffraction in their original formulation. The implementation of diffraction edges is handled separately in LMS RAYNOISE and is discussed at the end of this section. For more information concerning the different numerical methods, refer to the RAYNOISE user's manual (LMS Numerical Technologies, 1998).

\subsubsection{Mirror Image Source Method}

The mirror image source method is a simple and accurate way to model sound source and barrier interactions. In Figure 2.7, a basic mirror image source method implementation is depicted. The mirror image source method treats a geometric bound as a mirror. An image source is positioned opposite of the source location as reflected by the boundary. The image source is used to account for sound reflection resulting from the boundary. Once the image source is located, the boundary is removed. A perfectly reflecting boundary is modeled by assigning the source and the image source the same amplitude and phase characteristics. Assigning a lower strength and a lagging phase to the image source models a loss of energy and a boundary with a complex impedance.

The mirror image source method can be extrapolated to multiple sources, multiple boundaries, and multiple orders of sound reflection. The major disadvantage of this technique is the high level of computation necessary to correctly locate all of the image sources and assign the correct characteristics to each image source. Due to the nature of the calculation, the computational time increases exponentially with reflection order (LMS Numerical Technologies, 1998, chap. 3.1). In addition, this technique does not model the nearfield of the source. For further information about the method of image sources, refer to Kuttruff (1997). 


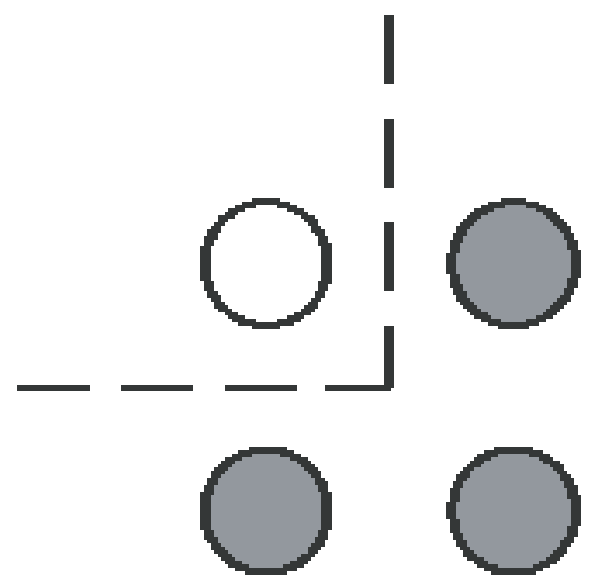

Figure 2.7: A single source and two geometric boundaries as modeled by a Mirror Image Source Method implementation. Open circle is the true source; closed circles are the image sources.

\subsubsection{Ray Tracing Method}

The ray acoustics method, also known as the geometrical acoustic method, models sound wave propagation as rays much like light waves behaves in optics. For the reader interested in the mathematical development of ray tracing, the text by Ziomek (1985) is a good place to start. Advanced and though discussions can be found by Lunenburg (1964) and Born (1964).

The ray tracing method is a more computationally efficient model of sound propagation than the mirror image source method. Necessary for a ray tracing model is the specification of a source, a receiver volume, and the any geometric boundaries. Reflection, transmission, and absorption characteristics describe the boundary. Radiation and strength characteristics are specified for the source. In the ray tracing method, the source energy is divided into a discrete number of rays with energy assigned to the rays in a manner consistent with the radiation directionality of the source. The rays travel outward from the source at the speed of sound; the ray strength is attenuated with distance due to spherical divergence and absorption of the medium. Upon contact with a boundary, part of the 
energy is absorbed, part of the energy is transmitted, and part of the energy is reflected in accordance with the properties assigned to the boundary. The transmitted ray has the same orientation as the original (unless the refraction of the boundary is specified) while the law of specular reflection indicates the direction of the reflected ray. The energy of reflected and transmitted rays are calculated from the assigned boundary characteristics. When the energy of the ray falls below a certain threshold, the ray is abandoned. Each ray is checked to see whether it crosses a receiver volume. Rays crossing the receiver volume give a measure of sound pressure level.

The major advantage of this technique is that computation time only increases linearly with reflection order (LMS Numerical Technologies, 1998, chap. 3.1). However, certain limitations are associated with this technique. The ray nature of sound waves and therefore the ray tracing method is only a good approximation for high frequencies. For accurate coherent source modeling, distance between the source and the receiver (which is also known as the characteristic length) should be less that the wavelength of sound emitted by the source, (Weston, 1997) and (Felsen, 1997).

\subsubsection{Beam Tracing Method}

The beam method is quite similar to the ray tracing method. As in the ray tracing method, the source emits a finite number of rays. In the beam tracing method, though, a ray describes the center axis of a beam of radiation. The beams touch each other and form a complete surface. The direction of beam propagation is determined by a ray tracing algorithm for the axis of the beam. The beams increase in cross-sectional area according to spherical divergence. When the beam crosses a receiver point, the location of the crossing is used to calculate the position of an image source. Accounting for losses due to medium 
attenuation, surface attenuation, and spherical divergence, the strength of this image source is calculated.

In essence, the beam tracing function is used as an efficient way to identify the position and strength of image sources. The image sources are used to calculate sound pressure level at the receiver points. The computational efficiency of the ray tracing method is combined with the accuracy of the mirror image source method which makes this a good choice for the toll plaza simulation problem. Even though this method is more accurate than the ray tracing method, limitations exist with this approach. The beam tracing method is most accurate when the wavelength is less than the characteristic distance. However, this limitation involves coherent source modeling. Since vehicle noise has a random characteristic when averaged over an extended length of time, the phase angle of sound pressure may be not important. Therefore, for this research, the incoherent source model is more appropriate than the coherent source model. By neglecting the phase angle of sound pressure, the energy is calculated without interference patterns. An additional limitation of the beam tracing method, a phenomena known as cone narrowing, can occurs when the beam intersects an interior corner boundary and the ray axis only accounts for one surface interface (LMS Numerical Technologies, 1998, chap. 3.1). The manual describes methods to statistically account for cone narrowing (LMS Numerical Technologies, 1998, chap. 3.1). However, the toll plaza model will contain exterior geometries which should limit the effects of cone narrowing.

\subsubsection{LMS RAYNOISE Diffraction Technique}

Diffraction involves the bending of sound waves passing over an obstacle. The Kurze-Anderson method used in RAYNOISE is a first order approximation utilizing the single shortest path between the source and the receiver diffracting over a barrier edge 
(LMS Numerical Technologies, 1998, chap. 3.3). In this way, a diffraction edge is characterized by a discrete point of diffraction along the edge. The attenuation due to the barrier is then the difference between the diffracted path length and the shortest path length without the barrier present. To model edge effects at the toll plaza, the diffraction technique used in RAYNOISE was deemed to be sufficient.

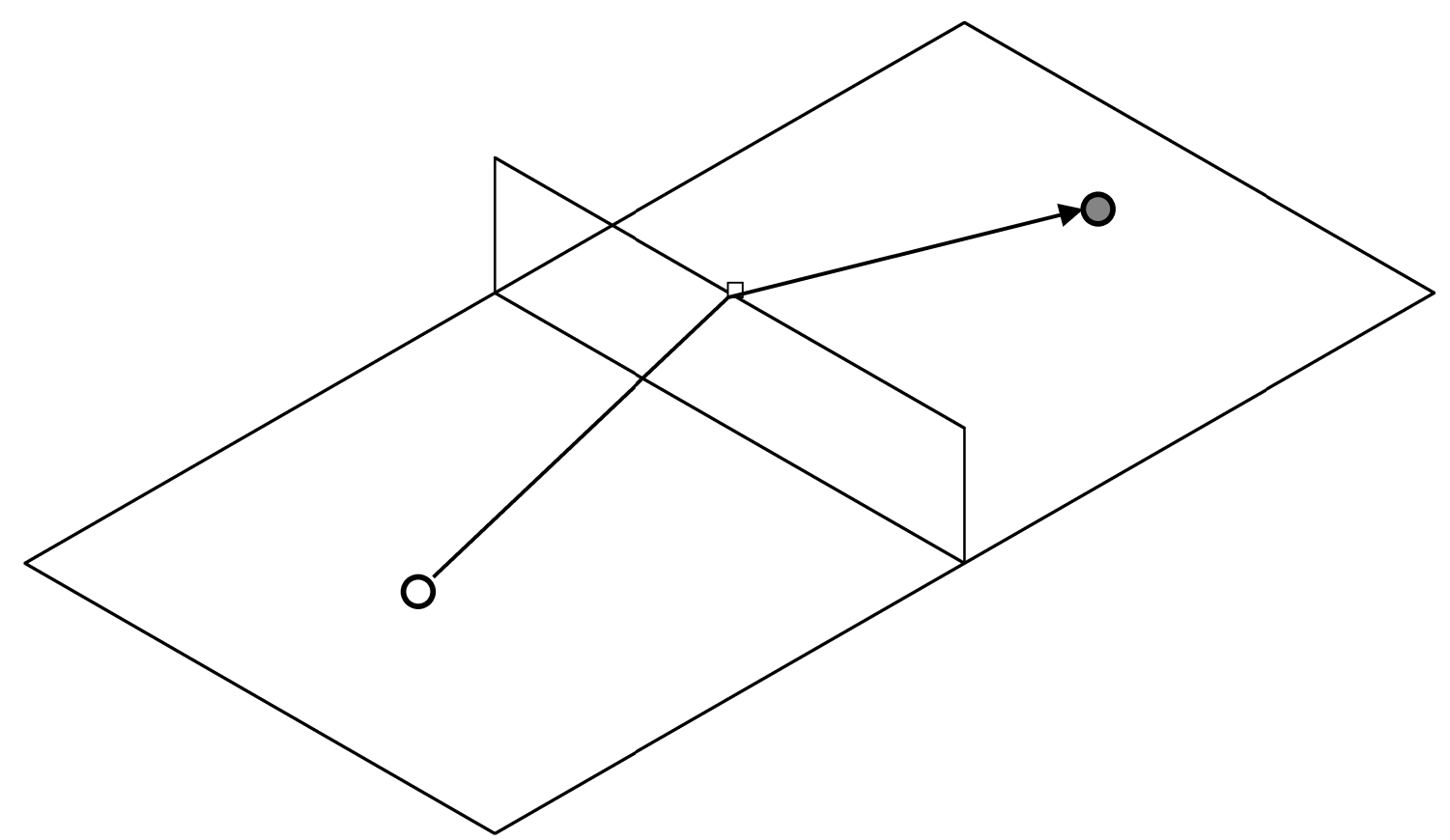

Figure 2.8: Diagram showing discrete diffraction paths over the top edge of a barrier. Open circle is the source; shaded circle is the receiver.

\section{$\underline{2.5 \text { Speech Intelligibility }}$}

Speech intelligibility involves the ability to understand speech communication in a particular environment or through a particular system. Calculation of speech intelligibility from physical parameters is the primary area interest for this research. The speech intelligibility of possible noise control solutions such as ANC headsets may be objectively measured through the use of a suitable method. 


\subsubsection{Standard Intelligibility Calculation Methods}

In this section, standard methods to calculate the intelligibility, articulation, or interference of speech will be summarized. The most suitable method for calculating the effects of the ANC systems on speech intelligibility will be identified. A brief overview of the methods may be found in Crocker (1997b) and Tocci (1997). The interested reader is referred to the compilation of papers reviewed by Hawley (1977) which contains a comprehensive citation of the historically prominent works in the field of speech intelligibility.

The Speech Interference Level (SIL), as specified by ANSI S3.14-1977, is a simple metric that estimates the degree to which a particular noise interferes with communication (American National Standards Institute, 1977). Given a distance between a talker and a listener and the SIL due to the background noise, the vocal effort for reliable communication can be predicted. The sound pressure level for noise in the four octave bands with center frequencies between $500 \mathrm{~Hz}$ to $4000 \mathrm{~Hz}$ are used for calculating the SIL. The metric is obtained by averaging the four octave bands on an energy basis and expressing the result as a sound pressure level.

The Articulation Index (AI), as specified by ANSI S3.5-1969, utilizes a weighted average signal to noise ratio (American National Standards Institute, 1969). While the AI metric is historically rich and has been a popular standard for quite some time, it has been replaced by the Speech Intelligibility Index (SII) which will be discussed in a following paragraph.

The Speech Transmission Index (STI) was proposed by Houtgast and Steeneken (1984) as a metric to account for speech distortion due to enclosure reverberation.

Fundamental to the metric is the calculation of a modulated transfer function which indicates 
the degree of distortion due to reverberation. The reverberant noise and the interfering noise are combined to develop an apparent noise level. Beyond the introduction of reverberant noise, many of the calculation procedures follow earlier versions of the AI using a signal to noise ratio technique.

The Speech Intelligibility Index (SII) is the most recently developed of the methods described and was standardized in ANSI S3.5-1997 (American National Standards Institute, 1998). Simply, the SII is a weighted average of the signal to noise ratio taking into account overloading and masking effects. The SII is specified for use with octave band, one third octave band, or critical band data. The minimum data necessary includes the following: the noise spectrum per specified calculation band, the listener's hearing threshold per specified calculation band, and the vocal effort of the talker. In addition, the SII can be used to account for a system transfer function between the talker and listener, the type of speech information, and the type of noise such as reverberant distortion.

For the purposes of evaluating the speech intelligibility of single channel feedback ANC systems, the SII is a good choice. The SII is appropriately sophisticated to achieve results highly correlated to actual communication situations. Since single channel feedback ANC systems are non-adaptive, the attenuation is linear. As long as an ANC system behaves linearly in the tested frequency bands, the effects of the ANC system can be accounted for simply from its transfer function. As well as speech intelligibility performance, the physical attenuation of each device for noise reduction purposes is also of interest. The system transfer functions may used to evaluate the noise attenuation performance of the ANC systems.

\section{$\underline{2.6 \text { Summary }}$}


Survey results indicate that noise at the toll plaza creates an unpleasant work environment and hinders communication. Reasonable passive noise control strategies will be modeled using a beam tracing method. To address improvements in speech intelligibility along with additional noise reduction, ANC systems will be explored. Various ANC headsets and a prototype ANC system designed to be mounted to the tollbooth and create a local region of attenuation will be evaluated. For the purposes of evaluating the speech intelligibility of the ANC systems, the SII as set forth by ANSI S3.5-1997 will be used. The system transfer functions will used to evaluate the noise attenuation performance of the ANC systems. 


\section{MODELING OF THE TOLL PLAZA}

In this chapter, the application of the LMS RAYNOISE software package to model the acoustics of the Portage Barrier Toll Plaza will be described.

\section{$\underline{3.1 \text { Introduction }}$}

Appropriate and feasible noise control solutions can be best identified from a good understanding of the sound field and nature of the sound transmission paths. A computer model of the physical features of the toll plaza is one possible approach to gain this understanding. Computer models also allow the performance of potential noise control solutions to be assessed. A beam tracing method was employed, using LMS RAYNOISE. This method was deemed appropriate and efficient for this study.

LMS RAYNOISE is well suited for the evaluation of noise control strategies that involve architectural changes. Strategies that will be discussed in Chapter 4 include the treatment of the existing overhead canopy with sound absorbing materials, the implementation of partial barriers with absorbing surface treatments, and the application of sound absorbing material to tollbooth surfaces. In this chapter, the fundamental elements of the computer model are described. The justification for key assumptions and for the selection of different adjustable parameters is discussed. 


\section{$\underline{3.2 \text { Verification }}$}

Before proceeding with the analysis of a toll plaza, two simple models were constructed to test the accuracy of the beam tracing technique employed within RAYNOISE. The models were compared with known analytic solutions.

\subsubsection{Verification Case 1}

Ray acoustic models may be inaccurate for low frequency sources where the ray approximation breaks down or for models with small characteristic distances - the distance from the source to the first reflecting surface or to the receiver (Crocker, 1997a and Weston, 1997). The first verification model was constructed to investigate these limitations.

Following standard modeling procedures, a 100 meters by 100 meters single element mesh (plane) was created within AutoCAD. The plane was imported into RAYNOISE. A $100 \mathrm{~Hz}$ coherent source was placed in the center of, and one meter above the plane. A receiver mesh with dimensions 98 meters by 100 meters centered one meter above the source was created. The sound pressure at node points of the receiver mesh was calculated in RAYNOISE. A diagram of the model is included in Figure 3.1.

Code was written to obtain an analytical solution for the same configuration. The theory and the analytic model are discussed by Kinsler and Frey (1982). An infinite reflecting plane was modeled using one mirror image source. The primary source and the image source were identical monopoles. The sound field was calculated at the same locations as those of the mesh points in the beam tracing model. The RAYNOISE sound pressure level data was imported into MATLAB and compared with the analytical solution as displayed in Figure 3.2. There is an excellent agreement between the beam tracing predictions and the analytic solution. Close to the source, there is a small discrepancy of $0.02 \mathrm{~dB}$ between the sound pressure levels which diminished as the characteristic distance 
increased. Low frequencies and small characteristic distances were not problematic for the beam tracing case considered in this study. The accuracy of the predictions for this case was adequate to simulate noise conditions at the toll plaza.

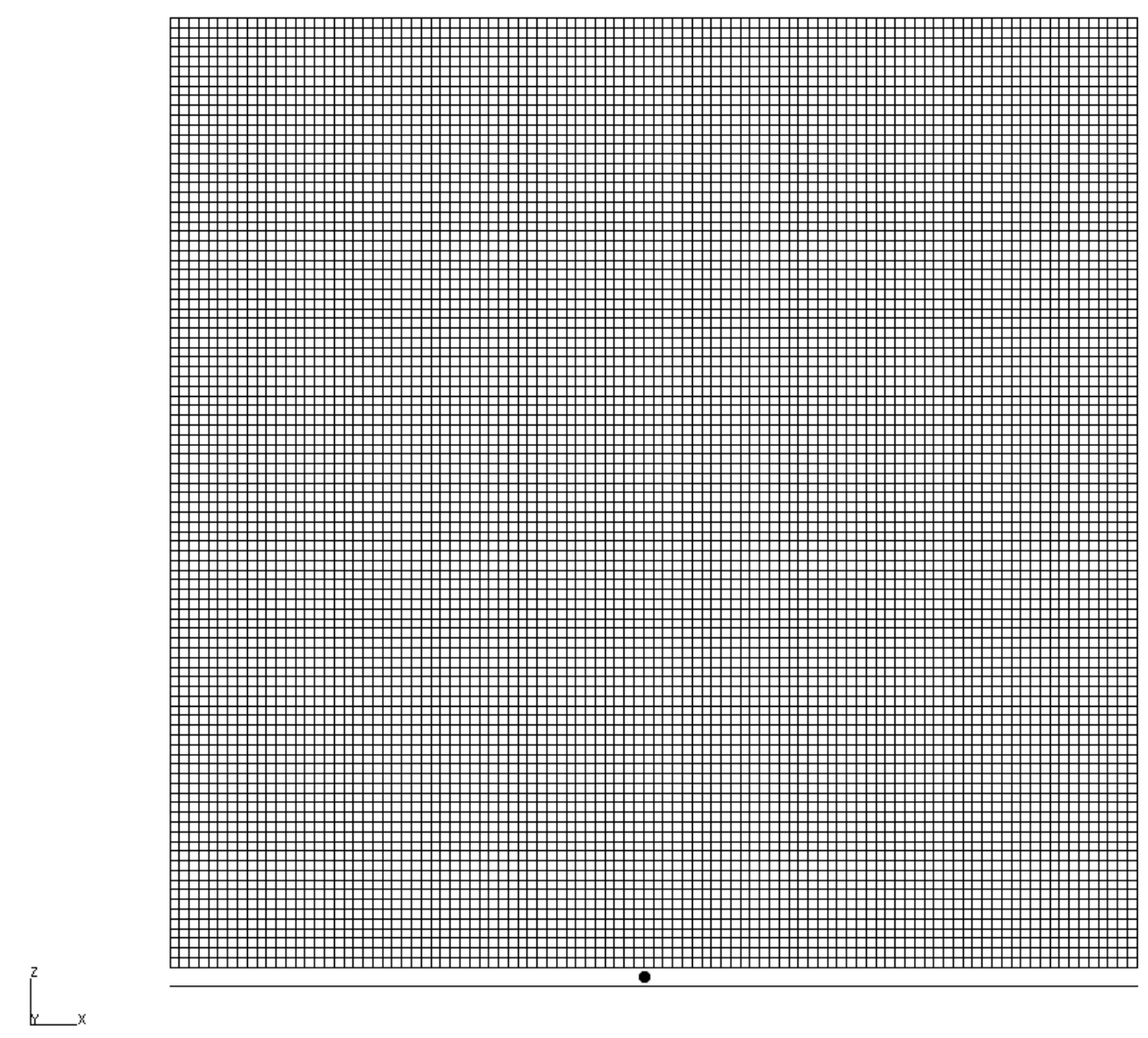

Figure 3.1: Case for a simple source over a perfectly reflecting plane. Schematic of the mesh and the receiver plane. The source was located at the coordinates (50, $50,0.5)$ in meters, and the receiver plane was located at the Y-axis position of 50 meters. 


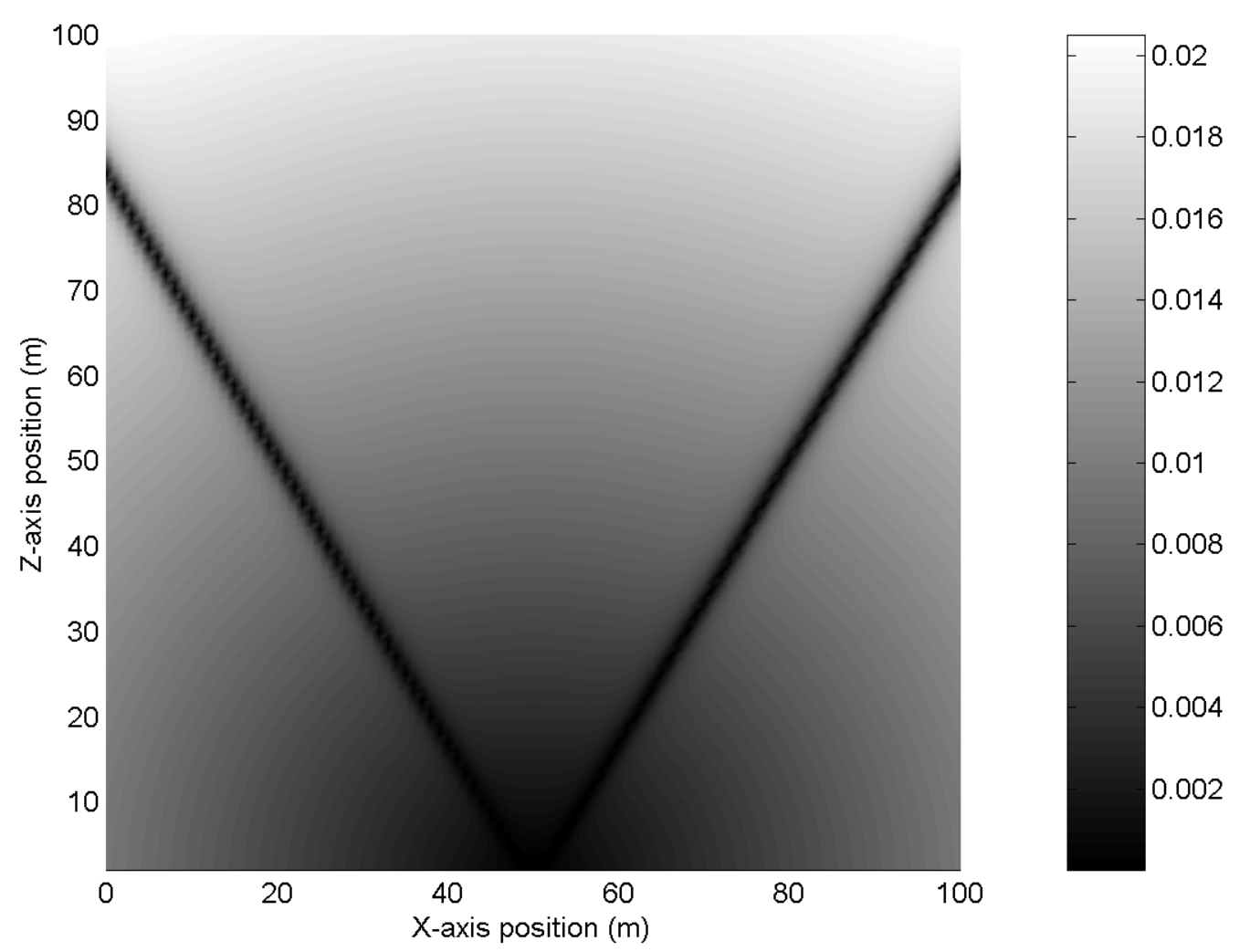

Figure 3.2: Comparison between the beam tracing predictions and the analytical solution for a simple source over a perfectly reflecting plane. The difference in sound pressure level in $\mathrm{dB}$ over the receiver plane is displayed.

\subsubsection{Verification Case 2}

Since a realistic model of a toll plaza involves diffraction of sound around the tollbooths, a second case was designed to investigate the edge diffraction technique used within RAYNOISE. Following standard modeling procedures, AutoCAD was used to create a plane of dimensions 200 meters by 200 meters with a barrier attached to and positioned in the center of the plane. The barrier width was 10 meters, and its height was 3 meters. The AutoCAD generated surfaces were imported into RAYNOISE. A point source was positioned in the center and perpendicular to the axis of the barrier, 0.5 meters above the ground plane and 7.5 meters in front of the barrier. An ideal receiver was positioned in 
the center and perpendicular to the axis of the barrier, 3 meters above the ground plane and 30 meters behind the barrier. The top and both side edges of the barrier were specified as diffraction edges, which means that the sound pressure in the shadow region of the obstacle was calculated along these edges. The analysis was performed for one-third octave bands center frequencies from $20 \mathrm{~Hz}$ to $20,000 \mathrm{~Hz}$. Figure 3.3 includes a diagram of the model for this verification case.

An approximate analytical solution for the same test situation was obtained based on Lam's method using Kurze and Anderson's equations as presented by Muradali and Fyfe (1997). Lam's method accounts for phase interactions, which is consistent with the coherent source approach used within RAYNOISE. The method assumed an infinite reflecting plane modeled using mirror image sources. All other parameters were the same as for the ray tracing simulation.

A comparison between the ray tracing model predictions and the analytical result is displayed in Figure 3.4. There is a good agreement between the RAYNOISE results and the analytic solution over the frequency range between $125 \mathrm{~Hz}$ to $1600 \mathrm{~Hz}$. There are discrepancies at higher frequencies. The discrepancy are curious since both methods approximate a diffraction edge using the single shortest diffraction path from point source to point receiver. The accuracy of the model was again was deemed satisfactory.

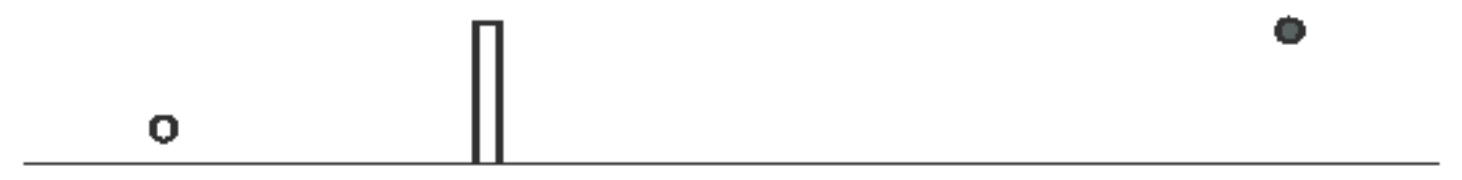

Figure 3.3: Diffraction by a planar rigid barrier over a reflecting plane. Schematic of the problem geometry, side view. The source is located at coordinates $(0,-7.5$, $0.5)$. The barrier is located at $Y=0$. The receiver is located at coordinates $(0$, 30,3). All positions are in units of meters. 


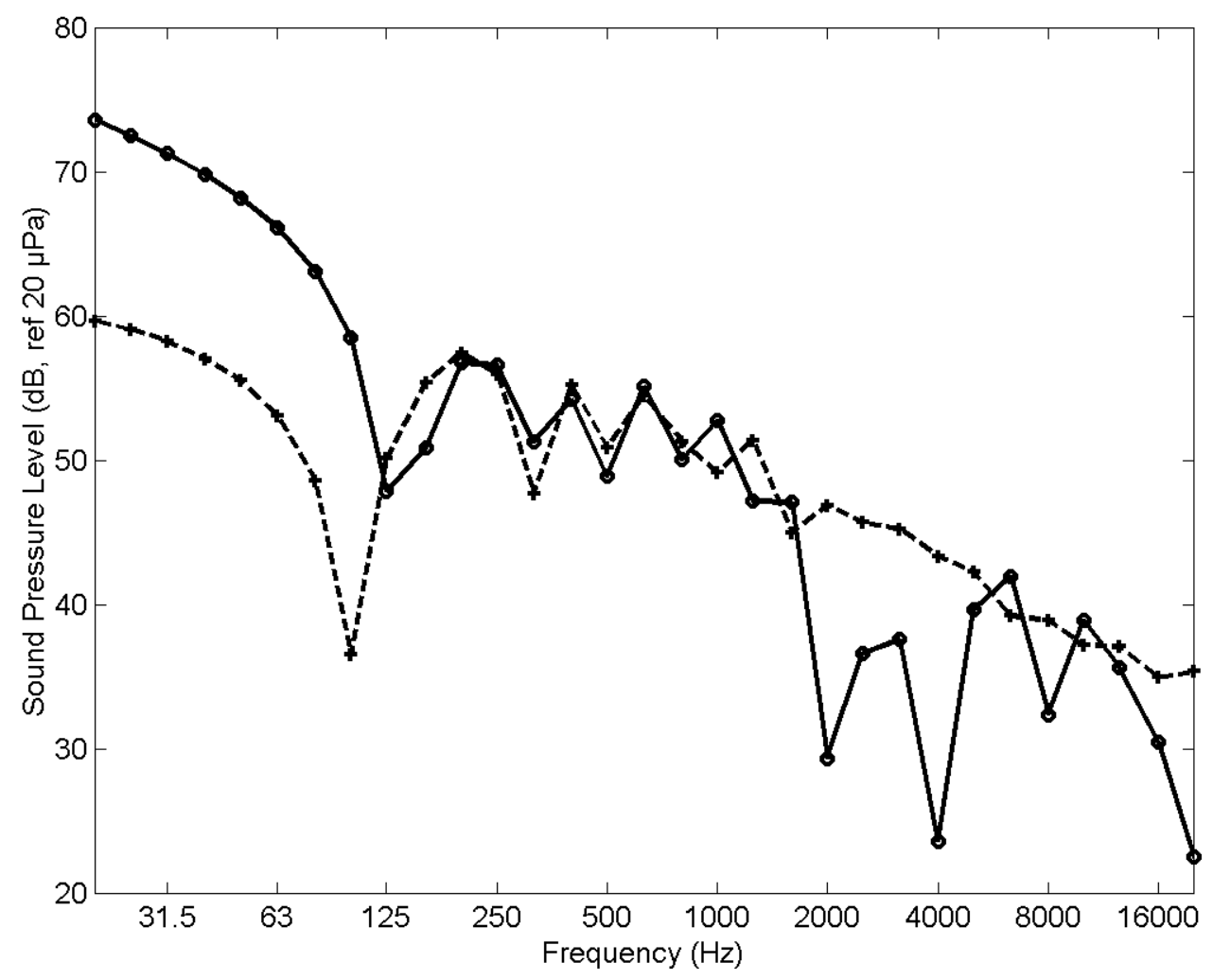

Figure 3.4: Comparison between results obtained using a beam tracing simulation and the K \& A equation from Muradali and Fyfe (1997). The solid line represents data obtained using Lam's method and the dashed line represents data obtained using RAYNOISE.

\section{$\underline{3.3 \text { Model Strategy and Assumptions }}$}

\subsubsection{Model Design}

Blueprints for the Portage Barrier Toll Collection facility were studied before construction of the software model. The key elements of the plaza are the ground, the tollbooths, the overhead canopy, and the supports for the canopy. The canopy supports were omitted from the model for simplicity. Diffraction about the diameter would cause the 
presence of the supports to be acoustically negligible. Figure 3.5 shows the baseline toll plaza model constructed using AutoCAD.

To prevent interior interactions from obscuring the analysis, the booths walls were assumed to be solid, without communication window openings. The ground, the tollbooths, and the overhead canopy were created as single element meshed surfaces within AutoCAD.

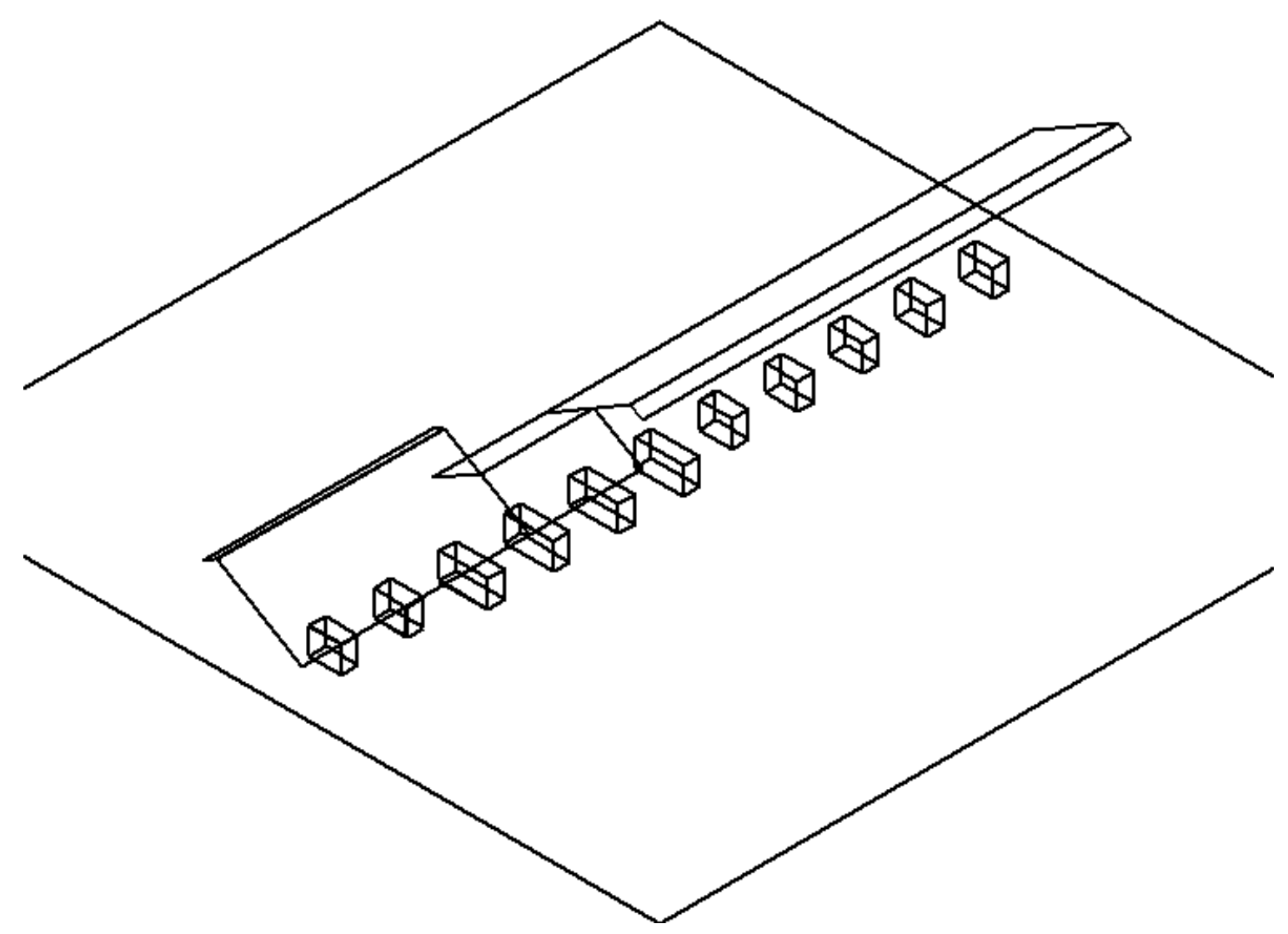

Figure 3.5: Baseline toll plaza model constructed in AutoCAD.

\subsubsection{Material Properties}

The acoustical properties of the construction materials for the key elements of the toll plaza were considered in the analysis. The exterior of the booths is comprised of 
aluminum and stainless steel. Corrugated metal sheets form the overhead canopy. The toll plaza vehicular roadway is surfaced in concrete.

The Sabine absorption coefficient, the diffusion coefficient, and the sound transmission loss specified over octave band center frequencies ranging from $63 \mathrm{~Hz}$ to 8000 $\mathrm{Hz}$ characterize different material properties. Material properties can be defined using a material database built into RAYNOISE, or by entering user-defined values. By stipulating both the absorption and the transmission coefficients of a surface, the reflection coefficient is defined. Diffusion characterizes the randomness of reflections in a non-specular way resulting from surface corrugations and roughness. The effects of surface diffusion were neglected, thus the diffusion coefficients for all materials were zero.

The acoustical properties of the two materials used are shown in Table 3.1. The values for the ground was assigned based on typical Sabine absorption of concrete and a transmission loss of concrete unfinished, $150 \mathrm{~mm}$ thick. To model the remaining metallic objects of the plaza, a Sabine absorption of zero and a transmission loss of sheet metal steel, $0.7 \mathrm{~mm}$ thick were used. The material properties defined were deemed appropriate. 


\begin{tabular}{|c|c|c|c|c|c|c|c|c|}
\hline \multicolumn{9}{|l|}{ Concrete } \\
\hline $\begin{array}{l}\text { Octave Band } \\
\text { Center } \\
\text { Frequency } \\
(\mathrm{Hz}) \\
\end{array}$ & 63 & 125 & 250 & 500 & 1000 & 2000 & 4000 & 8000 \\
\hline $\begin{array}{l}\text { Sabine } \\
\text { Absorption }\end{array}$ & 0.03 & 0.04 & 0.05 & 0.06 & 0.06 & 0.07 & 0.09 & 0.00 \\
\hline $\begin{array}{l}\text { Transmission } \\
\text { Loss }\end{array}$ & 0 & 35 & 40 & 49 & 55 & 60 & 65 & 0 \\
\hline \multicolumn{9}{|l|}{ Metal } \\
\hline $\begin{array}{l}\text { Octave Band } \\
\text { Center } \\
\text { Frequency } \\
(\mathbf{H z}) \\
\end{array}$ & 63 & 125 & 250 & 500 & 1000 & 2000 & 4000 & 8000 \\
\hline $\begin{array}{l}\text { Sabine } \\
\text { Absorption } \\
\end{array}$ & 0.00 & 0.00 & 0.00 & 0.00 & 0.00 & 0.00 & 0.00 & 0.00 \\
\hline $\begin{array}{l}\text { Transmission } \\
\text { Loss }\end{array}$ & 0 & 9 & 14 & 20 & 26 & 30 & 37 & 0 \\
\hline
\end{tabular}

Table 3.1: Material properties used to model elements of the Portage Barrier Toll Plaza.

\subsubsection{Diffraction Edges}

All of the vertical edges and all of the ceiling edges of the tollbooths were selected as diffraction edges. Diffraction around the toll booths causes sound waves to penetrate shadow zones that could not be reached by specular reflection. Since the canopy is not along the path between the sources and the receivers, diffraction of sound around the canopy edges does not contribute to the sound field. The remaining edges, the ground edges of the tollbooths, obviously cannot contribute to diffraction.

\subsubsection{Ambient Conditions}

The air medium was assumed to be homogeneous and at rest, at the mean temperature of 20 degree Celsius with a 50 percent relative humidity. Hence the speed of 
sound was 343.325 meters per second. The possible effects of temperature gradients and wind were not taken into account.

\subsubsection{Source Spectrum}

All sources were ideal spherical point sources, which are also called monopoles. The sound power level spectrum, position, and radiation pattern of the spherical point sources were specified. More complicated source patterns were built through the use of multiple point sources.

Semi-trucks may cause the highest noise levels. Semi-trucks have two distinct sound characteristics - idling and acceleration, each with a different level and frequency spectrum. To model the source characteristics of the semi-trucks, the procedures utilized in another prediction software package, FHWA Traffic Noise Model (TNM), were used (U. S. Department of Transportation, 1998). In TNM, heavy trucks are modeled using a pair of monopole sources. One source is located at the ground level and the other source is located 3.66 meters ( $12 \mathrm{ft}$ ) directly above the first. The A-weighted sound pressure level in one third octave bands for each monopole is specified at a distance of 15 meters from the source.

To model an idling semi-truck, TMN data for heavy trucks on average pavement traveling at $16.09 \mathrm{kph}(10 \mathrm{mph})$ under cruise throttle was used. To model an accelerating semi-truck, TMN data for heavy trucks at $32.19 \mathrm{kph}(20 \mathrm{mph})$ under full throttle were used. As input to the RAYNOISE model, TNM one third octave band sound pressure level data was converted to octave band sound power level data. The source values used are presented in Table 3.2.

The locations of the semi-truck sound sources are shown in Figure 3.6. In the center of each lane three semi-truck idle sources and one acceleration source were constructed. The first idling semi-truck was positioned central to the large toll booths and 
just off center of the small tollbooths. The remaining two idling semi-trucks were placed 3.05 meters $(10 \mathrm{ft})$ fore and aft of the center idling semi-truck. The accelerating semi-truck was placed 6.10 meters $(20 \mathrm{ft})$ aft the last idling semi-truck, consistent with normal traffic flow.

Each semi-truck source was modeled with an incoherent source pair similar to that used in TNM. The bottom source was assigned a uniform monopole radiation characteristic, an octave band power level spectrum, and source height of 0.15 meters $(0.5 \mathrm{ft})$ above the ground. A height of 0.15 meters was used instead of the TMN prescribed height of 0 meters, to prevent anomalous transmissions through the ground of the model. The top source was assigned a uniform monopole radiation characteristic, an octave band power level spectrum, and source height of 4.57 meters (15 ft) above the ground as prescribed in TNM.

\begin{tabular}{|l|l|l|l|l|l|l|l|l|}
\hline $\begin{array}{l}\text { Octave Band } \\
\text { Center } \\
\text { Frequency } \\
\text { (Hz) }\end{array}$ & $\mathbf{6 3}$ & $\mathbf{1 2 5}$ & $\mathbf{2 5 0}$ & $\mathbf{5 0 0}$ & $\mathbf{1 0 0 0}$ & $\mathbf{2 0 0 0}$ & $\mathbf{4 0 0 0}$ & $\mathbf{8 0 0 0}$ \\
\hline $\begin{array}{l}\text { Idle Low } \\
\text { Source (dB) }\end{array}$ & 74.5714 & 81.9378 & 85.1124 & 86.7804 & 88.7490 & 88.0464 & 86.4313 & 81.0199 \\
\hline $\begin{array}{l}\text { Idle High } \\
\text { Source (dB) }\end{array}$ & 76.1521 & 83.5185 & 86.6931 & 88.3611 & 87.7603 & 85.5476 & 83.9325 & 78.5211 \\
\hline $\begin{array}{l}\text { Acceleration } \\
\text { Low Source } \\
\text { (dB) }\end{array}$ & 77.8139 & 86.3631 & 89.6019 & 92.6019 & 95.6703 & 94.2228 & 90.1610 & 83.2050 \\
\hline $\begin{array}{l}\text { Acceleration } \\
\text { High Source } \\
\text { (dB) }\end{array}$ & 79.2157 & 87.7649 & 91.0037 & 94.0037 & 94.8572 & 92.2801 & 88.2184 & 81.2623 \\
\hline
\end{tabular}

Table 3.2: The semi-truck source spectrum, sound power level. 


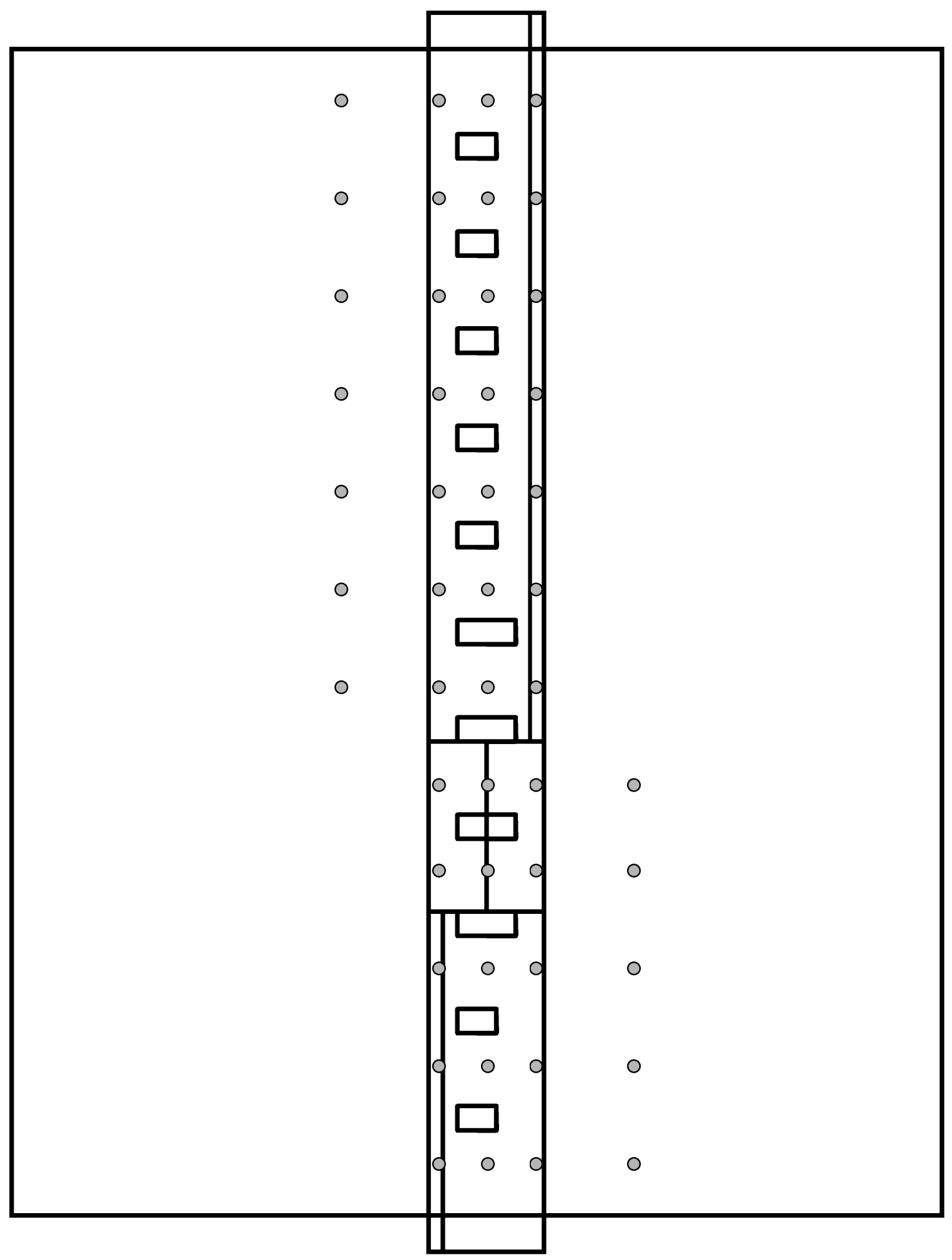

Figure 3.6: $\quad$ Location of semi-truck sound sources within the basic model of the toll plaza, top view. 


\subsubsection{Receiver Locations}

Receiver locations (microphone positions) must be specified. More complicated receiver geometries are constructed by meshing a surface where the nodal locations created by the meshing process define the location of point receivers.

At the toll plaza, the receivers of interest are tollbooth attendants' ears. To model the noise experienced by the tollbooth attendants, receiver planes were defined at the locations of the tollbooth open communication windows. To prevent anomalous transmissions through tollbooth walls, the receiver point mesh was placed 0.15 meters $(0.5 \mathrm{ft})$ in front of the tollbooth surface. Figure 3.7 shows a diagram of the receiver plane locations. The number of nodal points affects the level of resolution and the computational time. As a compromise between efficiency and accuracy, a four point mesh for each receiver field was used. Four receiver points, one in each corner, were positioned at the window location. 


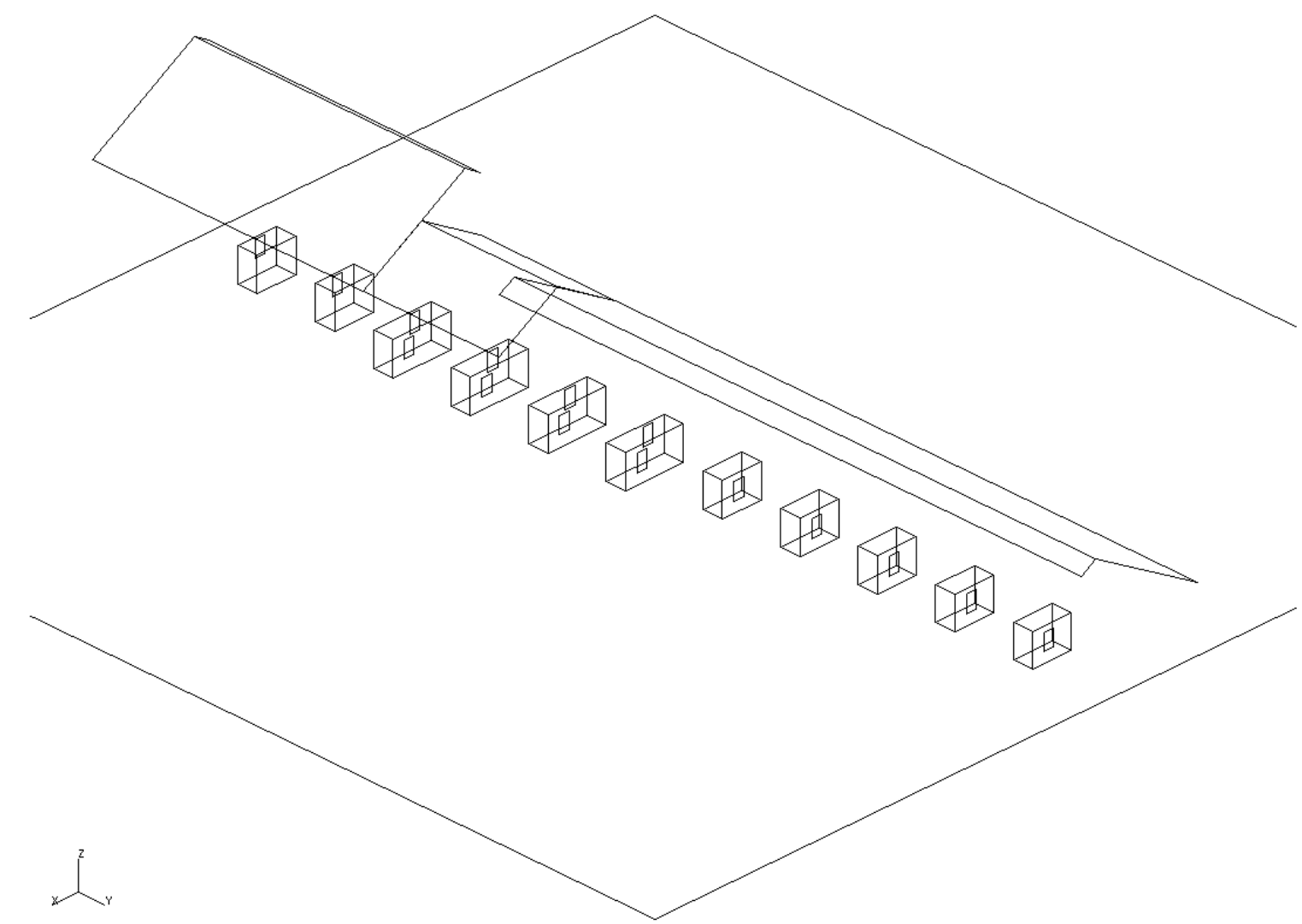

Figure 3.7: Location of receiver points within the baseline model of the toll plaza.

\subsubsection{Calculation Procedures}

There are two phases of RAYNOISE calculation. The first phase is more computationally intensive and involves mapping the beam paths. The second phase involves postprocessing the results into meaningful acoustic parameter. The first step in the analysis is to select the sources and the frequencies of interest. Then calculation parameters need to be defined. The calculation parameters are divided into the following four classes: propagation, echogram, histogram, and general. Information about the calculation parameters can be found in the RAYNOISE Users Manual (LMS Numerical Technologies, 1998, chap. 9.5). Only the propagation and general subsets are important to the type of analysis used. The default propagation settings were used: 2000 rays, $10^{\text {th }}$ order reflection, 
2000 ms time window, and $90 \mathrm{~dB}$ dynamic range. The number of rays defines the number of beams used to model each source. The reflection order defines the number of beam reflections tracked in the analysis. The duration of the time window is the period in which echoes are calculated for an echogram study. The dynamic range specifies the level at which a beam is abandoned. In a convergence study, the number of rays was increased to 200,000 from the above value to investigate the accuracy of the predictions. For the test case, there was no discernable difference. The selected general settings consisted of the settings: no diffusion calculation, diffraction calculation, no tail compensation, and the triangular beam method. The diffusion and diffraction settings are consistent with previously defined material properties. Tail correction has no physical meaning in a exterior model such as this, and should not be used as explained in the RAYNOISE User's Manual (LMS Numerical Technologies, 1998, chap. 3.1.6). The triangular beam method was selected over the conical beam method, due to the higher accuracy of the triangular beam method as explained in the RAYNOISE User's Manual Section (LMS Numerical Technologies, 1998, chap. 3.1.4).

As a final step, RAYNOISE environmental variables were investigated, to uncover pertinent parameters that are not available in the standard calculation menus (LMS Numerical Technologies, 1998, chap. 11.8). During this investigation, the HIGHACCURCY parameter was chosen to increase the accuracy of the model results. This additional test is used to avoid contributions of false image sources (LMS Numerical Technologies, 1998, chap. 3.1.5 and 11.8.3.34).

\subsubsection{Data Analysis}

To better utilize the RAYNOISE generated data, the results were exported into a free format text file. A MATLAB function was constructed to read the free format text file and 
convert the data into a matrix. Once the data is in a matrix form it can be manipulated to thoroughly investigate the noise conditions at the plaza.

\subsection{Summary}

Two validation cases demonstrated that the RAYNOISE has an adequate level of accuracy to simulate the noise conditions at the toll plaza. Based upon analysis of the blueprints, physical elements such as the ground, tollbooths and overhead canopy were modeled. Semi-truck sources and receivers at the communication window location were defined. Detailed parameters affecting the type of calculation were reviewed.

The ground work for RAYNOISE modeling has been well established in this chapter. The next chapter reports on the results of various RAYNOISE case studies. In these case studies, the effects of architectural changes such as: the treatment of existing overhead canopy with sound absorbing material, the implementation of partial barriers with absorbing surface treatments, and the placement of sound absorbing material on the walls of the tollbooths will be explored. 


\section{DESIGN STUDY}

\section{$\underline{4.1 \text { Introduction }}$}

RAYNOISE is well suited for evaluation of noise control strategies that involve architectural changes. Reasonable implementations of passive noise control solutions for the toll booth application discussed in Chapter 2 include sound absorbing treatment of the existing overhead canopy, treatment of the tollbooth walls with sound absorbing material, and implementation of partial barriers with absorbing surface treatments. Modeling of these passive noise control solutions are discussed in this chapter.

\section{$\underline{4.2 \text { Model Configurations }}$}

Five toll plaza models were created to test the performance of reasonable passive noise control approaches. The location and properties of the ground, sources, and receivers remained constant for all of the models as described in Chapter 3. The noise control strategy was the only change from model to model

\subsubsection{Reference Model}

A model of the existing toll plaza was created as described in Chapter 3. The model is shown in Figure 4.1. In this model the ground, the tollbooths, and the overhead canopy 
were included. This model was constructed as the reference against which the performance of all alterations would be assessed.

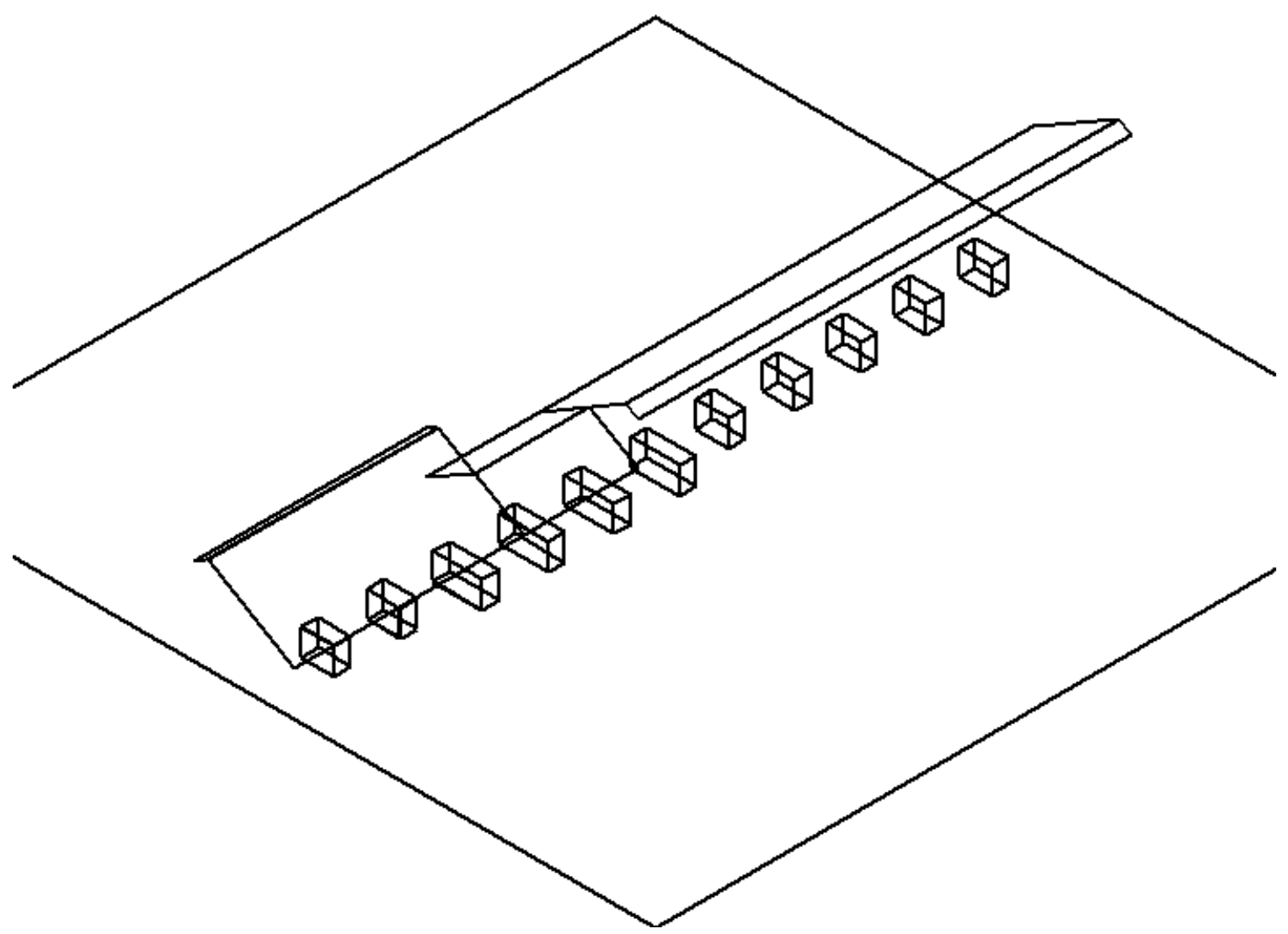

Figure 4.1: Model of the Portage Barrier Toll Plaza used as the reference condition.

\subsubsection{Absorbing Canopy}

The canopy lies above both the sources and the receivers at the toll plaza. The canopy may increase the sound level at the receiver location by creating additional, overhead reflections. To model a canopy with perfect sound absorbing treatment, the canopy was removed from the model. A diagram of the model is shown in Figure 4.2. This model represents the best achievable performance obtainable with surface treatments. 


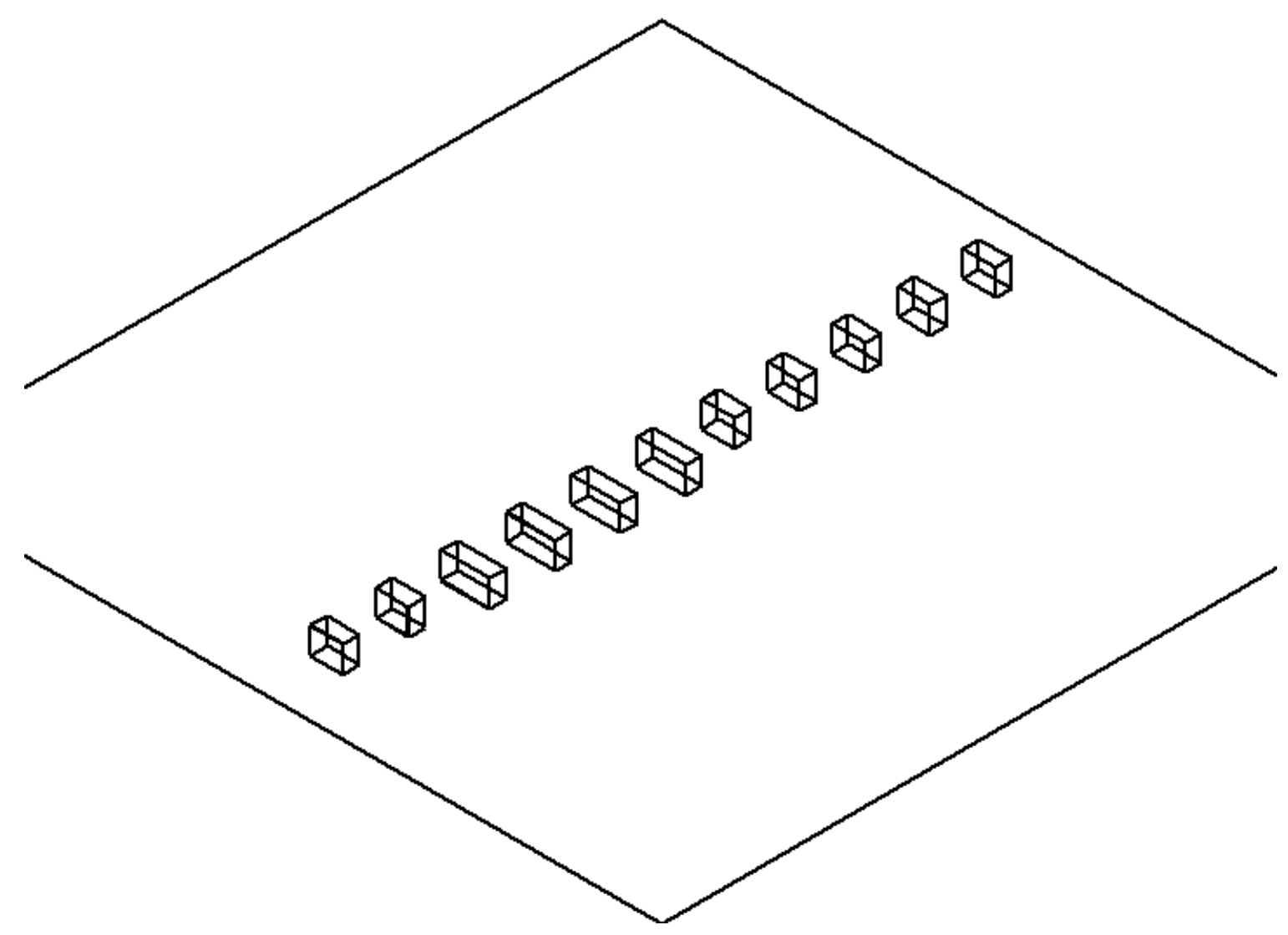

Figure 4.2: Toll plaza model with the canopy removed to simulate a perfectly absorbing canopy.

\subsubsection{Absorbing Partial Barriers}

Reflecting barriers could be used to isolate sound sources in one lane of traffic from the other lanes. Application of absorbing treatment on the barriers is used to reduce the energy of reflections off of the barriers.

The test configuration included two dimensional barriers measuring 1.37 meters (4.5') long by 1.21 meters (4') tall set 0.15 meters $\left(0.5^{\prime}\right)$ away from the east side of the tollbooths. Barriers measuring 1.37 meters (4.5') long by 1.21 meters (4') tall were placed 0.15 meters $\left(0.5^{\prime}\right)$ away from the west side of the larger booths. Barriers measuring 2.90 meter $\left(9.5^{\prime}\right)$ by 1.21 meters ( $\left.4^{\prime}\right)$ tall were placed 0.15 meters $\left(0.5^{\prime}\right)$ away from the west side 
of the small booths. The barriers were centered about the booths and parallel to the lanes of traffic. A diagram of the model is shown in Figure 4.3. Often toll employees must cross lanes of traffic. In such conditions, a clear view of the traffic lanes is necessary for safety. The height of the barrier was selected to avoid obstructing the view of traffic. The length of the barriers was selected to conform to the existing canopy supports.

The material properties of the barriers were assigned using the RAYNOISE material database. A listing of the material properties is shown in Table 4.1. To model a concrete partial barrier with sound absorbing surface treatment, the Sabine absorption of mineral wool, 0.05 meters thick and the transmission loss of concrete unfinished, 0.15 meters thick were used. These material properties are typical of the actual conditions of a high quality implementation. The top and side edges of the barriers were designated diffraction edges to model sound diffraction due to the barriers. 


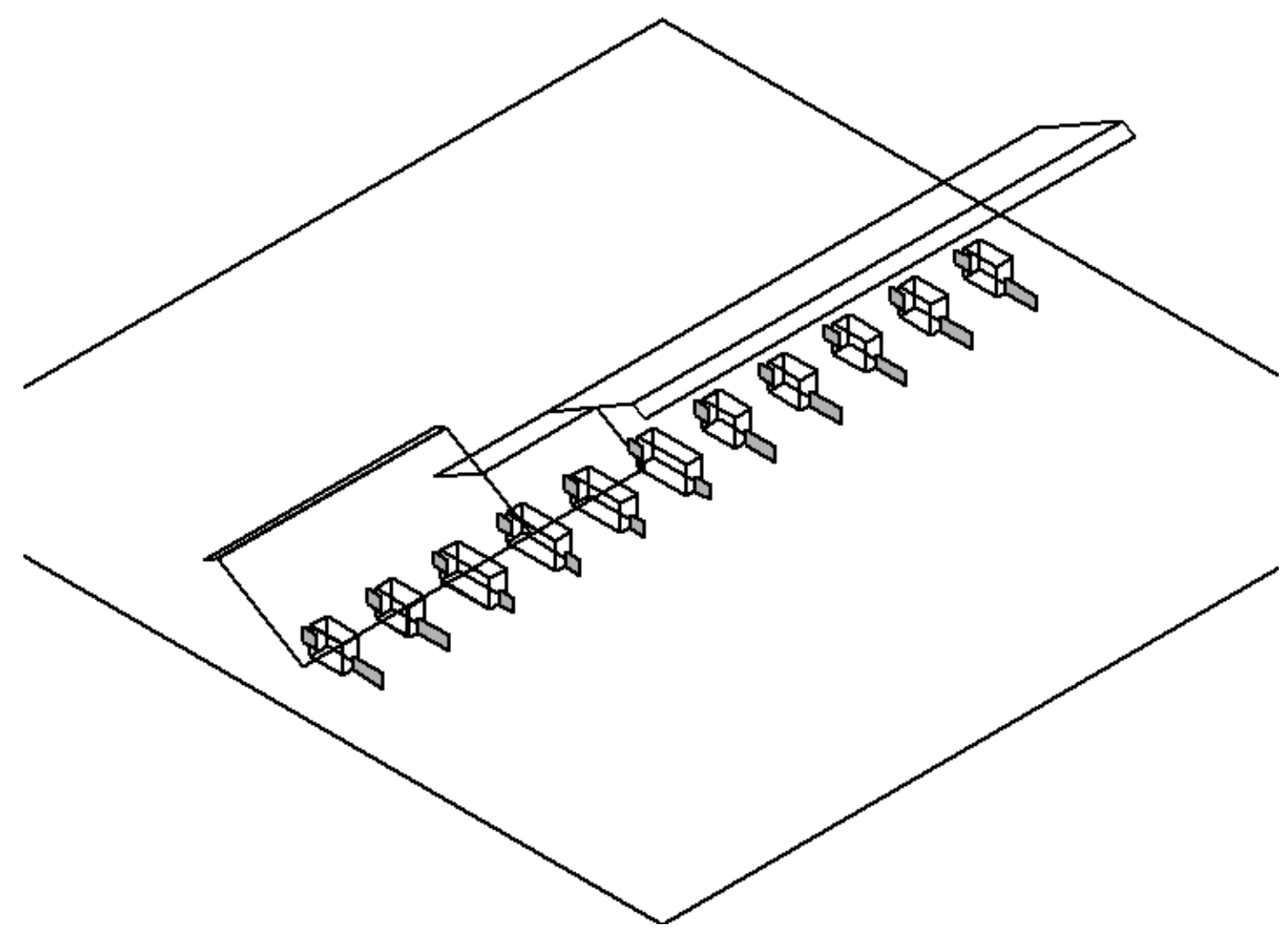

Figure 4.3: Toll plaza model with sound absorbing partial barriers.

\begin{tabular}{|l|l|l|l|l|l|l|l|l|}
\hline $\begin{array}{l}\text { Octave Band } \\
\text { Center } \\
\text { Frequency } \\
\text { (Hz) }\end{array}$ & $\mathbf{6 3}$ & $\mathbf{1 2 5}$ & $\mathbf{2 5 0}$ & $\mathbf{5 0 0}$ & $\mathbf{1 0 0 0}$ & $\mathbf{2 0 0 0}$ & $\mathbf{4 0 0 0}$ & $\mathbf{8 0 0 0}$ \\
\hline $\begin{array}{l}\text { Sabine } \\
\text { Absorption }\end{array}$ & 0.40 & 0.39 & 0.45 & 0.56 & 0.59 & 0.61 & 0.55 & 0.50 \\
\hline $\begin{array}{l}\text { Transmission } \\
\text { Loss }\end{array}$ & 0 & 35 & 40 & 49 & 55 & 60 & 65 & 0 \\
\hline
\end{tabular}

Table 4.1: $\quad$ Table of the material characteristics assigned for the partial barriers with sound absorbing treatment. 


\subsubsection{Absorbing Treatment for the Tollbooths}

To further explore passive noise control solutions, treatment of the tollbooth surfaces with sound absorbing material was evaluated. Since tollbooths are parallel to one another sound may reflect between adjacent booth walls. Similarly, semi-truck trailers have reflective surfaces that are parallel to the tollbooth faces. By treating the tollbooth surface with sound absorbing material, some of this reflected energy is absorbed.

The material property for the absorbing tollbooth surfaces was assigned using the RAYNSOISE material database. The material property is shown in Table 4.2. To model a metal tollbooth with sound absorbing surface treatment, a Sabine absorption of mineral wool, 0.05 meters thick and a transmission loss of sheet metal steel, $0.7 \mathrm{~mm}$ thick were used. This material property is typical possible for a realistic application condition. The material property was assigned to all faces of each tollbooth.

\begin{tabular}{|l|l|l|l|l|l|l|l|l|}
\hline $\begin{array}{l}\text { Octave Band } \\
\text { Center } \\
\text { Frequency }\end{array}$ & $\mathbf{6 3}$ & $\mathbf{1 2 5}$ & $\mathbf{2 5 0}$ & $\mathbf{5 0 0}$ & $\mathbf{1 0 0 0}$ & $\mathbf{2 0 0 0}$ & $\mathbf{4 0 0 0}$ & $\mathbf{8 0 0 0}$ \\
\hline $\begin{array}{l}\text { Sabine } \\
\text { Absorption }\end{array}$ & 0.40 & 0.39 & 0.45 & 0.56 & 0.59 & 0.61 & 0.55 & 0.50 \\
\hline $\begin{array}{l}\text { Transmission } \\
\text { Loss }\end{array}$ & 0 & 9 & 14 & 20 & 26 & 30 & 37 & 0 \\
\hline
\end{tabular}

Table 4.2: Material characteristics assigned for sound absorbing tollbooth walls.

\subsubsection{Combined Modifications}

The performance of the individual passive control approaches is not necessarily additive. Since the application of sound absorbing material in one location may prevent energy from reaching another location, the combined noise control approaches was tested as a separate case. To test the combined effects of a perfectly absorbing canopy, absorbing 
partial barriers, and absorbing tollbooth surfaces, a final design model was created. The model is shown in Figure 4.4.

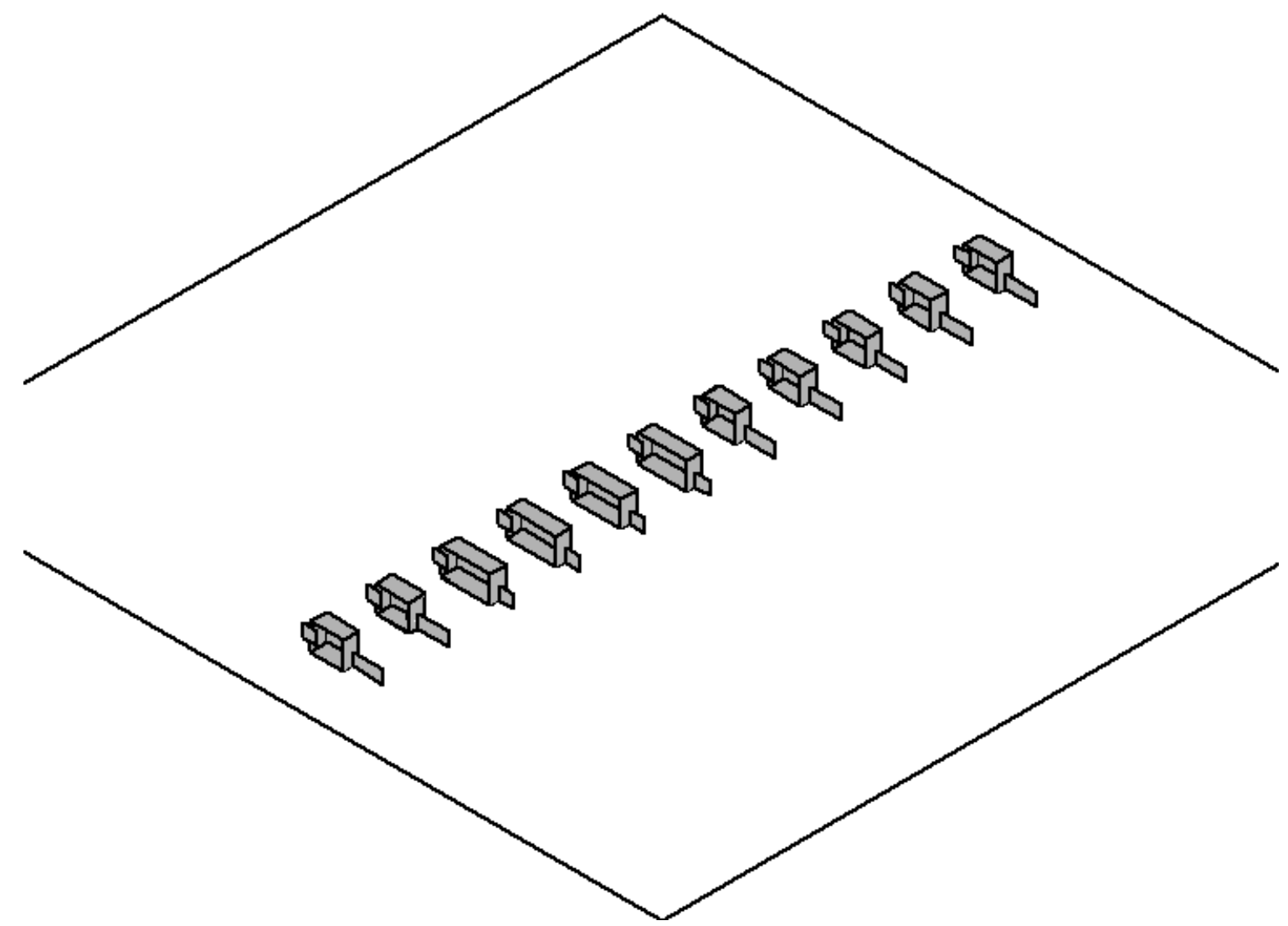

Figure 4.4: Toll plaza model with perfectly absorbing canopy, sound absorbing partial barriers, and absorbing treatment on the tollbooth walls.

\subsection{Results and Analysis}

As described in Chapter 3, the RAYNOISE models are processed for one active source pair at a time and the results are exported to a file. Processing each model in RAYNOISE involved computing the response of 48 source pairs for 9 octave band center frequencies at 15 receiver planes each with 4 data points. Therefore the amount of data 
generated per case is quite extensive. The files are imported into MATLAB where the data can be more readily reduced, analyzed, and compared.

In preliminary tests, it was demonstrated that the variation of sound pressure level across each receiver plane (tollbooth operator window location) was small. To arrive at a single representative sound pressure level for each window location, the energy of the four data points for each window location was averaged using MATLAB.

The insertion loss for a noise control technique was calculated by subtracting the level of the modified model from the level of the reference model for each corresponding case. By this method, the reduction of noise at the plaza due to each passive technique can be assessed.

\subsubsection{Frequency Dependence}

The first stage of analysis involved evaluation of the insertion loss performance as a function of frequency. Since the RAYNOISE analysis was performed using a single source pair at a time, multiple vehicles can be simulated by summing the corresponding power associated with each vehicle source case. As a starting point for this analysis, all the vehicle sources were activated. This condition is used to model full traffic which is the worst noise condition at the toll plaza. The sound pressure level and the insertion loss at each of the window locations was analyzed as a function of frequency. Representative cases are shown in Figures 4.5 and 4.6. Other results are collected in the Appendix.

Throughout all 15 window locations, there was a high degree of uniformity with respect to the insertion loss results. The insertion loss did not vary more than $1 \mathrm{~dB}$ across all nine octave bands for each window location. Furthermore the slight variation for all window locations followed a consistent pattern. 
One decibel is considered the minimum difference in sound pressure level that can be detected by human subjects. Since the frequency dependence was at most $1 \mathrm{~dB}$ at each window location and often below that, the slight insertion loss variation with respect to frequency is insignificant. Thus, the overall sound pressure level was used in further model analysis. The overall sound pressure level is calculated by summing the power of the octave frequency band predictions. Use of the overall sound pressure level results in a single, frequency independent value which reduces the number of model parameters and makes comparison of the results more straightforward.

While this test was performed to explore frequency dependence of insertion loss, information about the performance of the noise control techniques can be gained. The partial barriers appeared to have no perceivable effect. All of the predicted insertion loss data were a fraction a dB. The perfectly absorbing sound treatment on the canopy often yielded less than $1 \mathrm{~dB}$ of insertion loss with a maximum of $1.5 \mathrm{~dB}$ for select frequency bands. Treatment of the booths produced an insertion loss which ranged from a fraction of a $\mathrm{dB}$ to a maximum of $2 \mathrm{~dB}$. The combined noise control solutions resulted in an insertion loss that ranged between 1 to $3.5 \mathrm{~dB}$. A more in depth analysis will follow with subsequent studies. 


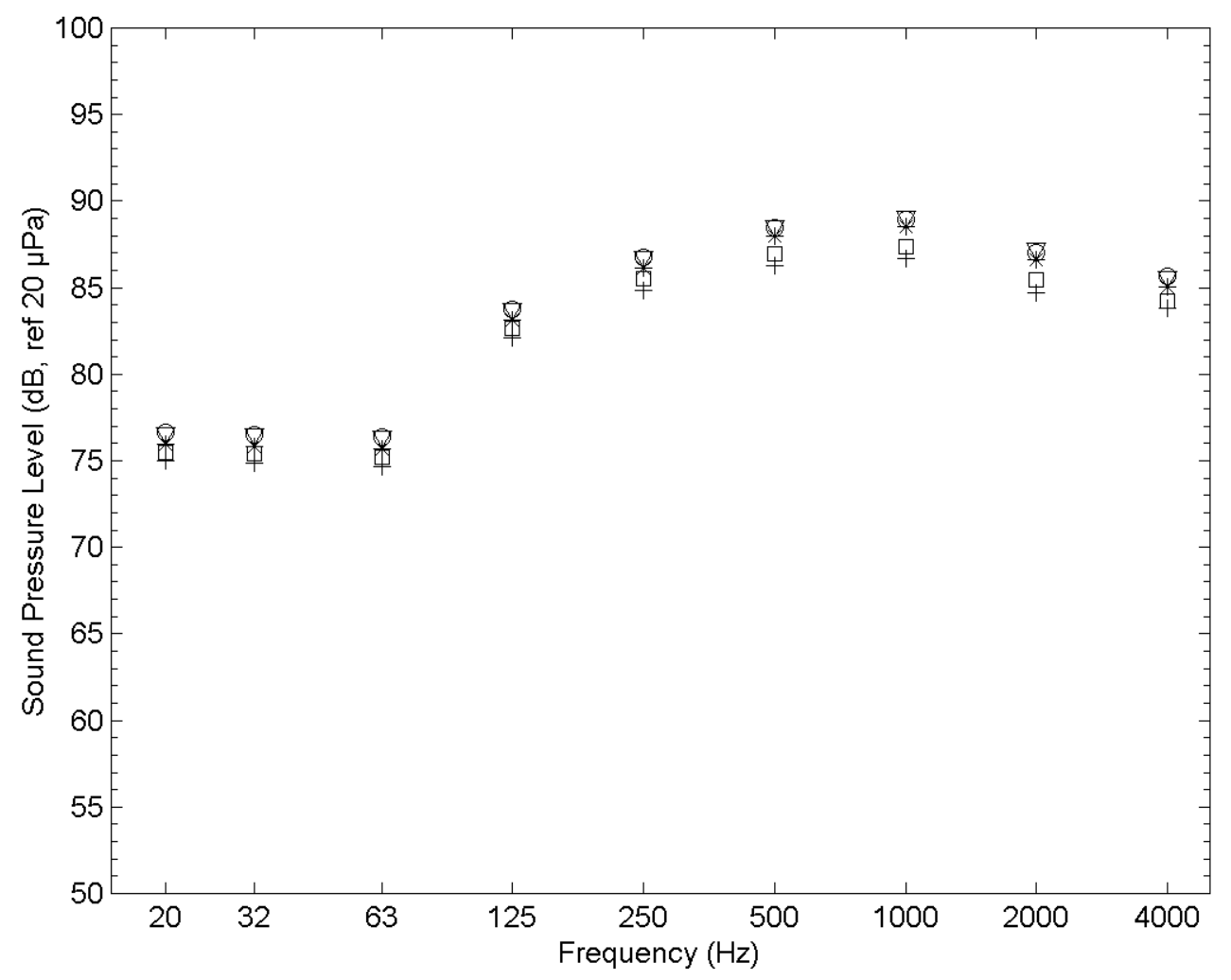

Figure 4.5: Sound pressure level of all sources active as a function of frequency at the fourth window location to the north of the plaza. $\nabla$ level of existing toll plaza; * level with absorbing canopy; O level with absorbing barriers; level with absorbing barriers; + level with all modifications. 


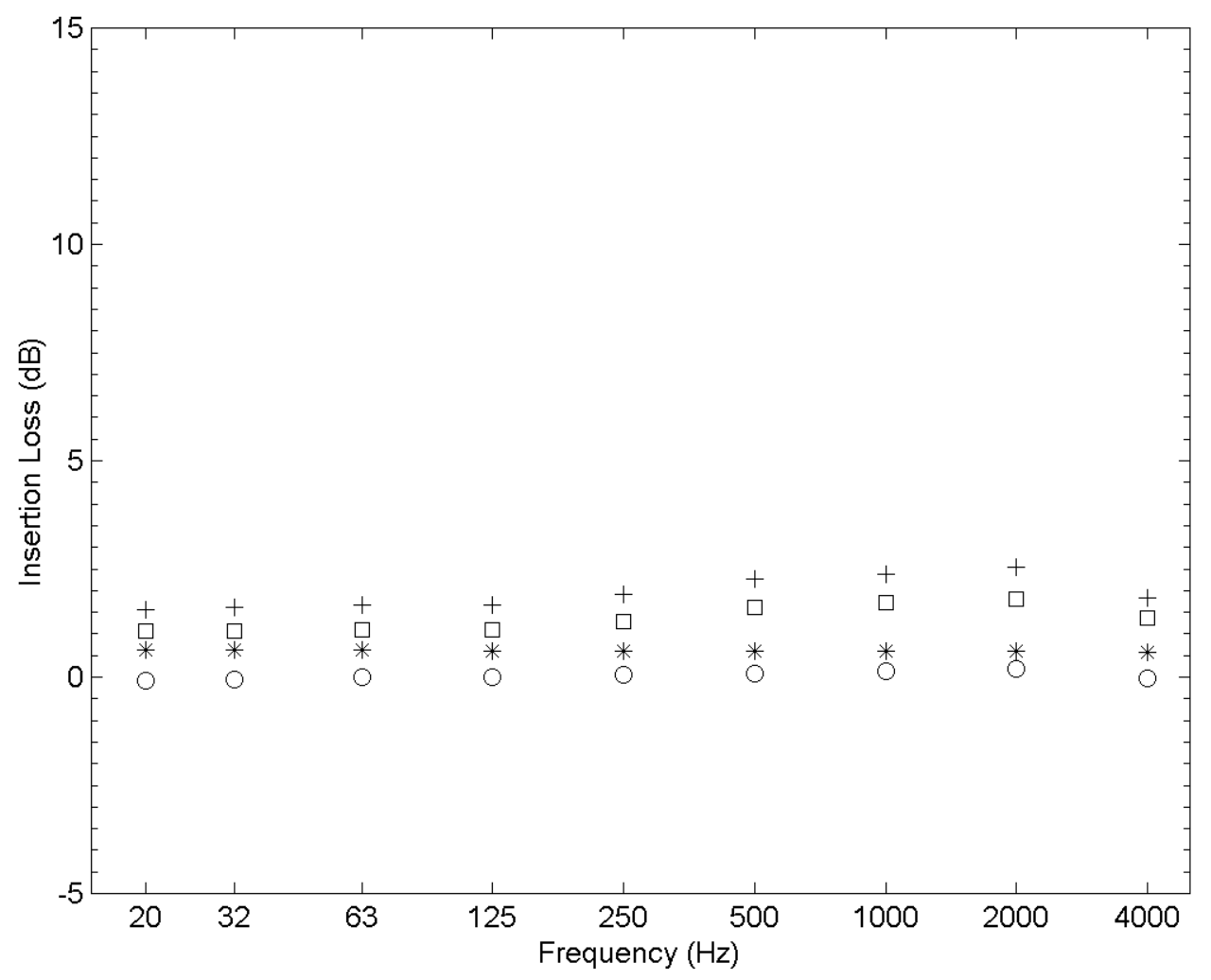

Figure 4.6: Insertion loss of all sources active as a function of frequency at the fourth window location to the North of the plaza. * insertion loss of absorbing canopy; O insertion loss of absorbing barriers; insertion loss of absorbing barriers; + insertion loss of all modifications.

\subsubsection{Investigating Individual Sources}

In the prior section, all sources were activated as a starting point in the investigation of noise control solutions. Beginning to delving into the details of noise control performance, individual sources were activated and the overall level and insertion loss was analyzed as a function of window position for each of the 48 source pairs. Representative cases are shown in Figures 4.7 and 4.8. The results for the remaining source pair cases can be found in the Appendix. 
For the idle sources, which are close to the tollbooth location, the level was to least 5 $\mathrm{dB}$ and often $10 \mathrm{~dB}$ higher for windows in the lane where the source is located than for windows in other lanes. For the acceleration sources, which are farther from the tollbooth location, the level was around 3 to $5 \mathrm{~dB}$ higher for windows in the lane where the source is located than for windows in the adjacent lanes. The idle sources are relatively much closer to the windows in the immediate lane than to any other window location. Since the acceleration sources are not significantly closer to the windows in the active lane than to the windows in the adjacent lanes, the level difference is not as dramatic.

The results imply that distance of the source to the receiver is an important factor in the sound pressure level at the receiver location. Under normal plaza conditions, where each lane is serving at least one vehicle, the sound field at the window locations appears to be dominated by the sound source in the lane being served. This implies that the sources in one lane often have little effect upon the level in another lane. To adequately deal with noise due to normal traffic conditions, the solution has to address noise control in the active lane.

The analysis discussed in this chapter used semi-truck sources which are the most extreme noise source at the toll plaza and of greatest interest. In reality, patrons of the toll roads often use passenger vehicles. Since the level of passenger vehicles is in general much lower than that of semi-trucks, the direct field may be less important in certain circumstances where passenger vehicles coexist with semi-trucks. If the immediate lane serves a passenger vehicle and the adjacent lane serves a semi-truck, the sound field in the immediate lane may have a significant contribution from the energy of the semi-truck. This energy would propagate to the immediate lane through reflection. Therefore absorbing material may be more significant in this situation since the sound field has a greater importance on reflected energy.

The mixed source scenario can be assessed with the test data of this section. Examining the insertion loss at receiver locations adjacent to the lane with the active source 
pair would be an idealized simulation of a semi-truck and a passenger vehicle in side by side lanes. The partial barriers appeared to have an insertion loss than ranged from $-2.5 \mathrm{~dB}$ to 2 $\mathrm{dB}$ in the adjacent lanes. The perfectly absorbing sound treatment on the canopy yielded 0 $\mathrm{dB}$ to $4.5 \mathrm{~dB}$ of insertion loss in the adjacent lanes. Treatment of the booths produced an insertion loss which ranged between $0 \mathrm{~dB}$ to $2.5 \mathrm{~dB}$ for the adjacent lanes. The combined noise control solutions resulted in an insertion loss that ranged between $-2 \mathrm{~dB}$ to $7 \mathrm{~dB}$ in the adjacent lanes.

Furthermore, in such a situation were the immediate lane serves a passenger vehicle, the noise level is much lower than when the immediate lane serves a semi-truck. Due to the lower level, this situation does not exhibit the primary concerns of this research study such as fatigue, hearing damage, and low speech intelligibility. 


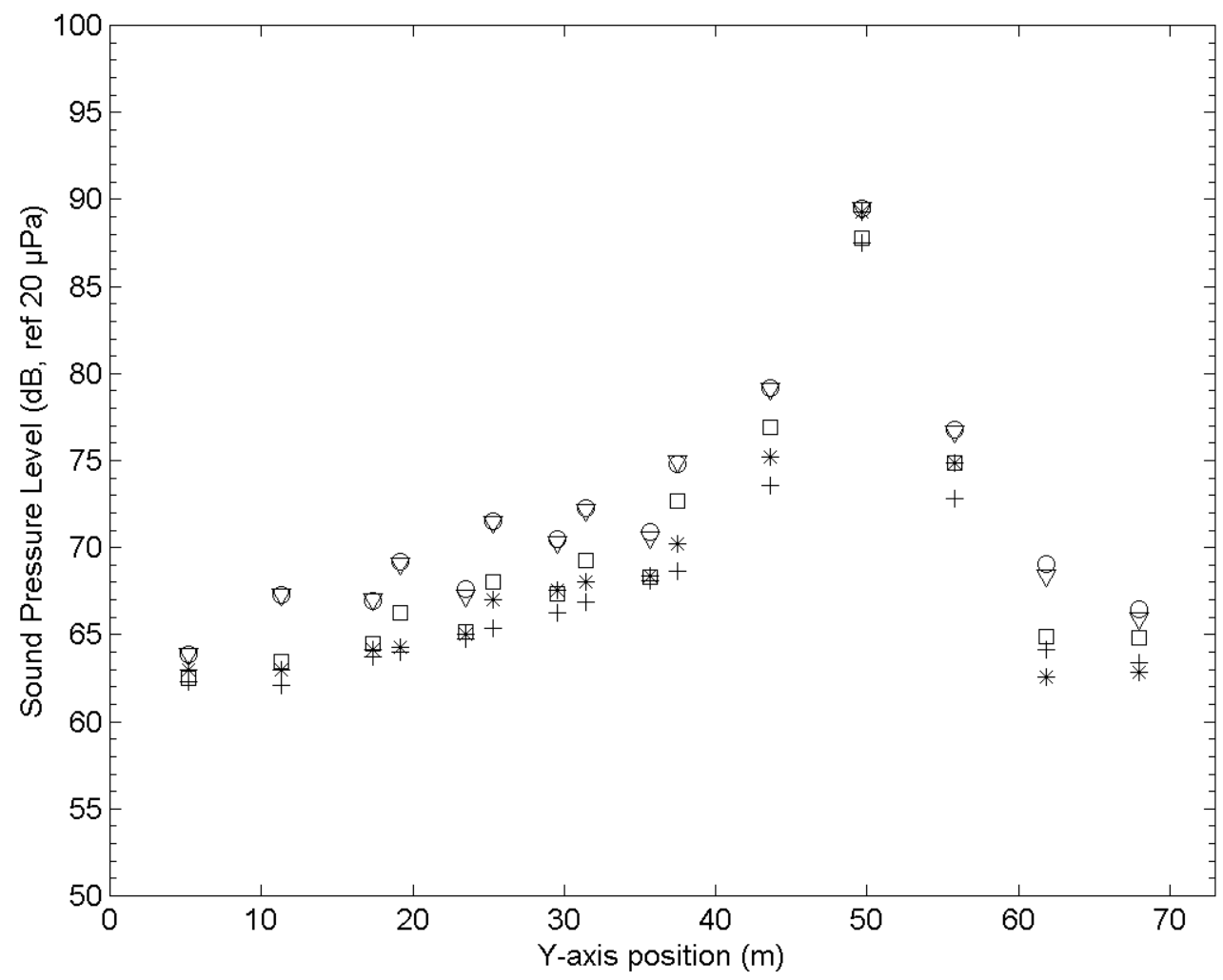

Figure 4.7: Overall sound pressure level for an individual source pair as a function of window location. Active source pair is the middle idle semi-truck located in fourth lane from the north. $\nabla$ level of existing toll plaza; * level with absorbing canopy; O level with absorbing barriers; level with absorbing barriers; + level is all modifications. 


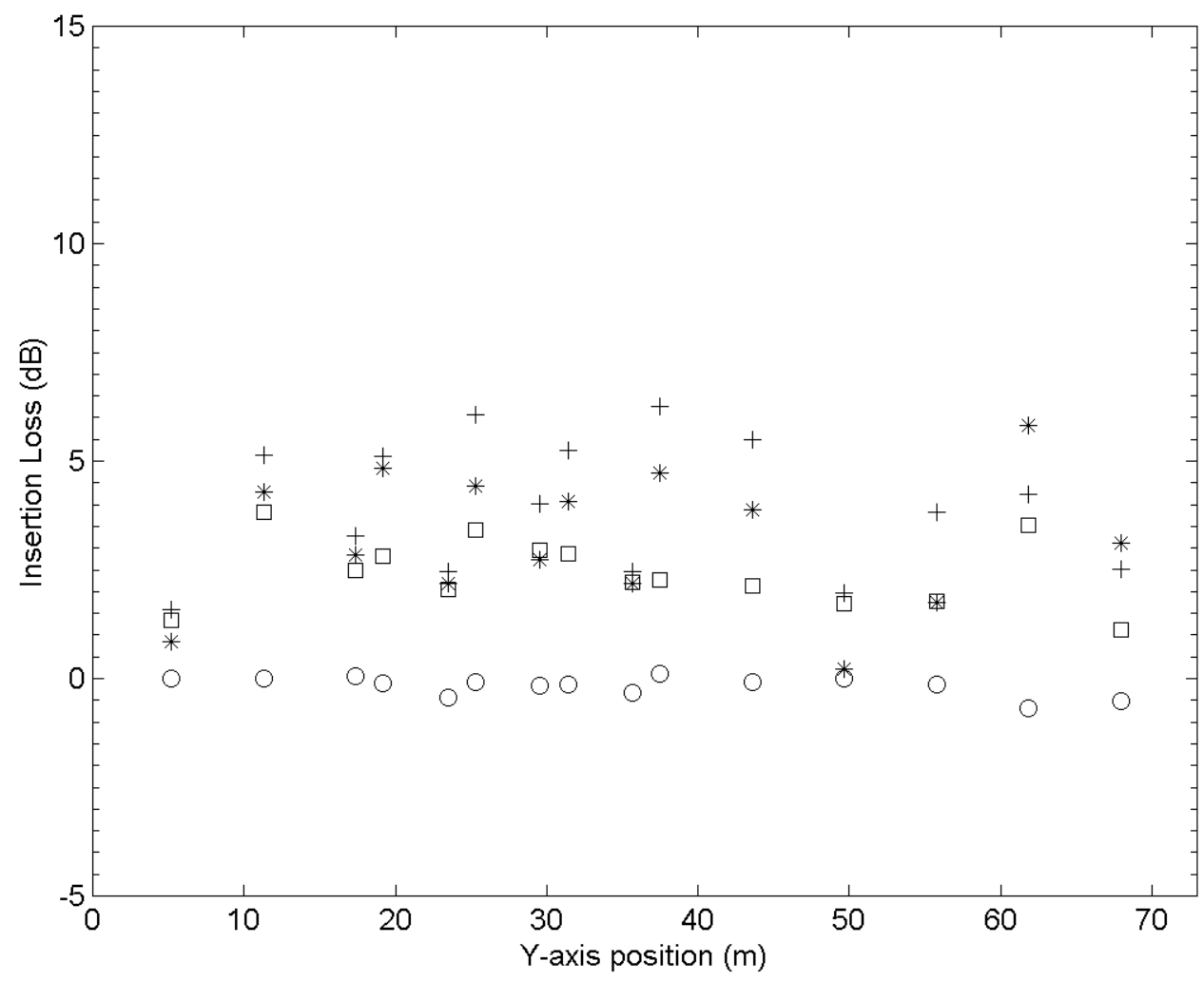

Figure 4.8: Insertion loss for an individual source pair as a function of window location. Active source pair is the middle idle semi-truck located in fourth lane from the north. * insertion loss of absorbing canopy; O insertion loss of absorbing barriers; insertion loss of absorbing barriers; + insertion loss of all modifications.

\subsubsection{Performance of Passive Techniques}

To further evaluate the performance of the passive techniques, all sources were activated in MATLAB. This represents the worst noise conditions, but a realistic condition when the toll plaza is crowded. The overall level and the insertion loss were evaluated as a function of window position. The results are shown in Figures 4.9 and 4.10.

The partial barriers appeared to have no perceivable effect. All of the predicted insertion loss data was a fraction of a $\mathrm{dB}$. The sound absorption of the barriers seemed to be offset by the additional sound reflections and diffraction. Similarly, the perfectly 
absorbing sound treatment on the canopy yielded less than $1 \mathrm{~dB}$ of insertion loss for the majority of the window locations. The negligible performance of the perfectly absorbing canopy can explained by considering the path length. The path length from the source to the receiver directly is relatively short. Sound waves that strike the canopy and reflect back to the receive have traveled a substantially greater distance. Due to spherical spreading, pressure is reduced by an inverse law and energy is reduced by an inverse square law. Therefore the level due to the reflected sound from the canopy becomes negligible when compared to the direct field. For treatment of the booths the insertion loss was almost $2 \mathrm{~dB}$ for the majority of the window locations.

Of all the noise control solutions tested, the combined approach yielded the highest performance. With all noise control techniques combined, the insertion loss was typically 2.5 to $3 \mathrm{~dB}$. Since this combined noise control configuration addressed treatment of every aspect of the toll plaza except the ground reflections, it further demonstrates the importance of the direct field. For the combined approach, the direct field accounted for all but $3 \mathrm{~dB}$ of the sound pressure level. 


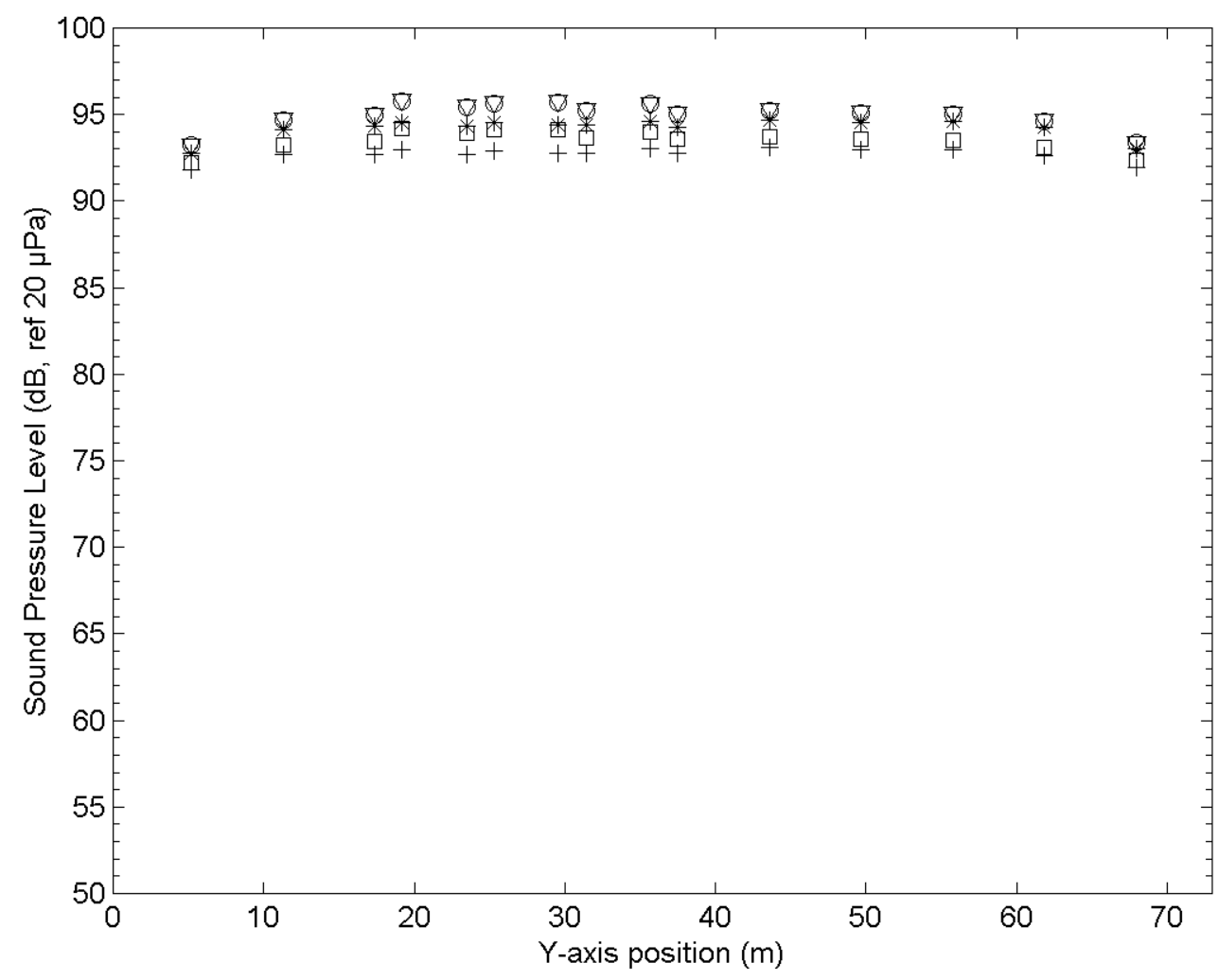

Figure 4.9: Overall level of all sources active as a function of window position. $\nabla$ level of existing toll plaza; * level with absorbing canopy; O level with absorbing barriers; level with absorbing barriers; + level is all modifications. 


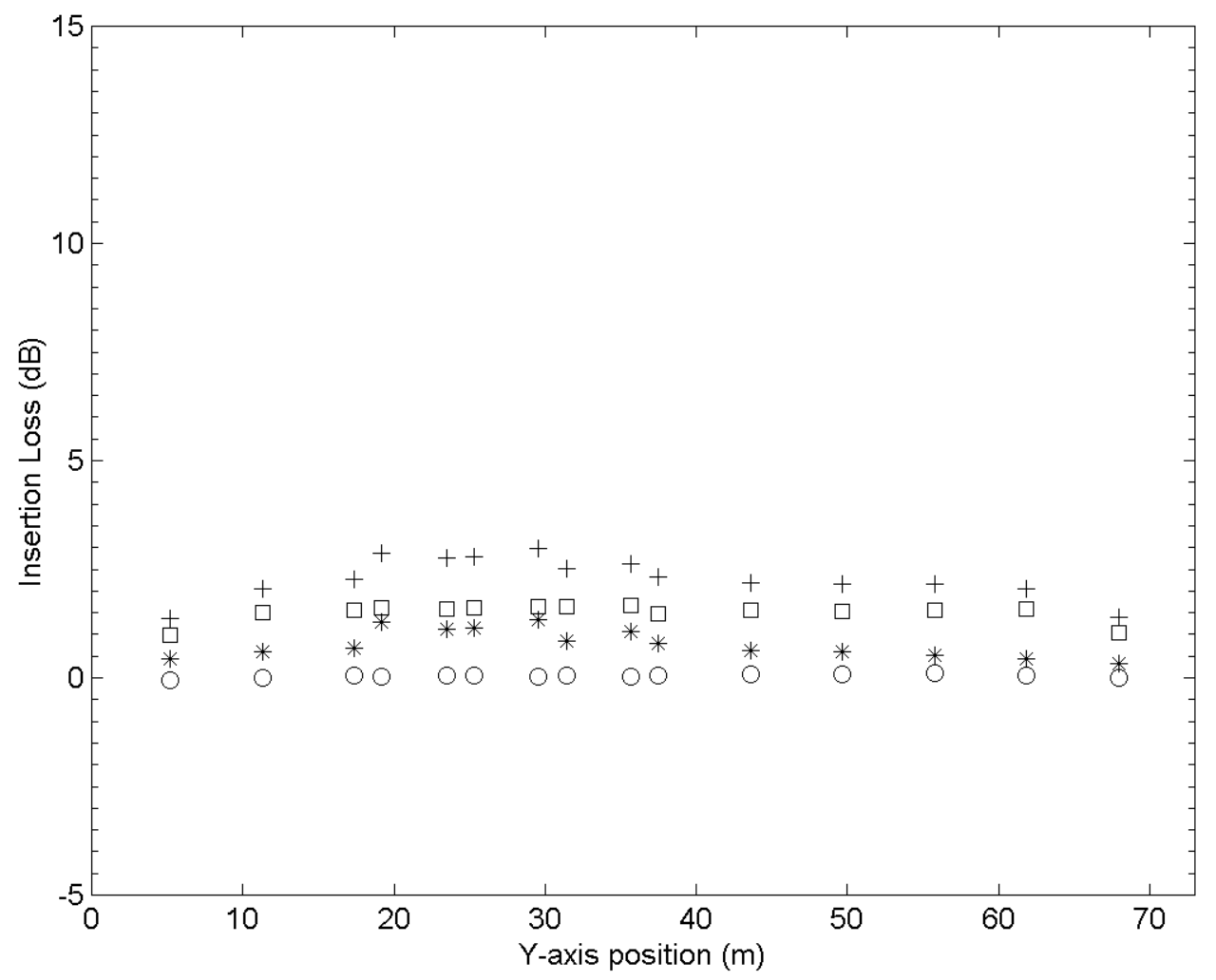

Figure 4.10: Insertion loss of all sources active as a function of window position. * insertion loss of absorbing canopy; $\mathrm{O}$ insertion loss of absorbing barriers; insertion loss of absorbing barriers; + insertion loss of all modifications.

\subsubsection{Verification of the Direct Field}

In the previous two sections, results are presented which indicate the importance of the direct field. To confirm the importance of the direct field two additional model configurations were tested. The models consisted only of the toll plaza ground, the vehicle sound sources, and the window receiver locations. The first model utilized semi-truck sources as described in the previous sections while the second model used passenger car sources which will be explained in this section. In MATLAB one lane of sources were activated at a time. The overall level and the insertion loss at the receiver locations of the lane with active sources, only, were analyzed. The verification models follow a strategy 
where the ground behaves as in the reference model but every other aspect of the lane near the sound source is perfectly absorbing with respect to sound.

One lane at a time semi-truck sources were activated, and the insertion loss results for the case with booths and canopy was compared to the case with no booths or canopy. Representative results are shown in Figures 4.11 and 4.12. Reference the Appendix for remaining results. The results were consistent across each lane, varying only a fraction of a $\mathrm{dB}$. The results confirmed the previous conclusions and indicate that the direct field accounts for all but 3 to $4 \mathrm{~dB}$. While this is a noticeable change, the noise control potential is limited to these reductions and no greater insertion loss can be achieved without controlling the source. 


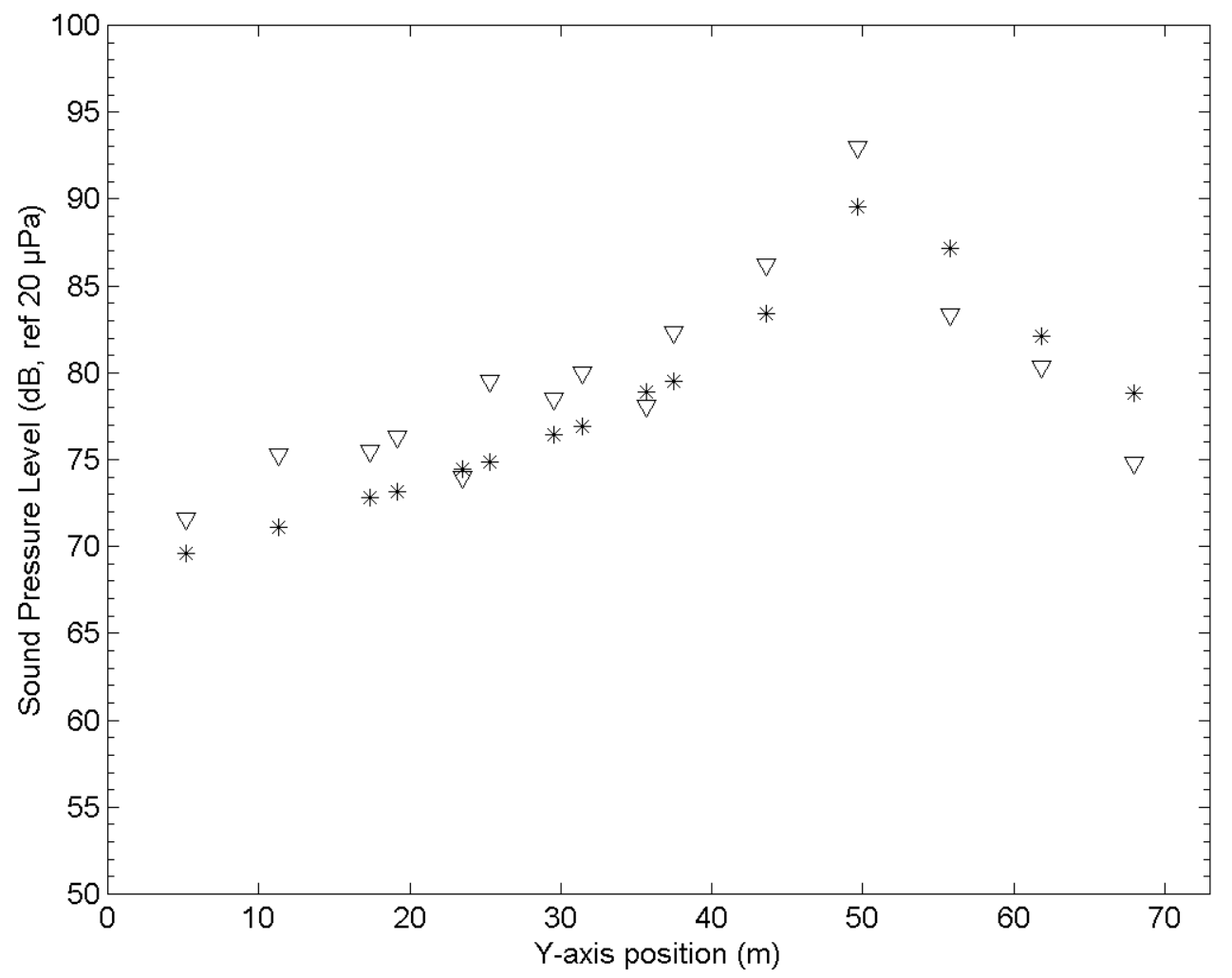

Figure 4.11: Overall level for all sources in the fourth lane from the north, semi-truck sources. $\nabla$ level of existing toll plaza; $*$ level with of direct field validation model. 


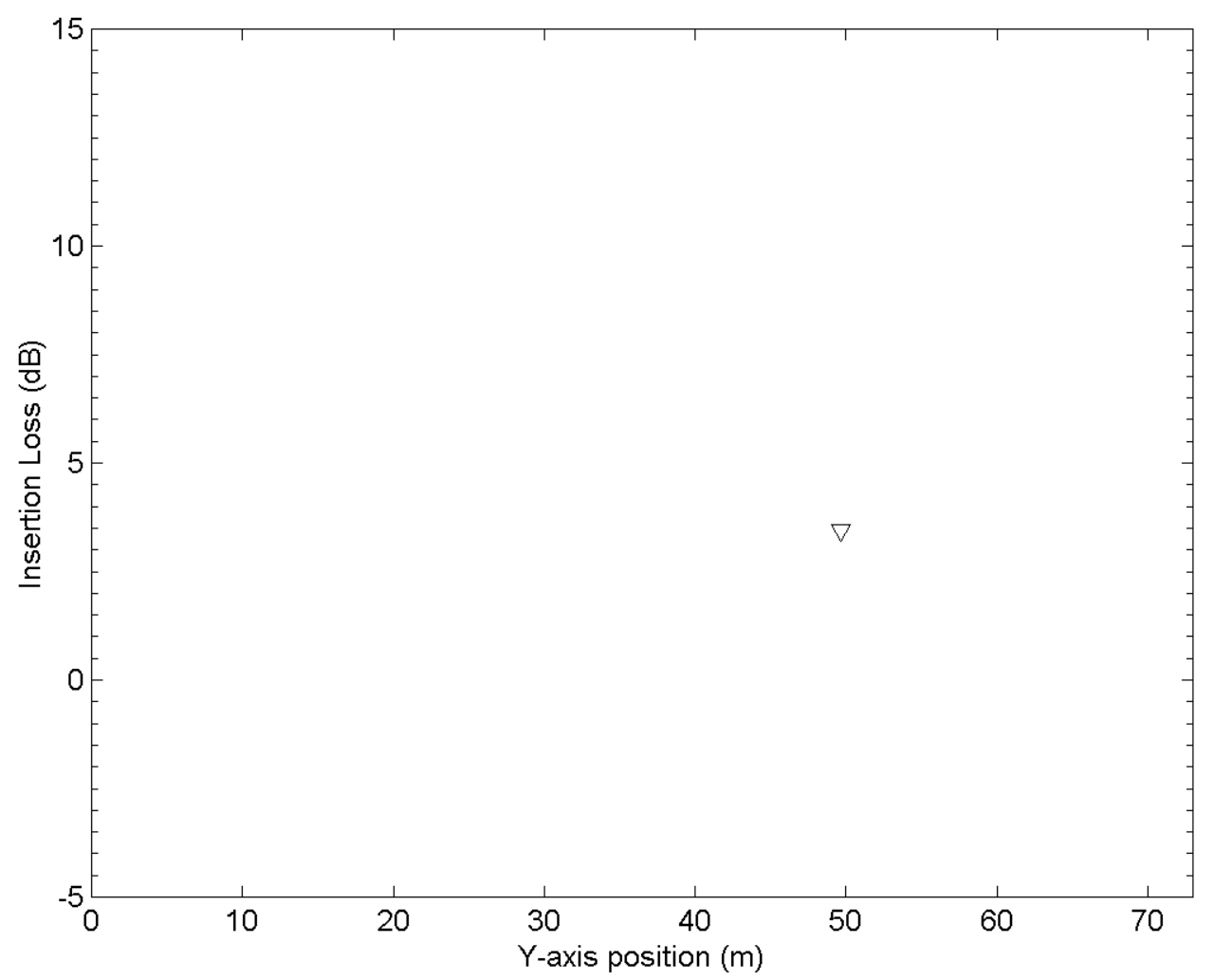

Figure 4.12: Insertion loss for all sources in the fourth lane from the north, semi-truck sources. $\nabla$ total absorbing elements as specified in the direct field validation model.

To ensure that the insertion loss results obtained for semi-truck sources extend to passenger vehicles, the lane source study was repeated for automobile sources. Every aspect of the previous source model remained the same except the source height and spectrum. Like the semi-truck source models, the automobile source models were constructed with reference to the TNM source model data. TNM models each automobile with two sources, one at ground level and the other at 1.52 meters $\left(5^{\prime}\right)$. Like the semi-truck source model, the lower source was moved to 0.15 meters $\left(0.5^{\prime}\right)$ above ground level to prevent anomalous transmissions through the ground of the model. To model an idling passenger car, TMN data for automobiles on average pavement traveling at $16.09 \mathrm{kph}$ (10 mph) under cruise throttle was used. To model an accelerating passenger car, TMN data for 
automobiles on average pavement traveling at $32.19 \mathrm{kph}(20 \mathrm{mph})$ under full throttle was used. The parameter selections were based upon the available options closest to the actual conditions. Within MATLAB, the TNM one third octave band sound pressure level data was converted to octave band sound power level data. The source values used in RAYNOISE are presented in Table 4.3.

\begin{tabular}{|l|l|l|l|l|l|l|l|l|}
\hline $\begin{array}{l}\text { Octave Band } \\
\text { Center } \\
\text { Frequency } \\
\text { (Hz) }\end{array}$ & $\mathbf{6 3}$ & $\mathbf{1 2 5}$ & $\mathbf{2 5 0}$ & $\mathbf{5 0 0}$ & $\mathbf{1 0 0 0}$ & $\mathbf{2 0 0 0}$ & $\mathbf{4 0 0 0}$ & $\mathbf{8 0 0 0}$ \\
\hline $\begin{array}{l}\text { Idle Low } \\
\text { Source (dB) }\end{array}$ & 55.4902 & 60.5765 & 62.2934 & 65.2934 & 67.8664 & 67.9038 & 62.5070 & 54.8704 \\
\hline $\begin{array}{l}\text { Idle High } \\
\text { Source (dB) }\end{array}$ & 53.3641 & 58.4504 & 60.1673 & 63.1673 & 63.7646 & 55.1966 & 45.6051 & 37.9684 \\
\hline $\begin{array}{l}\text { Acceleration } \\
\text { Low Source } \\
\text { (dB) }\end{array}$ & 70.0928 & 74.9085 & 77.2934 & 81.0545 & 84.5933 & 83.9038 & 77.5070 & 70.4689 \\
\hline $\begin{array}{l}\text { Acceleration } \\
\text { High Source } \\
\text { (dB) }\end{array}$ & 67.9668 & 72.7824 & 75.1673 & 78.9285 & 80.3639 & 71.1966 & 60.6051 & 53.5669 \\
\hline
\end{tabular}

Table 4.3: The automobile source spectrum used in the direct field verification model.

One lane at a time car sources were activated, and the insertion loss results for the case with booths and canopy was compared to the case with no booths or canopy. Representative results are shown in Figures 4.13 and 4.14. Reference the Appendix for remaining results. The results confirm the previous conclusions and indicate that the direct field accounts for all but 2.5 to $4 \mathrm{~dB}$ which is very similar to the results obtained for semitruck sources. As mentioned previously, passenger cars produce a much lower level than semi-trucks and as a result do not produce that situations of greatest concern of this research project. 


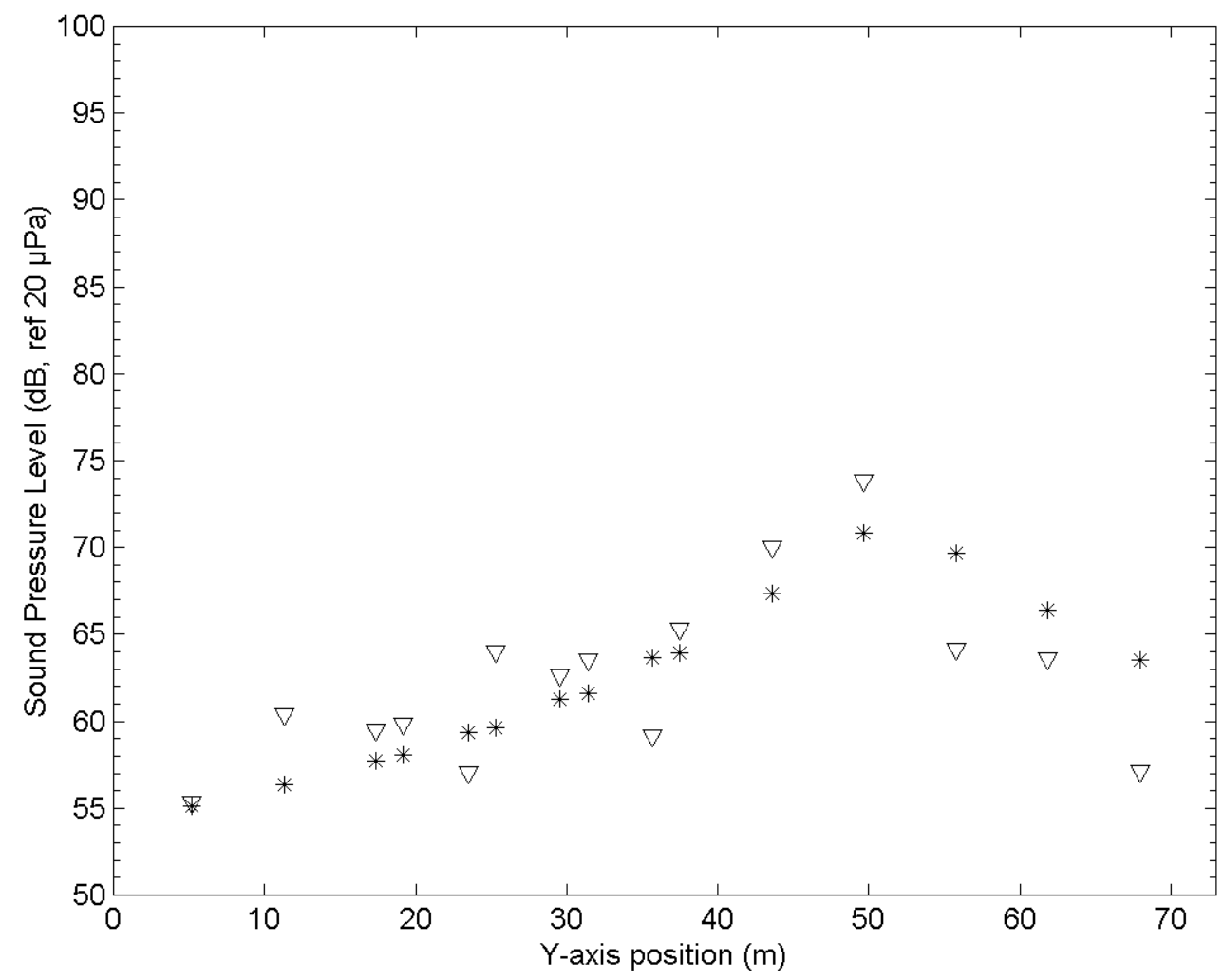

Figure 4.13: Overall level for all sources in the fourth lane from the north, car sources. $\nabla$ level of existing toll plaza; * level with of direct field validation model. 


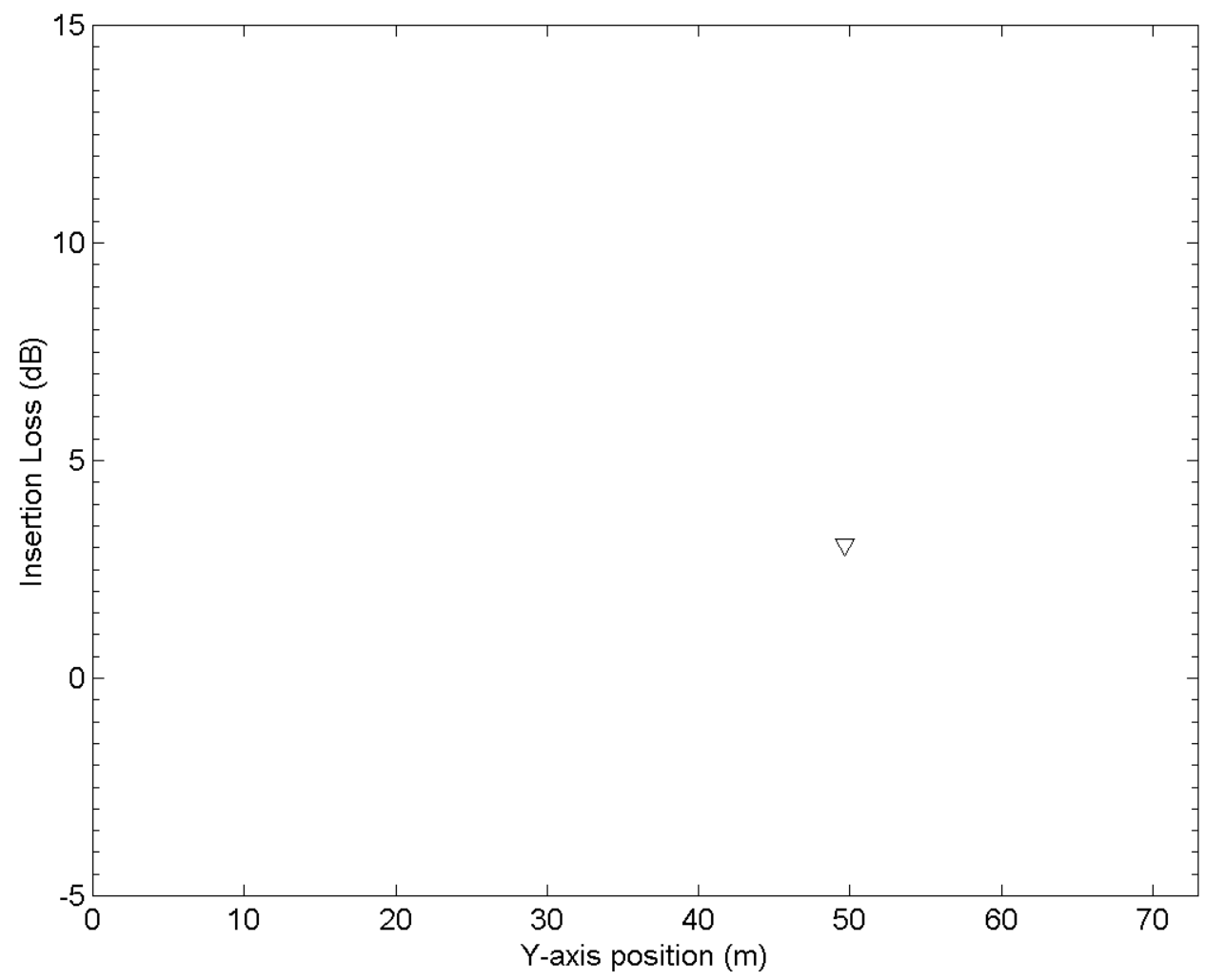

Figure 4.14: Insertion loss for all sources in the fourth lane from the north, car sources. $\nabla$ total absorbing elements as specified in the direct field validation model.

\section{$\underline{4.4 \text { Conclusions }}$}

The models predict that realistic implementation of passive sound absorbing techniques will achieve peak reductions of $3 \mathrm{~dB}$. Furthermore, the results suggest that the direct field accounts for all but 3 to $4 \mathrm{~dB}$ of the sound pressure level experienced by the tollbooth operators. While a reduction of $3 \mathrm{~dB}$ is perceivable, it is not substantial. In borderline circumstances, $3 \mathrm{~dB}$ may be enough to comply with noise exposure regulations or improve the environment. However, to reduce the noise level significantly, the noise control solution must address the direct field. ANC solutions that are intended to deal with 
the direct field and an evaluation of their effect upon speech intelligibility will be discussed in the next chapter.

The modeling approach described in Chapter 3 and implemented in this chapter does not account for coherent reflections that cause reverberation and resonance. Due to the statistically random nature of vehicle noise, it is a reasonable approximation to use a source model assuming incoherent waves where phase is neglected. If resonance conditions occur at the toll plaza, placement of absorbing material may have a more dramatic effect than predicted by the model. 


\section{ACTIVE NOISE CONTROL SYSTEMS}

\section{$\underline{5.1 \text { Introduction }}$}

Active Noise Control (ANC) systems were investigated to address both improvements in speech intelligibility and noise reduction. The potential of active noise control systems to reduce low frequency sounds within partially open enclosures has been demonstrated in previous studies by Waters (1988) and Waters and Bernhard (1989). The impact of such systems on speech intelligibility, however, is not well understood. Improvement may be possible if dominant low frequency sounds in traffic noise masks speech signals and reduces intelligibility.

The investigation included three ANC headsets and a prototype ANC system designed to create a local region of attenuation. The systems utilize single channel feedback algorithms. Single channel feedback ANC system embodiments generally display strong low frequency but are not adaptive. For the purposes of evaluating the speech intelligibility of the ANC systems, the Speech Intelligibility Index (SII) was used. The SII is a weighted signal to noise ratio calculation that takes into account masking and overloading effects. Furthermore, the SII is appropriately sophisticated to achieve results highly correlated to actual communication situations. As long as an ANC system behaves linearly in the tested frequency bands, the effects of the ANC system can be accounted for simply from its transfer function. The physical attenuation of each device for noise reduction purposes is also of interest. The system transfer functions were used to evaluate the noise attenuation performance of the ANC systems. 


\section{$\underline{5.2 \text { ANSI SII }}$}

An overview of the calculation procedures with emphasis on the fundamental equations will be presented in this section. Further details may be found in the ANSI S3.51997 standard (American National Standards Institute,1998).

\subsubsection{Calculation Method}

The standard specifies four calculation methods for the SII (American National Standards Institute,1998). Each method uses a different set of frequency bands. The methods from highest to lowest level of sophistication utilize the following frequency bands: 21 critical frequency bands, 18 one-third octave frequency bands, 17 critical frequency bands, and 6 octave frequency bands. Further information may be found in chapter 3.4 and 4 (American National Standards Institute,1998). The one-third octave frequency band method was used for this investigation. The one-third octave bands range from a center frequency of $160 \mathrm{~Hz}$ to $8000 \mathrm{~Hz}$.

\subsubsection{Input Data}

The next step of the SII calculation is the specification of input data. Three procedures are available to predict the SII accurately for different communication situations. The first procedure is for low reverberant environments where speech and noise are independent and can be measured. The second procedure is for reverberant environments where the speech signal causes echoes. Echoes are a form of speech distortion and contribute to the overall noise. The third procedure is for communication conditions that 
don't meet the necessary conditions for the first two procedures, namely conditions involving a device with unequal amplification to each ear. Information concerning the calculation methods for the input data, can be found in chapter 5 (American National Standards Institute,1998). The necessary conditions of the first procedure, where the input data are independent and measured, are satisfied at the toll plaza. Therefore the first procedure for calculation of the input data was selected.

The input data for the SII calculation are the equivalent speech spectrum level, the equivalent noise spectrum level, and the equivalent hearing spectrum threshold level. The speech spectrum level for the $\mathrm{i}^{\text {th }}$ frequency band, designated $E_{i}$ is the measured speech level for a $1 \mathrm{~Hz}$ wide frequency band at the center frequency of the $i^{\text {th }}$ frequency band. Suggested speech spectrum levels are included in the standard for normal, raised, loud, and shout speech vocal efforts. For situations where the speech is amplified or attenuated, an equivalent speech level, $E_{i}^{\prime}$, must be calculated from the speech spectrum levels and the insertion gain of the speech amplification system. The insertion gain for the $i^{\text {th }}$ frequency band, designated $G_{i}$, is the system transfer function at the center frequency of the $\mathrm{i}^{\text {th }}$ frequency band. The system transfer functions of interest for this study are the transfer functions between the spectrum density levels with the ANC systems and the spectrum density levels without the ANC systems. The equivalent speech spectrum level for the $i^{\text {th }}$ frequency band is

$$
E_{i}^{\prime}=E_{i}-20 * \operatorname{LOG}\left(d / d_{0}\right)+G_{i}
$$

where $d$ is the distance in meters form the talker's lips to the listener's ears and $d_{0}$ is the reference distance, 1 meter.

In a similar manner, the equivalent noise spectrum level for the $\mathrm{i}^{\text {th }}$ frequency band, $N_{i}^{\prime}$, is 


$$
N_{i}^{\prime}=N_{i}+G_{i}
$$

where $N_{i}$, the noise spectrum level, is the measured noise level for a $1 \mathrm{~Hz}$ wide frequency band at the center frequency of the $\mathrm{i}^{\text {th }}$ frequency band. The noise spectrum level is measured at the location of the listener's head.

The equivalent hearing threshold spectrum level for the $\mathrm{i}^{\text {th }}$ frequency band,

designated $T_{i}^{\prime}$, is the arithmetic average of the hearing threshold level for all listeners of interest. The hearing threshold level is defined according to standard audiometric practice as the pure tone threshold level minus the reference level. The threshold level is a measure of the deviation of a subject's hearing response from normal. Further details will be given in subsequent sections.

\subsubsection{Calculation Procedures}

The SII is calculated using the following steps. The procedures are outlined in chapter 4 and the physical description of the parameters is discussed in chapter 3 (American National Standards Institute,1998).

The first step is to calculate the self-speech masking spectrum level for the $i^{\text {th }}$ frequency band, $V_{i}$,

$$
V_{i}=E_{i}^{\prime}-24
$$

The self-speech masking spectrum is a measure of the masking content in the speech signal. The upward slope of speech masking is

$$
C_{i}=-80+0.6 *\left[B_{i}+10 * \operatorname{LOG}\left(F_{i}\right)-6.353\right]
$$

where $B_{i}$ is the larger of $N_{i}^{\prime}$ or $V_{i}$ for the $\mathrm{i}^{\text {th }}$ frequency band and $F_{i}$ is the center frequency for the $\mathrm{i}^{\text {th }}$ frequency band as given in Table 3 of the standard (American National Standards Institute,1998). The upward slope of speech masking accounts for the low frequency masking of higher frequency information. Accordingly, the most complicated parameter, 
the equivalent masking spectrum level, can be computed. The equivalent masking spectrum level accounts for the maskers. The equivalent masking spectrum level of the lowest frequency band is

$$
Z_{1}=B_{1}
$$

For all but the lowest frequency band, the equivalent masking spectrum level for the $\mathrm{i}^{\text {th }}$ frequency band is

$$
Z_{i}=10 * \operatorname{LOG}\left\{10^{0.1 * N_{i}^{\prime}}+\sum_{k=1}^{i-1} 10^{0.1 *\left[B_{k}+3.32 * C_{k} * L O G\right.}\left(0.89 * F_{i} / F_{k}\right)\right] .
$$

The equivalent internal noise spectrum level for the $\mathrm{i}^{\text {th }}$ frequency band, $X_{i}^{\prime}$, is

$$
X_{i}^{\prime}=X_{i}+T_{i}^{\prime}
$$

where $X_{i}$ is the reference internal noise spectrum level as given in Table 3 (American National Standards Institute,1998). The reference internal noise is the hypothetical external masking sound which would produce the reference hearing threshold previously mentioned. For convenience in audiometric testing, the reference hearing threshold is defined as $0 \mathrm{~dB}$ across all frequencies. However, the human ear does not exhibit a true hearing threshold of $0 \mathrm{~dB}$ referenced to 20 micro Pascals. The reference internal noise is used to correct the hearing threshold.

The equivalent disturbance spectrum level for the $\mathrm{i}^{\text {th }}$ frequency band, designated $D_{i}$, is the larger of $Z_{i}$ or $X_{i}^{\prime}$ for each of the frequency bands. Usually a higher speech level entails improved intelligibility. However, at high levels the speech signal can overload the ear. The level distortion factor for the for the $\mathrm{i}^{\text {th }}$ frequency band, $L_{i}$, is

$$
L_{i}=1-\left(E_{i}^{\prime}-U_{i}-10\right) / 160
$$

where $U_{i}$ is the standard speech spectrum level at normal vocal effort as given in Table 3 of the standard (American National Standards Institute,1998). The upper value of $L_{i}$ is limited to not exceed unity. 
The previous steps allow for the calculation of the band audibility function. For a given frequency band, the audibility function indicates the percentage of the speech signal in the band that is intelligible. It is a measure of the speech signal which is not masked or distorted for a given frequency band. The calculation of the band audibility function for the $\mathrm{i}^{\text {th }}$ frequency band is

$$
A_{i}=L_{i} *\left[\left(E_{i}^{\prime}-D_{i}+150\right) / 30\right]
$$

where the quantity in brackets is limited to values between 0 and 1 . The band importance function, $I_{i}$, is a measure of the percentage of information carried within a given frequency band. Therefore the SII is

$$
S=\sum_{i=1}^{n} I_{i} * A_{i}
$$

\subsubsection{Verification}

Procedures were coded to calculate the SII using the one-third octave band calculation method given the input data. A verification case for the code was used to establish that all of the calculation steps were coded correctly. Annex C.2 includes a partial calculation example for the one-third octave band method. This verification exposed an error with the parameter $C_{1}$ as published in Table C.2 of the standard (American National Standards Institute,1998). The calculations were checked a second time manually to confirm the typographical error. This partial check established reliable coding of the first few steps including the equivalent masking spectrum. Verification of the equivalent masking spectrum level is the only parameter where the calculation is rather involved. The coding of the remaining steps, none of which were difficult, was reviewed multiple times to ensure correctness. 


\section{$\underline{5.3 \text { Input Data Acquisition }}$}

As explained in the previous sections of this chapter, the three input data sets equivalent speech spectrum level, equivalent noise spectrum level, and equivalent hearing threshold spectrum level - are necessary for the calculation of the SII. To account for the effects of each headset configuration, the insertion gain due to the system was measured.

\subsubsection{Calculation of System Insertion Gain}

The insertion gain as specified in the standard is "the difference in decibels between the pure-tone sound pressure level at the eardrum with the amplification/attenuation device in place and the pure tone sound pressure level at the eardrum with the device removed" (American National Standards Institute,1998, chap 3.28). The insertion gain for each ANC device can be measured as the transfer function of the device.

To obtain the insertion gain spectra, a head and torso simulator with microphones at the ear locations was used. The head and torso simulator along with two high efficiency full range loudspeakers were placed in the reverberant test room at Ray W. Herrick

Laboratories. A photograph of this configuration is shown in Figure 5.1. The reverberation room was used to create a sound field of random incidence to minimize any directional effects of the headsets. This configuration provided the most challenging environment for noise control. Since the insertion gain, only, was calculated in the reverberation room, the necessary conditions for input data calculations are not violated.

The input to the loudspeakers and the output from the microphones in the head and torso simulator were connected to a professional grade computer sound card. A test signal was played by the computer through the loudspeakers, and simultaneously, the response at the microphones of the head and torso simulator was recorded. For each test signal, 
comparison of the response at the microphone with and without the headsets gives the insertion loss of the device. To create consistent headphone placement, procedures from ANSI S12.42-1995 for the fitting of circumaural hearing protectors were used (American National Standards Institute, 1995, chap. 9.2.1).

For the TechnoFirst external system, a series of measurements were made with a sound level meter to establish the position of the system with respect to the head for best performance. In the final configuration, the speakers of the external ANC system were placed 0.08 meters from the microphones of the head and torso simulator. Note that the error microphones for the system are attached to the eye glasses. The system configuration is displayed in Figure 5.2. To begin the test a calibrated tone generator was placed on each of the microphones and the response was recorded to establish a calibration signal for the system. Similarly, the noise floor of the test equipment was recorded. 


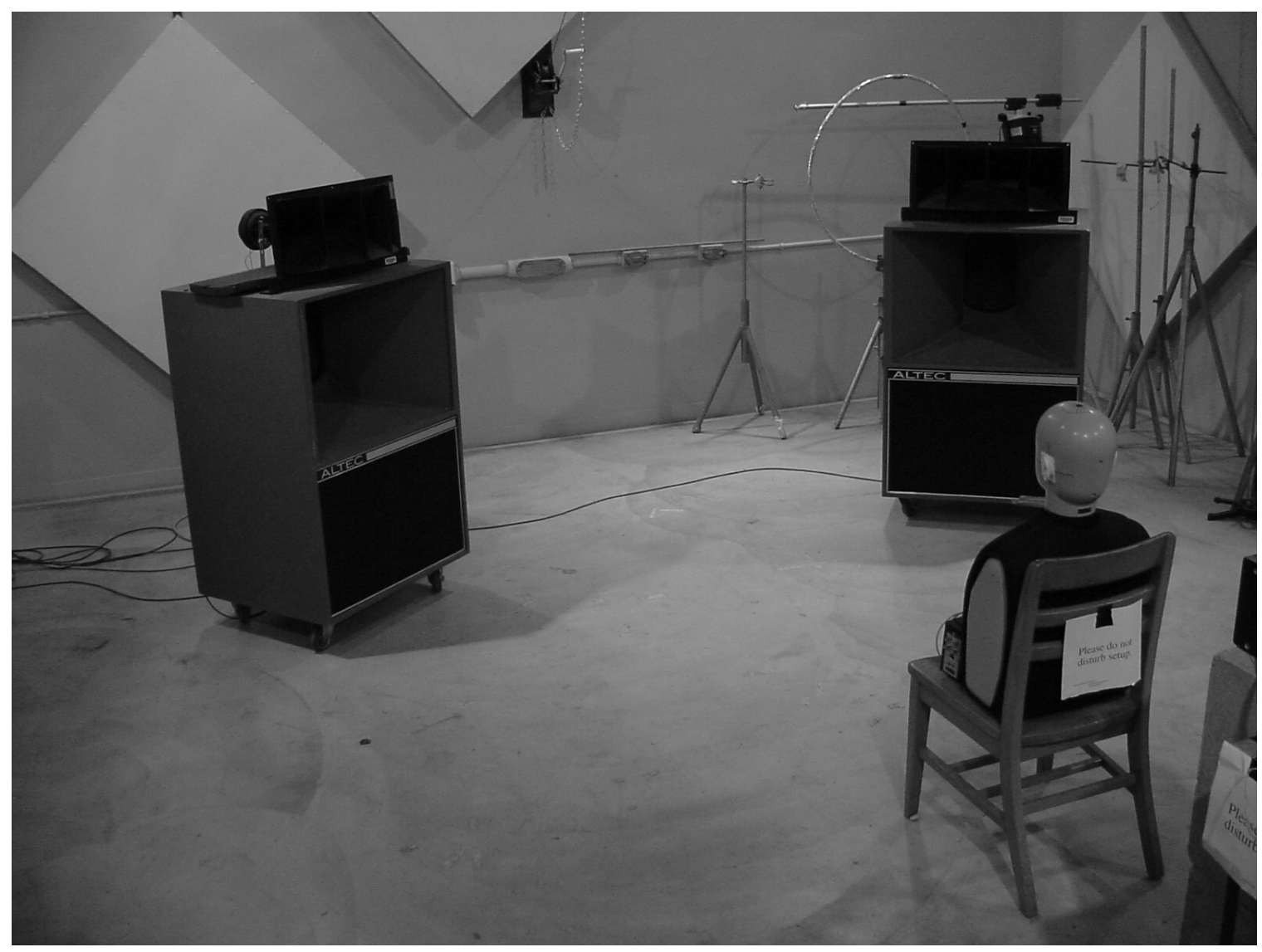

Figure 5.1: Photograph of the test configuration to obtain ANC system transfer functions. 


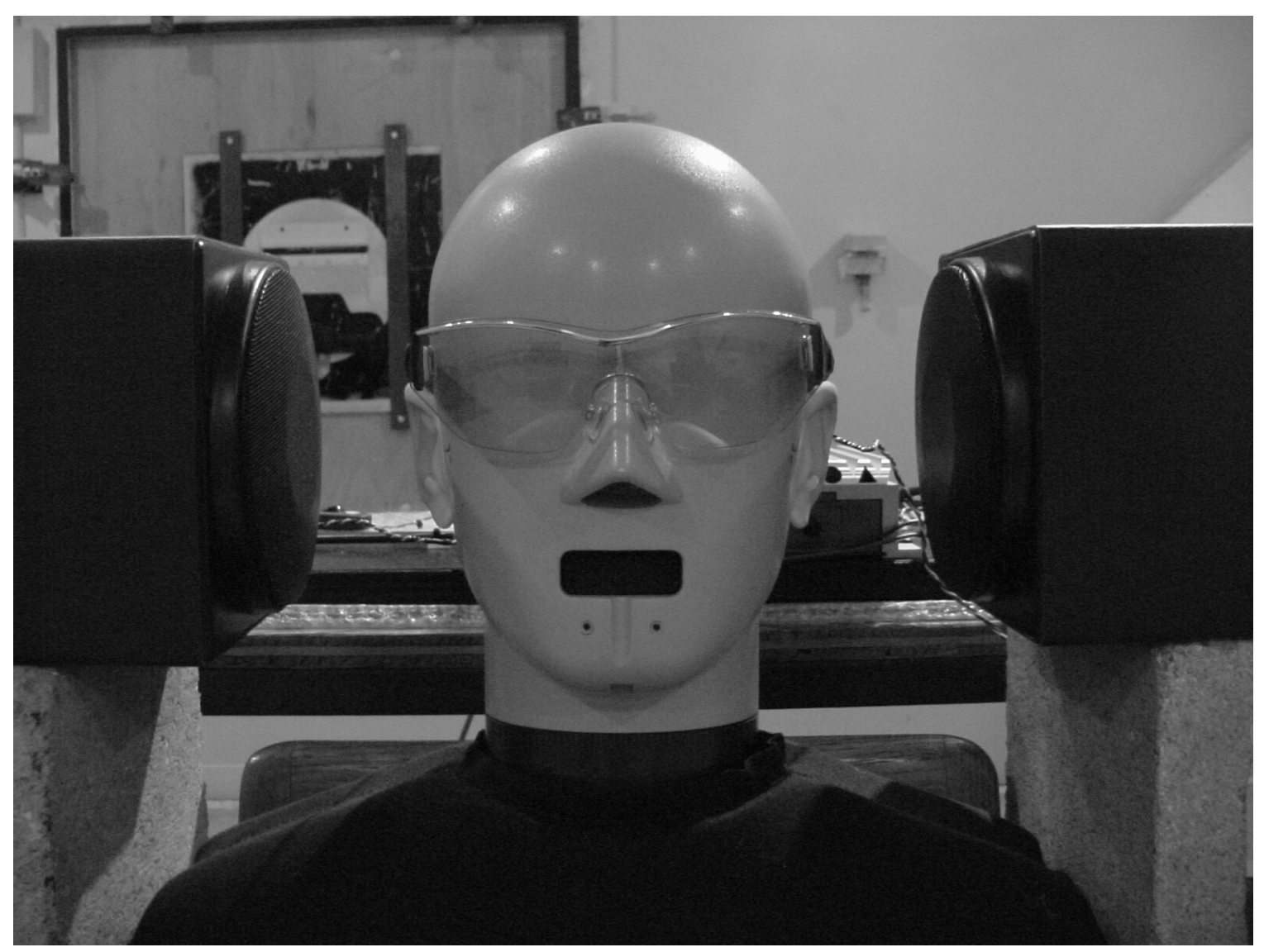

Figure 5.2: $\quad$ Location of the TechnoFirst ANC system with respect to the head and torso simulator.

\subsubsection{Insertion Gain Test Signals}

Three different 10 minute long test signals were created for calculation of the insertion gain. The first test signal was traffic noise. On November 21, 2000 approximately one hour of traffic noise was recorded at the Portage Barrier Toll Plaza. A single microphone was centered near the rear doorway of the $3^{\text {rd }}$ booth to the north and positioned approximately 1.22 meters (4') above the floor. The microphone protruded approximately 0.08 meters (3") from the rear of the booth to capture the sound of traffic traveling on the lane to the south. At the rear of the tollbooth, vocal conversation levels were minimal. The lane in which traffic noise was recorded served primarily semi-truck vehicles. 
The monaural recording was made with a portable digital audio tape (DAT) machine and matching, scientific quality microphone and power supply. The sampling frequency of the recording was $44,100 \mathrm{~Hz}$ with 16 bits of amplitude resolution. The level was calibrated at the beginning and the end of the recording. The recording level was adjusted prior to recording to be a maximum without clipping. The time domain data captured on the DAT recorder was digitally transferred to the test computer through the professional grade sound card. Due to a traffic accident on a distant section of the toll road, there were periods of intermittent traffic flow at the toll plaza during the recording. To create sounds from a typical traffic pattern, two recorded sections were spliced to make a 10 minute wave file.

The second test signal was random noise. The output of a random noise generator was connected to the professional grade sound card. A 10 minute wave file was collected with a sampling frequency of $44,100 \mathrm{~Hz}$ and an amplitude resolution of 16 bits.

The third test signal was speech. The lengthy monologue on track 6 of the Stereophile Test CD contained male speech that was recorded with many different, quality microphones (Holt, 1990). This track was partially repeated once to create a test signal 10 minutes in length.

\subsubsection{Processing Recorded Time Domain Data}

MATLAB code was used to calculate the insertion gain for each device in terms of one-third octave frequency bands. As discussed previously, the response signals recorded at the microphones of the head and torso simulator along with a calibration tone and noise floor were saved as wave files. MATLAB was used to load the calibration wave file, calculate a power spectral density via a Fourier transform, and calibrate the level of the pure tone. The remaining wave files were loaded into MATLAB and the data was converted to a calibrated power spectral density. The calibrated frequency data was then converted using 
one-third octave band filters generated in MATLAB. The frequency response of the onethird octave band filter is shown in Figure 5.3. The output of the one-third octave band filters was converted to spectrum density levels as specified by the ANSI standard (American National Standards Institute, 1998). The system transfer function for each device configuration was then calculated by subtracting the spectrum density level data for the signal without any ANC device from the spectrum density level at the head and torso simulator with the device. From the insertion gain data, the input data were calculated as described in the following sections. The signal to noise ratio was high for all of the insertion gain measurements; in the frequency range of interest the noise floor was at least $15 \mathrm{~dB}$ below the signal level and often $40 \mathrm{~dB}$ below the signal level.

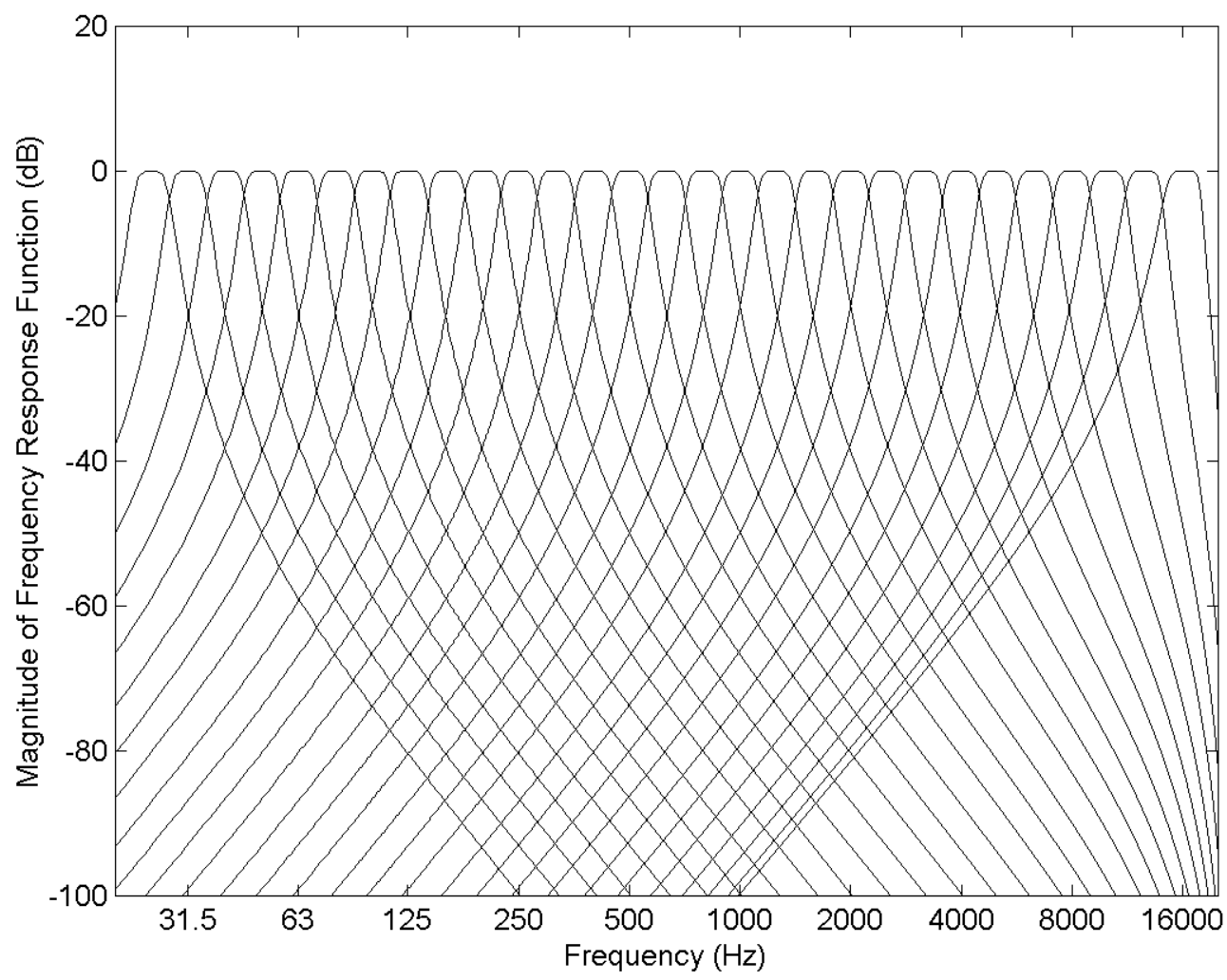

Figure 5.3: Frequency response of the one-third octave band filters. 


\subsubsection{Calculation of the Equivalent Speech Spectrum Level}

The equivalent speech spectrum levels were calculated using the standard speech spectrum levels included in the ANSI document (American National Standards Institute, 1998). For SII calculations, it is recommended that the standard speech spectrum levels are used unless data is available for a sufficient number of talkers sampled over a sufficiently long duration. On average, it was believed that the distance between the speaker and listener will be between 0.75 to 1 meter at the tollbooths. The shorter distance is representative of the talker and listener leaning towards one another. The standard speech spectrum levels are given for four vocal efforts: normal, raised, loud, and shout.

\subsubsection{Calculation of the Equivalent Noise Spectrum Level}

Three equivalent noise spectrum level sets were calculated using traffic noise recordings from the Portage Barrier Toll plaza. The first set was indicative of typical traffic sounds from semi-trucks including deceleration, idle, and acceleration vehicle conditions. The traffic noise calibration wave file and the 10 minute traffic noise wave file created for the insertion gain calculation were imported into MATLAB. In MATLAB the calibrated traffic noise file was filtered by one-third octave band filters. The noise spectrum level was calculated from the one-third octave band output.

The second equivalent noise spectrum level set represented idling semi-trucks. Communication at the toll plaza would primarily occur while the truck was stationary and idling. Therefore, idling semi-truck noise would represent a realistic masking sound for the toll plaza. The audio file of typical semi-truck sounds was reviewed multiple times and edited. The result was over 5 minutes of various semi-truck idling sounds. In MATLAB 
this calibrated traffic noise file was filtered by one-third octave band filters. The noise spectrum level was calculated from the one-third octave band output.

The final equivalent noise spectrum level set represents idling passenger cars. The TNM source data used for RAYNOISE predictions was reviewed to establish a transfer function between idling semi-trucks and idling cars. This transfer function was used to convert the noise spectrum level for idling semi-trucks to a noise spectrum level for idling cars.

\subsubsection{Calculation Of Equivalent Hearing Threshold Spectrum Level}

The standard specifies a reference hearing threshold spectrum level of $0 \mathrm{~dB}$ across all frequencies. This is the hearing threshold level spectrum for otologically normal listeners between the ages of 18 to 30 . This reference level was used instead of measuring and averaging the hearing threshold spectrum level of tollbooth workers.

\subsection{Analysis and Results}

Three ANC headsets systems - the Noise Buster Extreme, the ProActive 1500, and the ProActive 3500 - and one external ANC device designed to be mounted to the tollbooth to create a local region of attenuation were tested. Photographs of the ANC systems are shown in Figures 5.4 through 5.7. The three ANC headset systems were manufactured by NCT Inc. The Noise Buster Extreme is a partially open ear device built for listening to music through a portable entertainment system. Open ear headsets do not suppress ambient noise through passive approaches. The ProActve 1500 is an open ear designed for industrial applications where the ear pads rest upon the user's ears. The ProActive 3500 is a closed ear circumaural industrial design where the ear cups seal against the user's head. The 
closed ear design offers significant passive attenuation which provides further noise attenuation at high frequency. The external ANC system is a prototype built by TechnoFirst which is researching a similar configuration to be installed in airplane seats. While the external ANC system will have less ANC attenuation due to physical limitations, it does not contain passive components which may impede communication. The lack of passive components additionally is often better for user comfort.

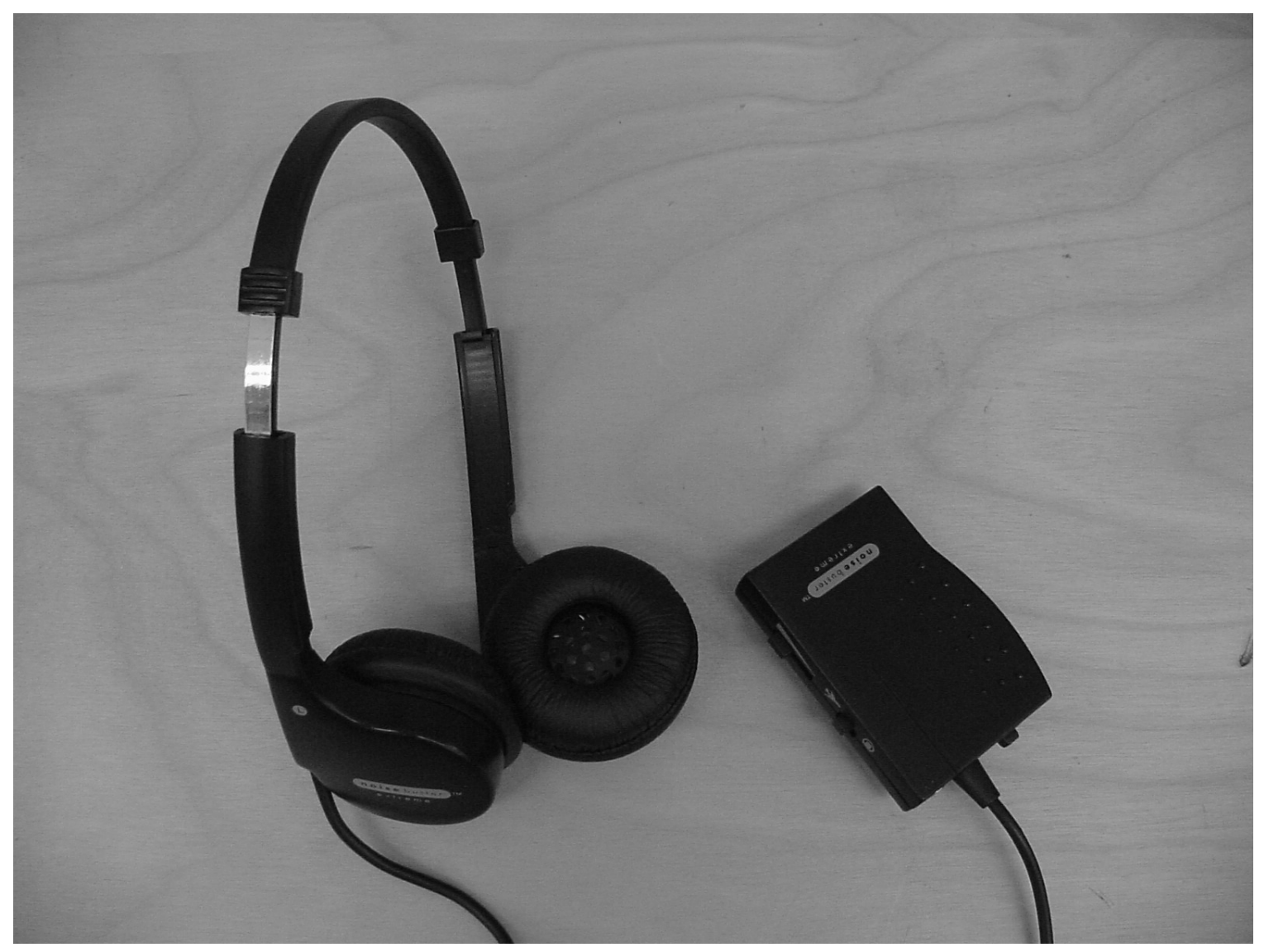

Figure 5.4: Photo of the NCT Noise Buster Extreme ANC Headsets. 


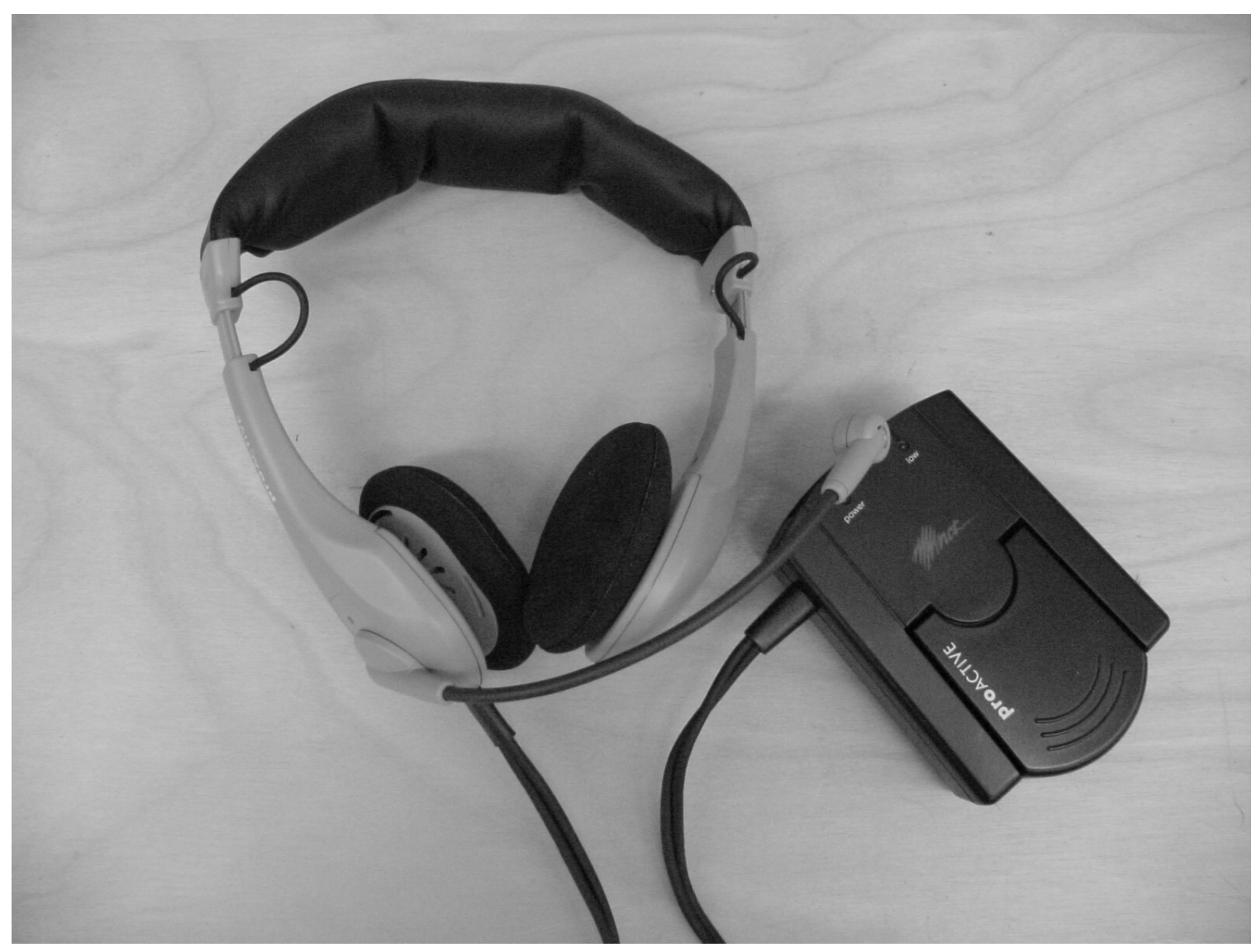

Figure 5.5: Photo of the NCT ProActive 1500 ANC Headsets. 


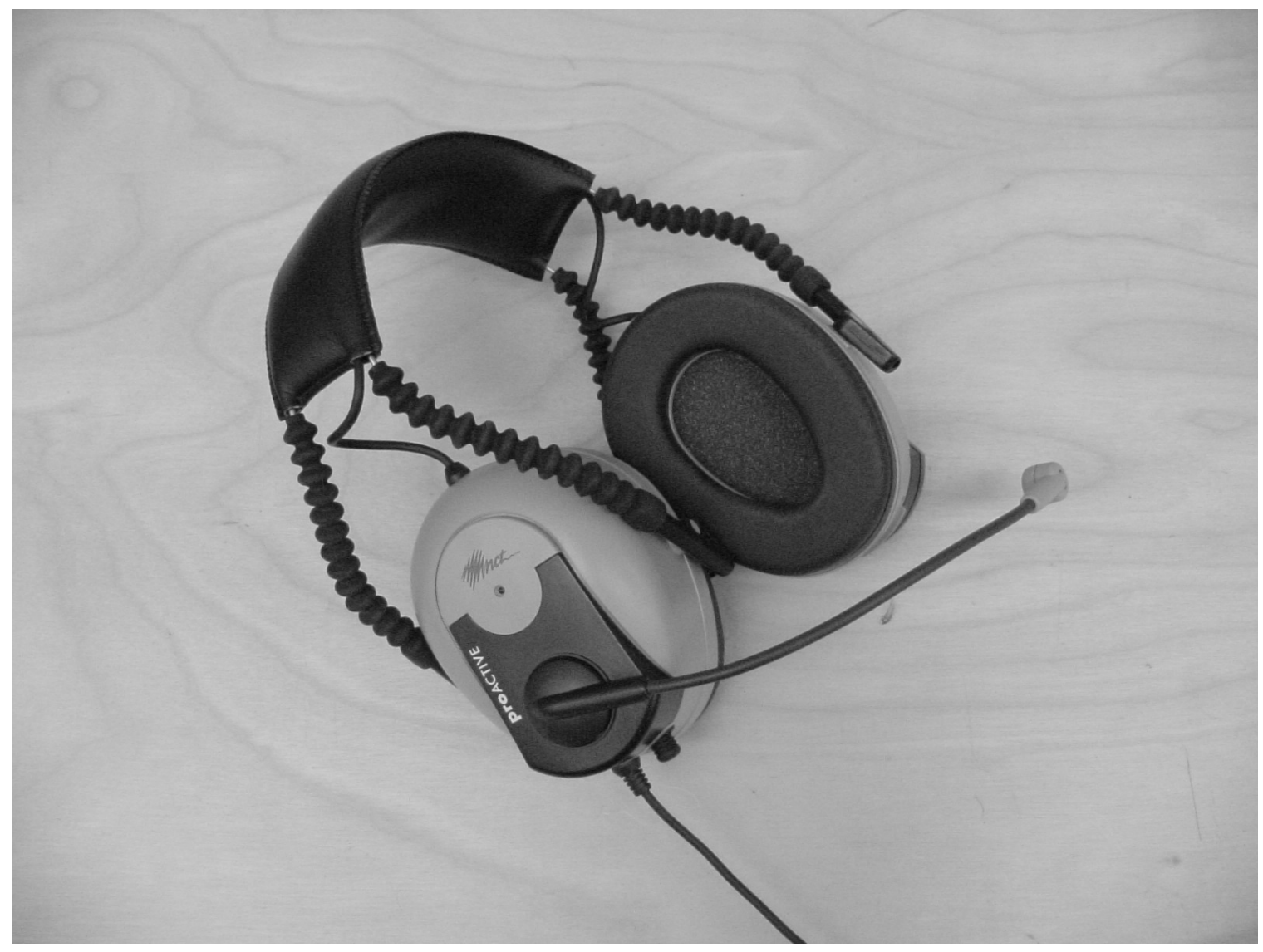

Figure 5.6: Photo of the NCT ProActive 3500 ANC Headsets. 


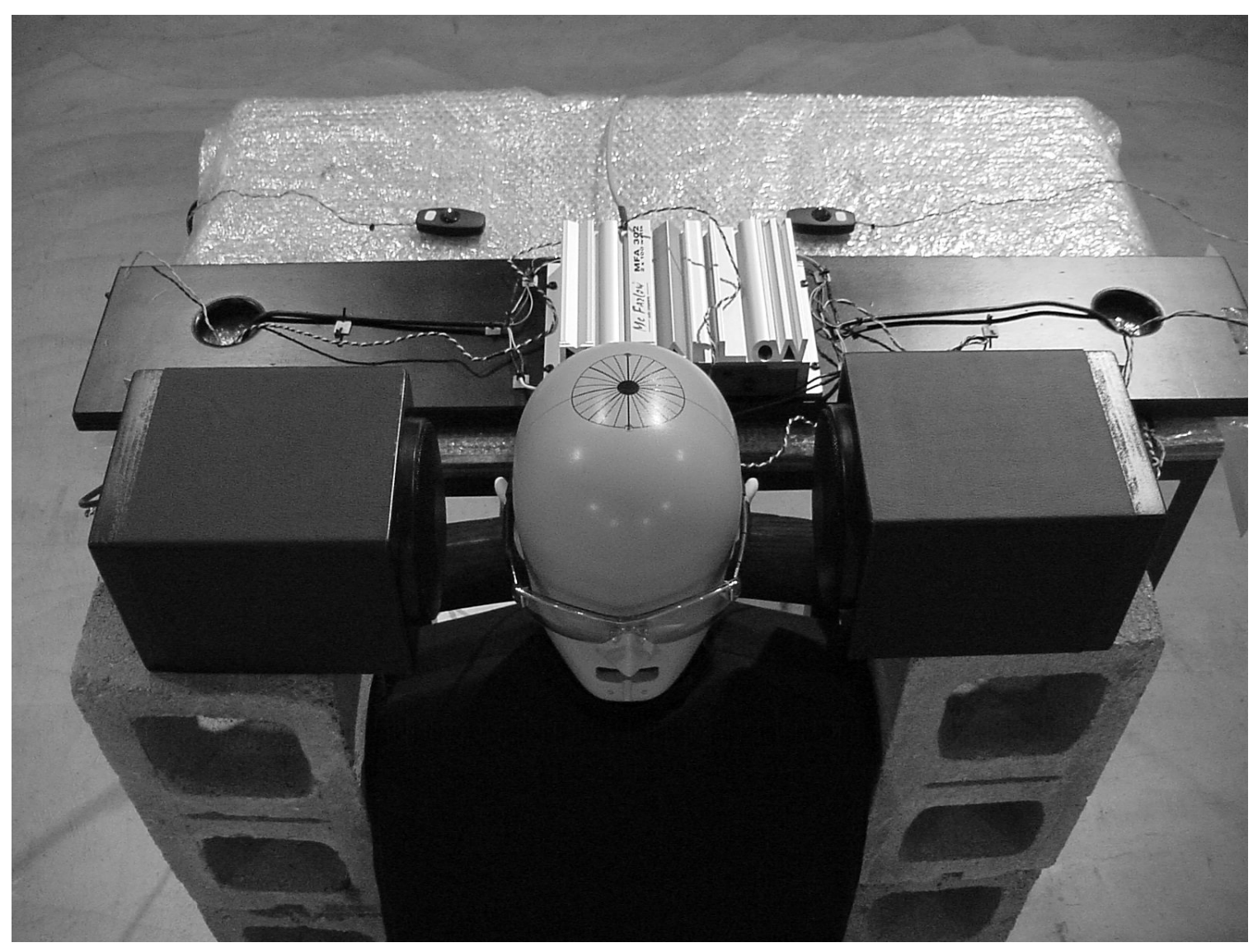

Figure 5.7: Photo of the TechnoFirst Prototype External ANC System and the head and torso simulator.

\subsubsection{Physical Attenuation Performance of ANC Systems}

As described in section 5.3.1.2, MATLAB code was used to calculate the system transfer function for each ANC system in terms of one-third octave bands. The results are displayed graphically in Figures 5.8 through 5.11. The insertion loss performance of the tested systems is relatively immune to the type of input signal used. The systems behave linearly and therefore meet the necessary conditions of the ANSI SII calculation procedures.

The NCT Noise Buster Extreme headset and the NCT ProActive 1500 exhibit similar performance characteristics. Both have a maximum low frequency attenuation 
greater than $10 \mathrm{~dB}$ at $250 \mathrm{~Hz}$. Both devices have amplification in the $50 \mathrm{~Hz}$ frequency region and the $1000 \mathrm{~Hz}$ frequency region. The Noise Buster Extreme has higher frequency attenuation than the ProActive 1500, which is a result of passive components.

The NCT ProActive 3500 amplifies the low frequencies levels near $40 \mathrm{~Hz}$. However, the ProActive 3500 exhibits significant attenuation performance for the rest of the frequency range. Much of the low frequency reduction is in excess of $20 \mathrm{~dB}$ with a maximum of $40 \mathrm{~dB}$ at $3000 \mathrm{~Hz}$.

The prototype external ANC system from TechnoFirst produced $10 \mathrm{~dB}$ of fairly narrow band reduction at $200 \mathrm{~Hz}$. Low frequencies and middle frequencies were amplified. The TechnoFirst system did not display the passive high frequency noise reduction of the headsets. In addition, some of the high frequency sounds were amplified due to near reflections caused by the physical constructs of the TechnoFirst system. 


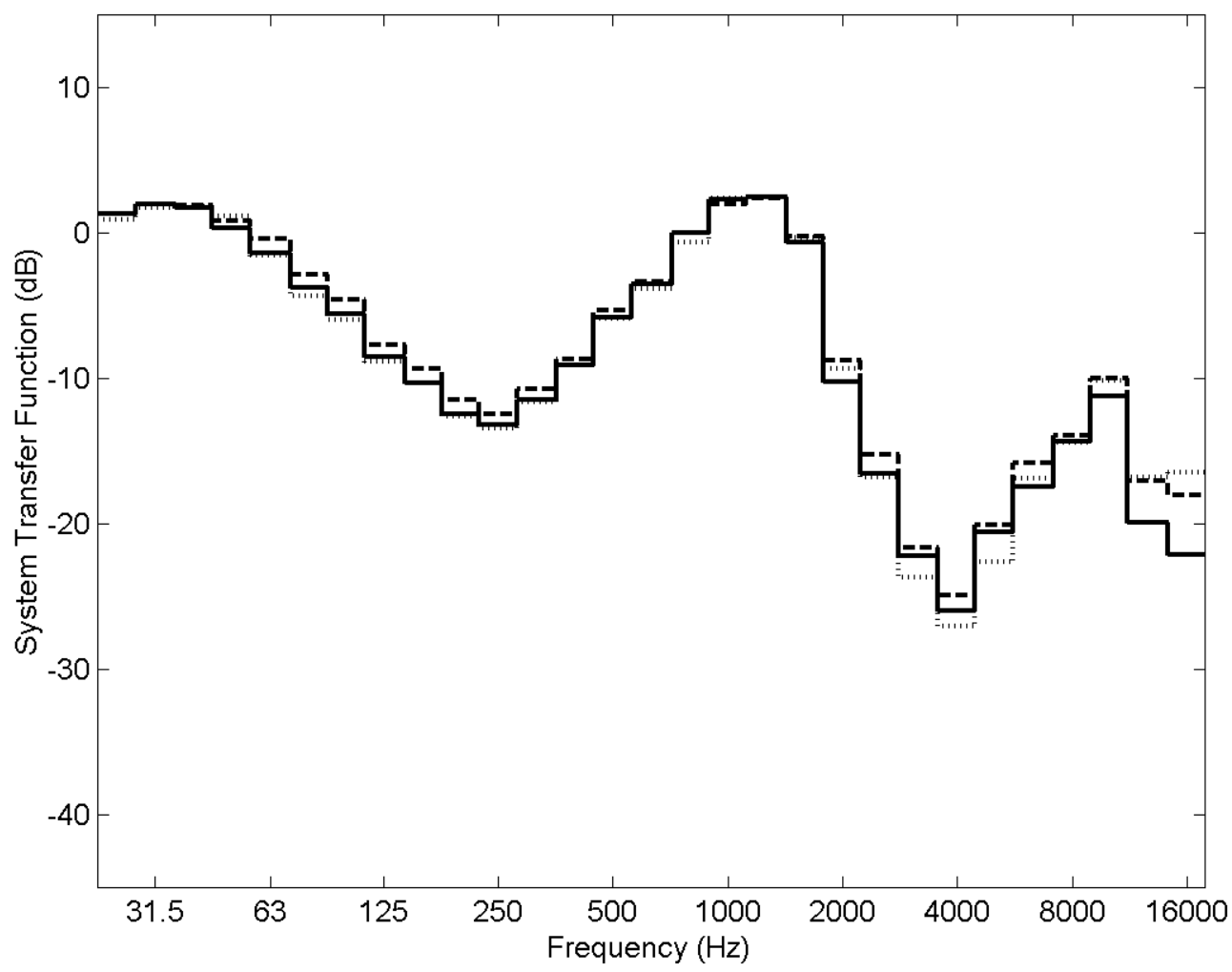

Figure 5.8: Transfer function for the NCT Noise Buster Extreme ANC headset, right ear with the active noise control setting at the maximum position in terms of one-third octave bands for different signals. - random noise signal; .. speech signal; -- traffic signal. 


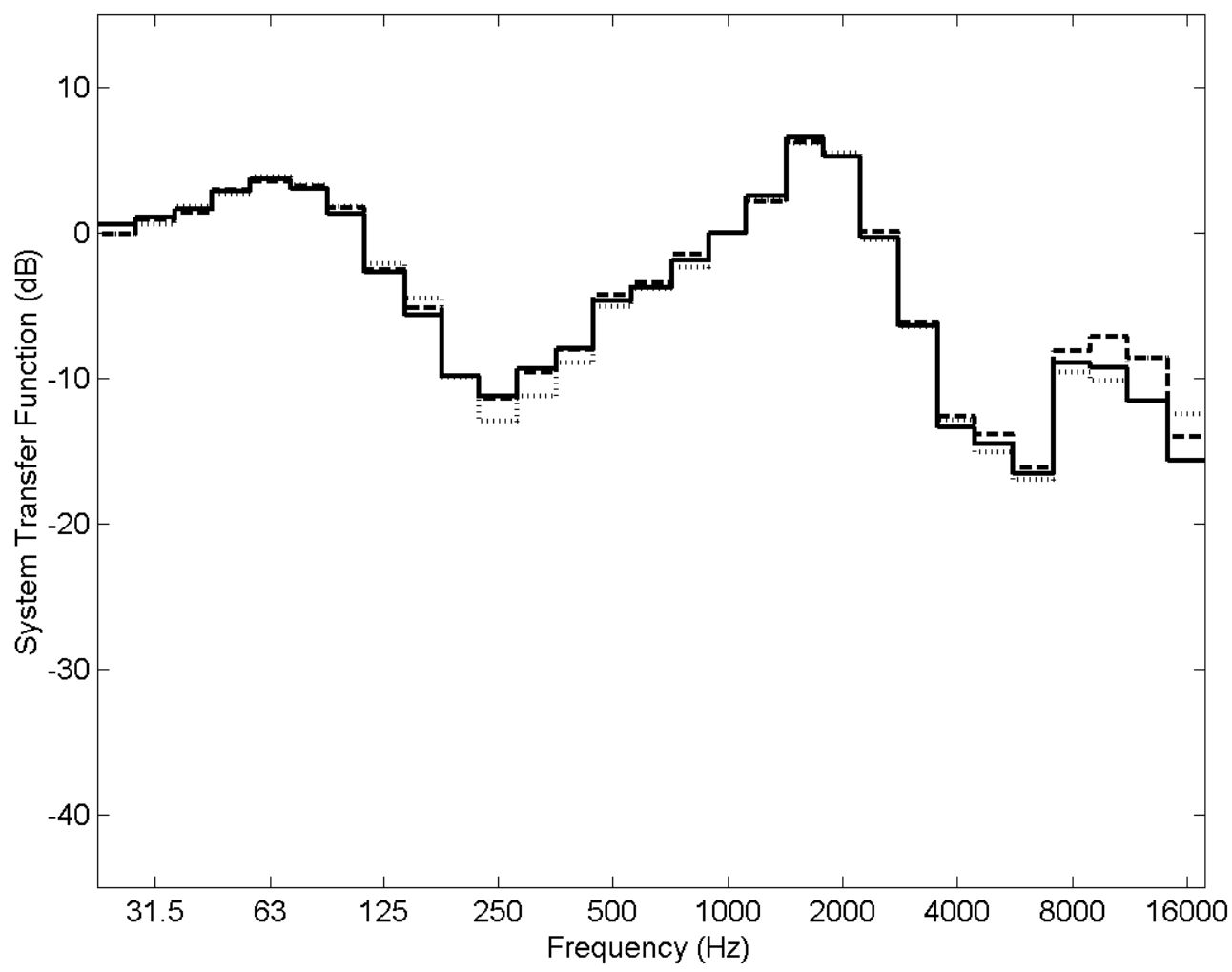

Figure 5.9: Transfer function for the NCT ProActive 1500 ANC headset, right ear in terms of one-third octave bands for different signals. - random noise signal; .. speech signal; -- traffic signal. 


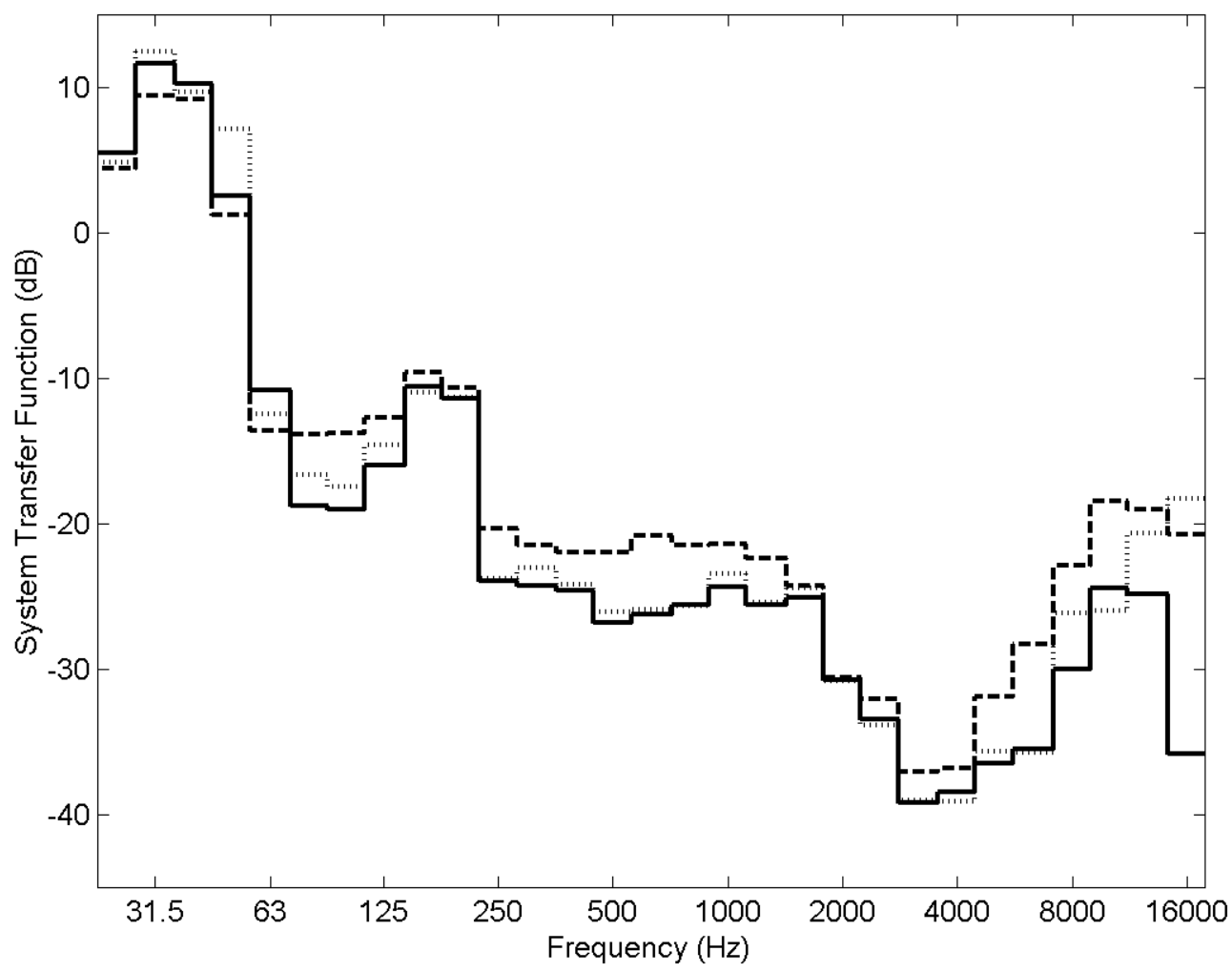

Figure 5.10: Transfer function for the NCT ProActive 3500 ANC headset, right ear in terms of one-third octave bands for different signals. - random noise signal; .. speech signal; -- traffic signal. 


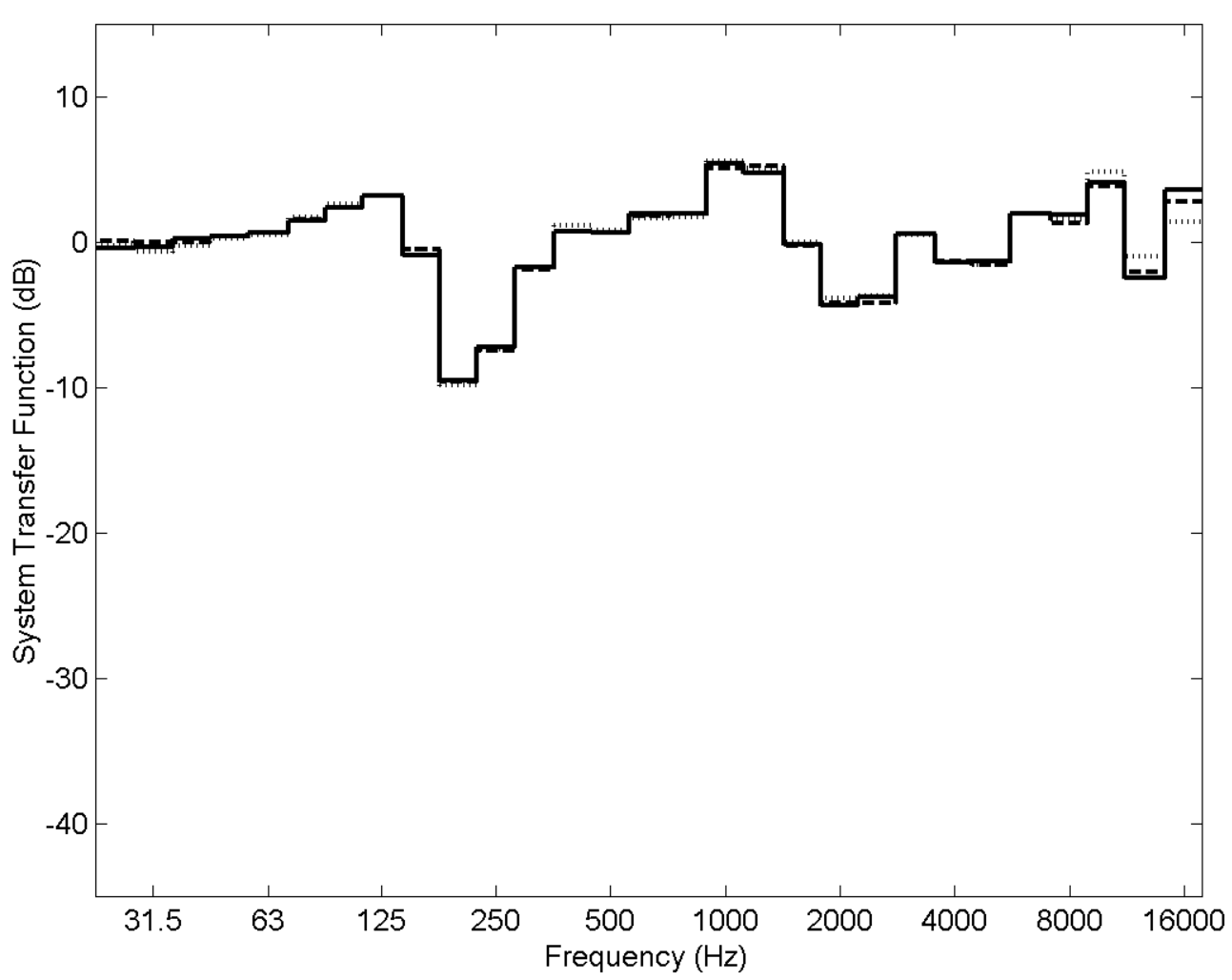

Figure 5.11: Transfer function for the TechnoFirst external ANC system, right ear in terms of one-third octave bands for different signals. - random noise signal; .. speech signal; -- traffic signal.

\subsubsection{Noise Reduction Performance}

Attenuation performance for hearing protectors is usually given in terms of a single number called the noise reduction rating (NRR). The calculation procedures are specified in Title 40 of the Code of Federal Regulation (CFR) part 211.207 (National Archives and Records Administration, 2001b). The testing procedures are adopted from ANSI S3.191974 which describes a subjective method to assess the performance of hearing protectors based upon hearing threshold levels (American National Standards Institute, 1974).

Adaptations were made to the NRR calculation procedures to utilize objective rather than subjective transfer function data. In addition, the summation of energy, when needed, 
was handled in an exact way instead of the approximate method described in the CFR. Table 5.1 lists the NRR as calculated via the modified method. The results indicate that only the NCT ProActive 3500 offers significant noise protection.

\begin{tabular}{|l|l|}
\hline ANC System & Modified NRR (dB) \\
\hline NCT Noise Buster Extreme, Max setting & 5 \\
\hline NCT ProActive 1500 & 1 \\
\hline NCT ProActive 3500 & 24 \\
\hline TechnoFirst external prototype & 0 \\
\hline
\end{tabular}

Table 5.1: NRR for the ANC systems.

\subsubsection{Speech Intelligibility Performance of ANC Systems}

The Speech Intelligibility Index for a given communication system is a measure of the percentage of the speech cues available to the listener in the presence of noise. The SII ranges from a maximum of 1.00 to a minimum of 0.00 . According to the standard, poor communication systems have a SII below 0.45 while good communication systems have a SII above 0.75 .

To investigate the overloading effect of high speech levels, the SII for the four vocal effort levels was calculated with no system gain and no external noise. The results are displayed in Table 5.2. The distance between the talker's lips and the listener's ears varied from 0.5 meters to 1 meter. As expected, the results show a perfect SII for the normal vocal effort. Due to overloading effects, the higher vocal efforts resulted in a slight decrease in the SII. 


\begin{tabular}{|l|l|l|l|l|l|}
\hline $\begin{array}{l}\text { System } \\
\text { Insertion } \\
\text { Gain }\end{array}$ & Distance (m) & Vocal Effort & $\begin{array}{l}\text { Equivalent } \\
\text { Noise }\end{array}$ & $\begin{array}{l}\text { Equivalent } \\
\text { Hearing } \\
\text { Threshold }\end{array}$ & SII \\
\hline None & 1.00 & Normal & None & Reference & 1.00 \\
\hline None & 1.00 & Raised & None & Reference & 1.00 \\
\hline None & 1.00 & Loud & None & Reference & 0.97 \\
\hline None & 1.00 & Shout & None & Reference & 0.92 \\
\hline None & 0.75 & Normal & None & Reference & 1.00 \\
\hline None & 0.75 & Raised & None & Reference & 1.00 \\
\hline None & 0.75 & Loud & None & Reference & 0.95 \\
\hline None & 0.75 & Shout & None & Reference & 0.90 \\
\hline None & 0.50 & Normal & None & Reference & 1.00 \\
\hline None & 0.50 & Raised & None & Reference & 0.98 \\
\hline None & 0.50 & Loud & None & Reference & 0.93 \\
\hline None & 0.50 & Shout & None & Reference & 0.88 \\
\hline
\end{tabular}

Table 5.2: SII results for no system gain, no external noise.

\subsubsection{Speech Intelligibility Performance for Typical Semi-Truck Noise}

The models were reevaluated with typical semi-truck traffic noise that included decelerating, idling, and accelerating vehicle conditions. The results are displayed in Table 5.3. All of the SII scores are below 0.45. It may be inferred that the toll plaza is a poor communication environment, even for the highest vocal effort. In the presence of typical semi-truck traffic noise, the vocal effort and the distance between the talker's lips and listener's ears become highly important to speech intelligibility. As shown in Figures 5.12 through 5.13, the vocal effort and the distance affect the signal to noise ratio. In band masking may be more important than low frequency masking. 


\begin{tabular}{|l|l|l|l|l|l|}
\hline $\begin{array}{l}\text { System } \\
\text { Insertion } \\
\text { Gain }\end{array}$ & Distance (m) & Vocal Effort & $\begin{array}{l}\text { Equivalent } \\
\text { Noise }\end{array}$ & $\begin{array}{l}\text { Equivalent } \\
\text { Hearing } \\
\text { Threshold }\end{array}$ & SII \\
\hline None & 1.00 & Normal & Traffic Noise & Reference & 0.00 \\
\hline None & 1.00 & Raised & Traffic Noise & Reference & 0.00 \\
\hline None & 1.00 & Loud & Traffic Noise & Reference & 0.06 \\
\hline None & 1.00 & Shout & Traffic Noise & Reference & 0.27 \\
\hline None & 0.75 & Normal & Traffic Noise & Reference & 0.00 \\
\hline None & 0.75 & Raised & Traffic Noise & Reference & 0.01 \\
\hline None & 0.75 & Loud & Traffic Noise & Reference & 0.12 \\
\hline None & 0.75 & Shout & Traffic Noise & Reference & 0.34 \\
\hline None & 0.50 & Normal & Traffic Noise & Reference & 0.00 \\
\hline None & 0.50 & Raised & Traffic Noise & Reference & 0.04 \\
\hline None & 0.50 & Loud & Traffic Noise & Reference & 0.21 \\
\hline None & 0.50 & Shout & Traffic Noise & Reference & 0.43 \\
\hline
\end{tabular}

Table 5.3: SII results for no system gain, actual traffic noise. 


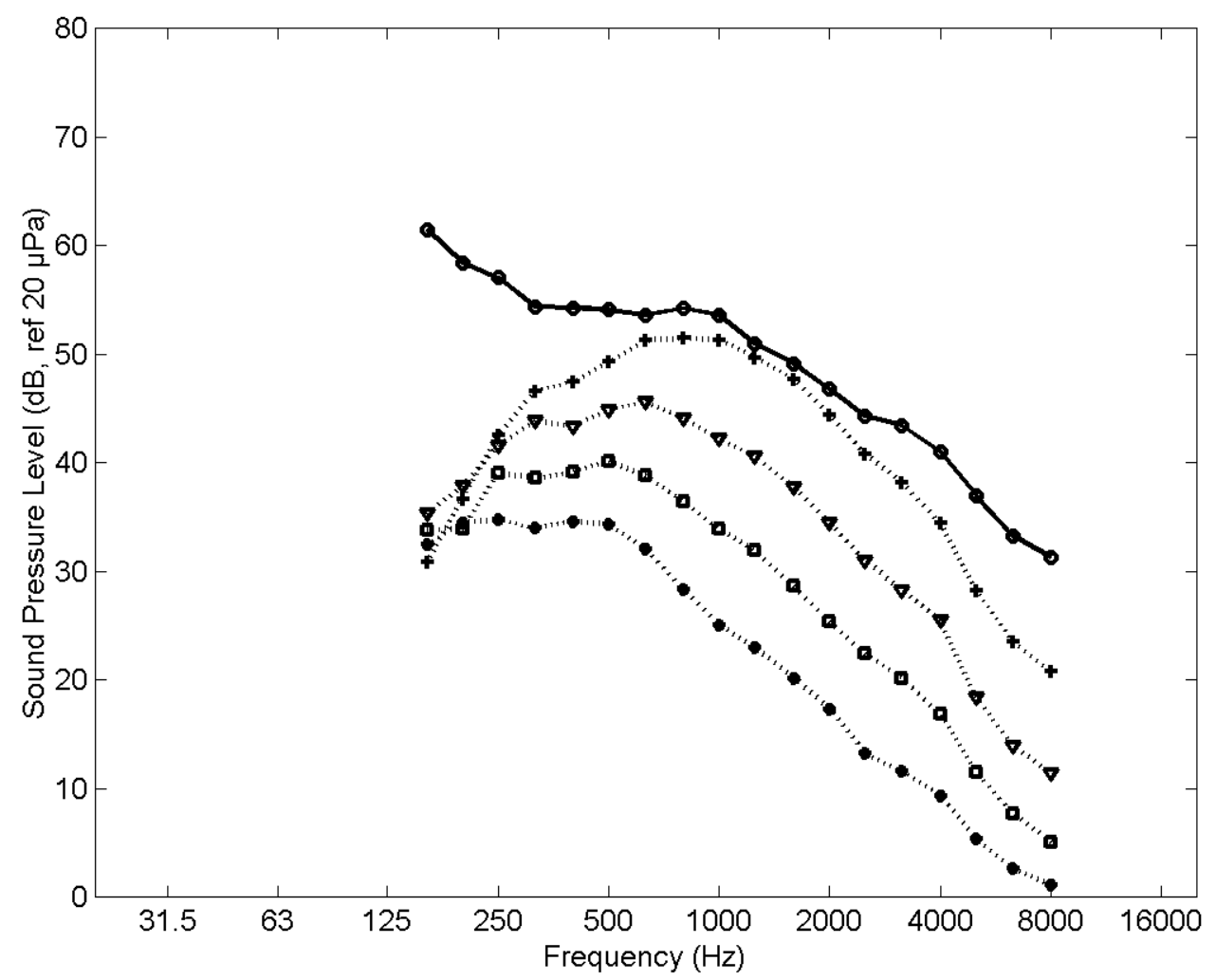

Figure 5.12: Comparison of semi-truck traffic spectrum level density with speech spectrum level density for a distance of 1.0 meters from talker's lips to listener's ears. X normal speech; raised speech; $\nabla$ loud speech; + shout speech; O semi-truck traffic noise. 


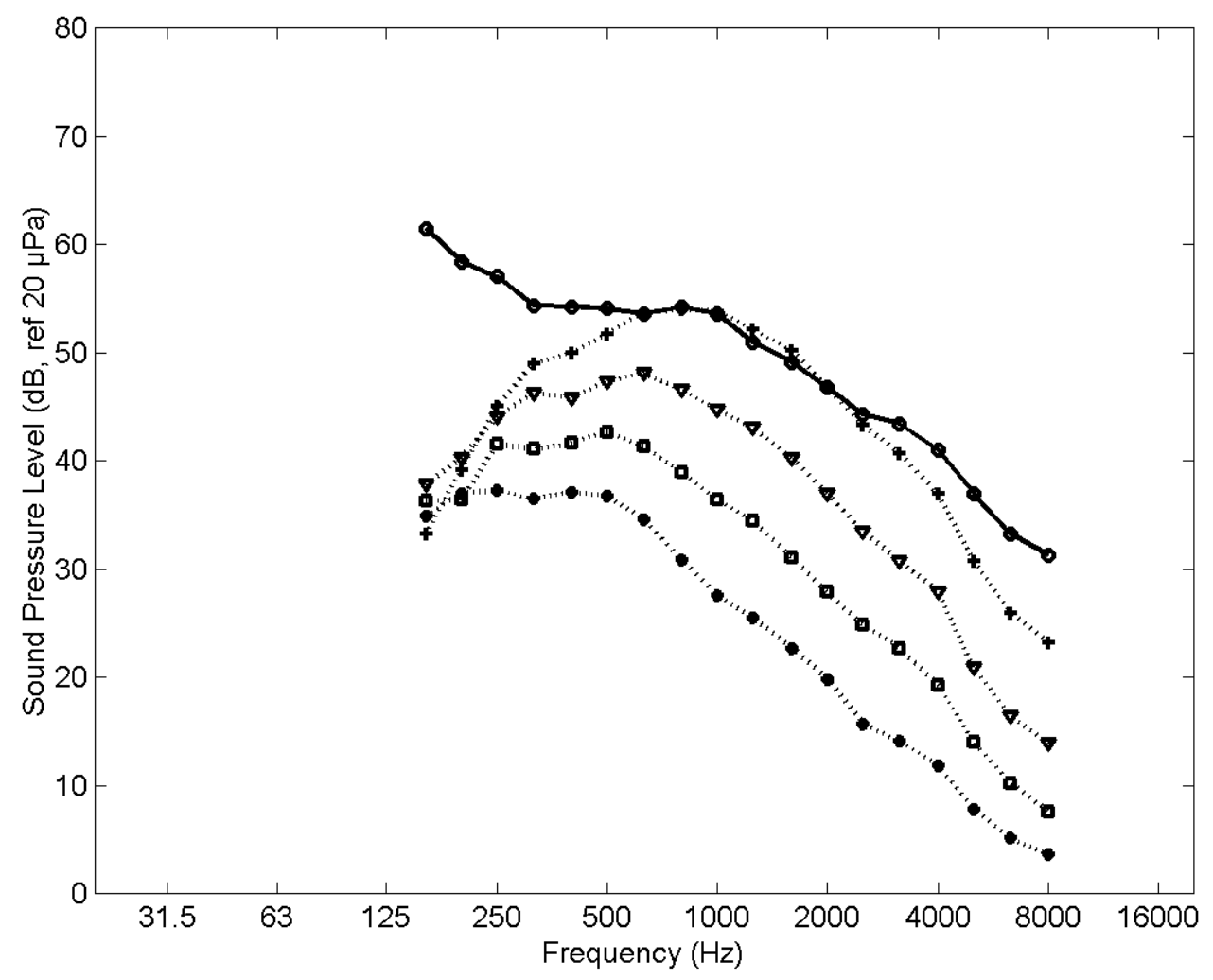

Figure 5.13: Comparison of semi-truck traffic spectrum level density with speech spectrum level density for a distance of 0.75 meters from talker's lips to listener's ears. X normal speech; raised speech; $\nabla$ loud speech; + shout speech; O semi-truck traffic noise. 


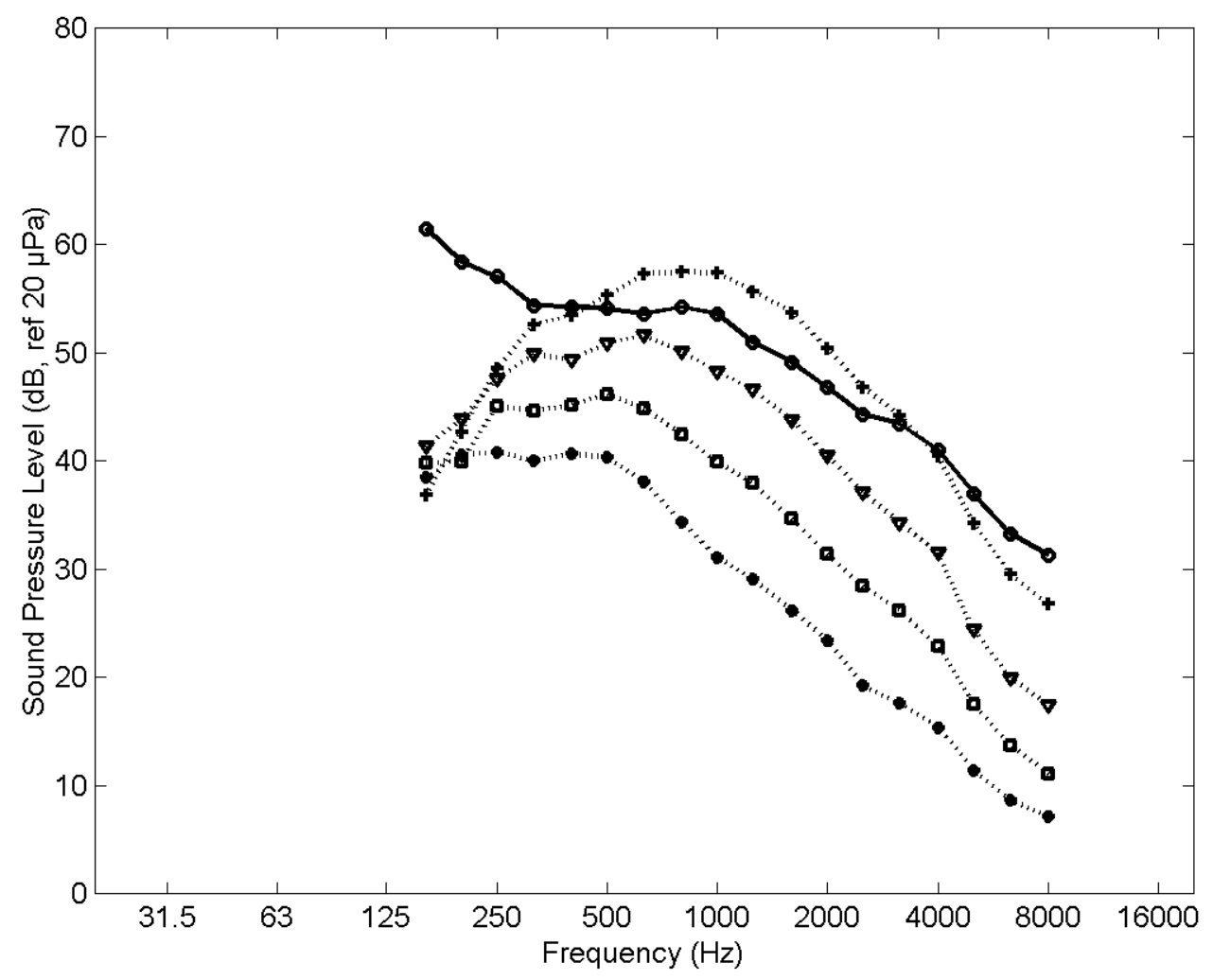

Figure 5.14: Comparison of semi-truck traffic spectrum level density with speech spectrum level density for a distance of 0.5 meters from talker's lips to listener's ears. X normal speech; raised speech; $\nabla$ loud speech; + shout speech; O semi-truck traffic noise.

Though the communication environment is acoustically poor, communication may be aided by other means such as gestures resulting in visual cues. The communication between tollbooth operators and patrons of the toll road is often simple and of a known context, which may improve information transfer. From observation, toll takers generally communicate with loud or shouting vocal effort. Despite these efforts to communicate, the number of incidences of miscommunication are high although communication is generally accomplished.

The effects of the ANC systems on the SII for tollbooth conditions was investigated. The results are displayed in Table 5.4. The results vary little from the 
previous case and suggest that all of the devices improve the SII a negligible amount. The system transfer functions equally affects both the noise spectrum and the vocal spectrum. Since both spectrums are affected equally, the overall dominance of the noise remains. Therefore the ANC systems do not directly improve the signal to noise ratio. The ANC systems can only be used to reduce low frequency masking effects and level overloading effects. The ProActive 3500 had the greatest benefit on the SII. Since the ProActive 3500 has high attenuation relative to the other active control systems, the results suggests that overloading of the ear may be an important factor for speech intelligibility in toll plaza applications. In some of the cases, the SII results went down with the use of NCT Noise Buster Extremes, NCT ProActive 1500, and the TechnoFirst prototype. All of these devices exhibit some amplification at particular bands. The slight decrease in intelligibility may be due to overloading effects with select frequency band amplification. 


\begin{tabular}{|c|c|c|c|c|c|}
\hline $\begin{array}{l}\text { System } \\
\text { Insertion Gain }\end{array}$ & Distance (m) & Vocal Effort & $\begin{array}{l}\text { Equivalent } \\
\text { Noise }\end{array}$ & $\begin{array}{l}\text { Equivalent } \\
\text { Hearing } \\
\text { Threshold }\end{array}$ & SII \\
\hline NB, Max & 1.00 & Normal & Traffic Noise & Reference & 0.00 \\
\hline PA 1500 & 1.00 & Normal & Traffic Noise & Reference & 0.00 \\
\hline PA 3500 & 1.00 & Normal & Traffic Noise & Reference & 0.00 \\
\hline TechnoFirst & 1.00 & Normal & Traffic Noise & Reference & 0.00 \\
\hline $\mathrm{NB}, \mathrm{Max}$ & 1.00 & Raised & Traffic Noise & Reference & 0.00 \\
\hline PA 1500 & 1.00 & Raised & Traffic Noise & Reference & 0.00 \\
\hline PA 3500 & 1.00 & Raised & Traffic Noise & Reference & 0.00 \\
\hline TechnoFirst & 1.00 & Raised & Traffic Noise & Reference & 0.00 \\
\hline NB, Max & 1.00 & Loud & Traffic Noise & Reference & 0.06 \\
\hline PA 1500 & 1.00 & Loud & Traffic Noise & Reference & 0.07 \\
\hline PA 3500 & 1.00 & Loud & Traffic Noise & Reference & 0.06 \\
\hline TechnoFirst & 1.00 & Loud & Traffic Noise & Reference & 0.05 \\
\hline NB, Max & 1.00 & Shout & Traffic Noise & Reference & 0.25 \\
\hline PA 1500 & 1.00 & Shout & Traffic Noise & Reference & 0.25 \\
\hline PA 3500 & 1.00 & Shout & Traffic Noise & Reference & 0.31 \\
\hline TechnoFirst & 1.00 & Shout & Traffic Noise & Reference & 0.26 \\
\hline NB, Max & 0.75 & Normal & Traffic Noise & Reference & 0.00 \\
\hline PA 1500 & 0.75 & Normal & Traffic Noise & Reference & 0.00 \\
\hline PA 3500 & 0.75 & Normal & Traffic Noise & Reference & 0.00 \\
\hline TechnoFirst & 0.75 & Normal & Traffic Noise & Reference & 0.00 \\
\hline NB, Max & 0.75 & Raised & Traffic Noise & Reference & 0.01 \\
\hline PA 1500 & 0.75 & Raised & Traffic Noise & Reference & 0.01 \\
\hline PA 3500 & 0.75 & Raised & Traffic Noise & Reference & 0.01 \\
\hline TechnoFirst & 0.75 & Raised & Traffic Noise & Reference & 0.01 \\
\hline NB, Max & 0.75 & Loud & Traffic Noise & Reference & 0.10 \\
\hline PA 1500 & 0.75 & Loud & Traffic Noise & Reference & 0.11 \\
\hline PA 3500 & 0.75 & Loud & Traffic Noise & Reference & 0.12 \\
\hline TechnoFirst & 0.75 & Loud & Traffic Noise & Reference & 0.10 \\
\hline NB, Max & 0.75 & Shout & Traffic Noise & Reference & 0.32 \\
\hline PA 1500 & 0.75 & Shout & Traffic Noise & Reference & 0.32 \\
\hline PA 3500 & 0.75 & Shout & Traffic Noise & Reference & 0.39 \\
\hline TechnoFirst & 0.75 & Shout & Traffic Noise & Reference & 0.33 \\
\hline NB, Max & 0.50 & Normal & Traffic Noise & Reference & 0.00 \\
\hline PA 1500 & 0.50 & Normal & Traffic Noise & Reference & 0.00 \\
\hline PA 3500 & 0.50 & Normal & Traffic Noise & Reference & 0.00 \\
\hline TechnoFirst & 0.50 & Normal & Traffic Noise & Reference & 0.00 \\
\hline NB, Max & 0.50 & Raised & Traffic Noise & Reference & 0.05 \\
\hline PA 1500 & 0.50 & Raised & Traffic Noise & Reference & 0.05 \\
\hline PA 3500 & 0.50 & Raised & Traffic Noise & Reference & 0.04 \\
\hline TechnoFirst & 0.50 & Raised & Traffic Noise & Reference & 0.04 \\
\hline NB, Max & 0.50 & Loud & Traffic Noise & Reference & 0.18 \\
\hline PA 1500 & 0.50 & Loud & Traffic Noise & Reference & 0.19 \\
\hline PA 3500 & 0.50 & Loud & Traffic Noise & Reference & 0.23 \\
\hline TechnoFirst & 0.50 & Loud & Traffic Noise & Reference & 0.20 \\
\hline NB, Max & 0.50 & Shout & Traffic Noise & Reference & 0.42 \\
\hline PA 1500 & 0.50 & Shout & Traffic Noise & Reference & 0.42 \\
\hline PA 3500 & 0.50 & Shout & Traffic Noise & Reference & 0.50 \\
\hline TechnoFirst & 0.50 & Shout & Traffic Noise & Reference & 0.42 \\
\hline
\end{tabular}

Table 5.4: SII results in the presence of actual semi-truck noise. 


\subsubsection{Speech Intelligibility Performance for Idle Truck Noise}

Communication primarily occurs while a vehicle is stationary and idling. As a realistic communication situation, idling semi-truck noise was used in the assessment of the ANC systems. The results are displayed in Table 5.5. There is a slight improvement, up to 0.03 , in the SII results. The small improvement suggests that the average spectrum is approximately the same as the idling noise spectrum. High acceleration levels are mostly canceled by low levels as traffic approaches the tollbooth. As a further investigation, the idling semi-truck noise spectrum was compared to the various vocal effort spectra in Figures 5.15 through 5.17 . 


\begin{tabular}{|c|c|c|c|c|c|}
\hline System Insertion Gain & Distance (m) & Vocal Effort & Equivalent Noise & $\begin{array}{l}\text { Equivalent Hearing } \\
\text { Threshold }\end{array}$ & SII \\
\hline None & 1.00 & Normal & Idle Truck & Reference & 0.00 \\
\hline NB, Max & 1.00 & Normal & Idle Truck & Reference & 0.00 \\
\hline PA 1500 & 1.00 & Normal & Idle Truck & Reference & 0.00 \\
\hline PA 3500 & 1.00 & Normal & Idle Truck & Reference & 0.00 \\
\hline TechnoFirst & 1.00 & Normal & Idle Truck & Reference & 0.00 \\
\hline None & 1.00 & Raised & Idle Truck & Reference & 0.00 \\
\hline NB, Max & 1.00 & Raised & Idle Truck & Reference & 0.00 \\
\hline PA 1500 & 1.00 & Raised & Idle Truck & Reference & 0.00 \\
\hline PA 3500 & 1.00 & Raised & Idle Truck & Reference & 0.00 \\
\hline TechnoFirst & 1.00 & Raised & Idle Truck & Reference & 0.00 \\
\hline None & 1.00 & Loud & Idle Truck & Reference & 0.07 \\
\hline $\mathrm{NB}$, Max & 1.00 & Loud & Idle Truck & Reference & 0.07 \\
\hline PA 1500 & 1.00 & Loud & Idle Truck & Reference & 0.08 \\
\hline PA 3500 & 1.00 & Loud & Idle Truck & Reference & 0.08 \\
\hline TechnoFirst & 1.00 & Loud & Idle Truck & Reference & 0.06 \\
\hline None & 1.00 & Shout & Idle Truck & Reference & 0.30 \\
\hline$\overline{N B}$, Max & 1.00 & Shout & Idle Truck & Reference & 0.27 \\
\hline$\overline{P A} 1500$ & 1.00 & Shout & Idle Truck & Reference & 0.28 \\
\hline PA 3500 & 1.00 & Shout & Idle Truck & Reference & 0.34 \\
\hline TechnoFirst & 1.00 & Shout & Idle Truck & Reference & 0.28 \\
\hline None & 0.75 & Normal & Idle Truck & Reference & 0.00 \\
\hline$\overline{N B}$, Max & 0.75 & Normal & Idle Truck & Reference & 0.00 \\
\hline PA 1500 & 0.75 & Normal & Idle Truck & Reference & 0.00 \\
\hline PA 3500 & 0.75 & Normal & Idle Truck & Reference & 0.00 \\
\hline TechnoFirst & 0.75 & Normal & Idle Truck & Reference & 0.00 \\
\hline None & 0.75 & Raised & Idle Truck & Reference & 0.01 \\
\hline NB, Max & 0.75 & Raised & Idle Truck & Reference & 0.02 \\
\hline PA 1500 & 0.75 & Raised & Idle Truck & Reference & 0.02 \\
\hline PA 3500 & 0.75 & Raised & Idle Truck & Reference & 0.02 \\
\hline TechnoFirst & 0.75 & Raised & Idle Truck & Reference & 0.01 \\
\hline None & 0.75 & Loud & Idle Truck & Reference & 0.14 \\
\hline NB, Max & 0.75 & Loud & Idle Truck & Reference & 0.12 \\
\hline PA 1500 & 0.75 & Loud & Idle Truck & Reference & 0.12 \\
\hline PA 3500 & 0.75 & Loud & Idle Truck & Reference & 0.15 \\
\hline TechnoFirst & 0.75 & Loud & Idle Truck & Reference & 0.12 \\
\hline None & 0.75 & Shout & Idle Truck & Reference & 0.37 \\
\hline NB, Max & 0.75 & Shout & Idle Truck & Reference & 0.35 \\
\hline$\overline{P A} 1500$ & 0.75 & Shout & Idle Truck & Reference & 0.35 \\
\hline PA 3500 & 0.75 & Shout & Idle Truck & Reference & 0.42 \\
\hline TechnoFirst & 0.75 & Shout & Idle Truck & Reference & 0.36 \\
\hline None & 0.50 & Normal & Idle Truck & Reference & 0.00 \\
\hline NB, Max & 0.50 & Normal & Idle Truck & Reference & 0.00 \\
\hline PA 1500 & 0.50 & Normal & Idle Truck & Reference & 0.00 \\
\hline PA 3500 & 0.50 & Normal & Idle Truck & Reference & 0.00 \\
\hline TechnoFirst & 0.50 & Normal & Idle Truck & Reference & 0.00 \\
\hline None & 0.50 & Raised & Idle Truck & Reference & 0.05 \\
\hline$\overline{\mathrm{NB}}, \mathrm{Max}$ & 0.50 & Raised & Idle Truck & Reference & 0.06 \\
\hline PA 1500 & 0.50 & Raised & Idle Truck & Reference & 0.06 \\
\hline PA 3500 & 0.50 & Raised & Idle Truck & Reference & 0.05 \\
\hline TechnoFirst & 0.50 & Raised & Idle Truck & Reference & 0.05 \\
\hline None & 0.50 & Loud & Idle Truck & Reference & 0.24 \\
\hline$\overline{N B}$, Max & 0.50 & Loud & Idle Truck & Reference & 0.20 \\
\hline PA 1500 & 0.50 & Loud & Idle Truck & Reference & 0.21 \\
\hline PA 3500 & 0.50 & Loud & Idle Truck & Reference & 0.26 \\
\hline TechnoFirst & 0.50 & Loud & Idle Truck & Reference & 0.22 \\
\hline None & 0.50 & Shout & Idle Truck & Reference & 0.46 \\
\hline NB, Max & 0.50 & Shout & Idle Truck & Reference & 0.45 \\
\hline PA 1500 & 0.50 & Shout & Idle Truck & Reference & 0.44 \\
\hline PA 3500 & 0.50 & Shout & Idle Truck & Reference & 0.53 \\
\hline TechnoFirst & 0.50 & Shout & Idle Truck & Reference & 0.45 \\
\hline
\end{tabular}

Table 5.5: SII results in the presence of idle semi-truck noise. 


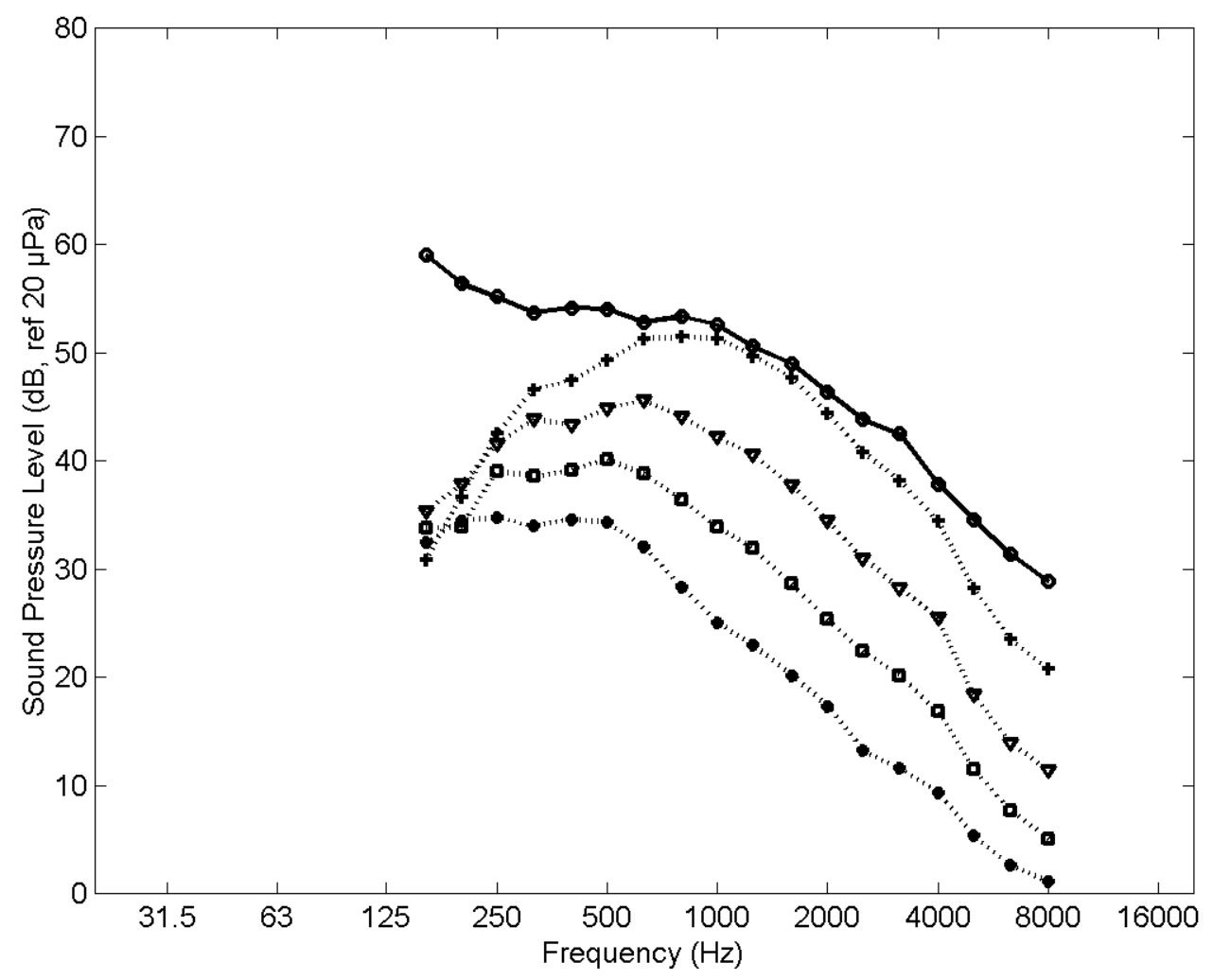

Figure 5.15: Comparison of idling semi-truck traffic spectrum level density with speech spectrum level density for a distance of 1.0 meters from talker's lips to listener's ears. X normal speech; raised speech; $\nabla$ loud speech; + shout speech; O idling semi-truck traffic noise. 


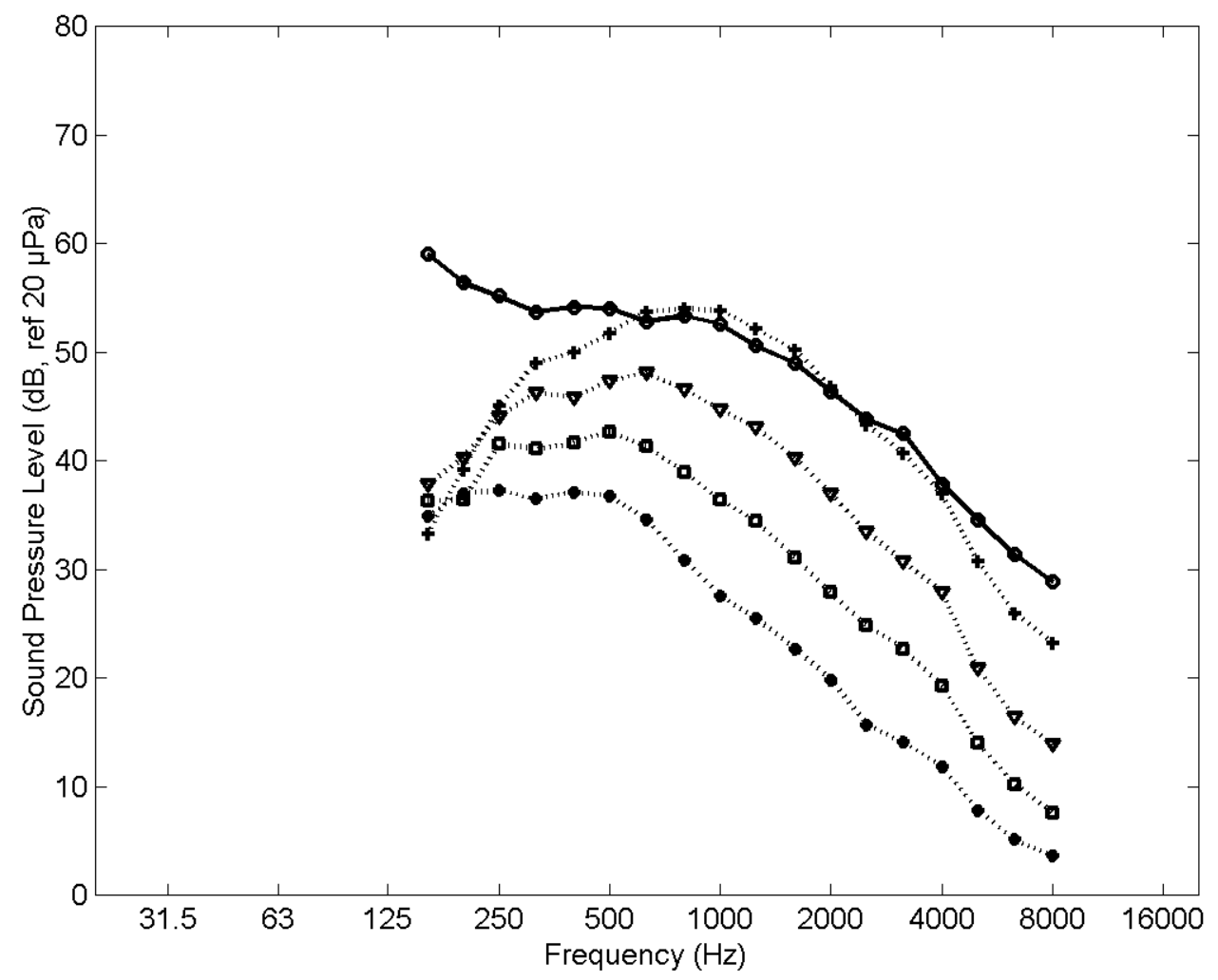

Figure 5.16: Comparison of idling semi-truck traffic spectrum level density with speech spectrum level density for a distance of 0.75 meters from talker's lips to listener's ears. X normal speech; raised speech; $\nabla$ loud speech; + shout speech; O idling semi-truck traffic noise. 


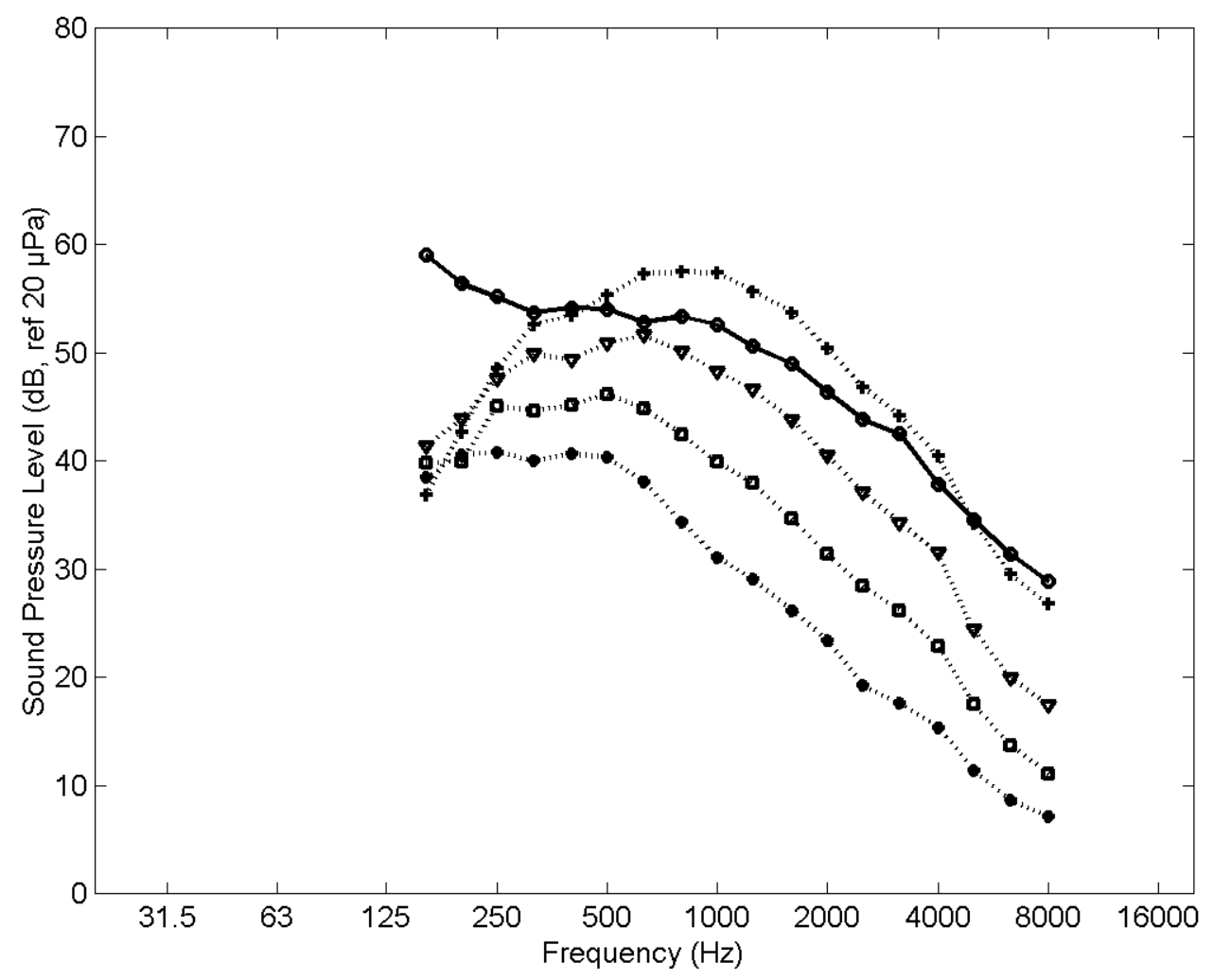

Figure 5.17: Comparison of idling semi-truck traffic spectrum level density with speech spectrum level density for a distance of 0.5 meters from talker's lips to listener's ears. X normal speech; raised speech; $\nabla$ loud speech; + shout speech; O idling semi-truck traffic noise.

\subsubsection{Speech Intelligibility Performance for Idle Car Noise}

TNM data was used to develop a transfer function between idling semi-truck noise and idling passenger vehicle noise. The SII in the presence of car noise without ANC systems was investigated. A listing of the results is shown in Table 5.6. Car noise does not pose a problem for speech intelligibility. This prediction is consistent with the survey responses of the toll plaza employees discussed in Chapter 2. The various vocal effort spectra are compared to idling car noise spectrum in Figures 5.18 through 5.20. 


\begin{tabular}{|l|l|l|l|l|l|}
\hline $\begin{array}{l}\text { System } \\
\text { Insertion } \\
\text { Gain }\end{array}$ & Distance (m) & Vocal Effort & $\begin{array}{l}\text { Equivalent } \\
\text { Noise }\end{array}$ & $\begin{array}{l}\text { Equivalent } \\
\text { Hearing } \\
\text { Threshold }\end{array}$ & SII \\
\hline None & 1.00 & Normal & Idle Car & Reference & 0.36 \\
\hline None & 1.00 & Raised & Idle Car & Reference & 0.60 \\
\hline None & 1.00 & Loud & Idle Car & Reference & 0.83 \\
\hline None & 1.00 & Shout & Idle Car & Reference & 0.91 \\
\hline None & 0.75 & Normal & Idle Car & Reference & 0.44 \\
\hline None & 0.75 & Raised & Idle Car & Reference & 0.68 \\
\hline None & 0.75 & Loud & Idle Car & Reference & 0.89 \\
\hline None & 0.75 & Shout & Idle Car & Reference & 0.89 \\
\hline None & 0.50 & Normal & Idle Car & Reference & 0.56 \\
\hline None & 0.50 & Raised & Idle Car & Reference & 0.79 \\
\hline None & 0.50 & Loud & Idle Car & Reference & 0.93 \\
\hline None & 0.50 & Shout & Idle Car & Reference & 0.88 \\
\hline
\end{tabular}

Table 5.6: SII results in the presence of idle car noise. 


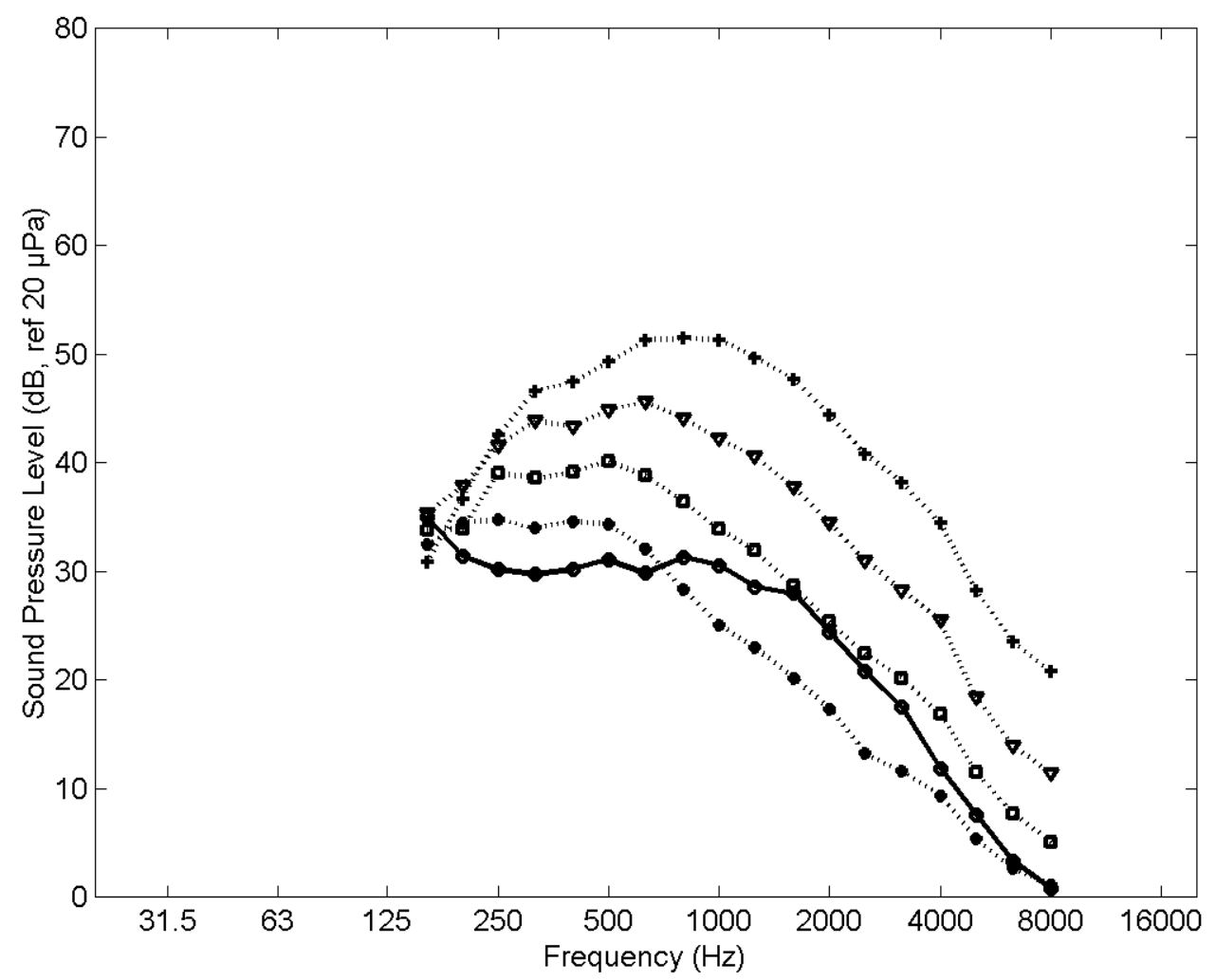

Figure 5.18: Comparison of idling car traffic spectral density with speech spectral density for a distance of 1.0 meters from talker's lips to listener's ears. X normal speech; raised speech; $\nabla$ loud speech; + shout speech; O idling car traffic noise. 


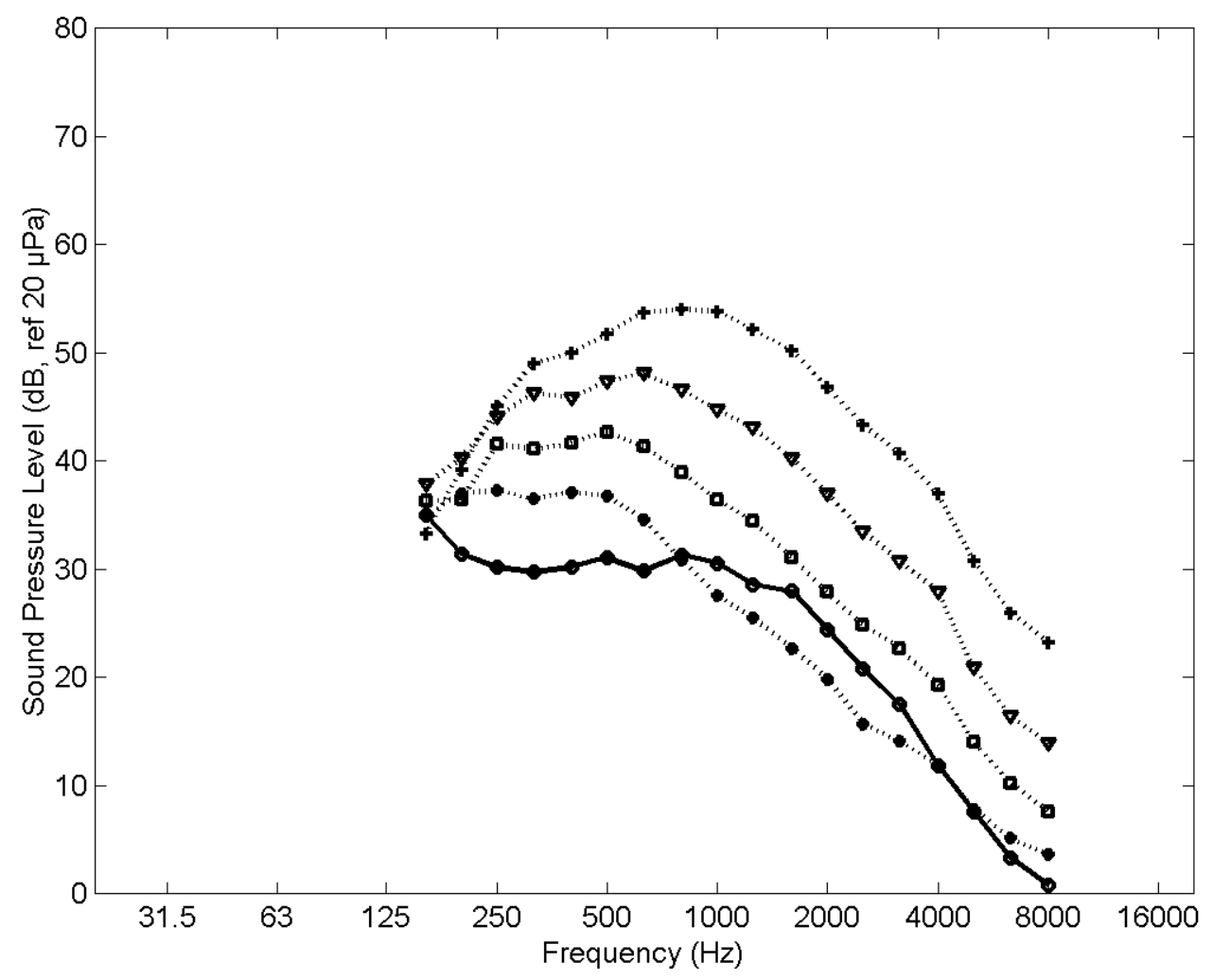

Figure 5.19: Comparison of idling car traffic spectrum level density with speech spectrum level density for a distance of 0.75 meters from talker's lips to listener's ears. $\mathrm{X}$ normal speech; $\quad$ raised speech; $\nabla$ loud speech; + shout speech; O idling car traffic noise. 


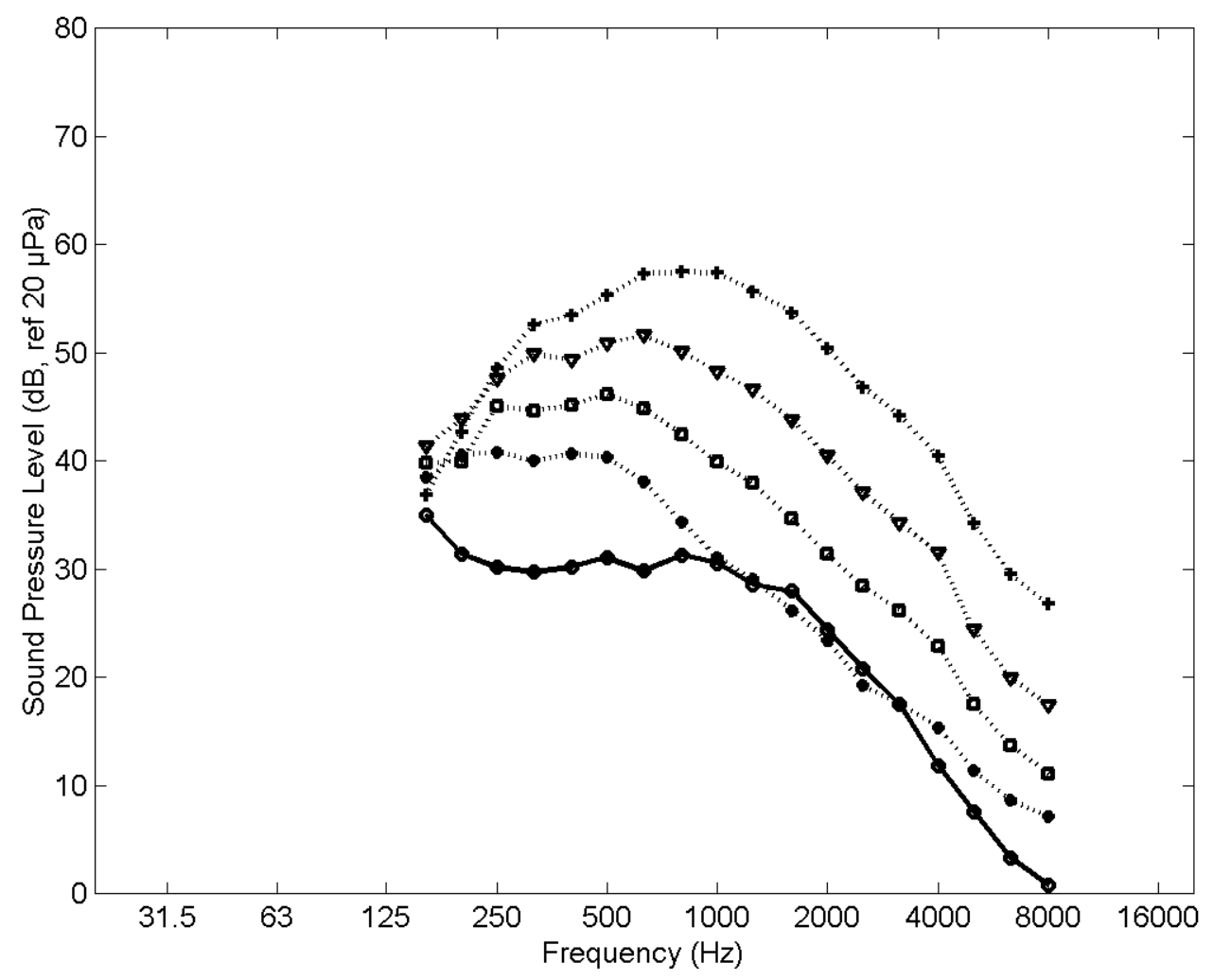

Figure 5.20: Comparison of idling car traffic spectrum level density with speech spectrum level density for a distance of 0.5 meters from talker's lips to listener's ears. $\mathrm{X}$ normal speech; $\quad$ raised speech; $\nabla$ loud speech; +shout speech; O idling car traffic noise.

\subsubsection{Characteristics of Systems for Improved Communication}

The predicted speech intelligibility results for toll plaza conditions suggest that semi-truck noise tends to dominate the sound field and overload the ear. To address the poor signal to noise ratio and the overloading effect, improved systems were investigated.

Realistic models and results are discussed in the following sections.

\subsubsection{Concepts for Improved Communication Systems}


Modeling of a directional microphone attached to a sealed ANC headset was explored to improve the signal to noise ratio. The improved system was created to attenuate environmental noise with the headset and selectively reinforce speech with a directional microphone. Closed ear, sealed headsets offer strong passive noise reduction at high frequencies. To attenuate all frequencies, a sealed ANC headset like the NCT ProActive 3500 is desirable. While the ANC headset reduces the level it does not directly increase the signal to noise ratio. Directional microphones exhibit a greater sensitivity for sounds in a particular direction than for omni-directional sounds. A realistic implementation of a dipole directional microphone would yield a $6 \mathrm{~dB}$ increase for sounds incident from the front. Therefore frontal sounds, such as the vocalization from toll road patrons, would be increased by $6 \mathrm{~dB}$ over the more omni-directional traffic noise. By attaching the output signal of a directional microphone to the acoustic drivers within a seal ANC headset, the signal to noise ratio would be improved.

A $6 \mathrm{~dB}$ increase in signal over noise produces the same signal levels as reducing the distance between the talker's lips and the listener's ears by a factor of two. The positive speech intelligibility effects by decreasing the distance have been illustrated in previous sections. Modeling analog filters inline with the directional microphone offers further control of the signal reaching the attendants ears. By filtering the signal, masking and overloading effects can be further investigated.

\subsubsection{Sealed Headset with Directional Microphone}

The sealed ANC headset with a dipole directional microphone was modeled using a $0 \mathrm{~dB}$ insertion gain for the noise and amplifying the speech signal by $6 \mathrm{~dB}$. Therefore the noise level is the same as that without the headsets, but the speech was increased by $6 \mathrm{~dB}$. This concept assumes that the microphone input would dominate the sound entering the ear 
cup of the headset and make the insertion gain of the sealed ANC headset negligible. It also assumes that the gain of the microphone system would be set to bring the noise level inside the ear cup to the same level as the case with no noise control solution. The SII results for

the improved systems are displayed in Table 5.7. Comparing the results from Table 5.5 and Table 5.7, the use of a sealed ANC headset with a single dipole directional microphone can yield as much as a 0.20 increase in SII for loud and shout vocal efforts which are common at the toll plaza. This improvement is markedly better than that obtained for any of the tested ANC systems.

\subsubsection{Filtering the Directional Microphone Signal}

Low frequency masking effects were investigated by modeling a high pass filter for the directional microphone signal. The transfer function of the high pass filter attenuated the $160 \mathrm{~Hz}$ to $315 \mathrm{~Hz}$ bands by $30 \mathrm{~dB}$. These frequency bands were selected based upon high noise level and low speech importance. The results are displayed in Table 5.7. Using a high pass filter for the directional microphone signal seems to improve the SII, but often less than 0.01 .

A band pass filter was modeled using the low pass filter insertion gain with an additional attenuation of $30 \mathrm{~dB}$ in the $6300 \mathrm{~Hz}$ to $8000 \mathrm{~Hz}$ bands. These frequency bands were selected based upon high noise level and low band importance. The band pass filter appeared to reduce the SII. Low frequency sounds tend to mask higher frequency sound more than high frequency sounds tend to mask low frequency sounds. The masking profile due to a pure tone is display in Figure 5.21. Therefore attenuating high frequencies reduces the amount of information and in turn the SII while attenuating low frequencies may slightly improve the SII. For traffic noise, the spreading effects of masking are only important when a positive signal to noise ratio exists in band. As an example, when $500 \mathrm{~Hz}$ noise 
dominates $500 \mathrm{~Hz}$ speech, the masking of effect of a lower frequency sound are not as significant as the poor signal to noise ratio at $500 \mathrm{~Hz}$.

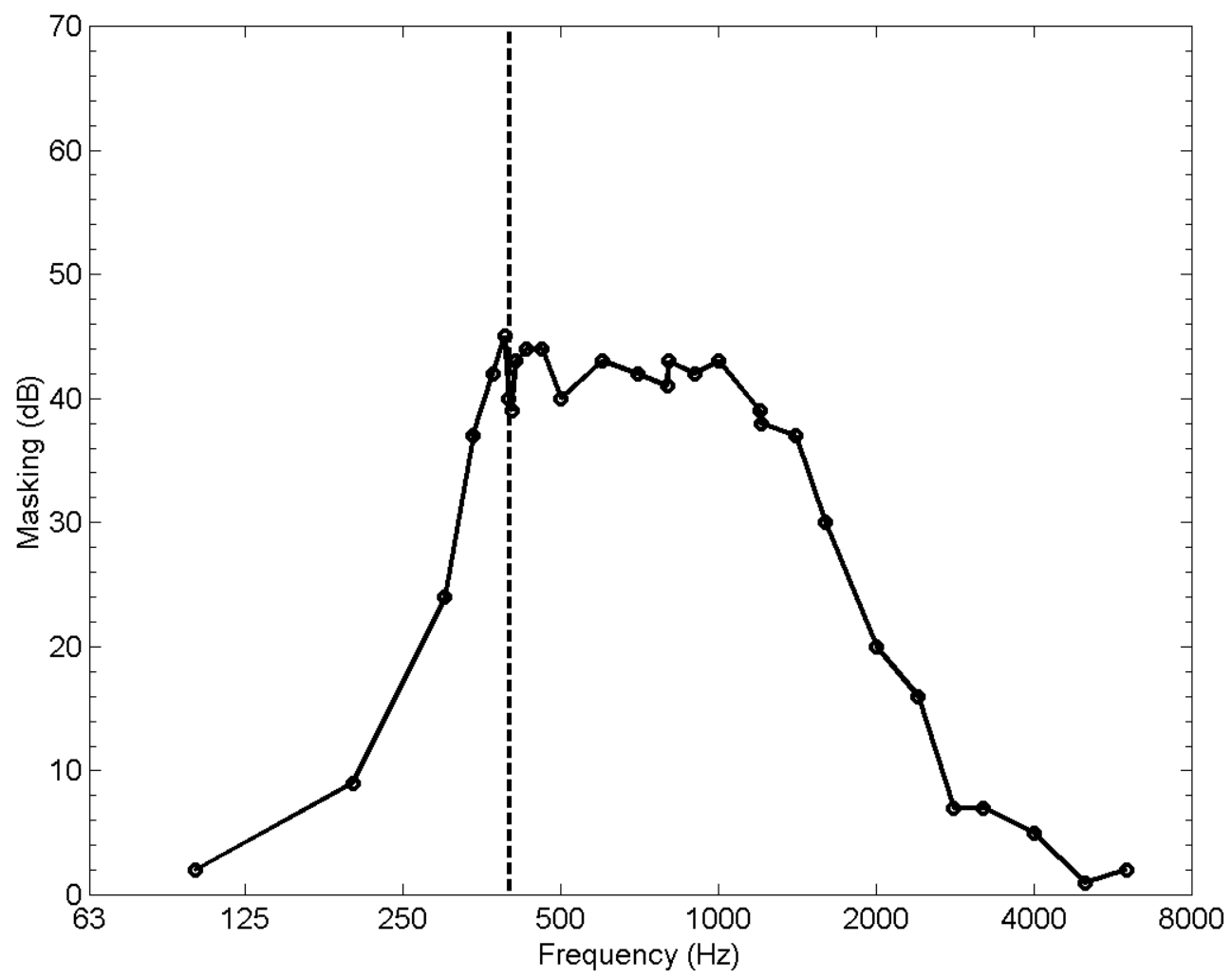

Figure 5.21: Masking profile due to a pure tone of $400 \mathrm{~Hz}$ at $80 \mathrm{~dB}$. Data from Egan and Hake (1950, p. 623).

Overloading effects and amplitude control for the directional microphone signal were modeled with a $-5 \mathrm{~dB}$ insertion gain and a flat $-10 \mathrm{~dB}$ insertion gain. Transfer functions with greater attenuation not were not believed to be desirable. The greater the attenuation of the directional microphone signal, the more significant the transfer function of the sealed ANC headset becomes. In addition, the selected attenuation levels were near the gain provided by the dipole directional microphone. A greater attenuation could cause the toll booth attendant to speak at a level too low for the toll road customer to understand. The 
results are displayed in Table 5.7. Reducing the level of the sound entering the ear improved the SII by as much as 0.05 . This is approximately the same improvement in SII observed for the NCT ProActive 3500 as demonstrated in Table5.5. The results indicate that overloading is more important than low frequency masking.

As a final study, the low pass filter was combined with the flat $-10 \mathrm{~dB}$ insertion gain. The insertion gain characteristics were $-30 \mathrm{~dB}$ in the $160 \mathrm{~Hz}$ to $315 \mathrm{~Hz}$ frequency bands, with $-10 \mathrm{~dB}$ in the remaining frequency bands. The results are displayed in Table 5.7. The combined filter seems to improve the SII, but less than 0.01 over the level control alone. The results confirm that overloading is more important that low frequency masking. 


\begin{tabular}{|c|c|c|c|c|c|}
\hline $\begin{array}{l}\text { System Insertion } \\
\text { Gain }\end{array}$ & $\begin{array}{l}\text { Distance } \\
\text { (m) }\end{array}$ & \begin{tabular}{|l|} 
Vocal \\
Effort
\end{tabular} & $\begin{array}{l}\text { Equivalent } \\
\text { Noise }\end{array}$ & $\begin{array}{l}\text { Equivalent Hearing } \\
\text { Threshold }\end{array}$ & SII \\
\hline $\begin{array}{l}\text { Sealed Headset with } \\
\text { Directional Mic }\end{array}$ & 0.75 & Normal & Idle Truck & Reference & 0.02 \\
\hline $\begin{array}{l}\text { System with high pass } \\
\text { filter }\end{array}$ & 0.75 & Normal & Idle Truck & Reference & 0.02 \\
\hline $\begin{array}{l}\text { System with band pass } \\
\text { filter }\end{array}$ & 0.75 & \begin{tabular}{|l|} 
Normal \\
\end{tabular} & Idle Truck & Reference & 0.02 \\
\hline $\begin{array}{l}\text { System with } 5 \mathrm{~dB} \\
\text { reduction }\end{array}$ & 0.75 & Normal & Idle Truck & Reference & 0.02 \\
\hline $\begin{array}{l}\text { System with } 10 \mathrm{~dB} \\
\text { reduction }\end{array}$ & 0.75 & Normal & Idle Truck & Reference & 0.02 \\
\hline $\begin{array}{l}\text { System with } 10 \mathrm{~dB} \\
\text { reduction and high pass }\end{array}$ & 0.75 & Normal & Idle Truck & Reference & 0.02 \\
\hline $\begin{array}{l}\text { Sealed Headset with } \\
\text { Directional Mic }\end{array}$ & 0.75 & Raised & Idle Truck & Reference & 0.09 \\
\hline $\begin{array}{l}\text { System with high pass } \\
\text { filter }\end{array}$ & 0.75 & Raised & Idle Truck & Reference & 0.09 \\
\hline $\begin{array}{l}\text { System with band pass } \\
\text { filter }\end{array}$ & 0.75 & Raised & Idle Truck & Reference & 0.09 \\
\hline $\begin{array}{l}\text { System with } 5 \mathrm{~dB} \\
\text { reduction }\end{array}$ & 0.75 & Raised & Idle Truck & Reference & 0.10 \\
\hline $\begin{array}{l}\text { System with } 10 \mathrm{~dB} \\
\text { reduction }\end{array}$ & 0.75 & Raised & Idle Truck & Reference & 0.10 \\
\hline $\begin{array}{l}\text { System with } 10 \mathrm{~dB} \\
\text { reduction and high pass }\end{array}$ & 0.75 & Raised & Idle Truck & Reference & 0.10 \\
\hline $\begin{array}{l}\text { Sealed Headset with } \\
\text { Directional Mic }\end{array}$ & 0.75 & Loud & Idle Truck & Reference & 0.31 \\
\hline $\begin{array}{l}\text { System with high pass } \\
\text { filter }\end{array}$ & 0.75 & Loud & Idle Truck & Reference & 0.31 \\
\hline $\begin{array}{l}\text { System with band pass } \\
\text { filter }\end{array}$ & 0.75 & \begin{tabular}{|l|} 
Loud \\
\end{tabular} & Idle Truck & Reference & 0.30 \\
\hline $\begin{array}{l}\text { System with } 5 \mathrm{~dB} \\
\text { reduction }\end{array}$ & 0.75 & \begin{tabular}{|l|} 
Loud \\
\end{tabular} & Idle Truck & Reference & 0.32 \\
\hline $\begin{array}{l}\text { System with } 10 \mathrm{~dB} \\
\text { reduction }\end{array}$ & 0.75 & \begin{tabular}{|l|} 
Loud \\
\end{tabular} & Idle Truck & Reference & 0.34 \\
\hline $\begin{array}{l}\text { System with } 10 \mathrm{~dB} \\
\text { reduction and high pass }\end{array}$ & 0.75 & Loud & Idle Truck & Reference & 0.34 \\
\hline $\begin{array}{l}\text { Sealed Headset with } \\
\text { Directional Mic }\end{array}$ & 0.75 & Shout & Idle Truck & Reference & 0.52 \\
\hline $\begin{array}{l}\text { System with high pass } \\
\text { filter }\end{array}$ & 0.75 & Shout & Idle Truck & Reference & 0.53 \\
\hline $\begin{array}{l}\text { System with band pass } \\
\text { filter }\end{array}$ & 0.75 & Shout & Idle Truck & Reference & 0.50 \\
\hline $\begin{array}{l}\text { System with } 5 \mathrm{~dB} \\
\text { reduction }\end{array}$ & 0.75 & Shout & Idle Truck & Reference & 0.55 \\
\hline $\begin{array}{l}\text { System with } 10 \mathrm{~dB} \\
\text { reduction }\end{array}$ & 0.75 & Shout & Idle Truck & Reference & 0.57 \\
\hline $\begin{array}{l}\text { System with } 10 \mathrm{~dB} \\
\text { reduction and high pass }\end{array}$ & 0.75 & Shout & Idle Truck & Reference & 0.57 \\
\hline
\end{tabular}

Table 5.7: SII results for improved systems in the presence of idle semi-truck noise. 


\subsubsection{Directional Microphone Directivity Index}

To make significant improvements to the speech intelligibility at the toll plaza, a system should create a positive signal to noise ratio and must attenuate the level reaching the ears to minimize overloading effects.

The microphone directivity index needed to produce a good communication system for normal vocal effort was explored. A pair of microphones spaced 0.03 meters and positioned in a dipole configuration creates the $6 \mathrm{~dB}$ gain for on axis sounds versus omnidirectional sounds discussed in the previous sections. Sets of dipole microphones can be used to create a higher order configuration with greater directivity. A distance of 0.75 meters between the talker's lips and the listener's ears was selected for this investigation. To address overloading effects, greater attenuation was used than in the previous study. The signal amplitude was set such that noise with the headset is $20 \mathrm{~dB}$ below the noise level without the headset. The experienced noise level with the tested system, therefore, is near that with a sealed ANC headset alone. The directional microphone configurations will augment the speech above this noise level. Again, it was assumed that the microphone input would dominate the sound entering the ear cup of the headset and make the insertion gain of the sealed ANC headset negligible. Though not as justified as in the previous study, such an assumption is satisfactory for this idealized study. Result of the investigation are displayed in Table 5.8. The results show that a high order directional microphone with a directivity index of $36 \mathrm{~dB}$ is sufficient to create a good communication system under normal vocal effort and realistic distances with $20 \mathrm{~dB}$ of microphone signal attenuation. 


\begin{tabular}{|l|l|l|l|l|l|l|}
\hline $\begin{array}{l}\text { Directivity } \\
\text { Index (dB) }\end{array}$ & $\begin{array}{l}\text { System } \\
\text { Insertion Gain }\end{array}$ & $\begin{array}{l}\text { Distance } \\
(\mathbf{m})\end{array}$ & $\begin{array}{l}\text { Vocal } \\
\text { Effort }\end{array}$ & $\begin{array}{l}\text { Equivalent } \\
\text { Noise }\end{array}$ & $\begin{array}{l}\text { Equivalent } \\
\text { Hearing } \\
\text { Threshold }\end{array}$ & SII \\
\hline 6 & $\begin{array}{l}\text { Sealed Headset } \\
\text { with Directional } \\
\text { Mic and 20 dB } \\
\text { reduction }\end{array}$ & 0.75 & Normal & Idle Truck & Reference & 0.02 \\
\hline 12 & $\begin{array}{l}\text { Sealed Headset } \\
\text { with Directional } \\
\text { Mic and 20 dB } \\
\text { reduction }\end{array}$ & 0.75 & Normal & Idle Truck & Reference & 0.08 \\
\hline 18 & $\begin{array}{l}\text { Sealed Headset } \\
\text { with Directional } \\
\text { Mic and 20 dB } \\
\text { reduction }\end{array}$ & 0.75 & Normal & Idle Truck & Reference & 0.26 \\
\hline $\begin{array}{l}\text { Sealed Headset } \\
\text { with Directional } \\
\text { Mic and 20 dB } \\
\text { reduction }\end{array}$ & $\begin{array}{l}\text { Sealed Headset } \\
\text { with Directional } \\
\text { Mic and 20 dB } \\
\text { reduction }\end{array}$ & 0.75 & Normal & Idle Truck & Reference & 0.65 \\
\hline 30 & $\begin{array}{l}\text { Sealed Headset } \\
\text { with Directional } \\
\text { Mic and 20 dB } \\
\text { reduction }\end{array}$ & 0.75 & Normal & Idle Truck & Reference & 0.80 \\
\hline 36 & & & & & & \\
\hline
\end{tabular}

Table 5.8: SII results for improved systems in the presence of idle semi-truck noise.

As a concluding thought, the changes in level produced by the improved ANC headset configurations may make it more difficult for customers to understand toll road employees. Upon wearing the headset, the noise level is lowered and the speech is easily understood. The tollbooth employee may be inclined to speak with a normal vocal effort. At this level, the employees' speech will be overpowered by the traffic noise and will not be intelligible to the toll road patrons. To address this potential problem, loudspeakers could be attached to the tollbooth which could be used to reinforce the speech of the tollbooth employee. 


\section{$\underline{5.5 \text { Summary }}$}

The ANSI SII calculation procedures for the one-third octave band method and direct measurement of the input data were coded. The insertion gain of three ANC headsets and an external ANC system was measured in the presence of three test signals. The NRR for the headsets was calculated in a modified manner to account for the objective transfer function data obtained for each system. The NCT Noise Buster Extreme produced a NRR of 5 while the NCT ProActive produced a NRR of 24. The remaining systems did not yield noteworthy NRR scores.

Without any of the ANC systems used, which is the current condition at the toll plaza, the SII results indicated that the environment was poor with respect to communication in the presence of semi-truck noise. With the system transfer functions of the ANC systems added, the SII changed very little. The results suggest that the semi-truck traffic noise level at the toll plaza overpowers the speech spectrum level and creates a low signal to noise ratio. The results for idle car noise show that passenger cars noise does not present a problem for speech intelligibility.

Improved systems which increased the signal to noise ratio were modeled and tested. The results indicate that use of a sealed ANC headset with a single dipole directional microphone yield a significant improvement in SII, as much a 0.20 increase, for vocal efforts which are common at the toll plaza. In the context of speech intelligibility in the presence of toll plaza noise, it was found that level overloading effects are more significant than low frequency masking and must be addressed in the solutions. Results from higher order microphone directivity investigation demonstrated that a good communication system (a SII of 0.75 or above) is achievable for normal vocal effort. 


\section{CONCLUSIONS AND RECOMMENDATIONS}

In this chapter, conclusions drawn from this research and recommendations for future work will be presented.

\section{$\underline{6.1 \text { Conclusions }}$}

Survey results confirmed that noise at toll plazas creates an unpleasant work environment and hinders communication. The results also suggest that the level may be near levels known to cause hearing damage. Passive noise control solutions were considered including: construction of partial barriers with a sound absorbing surface treatment, application of sound absorbing material to the overhead canopy, and treatment of exterior tollbooth walls with sound absorbing material. A beam tracing method was employed to model the noise conditions and test reasonable architectural noise control solution.

Verification studies suggested that beam tracing methods offer the potential to accurately model the ambient conditions around toll plazas and to evaluate the impact of possible noise control solutions. A detailed model of a specific, existing toll plaza located near Portage, Indiana was developed. The model considered the effects of the tollbooths, the canopy, ground reflections, diffraction around obstacles, and sound absorption from different materials. As a worst case scenario, semi-truck sources for idling and accelerating conditions were assumed. Source strengths were established using FHWA's TNM software. 
The plaza model results suggest that the maximum achievable noise reduction using passive methods is $3 \mathrm{~dB}$, a noticeable but rather modest decrease. In borderline circumstances, $3 \mathrm{~dB}$ may be enough to comply with noise exposure regulations or improve the environment. The results further suggest that the direct field accounts for all by 3 to 4 $\mathrm{dB}$ of the sound pressure level experienced by the tollbooth operators. To reduce the noise level significantly, noise control solution must be aimed at reducing the direct field. The modeling approach did not account for coherent reflections that cause reverberation and resonance. If resonance conditions occur, placement of absorbing material may have a more dramatic effect than predicted by the model.

The performance of single channel feedback ANC systems was investigated. Such devices address some of the weaknesses inherent in passive noise control solutions, however the impact of such systems on speech intelligibility is not well understood. The SII calculation procedures for the one-third octave band method and direct measurement of the input variables was used to assess improvements in speech intelligibility of the ANC systems (American National Standards Institute, 1998). The insertion gain of three ANC headsets and one external ANC system was measured. The attenuation for the external device was found to be $10 \mathrm{~dB}$ over a narrow frequency band. The open ear ANC headsets achieved comparable reduction at low frequency, but the band width of reduction was much broader. The open ear headsets also yielded high frequency attenuation due to their physical constructs. The closed ear ANC headset achieved impressive low frequency attenuation around $20 \mathrm{~dB}$ and high frequency attenuation of nearly $40 \mathrm{~dB}$. The NRR of the systems was calculated accounting for the transfer function data obtained for each device. The NCT Noise Buster Extreme NRR was found to be around 5 while the NCT ProActive NRR was 24. The remaining systems had NRRs around zero.

Traffic noise recorded at the toll plaza was used to calculate the noise spectrum for determination of the SII. For the current conditions at the toll plaza, the SII indicated that 
the environment was poor with respect to communication. Accounting for the effects of the ANC systems, the SII changed very little. The results suggest that the traffic noise level at the toll plaza overpowers the speech spectrum level and creates a low signal to noise ratio. Three different traffic scenarios were considered: average semi-truck traffic, idle semi-truck traffic, and idle car traffic. The idle vehicle states were believed to me the most representative of the actual communication conditions. The results for idle car noise suggest that passenger cars noise does not present a problem for speech intelligibility. Improved systems which increased the signal to noise ratio were modeled and tested. The results indicate that use of a sealed ANC headset with a dipole directional microphone yield a significant improvement in SII, as much as a 0.20 increase for vocal efforts which are common at the toll plaza. It was found that level overloading effects are more significant than low frequency masking. Results from and investigation into higher order microphone directivity demonstrated that a good communication system (a SII of 0.75 of above) is achievable for normal vocal effort.

\section{$\underline{6.2 \text { Recommendations }}$}

It is suggested that the possible existence of resonance conditions be investigated in a subsequent study. On-site measurements at many locations throughout the toll plaza could be used to investigate the presence of standing waves. With on-site measurements, the results of the beam tracing method could be validated.

Subsequent investigations into modified ANC headsets that improve the signal to noise ratio and thereby the speech intelligibility are suggested. Advanced concepts should integrate adaptive algorithms to achieve the project goals. Feedforward algorithms are adaptive and therefore behave differently for different input signals. As a starting point an adaptive algorithm may be placed between the directional microphone and the sealed ANC 
headset configuration as discussed in Chapter 5. If implemented properly, the filter could provide significant improvements in speech intelligibility by selectively attenuate traffic noise and preserving speech at a level that does not overload the ear. Using such a configuration the signal to noise ratio could be improved over the $6 \mathrm{~dB}$ increase due to a dipole directional microphones alone. Alternatively, the single channel feedback algorithm of existing ANC control headsets could be replaced by an adaptive ANC algorithm to yield similar results. Due to the adaptive nature of the systems, jury testing may be necessary to establish speech intelligibility performance. After an implementation has been identified which significantly improves the speech intelligibility at the toll plaza, testing of various embodiments with respect to comfort should be performed. 


\section{LIST OF REFERENCES}

American National Standards Institute. (1969). ANSI S3.5-1969: Methods For Calculation of The Articulation Index. New York: Acoustical Society of America.

American National Standards Institute. (1974). ANSI S3.19-1974: Method for the Measurement of Real-Ear Protection of Hearing Protectors and Physical Attenuation of Earmuffs. New York: Acoustical Society of America.

American National Standards Institute. (1977). ANSI S3.14-1977: For Rating Noise with Respect to Speech Interference. New York: Acoustical Society of America.

American National Standards Institute. (1995). ANSI S12.42-1995: Micropohone-InReal-Ear and Acoustic Test Fixture Methods for the Measurement of Insertion Loss of Circumaural HearingProtection Devices. New York: Acoustical Society of America.

American National Standards Institute. (1998). ANSI S3.5-1997: Methods For Calculation of The Speech Intelligibility Index. New York: Acoustical Society of America.

Arnold, D. (1998a). Winter Carbon Monoxide and Noise Surveys of Three Toll Booth Plazas: West Point, Portage, and East Point. Unpublished Indiana Department of Transportation Report.

Arnold, D. (1998b). Summer Carbon Monoxide and Noise Surveys of Three Toll Booth Plazas: West Point, Portage, and East Point. Unpublished Indiana Department of Transportation Report.

Beranek, L. L. (1996). Acoustics. Woodbury, NY: Acoustical Society of America .

Bies, D. A., \& Hansen, C. H. (1996). Engineering Noise Control: Theory And Practice (2nd ed.). New York: E \& FN Spon.

Born, M., \& Wolf, E. (1964). Principles of Optics: Electromagnetic Theory of Propagation, Interference and Diffraction of Light (2nd ed.). New York: The Macmillan Company.

Crocker, M. J. (1997a). Introduction. In M. J. Crocker (Ed.), Encyclopedia of Acoustics (pp. 3-19). New York: J. Wiley. 
Crocker, M. J. (1997b). Rating Measures, Descriptors, Criteria, and Procedures For Determining Human Response To Noise. In M. J. Crocker (Ed.), Encyclopedia of Acoustics (pp. 943-965). New York: J. Wiley.

Egan, J. P., \& Hake, H. W. (1950). On the Masking of a Simple Auditory Stimulus. The Journal of the Acoustical Society of America, 22(5), 622-630.

Fader, B. (1981). Industrial Noise Control. New York: J. Wiley.

Faulkner, L. L., (Ed.). (1976). Handbook of Industrial Noise Control. New York: Industrial Press.

Feist, J. P., Mongeau, L., \& Bernhard, R. J. (2001). Survey of Tollbooth Operators' Response to Traffic Noise and Performance of an Active Noise Control Headset. Transportation Research Record: Journal of the Transportation Research Board, 1756, 68-75.

Felsen, L. B. (1997). Ray Acoustics For Structures. In M. J. Crocker (Ed.), Encyclopedia of Acoustics (pp. 47-54). New York: J. Wiley.

Hawley, M. E. (Ed.). (1977). Speech Intelligibility and Speaker Recognition. Stroudsburg, PA: Dowden, Hutchington \& Ross.

Holt, J. G. (1990). Why Hi-Fi Experts Disagree. On Stereophile Test CD [CD]. Santa Fe, NM: Stereophile.

Houtgast, T., \& Steeneken, H. J. M. (1985). A Review of The MTF Concept in Room Acoustics And Its Use For Estimating Speech Intelligibility in Auditoria. Journal of The Acoustical Society of America, 77(3), 1069-1077.

Indiana Toll Road. (1982). Toll Plaza and Utility Building: Milepost 24 - Portage Mainland Barrier. Unpublished blueprints.

Irwin, J. D., \& Graf, E. R. (1979). Industrial Noise and Vibration Control. Englewood Cliffs, NJ: Prentice Hall.

Kinsler, Lawrence E., \& Frey Austin R. (1982). Fundamental of Acoustics (3rd ed.). New York: Wiley.

Kuttruff, H. (1997). Sound In Enclosures. In M. J. Crocker (Ed.), Encyclopedia of Acoustics (pp. 1101-1114). New York: J. Wiley.

LMS Numerical Technologies. (1998). RAYNOISE: Building Acoustics \& Industrial Noise Simulation (Revision 3.0) [Computer software and user's manual]. Leuvan, Belgium: LMS International.

Lunedburg, R. K. (1964). Mathematical Theory of Optics. Los Angeles: University of California Press.

Muradali, A., \& Fyfe, K. R. (1998). A Study of 2D and 3D Barrier Insertion Loss Using Improved Diffraction-Based Methods. Applied Acoustics, 53, 49-75. 
National Archives and Records Administration. (2001a, July). Title 29 of the Code of Federal Regulations, Part 1910, Section 95: Occupational Noise Exposure. Retrieved October 23, 2001 from The Code of Federal Regulations: via http://frwebgate.access.gpo.gov/cgi-bin/getcfr.cgi?TITLE=29\&PART $=1910 \&$ SECTION=95\&TYPE=PDF \&YEAR=2001

National Archives and Records Administration. (2001b, July). Title 40 of the Code of Federal Regulations, Part 211, Section 207: Computation of the Noise Reduction Rating (NRR). Retrieved October 23, 2001 from The Code of Federal Regulations: via http://frwebgate.access.gpo.gov/cgi-bin/getcfr.cgi?TITLE=40\&PART=211\&SECTION=207\&TYPE=PDF\&YEAR=2001

Nelson, P.A., \& Elliott, S.J. (1993). Active Control of Sound. San Diego, CA: Academic Press.

Nelson, P. A., \& Elliot, S. J. (1997). Active Noise Control. In M. J. Crocker (Ed.), Encyclopedia of Acoustics (pp. 1025-1037). New York: J. Wiley.

Snyder, S. D. (2000). Active Noise Control Primer. New York: Springer.

Tocci, G. C. (1997). Ratings and Descriptors For The Building Acoustical Environment. In M. J. Crocker (Ed.), Encyclopedia of Acoustics (pp. 1161-1179). New York: J. Wiley.

U. S. Department of Transportation. (1998). FHWA Traffic Noise Model (Version 1.0) [Computer software and technical manual]. Cambridge, MA: John A. Volpe National Transportation Systems Center.

Waters, D. E. (1988). An Investigation of Adaptive Digital Signal Processing Techniques to Active Noise Control. Unpublished master's thesis, Purdue University, West Lafayette, IN.

Waters, D. E., \& Bernhard, R. J. (1989). The Enhancement of Enclosure Performance Using Active Noise Control. In G. C. Maling (Ed.), Proceeding of Inter-Noise 89 (pp. 463-466). New York: Noise Control Foundation.

Weston, D. E. (1997). Ray Acoustics For Fluids. In M. J. Crocker (Ed.), Encyclopedia of Acoustics (pp. 39-45). New York: J. Wiley.

Ziomek, L. J. (1985). Underwater Acoustics: A Linear Systems Theory Approach. Orlando, FL: Academic Press. 
APPENDICES 


\section{Appendix A: Toll Road Employee Surveys}

\section{A.1 Survey 1 and Results}

This section includes the first survey distributed to toll road employees and the results. 


\section{Noise Survey}

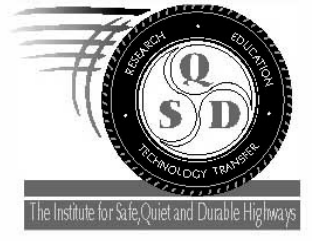

The Institute for Safe, Quiet, and Durable Highways

Herrick Laboratories

Purdue University - West Lafayette Campus

1. How old are you?
a) 16 - 25 years
b) 26 - 35 years
c) 36 - 45 years
d) 46 - 55 years
e) $55+$ years

2. How long have you worked regularly in a tollbooth?
a) 0 - 6 months
b) 7 - 12 months
c) 1 - 2 years
d) 2 - 5 years
e) $5+$ years

3. Do you have known hearing loss?
a) Yes
b) No

4. Where do you spend the majority of your time while working? (If you work in multiple positions, please select the position you work at the most and answer the rest of the questions with that position in mind.)
a) Body entirely in a toll-booth
b) Body partially in a toll-booth
c) Body entirely outside of a toll-booth, but still near the road
d) Most time spent outdoors away from the tollbooths
e) Most time spent indoor, but not in a tollbooth

5. How long are the work shifts during which you are exposed to traffic noise?
a) $0-2$ Hours
b) 4 Hours
c) 6 Hours
d) 8 Hours
e) More than 8 Hours

6. With respect to noise, how would you consider the conditions under which you normally work ?
a) Very Poor
b) Poor
c) Satisfactory
d) Good
e) Excellent

7. This type of vehicle's noise emissions is annoying. (For each of the blanks below write the letter a, b, c, d, or e that corresponds to your answer.)
a) Strongly Agree
b) Agree
c) Undecided
d) Disagree
e) Strongly Disagree

- Semi-Trucks

Braking
- Braking
B Braking
B Braking

Accelerating
- Accelerating
- Accelerating
Accelerating
Idling Idling

- Buses and Delivery Trucks - Motorcycles — Braking

8. Please indicate any other type of vehicle noise that you find annoying.

9. Do you get noise induced headaches at work?
a) Very Frequently
b) Frequently
c) Occasionally
d) Rarely
e) Very Rarely
f) Never

10. Do you get headaches while not at work?
a) Very Frequently
b) Frequently
c) Occasionally
d) Rarely
e) Very Rarely
f) Never

11. With respect to noise at work, do your ears often feel "tired" after work?
a) Very Frequently
b) Frequently
c) Occasionally
d) Rarely
e) Very Rarely
f) Never 
12. As a result of background noise, do you often find it difficult to communicate with drivers?
a) Very Frequently
b) Frequently
c) Occasionally
d) Rarely
e) Very Rarely
f) Never

13. What other noise related problems do you encounter at the toll plaza?

14. Would you object to the idea of communicating with drivers through a speaker?
a) Yes
b) No

Any suggestions you have that might improve the noise conditions in your workplace would be graciously accepted. You are more than welcome to comment on any noise factors not considered in this survey that you feel need attention.

Please feel free to use the space below. 
Subject No.

\begin{tabular}{|c|c|c|c|c|c|c|c|c|c|c|c|}
\hline 1 & $\mathrm{c}$ & $\mathrm{e}$ & $\mathrm{a}$ & $\mathrm{a}$ & $\mathrm{e}$ & $\mathrm{a}$ & $\mathrm{a}$ & $\mathrm{c}$ & $\mathrm{a}$ & $\mathrm{a}$ & $\mathrm{b}$ \\
\hline 2 & $\mathrm{~b}$ & $\mathrm{~d}$ & $\mathrm{a}$ & $\mathrm{a}$ & $\mathrm{d}$ & $\mathrm{a}$ & $\mathrm{c}$ & $\mathrm{c}$ & c & $\mathrm{b}$ & $\mathrm{b}$ \\
\hline 3 & $\mathrm{c}$ & $\mathrm{d}$ & $\mathrm{a}$ & $\mathrm{e}$ & $\mathrm{e}$ & $\mathrm{a}$ & $\mathrm{c}$ & $\mathrm{c}$ & $\mathrm{b}$ & $\mathrm{a}$ & $\mathrm{a}$ \\
\hline 4 & $\mathrm{a}$ & $\mathrm{d}$ & $\mathrm{a}$ & $\mathrm{b}$ & $\mathrm{e}$ & $\mathrm{b}$ & $\mathrm{e}$ & $\mathrm{d}$ & $\mathrm{c}$ & & \\
\hline 5 & $\mathrm{~d}$ & $\mathrm{~b}$ & $\mathrm{~b}$ & $\mathrm{a}$ & $\mathrm{d}$ & $\mathrm{d}$ & $\mathrm{f}$ & $\mathrm{c}$ & $\mathrm{c}$ & $\mathrm{b}$ & $\mathrm{b}$ \\
\hline 6 & $\mathrm{~d}$ & $\mathrm{~d}$ & b & $\mathrm{a}$ & $\mathrm{e}$ & $\mathrm{c}$ & c & d & c & $\mathrm{a}$ & $\mathrm{a}$ \\
\hline 7 & $\mathrm{c}$ & $\mathrm{a}$ & $\mathrm{b}$ & $\mathrm{a}$ & $\mathrm{d}$ & $\mathrm{c}$ & $\mathrm{c}$ & $\mathrm{e}$ & $\mathrm{d}$ & $\mathrm{a}$ & $\mathrm{b}$ \\
\hline 8 & $\mathrm{e}$ & $\mathrm{e}$ & $\mathrm{b}$ & $\mathrm{a}$ & $\mathrm{d}$ & $\mathrm{c}$ & $\mathrm{c}$ & $\mathrm{e}$ & $\mathrm{c}$ & $\mathrm{b}$ & $\mathrm{a}$ \\
\hline 9 & $\mathrm{~d}$ & $\mathrm{~b}$ & $\mathrm{~b}$ & $\mathrm{a}$ & $\mathrm{e}$ & $\mathrm{a}$ & $\mathrm{c}$ & $\mathrm{e}$ & & $\mathrm{a}$ & $\mathrm{b}$ \\
\hline 10 & $\mathrm{~d}$ & $\mathrm{e}$ & $\mathrm{b}$ & $\mathrm{b}$ & $\mathrm{e}$ & $\mathrm{a}$ & $\mathrm{b}$ & $\mathrm{b}$ & $\mathrm{b}$ & $\mathrm{a}$ & $\mathrm{a}$ \\
\hline 11 & $\mathrm{~b}$ & $\mathrm{a}$ & b & b & $\mathrm{e}$ & $\mathrm{b}$ & $\mathrm{c}$ & $\mathrm{c}$ & $\mathrm{c}$ & $\mathrm{a}$ & $\mathrm{a}$ \\
\hline 12 & $\mathrm{e}$ & $\mathrm{b}$ & $\mathrm{b}$ & $\mathrm{a}$ & $\mathrm{d}$ & $\mathrm{c}$ & $\mathrm{f}$ & $\mathrm{f}$ & $\mathrm{f}$ & $\mathrm{a}$ & $\mathrm{a}$ \\
\hline 13 & $\mathrm{a}$ & $\mathrm{b}$ & $\mathrm{b}$ & $\mathrm{b}$ & $\mathrm{d}$ & $\mathrm{c}$ & $\mathrm{c}$ & $\mathrm{d}$ & $\mathrm{c}$ & $\mathrm{b}$ & $\mathrm{b}$ \\
\hline 14 & $\mathrm{c}$ & $\mathrm{c}$ & $\mathrm{b}$ & $\mathrm{a}$ & $\mathrm{e}$ & $\mathrm{b}$ & $\mathrm{f}$ & $\mathrm{d}$ & $\mathrm{f}$ & c & $\mathrm{a}$ \\
\hline 15 & $\mathrm{c}$ & $\mathrm{c}$ & b & $\mathrm{a}$ & $\mathrm{d}$ & $\mathrm{c}$ & $\mathrm{f}$ & $\mathrm{c}$ & $\mathrm{e}$ & $\mathrm{c}$ & $\mathrm{a}$ \\
\hline 16 & $\mathrm{c}$ & $\mathrm{e}$ & $\mathrm{b}$ & $\mathrm{a}$ & $\mathrm{e}$ & $\mathrm{a}$ & $\mathrm{a}$ & $\mathrm{c}$ & $\mathrm{b}$ & $\mathrm{a}$ & $\mathrm{b}$ \\
\hline 17 & $\mathrm{c}$ & $\mathrm{a}$ & $\mathrm{b}$ & $\mathrm{a}$ & $\mathrm{d}$ & $\mathrm{a}$ & $\mathrm{f}$ & $\mathrm{f}$ & $\mathrm{e}$ & $\mathrm{a}$ & $\mathrm{b}$ \\
\hline 18 & $\mathrm{e}$ & $\mathrm{e}$ & $\mathrm{b}$ & $\mathrm{a}$ & $\mathrm{e}$ & $\mathrm{b}$ & $\mathrm{d}$ & $\mathrm{d}$ & $\mathrm{b}$ & $\mathrm{a}$ & $\mathrm{a}$ \\
\hline 19 & $\mathrm{~d}$ & $\mathrm{e}$ & $\mathrm{b}$ & $\mathrm{a}$ & $\mathrm{d}$ & $\mathrm{b}$ & $\mathrm{c}$ & $\mathrm{d}$ & $\mathrm{b}$ & $\mathrm{a}$ & $\mathrm{b}$ \\
\hline 20 & $\mathrm{c}$ & $\mathrm{b}$ & $\mathrm{b}$ & $\mathrm{a}$ & $\mathrm{d}$ & $\mathrm{c}$ & $\mathrm{e}$ & $\mathrm{e}$ & $\mathrm{f}$ & $\mathrm{b}$ & $\mathrm{a}$ \\
\hline 21 & $\mathrm{~d}$ & $\mathrm{~b}$ & $\mathrm{~b}$ & $\mathrm{a}$ & $\mathrm{e}$ & $\mathrm{b}$ & $\mathrm{d}$ & $\mathrm{e}$ & $\mathrm{d}$ & $\mathrm{a}$ & $\mathrm{b}$ \\
\hline 22 & $\mathrm{c}$ & $\mathrm{b}$ & $\mathrm{b}$ & $\mathrm{b}$ & $\mathrm{e}$ & $\mathrm{c}$ & $\mathrm{c}$ & $\mathrm{d}$ & $\mathrm{c}$ & $\mathrm{a}$ & $\mathrm{b}$ \\
\hline 23 & $\mathrm{~d}$ & $\mathrm{e}$ & $\mathrm{b}$ & $\mathrm{b}$ & $\mathrm{d}$ & $\mathrm{b}$ & $\mathrm{c}$ & $\mathrm{d}$ & $\mathrm{b}$ & $\mathrm{a}$ & $\mathrm{a}$ \\
\hline 24 & $\mathrm{~d}$ & $\mathrm{e}$ & $\mathrm{b}$ & $\mathrm{b}$ & $\mathrm{d}$ & $\mathrm{b}$ & $\mathrm{c}$ & $\mathrm{d}$ & $\mathrm{b}$ & $\mathrm{b}$ & $\mathrm{b}$ \\
\hline 25 & $\mathrm{e}$ & $\mathrm{e}$ & $\mathrm{b}$ & $\mathrm{a}$ & $\mathrm{e}$ & $\mathrm{b}$ & $\mathrm{e}$ & $\mathrm{e}$ & $\mathrm{c}$ & $\mathrm{b}$ & $\mathrm{a}$ \\
\hline 26 & $\mathrm{a}$ & $\mathrm{c}$ & $\mathrm{b}$ & $\mathrm{a}$ & $\mathrm{e}$ & $\mathrm{b}$ & $\mathrm{b}$ & $\mathrm{c}$ & $\mathrm{a}$ & $\mathrm{a}$ & $\mathrm{b}$ \\
\hline 27 & $\mathrm{c}$ & $\mathrm{a}$ & $\mathrm{b}$ & $\mathrm{b}$ & $\mathrm{d}, \mathrm{e}$ & $\mathrm{b}$ & $\mathrm{c}$ & $\mathrm{d}$ & $\mathrm{c}$ & $\mathrm{a}$ & $\mathrm{b}$ \\
\hline 28 & $\mathrm{~d}$ & $\mathrm{e}$ & $\mathrm{b}$ & $\mathrm{b}$ & $\mathrm{b}, \mathrm{d}$ & $\mathrm{b}$ & $\mathrm{b}$ & $\mathrm{c}$ & $\mathrm{c}$ & $\mathrm{b}$ & $\mathrm{b}$ \\
\hline 29 & $\mathrm{~d}$ & $\mathrm{a}$ & $\mathrm{b}$ & $\mathrm{a}$ & $\mathrm{d}$ & $\mathrm{c}$ & $\mathrm{d}$ & $\mathrm{d}$ & $\mathrm{c}$ & $\mathrm{b}$ & $\mathrm{b}$ \\
\hline
\end{tabular}

\begin{tabular}{|c|c|c|c|c|c|c|c|c|c|c|c|}
\hline Respo & & & & & & & & & & & \\
\hline a & 3 & 5 & 4 & 19 & 0 & 7 & 2 & 0 & 2 & 17 & 12 \\
\hline b & 2 & 7 & 25 & 9 & 0 & 12 & 3 & 1 & 7 & 9 & 16 \\
\hline $\mathrm{c}$ & 10 & 3 & 0 & 0 & 0 & 9 & 13 & 9 & 12 & 2 & 0 \\
\hline d & 10 & 4 & 0 & 0 & 13 & 1 & 3 & 11 & 2 & 0 & 0 \\
\hline $\mathrm{e}$ & 4 & 10 & 0 & 1 & 14 & 0 & 3 & 6 & 2 & 0 & 0 \\
\hline f & 0 & 0 & 0 & 0 & 0 & 0 & 5 & 2 & 3 & 0 & 0 \\
\hline
\end{tabular}

\begin{tabular}{|l|l|l|l|l|l|l|l|l|l|l|l|} 
Total & 29 & 29 & 29 & 29 & 27 & 29 & 29 & 29 & 28 & 28 & 28 \\
\hline
\end{tabular}

Table A.1: Results for Survey 1. 


\begin{tabular}{|c|c|c|c|c|c|c|c|c|c|c|c|c|}
\hline $\begin{array}{c}\text { Subject } \\
\text { No }\end{array}$ & $\begin{array}{c}\text { S.T. } \\
\text { Brake }\end{array}$ & $\begin{array}{c}\text { S.T. } \\
\text { Accel }\end{array}$ & $\begin{array}{l}\text { S.T. } \\
\text { Idle }\end{array}$ & $\begin{array}{l}\text { Buses } \\
\text { Brake }\end{array}$ & $\begin{array}{l}\text { Buses } \\
\text { Accel }\end{array}$ & $\begin{array}{c}\text { Buses } \\
\text { Idle }\end{array}$ & $\begin{array}{l}\text { Cars } \\
\text { Brake } \\
\end{array}$ & $\begin{array}{l}\text { Cars } \\
\text { Accel }\end{array}$ & $\begin{array}{c}\text { Cars } \\
\text { Idle }\end{array}$ & $\begin{array}{c}\text { M.C. } \\
\text { Brake }\end{array}$ & $\begin{array}{c}\text { M.C. } \\
\text { Accel }\end{array}$ & $\begin{array}{l}\text { M.C } \\
\text { Idle }\end{array}$ \\
\hline 1 & $\mathrm{a}$ & $\mathrm{a}$ & $\mathrm{a}$ & $\mathrm{a}$ & $\mathrm{a}$ & $\mathrm{a}$ & & $b$ & $b$ & & $\mathrm{a}$ & $\mathrm{a}$ \\
\hline 2 & $\mathrm{a}$ & $\mathrm{a}$ & $\mathrm{a}$ & $\mathrm{d}$ & $\mathrm{d}$ & d & $\mathrm{d}$ & $\mathrm{d}$ & $\mathrm{d}$ & $b$ & d & $b$ \\
\hline 3 & $\mathrm{a}$ & $\mathrm{a}$ & $\mathrm{a}$ & $b$ & $\mathrm{a}$ & $\mathrm{a}$ & $\mathrm{a}$ & $\mathrm{a}$ & $a$ & $a$ & $\mathrm{a}$ & $\mathrm{a}$ \\
\hline 4 & $b$ & $\mathrm{a}$ & $b$ & $b$ & $\mathrm{a}$ & $b$ & $\mathrm{~d}$ & $\mathrm{~d}$ & $\mathrm{~d}$ & $\mathrm{a}$ & $\mathrm{a}$ & $\mathrm{a}$ \\
\hline 5 & $b$ & $\mathrm{~b}$ & $C$ & $b$ & $b$ & $b$ & $b$ & $\mathrm{a}$ & $\mathrm{C}$ & $\mathrm{a}$ & $\mathrm{a}$ & $b$ \\
\hline 6 & $b$ & $\mathrm{C}$ & $\mathrm{C}$ & $\mathrm{C}$ & $\mathrm{a}$ & $\mathrm{C}$ & $b$ & $b$ & $\mathrm{C}$ & $\mathrm{a}$ & $\mathrm{a}$ & $b$ \\
\hline 7 & $b$ & $\mathrm{a}$ & $b$ & $c$ & $b$ & $C$ & $\mathrm{~d}$ & $\mathrm{~d}$ & $\mathrm{~d}$ & $C$ & $\mathrm{a}$ & $\mathrm{a}$ \\
\hline 8 & $\mathrm{~d}$ & $b$ & $b$ & $b$ & $b$ & $b$ & $\mathrm{~d}$ & $\mathrm{~d}$ & $\mathrm{~d}$ & $\mathrm{a}$ & $\mathrm{a}$ & $\mathrm{a}$ \\
\hline 9 & $\mathrm{a}$ & $\mathrm{a}$ & & & & & & & & & & \\
\hline 10 & $\mathrm{a}$ & $\mathrm{a}$ & $\mathrm{a}$ & $b$ & $\mathrm{a}$ & & & & & $\mathrm{a}$ & $\mathrm{a}$ & $\mathrm{a}$ \\
\hline 11 & $a$ & $a$ & $a$ & $b$ & $a$ & $a$ & $b$ & $b$ & $b$ & $b$ & $a$ & $\mathrm{a}$ \\
\hline 12 & $b$ & $\mathrm{C}$ & $\mathrm{d}$ & $\mathrm{d}$ & $\mathrm{d}$ & $\mathrm{d}$ & $\mathrm{e}$ & $\mathrm{e}$ & $\mathrm{e}$ & $d$ & $\mathrm{a}$ & $\mathrm{d}$ \\
\hline 13 & $b$ & $\mathrm{a}$ & $\mathrm{a}$ & $b$ & $\mathrm{a}$ & $b$ & $\mathrm{C}$ & $b$ & $\mathrm{C}$ & C & $\mathrm{a}$ & $c$ \\
\hline 14 & a & $b$ & C & a & $b$ & C & $a$ & $b$ & C & $a$ & a & $a$ \\
\hline 15 & $c$ & $C$ & $C$ & $\mathrm{C}$ & $\mathrm{C}$ & $\mathrm{C}$ & $\mathrm{C}$ & $C$ & $\mathrm{C}$ & $c$ & $c$ & $\mathrm{C}$ \\
\hline 16 & $\mathrm{a}$ & $\mathrm{a}$ & $\mathrm{a}$ & $\mathrm{a}$ & $\mathrm{a}$ & $\mathrm{a}$ & $\mathrm{d}$ & $\mathrm{d}$ & $\mathrm{d}$ & $d$ & $b$ & $\mathrm{~d}$ \\
\hline 17 & $\mathrm{a}$ & $\mathrm{a}$ & $\mathrm{a}$ & $b$ & $b$ & $b$ & $b$ & $b$ & $\mathrm{C}$ & $\mathrm{a}$ & $\mathrm{a}$ & $\mathrm{a}$ \\
\hline 18 & $\mathrm{a}$ & $\mathrm{a}$ & $\mathrm{a}$ & $\mathrm{a}$ & $\mathrm{a}$ & $\mathrm{a}$ & & $\mathrm{a}$ & $a$ & & $\mathrm{a}$ & $\mathrm{a}$ \\
\hline 19 & $\mathrm{a}$ & $\mathrm{a}$ & $\mathrm{a}$ & $\mathrm{a}$ & $\mathrm{a}$ & $\mathrm{a}$ & $C$ & $C$ & $\mathrm{C}$ & $b$ & $\mathrm{a}$ & $\mathrm{a}$ \\
\hline 20 & & & $\mathrm{e}$ & & & $\mathrm{e}$ & & & $\mathrm{e}$ & & $\mathrm{e}$ & \\
\hline 21 & $\mathrm{a}$ & $\mathrm{a}$ & $\mathrm{d}$ & $\mathrm{a}$ & $\mathrm{a}$ & $\mathrm{a}$ & $b$ & $b$ & $b$ & $\mathrm{~d}$ & $\mathrm{a}$ & $\mathrm{a}$ \\
\hline 22 & $c$ & $\mathrm{a}$ & $b$ & $\mathrm{~d}$ & $\mathrm{~d}$ & $\mathrm{~d}$ & $b$ & $b$ & $b$ & a & $\mathrm{a}$ & $\mathrm{a}$ \\
\hline 23 & $b$ & $b$ & $b$ & $b$ & $\mathrm{a}$ & $C$ & $C$ & $C$ & $\mathrm{C}$ & C & $b$ & C \\
\hline 24 & $b$ & $b$ & $b$ & & $\mathrm{a}$ & $c$ & $c$ & $b$ & $b$ & $\mathrm{~d}$ & $b$ & $\mathrm{~b}$ \\
\hline 25 & $\mathrm{a}$ & & & a & $\mathrm{a}$ & & $\mathrm{a}$ & $\mathrm{a}$ & & a & & $\mathrm{a}$ \\
\hline 26 & $\mathrm{a}$ & $\mathrm{d}$ & $c$ & $\mathrm{~d}$ & $\mathrm{a}$ & $b$ & $\mathrm{e}$ & $\mathrm{d}$ & $\mathrm{C}$ & $\mathrm{a}$ & $\mathrm{a}$ & $\mathrm{a}$ \\
\hline 27 & $\mathrm{a}$ & $\mathrm{a}$ & $\mathrm{a}$ & $\mathrm{C}$ & & & $\mathrm{C}$ & & & $\mathrm{a}$ & $\mathrm{a}$ & $\mathrm{a}$ \\
\hline 28 & $a$ & $\mathrm{a}$ & $b$ & $b$ & a & $C$ & $b$ & $\mathrm{a}$ & $\mathrm{d}$ & $b$ & a & C \\
\hline 29 & $b$ & $\mathrm{a}$ & $C$ & $\mathrm{C}$ & $b$ & $\mathrm{e}$ & $\mathrm{d}$ & $\mathrm{d}$ & $b$ & $\mathrm{e}$ & $\mathrm{e}$ & $\mathrm{e}$ \\
\hline
\end{tabular}

\begin{tabular}{|c|c|c|c|c|c|c|c|c|c|c|c|c|}
\hline Response & \multicolumn{10}{|c|}{} \\
\hline $\mathrm{a}$ & 16 & 18 & 11 & 7 & 16 & 7 & 3 & 5 & 2 & 12 & 20 & 16 \\
\hline $\mathrm{b}$ & 9 & 5 & 7 & 10 & 6 & 6 & 7 & 9 & 6 & 4 & 3 & 4 \\
\hline $\mathrm{c}$ & 2 & 3 & 6 & 5 & 1 & 7 & 6 & 3 & 9 & 4 & 1 & 4 \\
\hline $\mathrm{d}$ & 1 & 1 & 2 & 4 & 3 & 3 & 6 & 7 & 6 & 4 & 1 & 2 \\
\hline $\mathrm{e}$ & 0 & 0 & 1 & 0 & 0 & 2 & 2 & 1 & 2 & 1 & 2 & 1 \\
\hline
\end{tabular}

\begin{tabular}{|l|l|l|l|l|l|l|l|l|l|l|l|l|} 
Total & 28 & 27 & 27 & 26 & 26 & 25 & 24 & 25 & 25 & 25 & 27 & 27 \\
\hline
\end{tabular}

Table A.2: Results for Question 7, Survey 1. 


\section{Written responses to Question 8, Survey 1}

- Horns (trucks and cars)

- Air Brakes releasing

- Semi tire blow out

- Jake Brakes

- Load trucks and motor cycles

- Straight Pipes

- Un-muffled dual exhaust especially on pick-up trucks

- Snow plows scraping pavement in winter

- All types

- Bus traffic is extremely loud and the exhaust is horrible

- Glass-pack mufflers

- Dual exhausts

\section{Written responses to Question 13, Survey 1}

- Difficult to hear intercom and Road Radio

- Road Maintenance should have a different frequency (especially in the winter)

- Car radios

- Car horns under canopy

- Potholes

- Non business communication and idle chatter between toll attendants on the intercom system

- Phone

Any suggestions you have that might improve the noise conditions in your workplace would be graciously accepted. You are more than welcome to comment on any noise factors not considered in this survey that you feel need attention.

- Working through a window versus fully open door

- Maintenance should have a there own intercom

- Sound proofing booth and making them completely enclosed

- Headset was great but too awkward to wear for a period of time with cord. Patrons also thought that I was listening to a radio instead of paying attention to them. Would like head set if small and cord was not in the way.

- Vehicle horns from impatient drivers should be prohibited. Extreme acceleration of motorcycles should not be allowed. Also sound proofing the booths would be a great help. 


\section{A.2 Survey 2 and Results}

This section includes the second survey distributed to toll road employees and the results. 


\section{Noise Survey - follow up}

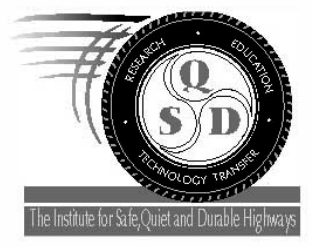

The Institute for Safe, Quiet, and Durable Highways

Herrick Laboratories

Purdue University - West Lafayette Campus

This survey should be administer to employees who have completed the first "Noise Survey" and were able to use the NCT Inc. Noise Buster Extreme active noise control headphones. Please circle the answer that agrees with your selection.

1. How long did you use the active noise control headphones?
a) $0-2$ Hours
b) 4 Hours
c) 6 Hours
d) 8 Hours
e) More than 8 Hours

2. In terms of reducing traffic/road noise annoyance, please rate the effectiveness of the headphones.
a) Excellent
b) Above Average
c) Average
d) Below Average
e) Extremely Poor

3. The overall working environment was more pleasant with the headphones on.
a) Strongly Agree
b) Agree
c) Undecided
d) Disagree
e) Strongly Disagree

4. The headphones improved my ability to communicate with customers.
a) Strongly Agree
b) Agree
c) Undecided
d) Disagree
e) Strongly Disagree

5. The headphones were comfortable to wear, and I am willing to use them throughout the work day.
a) Strongly Agree
b) Agree
c) Undecided
d) Disagree
e) Strongly Disagree

6. Neglecting any comfort or discomfort, I was impressed with the performance of the headphones.
a) Strongly Agree
b) Agree
c) Undecided
d) Disagree
e) Strongly Disagree

7. The headphones you used might have given the appearance that you were listening to a personal stereo. Would you object to wearing cordless headphones that were just as effective but looked like a communication headset? (Perhaps the headphones would contain a microphone).
a) Yes, I would object
b) No, I would not object

8. How old are you?
a) 16 - 25 years
b) 26 - 35 years
c) $36-45$ years
d) 46 - 55 years
e) $55+$ years

9. How long have you worked regularly in a toll booth?
a) 0 - 6 months
b) 7 - 12 months
c) 1 - 2 years
d) 2 - 5 years
e) $5+$ years

10. Do you have known hearing loss?
a) Yes
b) $\mathrm{No}$

11. How long are the work shifts during which you are exposed to traffic noise?
a) $0-2$ Hours
b) 4 Hours
c) 6 Hours
d) 8 Hours
e) More than 8 Hours

12. Please rate the overall value of the active noise control headphones in the work environment.
a) Excellent
b) Above Average
c) Average
d) Below Average
e) Extremely Poor

Please feel free to use the space below or the back side of this sheet to provide additional comments concerning the headphones. 
Subject

\begin{tabular}{|l|l|l|l|l|l|l|l|l|l|l|l|}
\hline \multicolumn{10}{|c|}{ Question No. } \\
\hline 1 & 2 & 3 & 4 & 5 & 6 & 7 & 8 & 9 & 10 & 11 & 12 \\
\hline
\end{tabular}

\begin{tabular}{|c|c|c|c|c|c|c|c|c|c|c|c|c|}
\hline 1 & $a$ & $d$ & $\mathrm{e}$ & $\mathrm{e}$ & $\mathrm{e}$ & e & $a$ & $d$ & $\mathrm{e}$ & $a$ & d & $\mathrm{e}$ \\
\hline 2 & $\mathrm{C}$ & $\mathrm{b}$ & $\mathrm{b}$ & $\mathrm{b}$ & $\mathrm{b}$ & $\mathrm{b}$ & $\mathrm{b}$ & $\mathrm{C}$ & $b$ & $\mathrm{~b}$ & $\mathrm{~d}$ & $b$ \\
\hline 3 & $a$ & $\mathrm{C}$ & $\mathrm{b}$ & $\mathrm{e}$ & & $\mathrm{b}$ & $\mathrm{a}$ & $d$ & $\mathrm{e}$ & $\mathrm{b}$ & e & $\mathrm{e}$ \\
\hline 4 & $a$ & $b$ & $\mathrm{C}$ & $c$ & $\mathrm{~d}$ & $\mathrm{~b}$ & $b$ & $d$ & d & $b$ & $d$ & C \\
\hline 5 & $\mathrm{~b}$ & $\mathrm{C}$ & $\mathrm{b}$ & $\mathrm{d}$ & $\mathrm{b}$ & $\mathrm{b}$ & $b$ & & $\mathrm{e}$ & $b$ & $d$ & C \\
\hline 6 & $\mathrm{C}$ & $\mathrm{a}$ & $a$ & $\mathrm{a}$ & $a$ & $a$ & $\mathrm{~b}$ & $\mathrm{C}$ & $a$ & $\mathrm{~b}$ & $\mathrm{~d}, \mathrm{e}$ & a \\
\hline 7 & $b$ & $b$ & $\mathrm{C}$ & $b$ & $\mathrm{C}$ & $\mathrm{b}$ & $b$ & $\mathrm{~d}$ & $b$ & $b$ & $d$ & $b$ \\
\hline 8 & $\mathrm{a}$ & $\mathrm{a}$ & $\mathrm{a}$ & $b$ & $\mathrm{~b}$ & $\mathrm{a}$ & $b$ & $\mathrm{a}$ & $b$ & $b$ & $d$ & $\mathrm{a}$ \\
\hline 9 & $a$ & $\mathrm{~b}$ & $\mathrm{~b}$ & c & $a$ & $\mathrm{~b}$ & $\mathrm{~b}$ & $\mathrm{~d}$ & $\mathrm{e}$ & $b$ & d & $b$ \\
\hline 10 & $\mathrm{a}$ & $\mathrm{b}$ & $\mathrm{b}$ & $b$ & $\mathrm{~d}$ & $\mathrm{~b}$ & $\mathrm{a}$ & $\mathrm{C}$ & $\mathrm{e}$ & $\mathrm{b}$ & $\mathrm{e}$ & $b$ \\
\hline 11 & $\mathrm{a}$ & $b$ & $\mathrm{C}$ & $c$ & $\mathrm{C}$ & $\mathrm{b}$ & $\mathrm{b}$ & $\mathrm{C}$ & $b$ & $\mathrm{~b}$ & $\mathrm{~d}, \mathrm{e}$ & $b$ \\
\hline 12 & $\mathrm{a}$ & $\mathrm{C}$ & $\mathrm{C}$ & $\mathrm{e}$ & $\mathrm{e}$ & $\mathrm{C}$ & $\mathrm{b}$ & $d$ & $\mathrm{e}$ & $\mathrm{b}$ & $\mathrm{e}$ & $c$ \\
\hline 13 & $\mathrm{~b}$ & $\mathrm{a}$ & $\mathrm{b}$ & $\mathrm{a}$ & $\mathrm{c}$ & $\mathrm{a}$ & $\mathrm{b}$ & $\mathrm{C}$ & $\mathrm{e}$ & $\mathrm{a}$ & $\mathrm{e}$ & $\mathrm{a}$ \\
\hline 14 & $b$ & $\mathrm{C}$ & $\mathrm{d}$ & $\mathrm{d}$ & $\mathrm{d}$ & $\mathrm{C}$ & $\mathrm{a}$ & $\mathrm{d}$ & $\mathrm{a}$ & $\mathrm{b}$ & d & C \\
\hline 15 & $\mathrm{~d}$ & $\mathrm{C}$ & $\mathrm{b}$ & $c$ & $\mathrm{e}$ & $\mathrm{b}$ & $\mathrm{b}$ & $\mathrm{a}$ & $\mathrm{a}$ & $\mathrm{b}$ & $\mathrm{d}, \mathrm{e}$ & $b$ \\
\hline 16 & $\mathrm{a}$ & $\mathrm{C}$ & $\mathrm{C}$ & $c$ & $\mathrm{~d}$ & $\mathrm{C}$ & $\mathrm{a}$ & $\mathrm{C}$ & $\mathrm{C}$ & $\mathrm{b}$ & $d$ & $c$ \\
\hline 17 & $\mathrm{a}$ & $\mathrm{a}$ & $\mathrm{a}$ & $b$ & $\mathrm{C}$ & $\mathrm{a}$ & $\mathrm{b}$ & $\mathrm{C}$ & $\mathrm{a}$ & $\mathrm{b}$ & $d$ & $\mathrm{a}$ \\
\hline 18 & $\mathrm{a}$ & $\mathrm{b}$ & $\mathrm{b}$ & $b$ & $\mathrm{e}$ & $\mathrm{b}$ & $\mathrm{b}$ & $\mathrm{C}$ & $\mathrm{e}$ & $\mathrm{b}$ & $d$ & $b$ \\
\hline 19 & $a$ & $\mathrm{C}$ & $\mathrm{e}$ & $\mathrm{e}$ & $\mathrm{e}$ & $\mathrm{C}$ & $b$ & $b$ & $\mathrm{a}$ & $b$ & $d$ & $b$ \\
\hline 20 & $\mathrm{a}$ & $\mathrm{C}$ & $\mathrm{C}$ & d & $\mathrm{e}$ & $\mathrm{C}$ & $\mathrm{a}$ & $\mathrm{C}$ & $b$ & $\mathrm{~b}$ & $d$ & $\mathrm{C}$ \\
\hline 21 & $a$ & $\mathrm{~d}$ & $\mathrm{~d}$ & d & $\mathrm{d}$ & $\mathrm{d}$ & $\mathrm{a}$ & $\mathrm{e}$ & $\mathrm{e}$ & $\mathrm{b}$ & $d$ & $\mathrm{~d}$ \\
\hline 22 & $\mathrm{a}$ & $\mathrm{d}$ & $\mathrm{d}$ & $c$ & $\mathrm{~d}$ & $\mathrm{~d}$ & $b$ & $\mathrm{e}$ & $b$ & $b$ & $d$ & $\mathrm{~d}$ \\
\hline 23 & $\mathrm{C}$ & $\mathrm{b}$ & $\mathrm{C}$ & d & $d$ & $\mathrm{C}$ & $\mathrm{b}$ & $d$ & $\mathrm{e}$ & $b$ & $\mathrm{e}$ & $\mathrm{C}$ \\
\hline 24 & $\mathrm{~d}$ & $\mathrm{a}$ & $\mathrm{a}$ & $\mathrm{d}$ & $\mathrm{b}$ & $\mathrm{b}$ & $\mathrm{b}$ & $d$ & $b$ & $\mathrm{~b}$ & $\mathrm{e}$ & $\mathrm{a}$ \\
\hline 25 & $a$ & $b$ & $\mathrm{~d}$ & $\mathrm{~d}$ & $\mathrm{e}$ & $b$ & $\mathrm{a}$ & $\mathrm{C}$ & d & $b$ & $d$ & $\mathrm{C}$ \\
\hline 26 & $\mathrm{a}$ & $\mathrm{C}$ & $\mathrm{b}$ & d & $\mathrm{d}$ & $\mathrm{b}$ & $\mathrm{a}$ & C & $\mathrm{d}$ & $b$ & $\mathrm{~d}$ & $\mathrm{C}$ \\
\hline 27 & $\mathrm{a}$ & $\mathrm{C}$ & $\mathrm{b}$ & $c$ & $\mathrm{~d}$ & $\mathrm{~b}$ & $\mathrm{a}$ & $\mathrm{C}$ & $\mathrm{C}$ & $\mathrm{b}$ & $d$ & $\mathrm{C}$ \\
\hline 28 & $\mathrm{~d}$ & $\mathrm{~b}$ & $\mathrm{~b}$ & $b$ & $\mathrm{~d}$ & $\mathrm{~b}$ & $\mathrm{~b}$ & $b$ & d & $\mathrm{a}$ & $d$ & $b$ \\
\hline 29 & $a$ & $\mathrm{C}$ & $\mathrm{C}$ & $\mathrm{b}$ & $\mathrm{C}$ & $\mathrm{b}$ & $\mathrm{b}$ & $d$ & $\mathrm{e}$ & $a$ & d & C \\
\hline
\end{tabular}

Response

\begin{tabular}{|c|c|c|c|c|c|c|c|c|c|c|c|c|}
\hline $\mathrm{a}$ & 19 & 5 & 4 & 2 & 2 & 4 & 10 & 2 & 5 & 4 & 0 & 5 \\
\hline $\mathrm{b}$ & 4 & 10 & 11 & 8 & 4 & 16 & 19 & 2 & 7 & 25 & 0 & 9 \\
\hline $\mathrm{c}$ & 3 & 11 & 8 & 7 & 5 & 6 & 0 & 12 & 2 & 0 & 0 & 11 \\
\hline $\mathrm{d}$ & 3 & 3 & 4 & 8 & 10 & 2 & 0 & 10 & 4 & 0 & 20 & 2 \\
\hline $\mathrm{e}$ & 0 & 0 & 2 & 4 & 7 & 1 & 0 & 2 & 11 & 0 & 6 & 2 \\
\hline
\end{tabular}

\begin{tabular}{|l|l|l|l|l|l|l|l|l|l|l|l|l|}
\hline Total & 29 & 29 & 29 & 29 & 28 & 29 & 29 & 28 & 29 & 29 & 26 & 29 \\
\hline
\end{tabular}

Table A.3: Results for Survey 2. 
Please feel free to use the space below or the back side of this sheet to provide additional comments concerning the headphones.

- Patrons were rude because they thought I was using a stereo system

- In regards to question \#5, the headphones weren't uncomfortable, they would just take a getting use to

- The headphones blocked the road noise but also made it hard to hear anyone speaking to you

- Couldn't hear patrons...s sounded like they were in the distance. I don't like things over my ears (irritating)

- Headphones hurt with glasses on 
Appendix B: Toll Plaza Beam Tracing Model

\section{B.1 Model Diagram}

The toll plaza and various noise control solutions were modeled using a beam tracing method. The schematic of the model is shown in Figure B.1. The figure includes the source location and number in addition to lane position. This schematic will serve as a key to the following figures presented in Appendix B. 


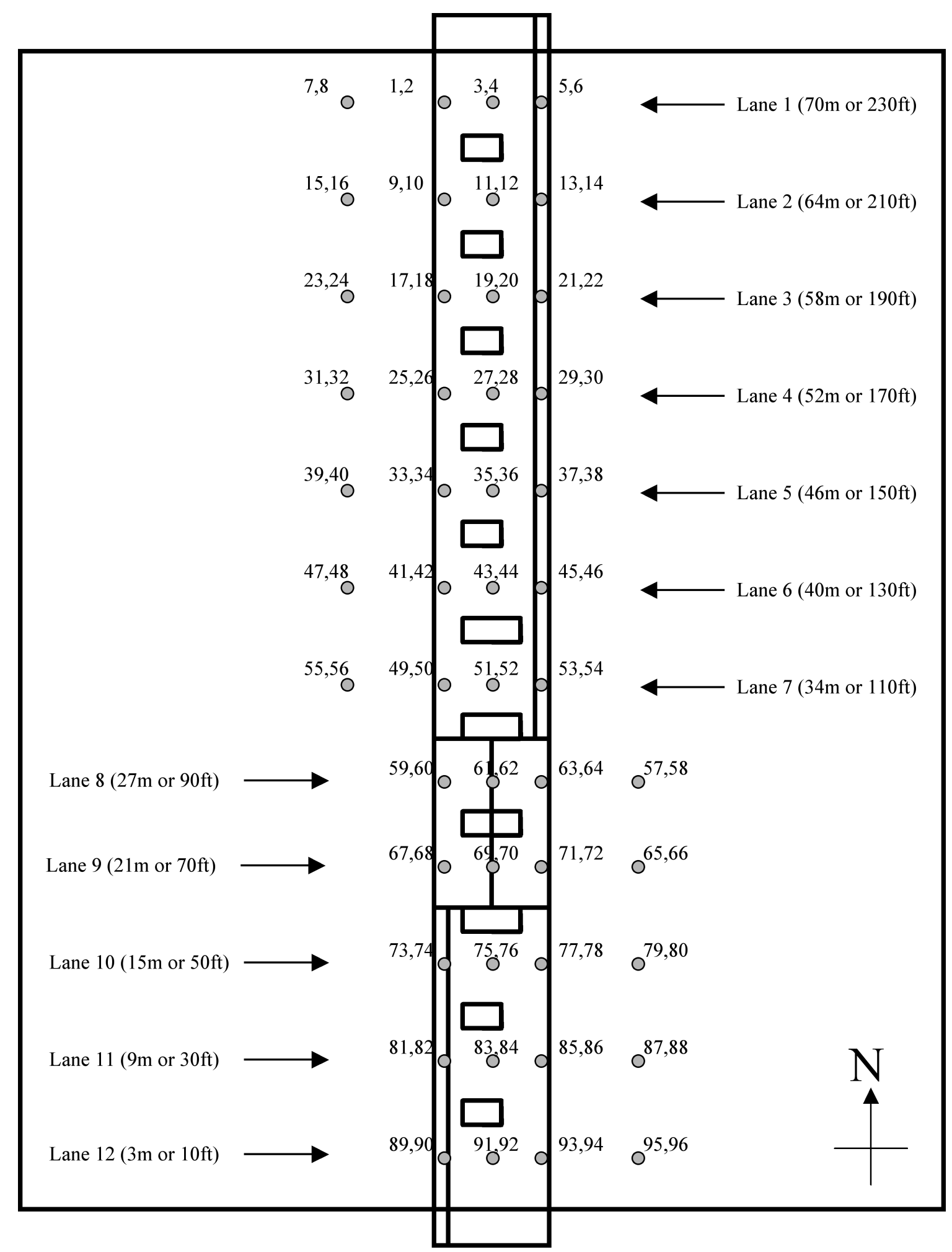

Figure B.1: Schematic of the toll plaza model. 


\section{B.2 Frequency Dependence of Passive Noise Control Solutions}

The figure in this section display the frequency dependence of the noise control solutions evaluated in the beam tracing model of the toll plaza. All semi-truck sources were activated. Each figure shows the response at a particular window location. 


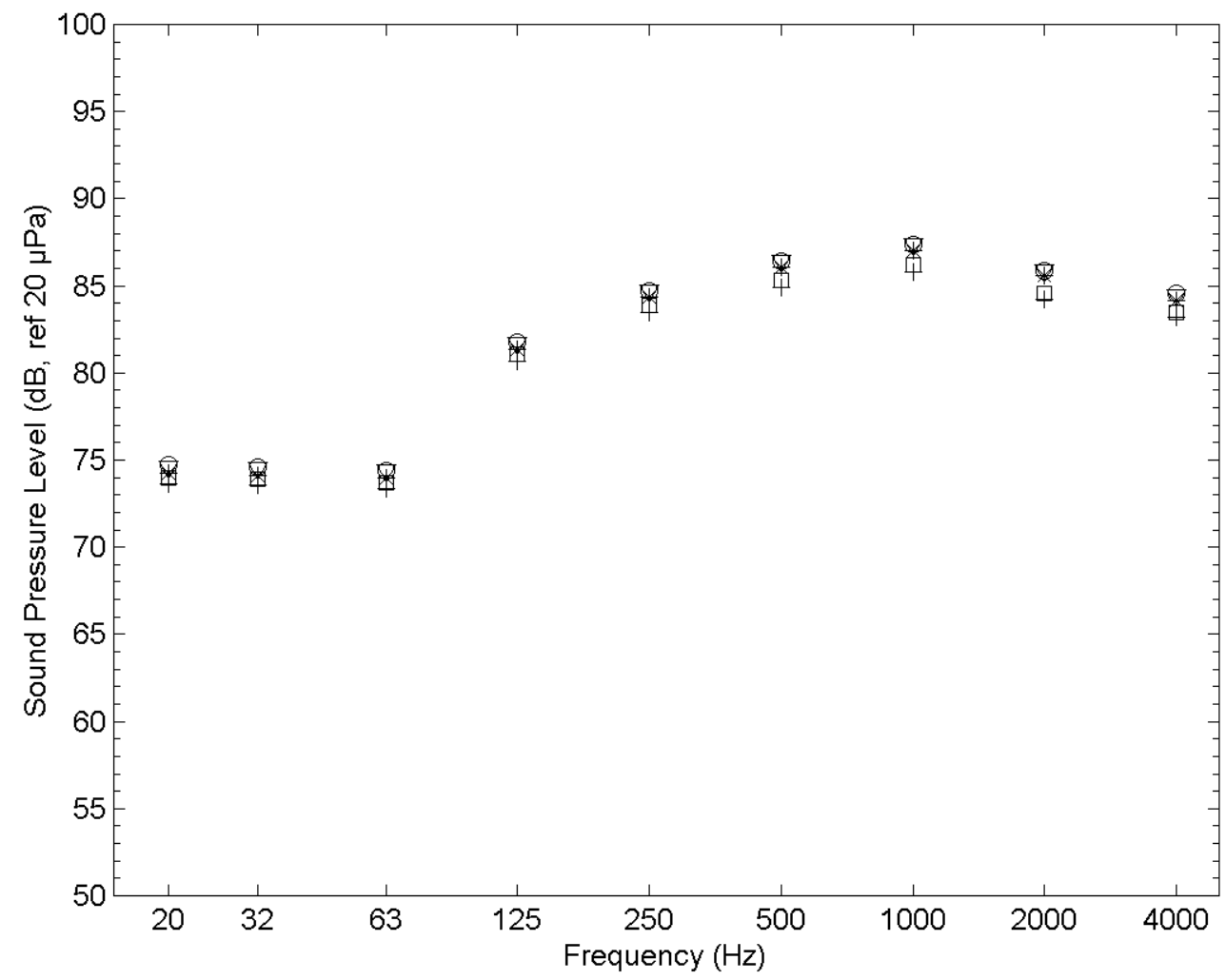

Figure B.2: $\quad$ Response at window location of 68 meters (window south of lane 1), all semi-truck sources active. $\nabla$ level of existing toll plaza; * level with absorbing canopy; O level with absorbing barriers; level with absorbing barriers; + level with all modifications. 


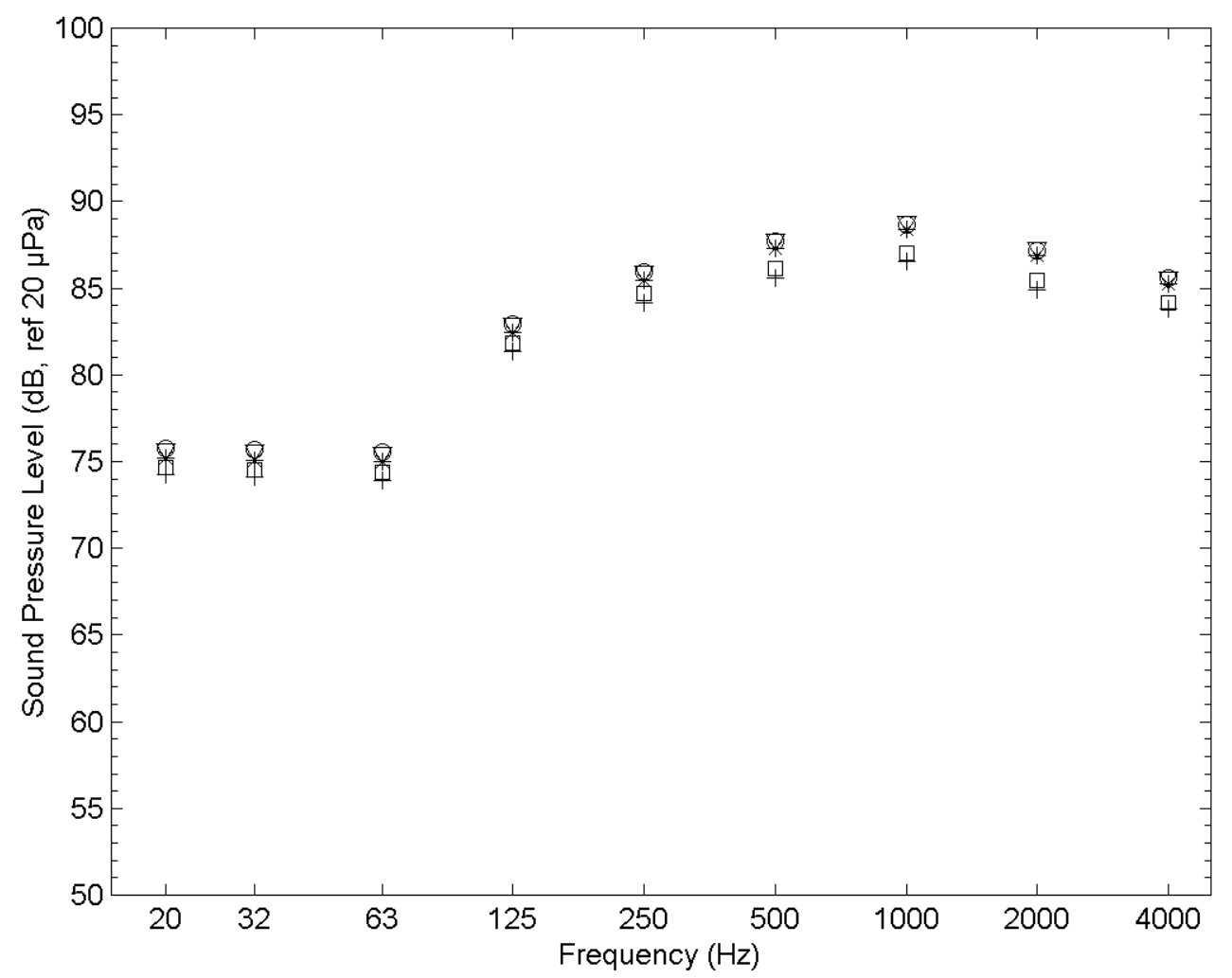

Figure B.3: $\quad$ Response at window location of 62 meters (window south of lane 2), all semi-truck sources active. $\nabla$ level of existing toll plaza; ${ }^{*}$ level with absorbing canopy; O level with absorbing barriers; level with absorbing barriers; + level with all modifications. 


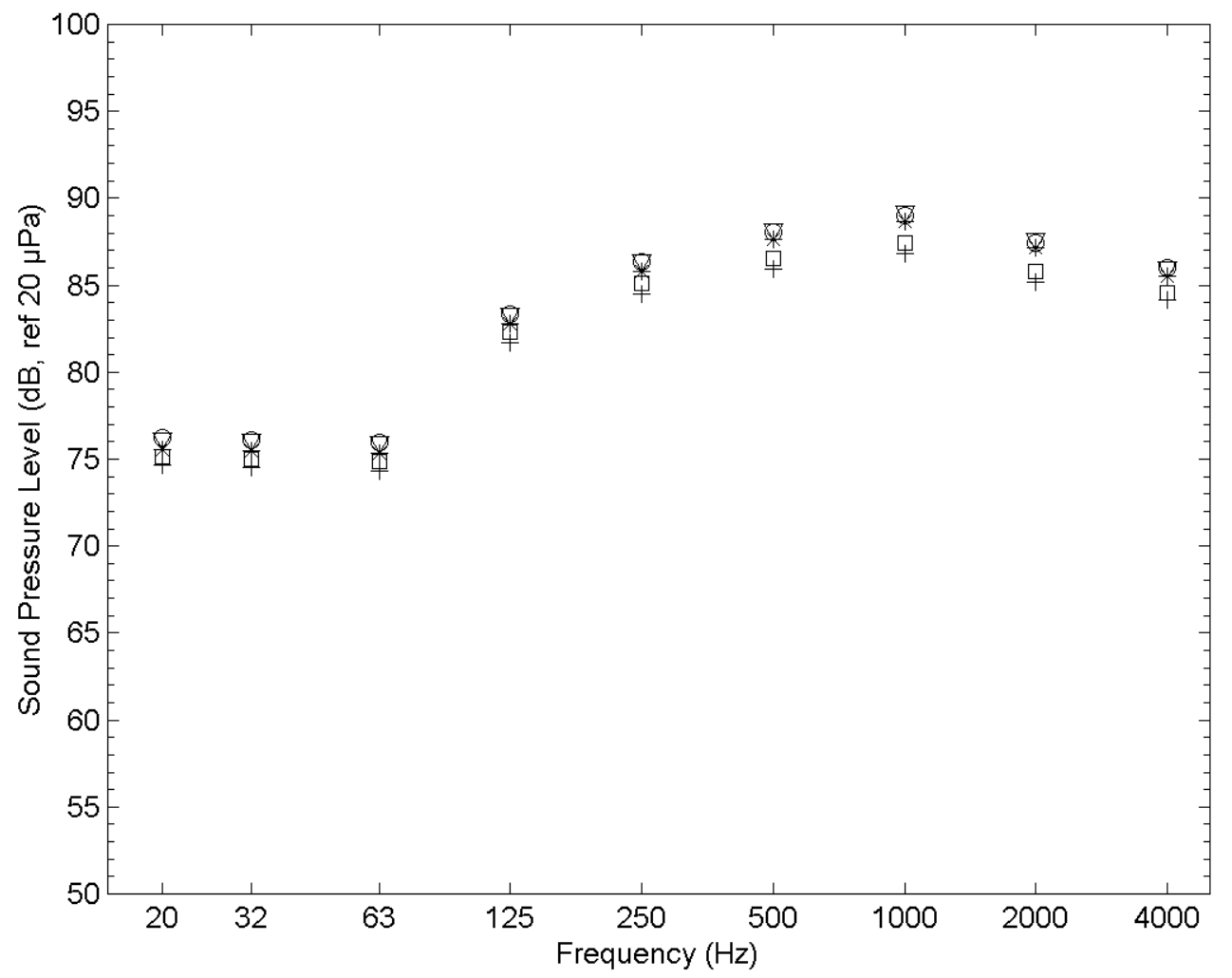

Figure B.4: $\quad$ Response at window location of 56 meters (window south of lane 3), all semi-truck sources active. $\nabla$ level of existing toll plaza; ${ }^{*}$ level with absorbing canopy; O level with absorbing barriers; level with absorbing barriers; + level with all modifications. 


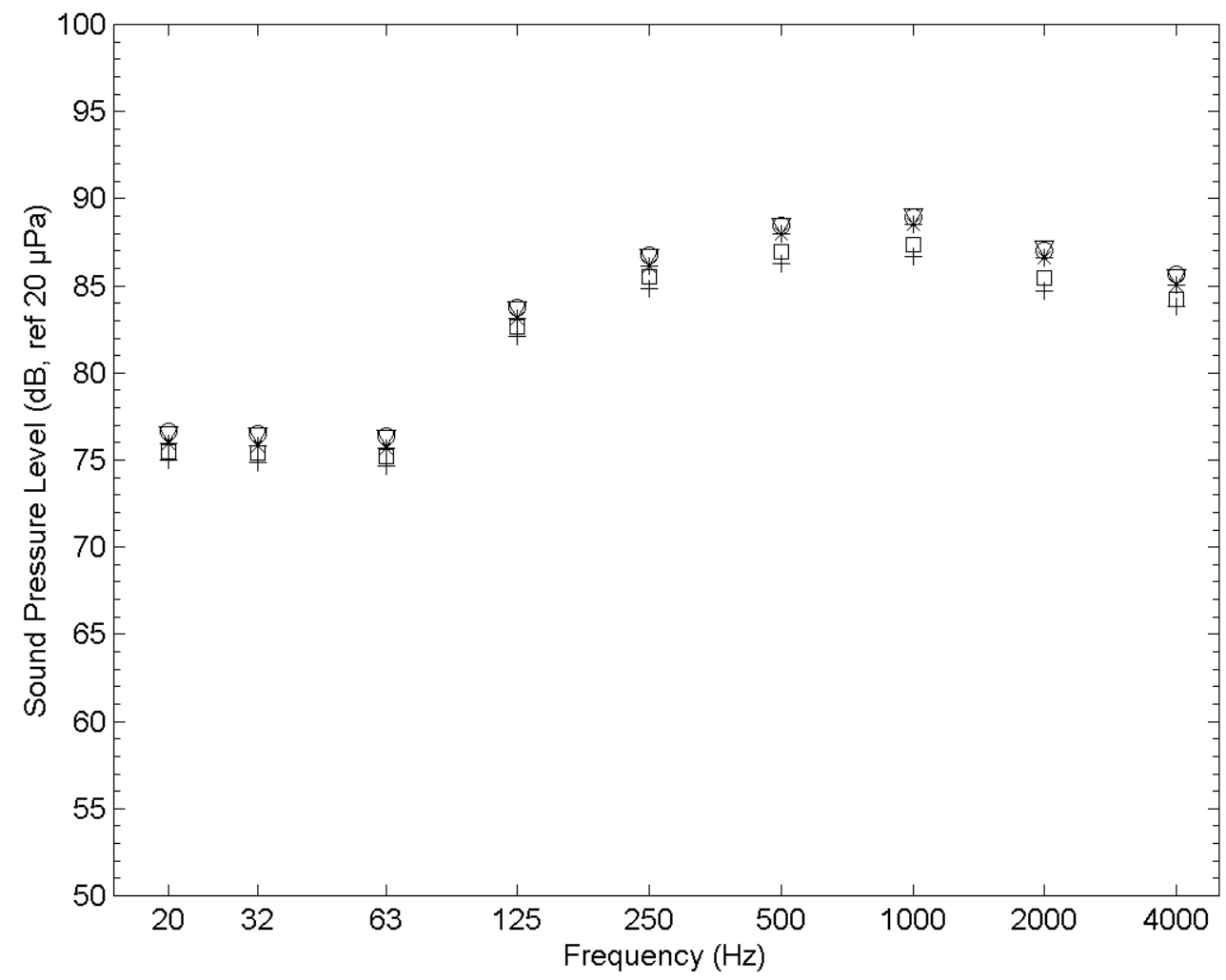

Figure B.5: $\quad$ Response at window location of 50 meters (window south of lane 4), all semi-truck sources active. $\nabla$ level of existing toll plaza; * level with absorbing canopy; O level with absorbing barriers; level with absorbing barriers; + level with all modifications. 


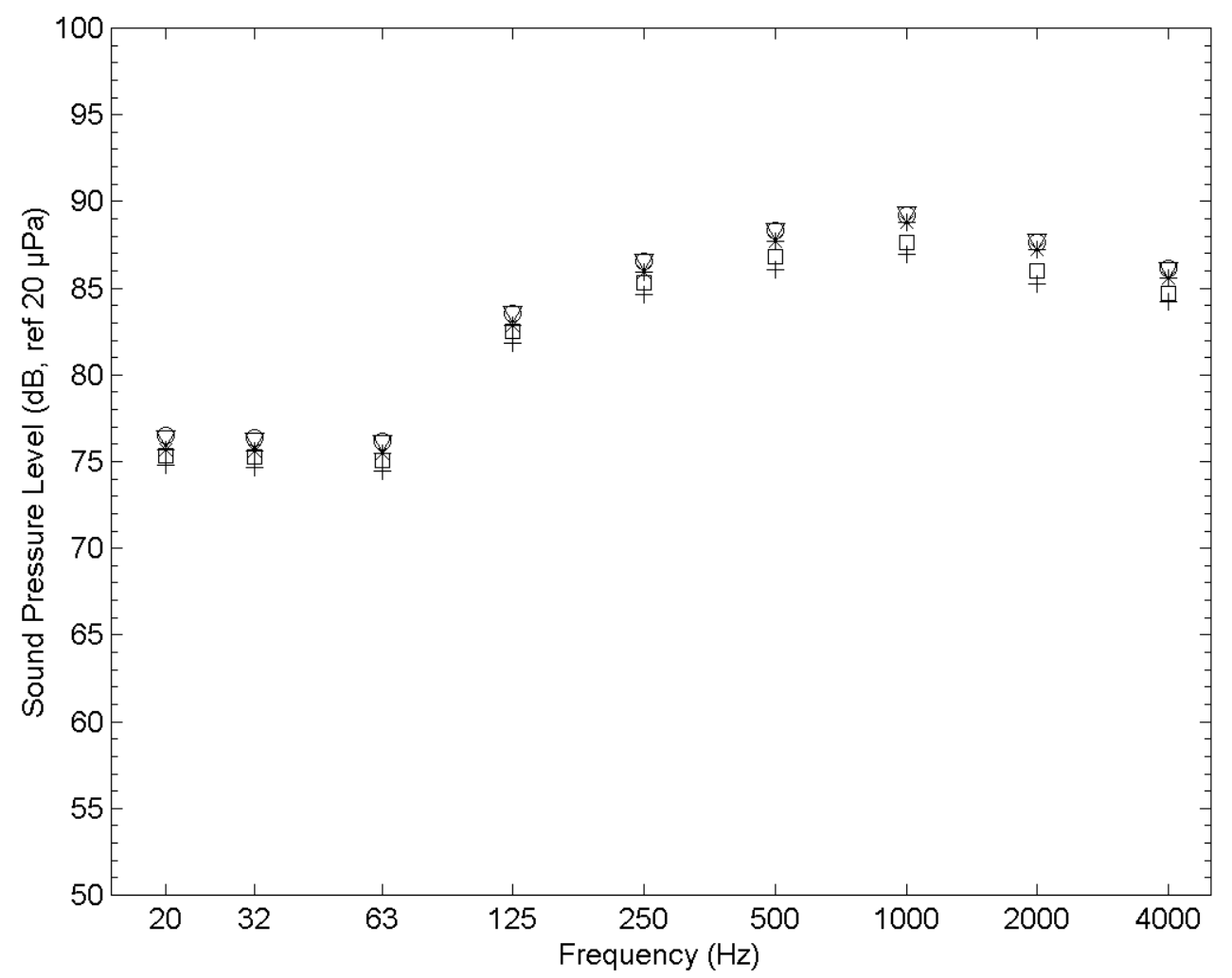

Figure B.6: Response at window location of 44 meters (window south of lane 5), all semi-truck sources active. $\nabla$ level of existing toll plaza; ${ }^{*}$ level with absorbing canopy; O level with absorbing barriers; level with absorbing barriers; + level with all modifications. 


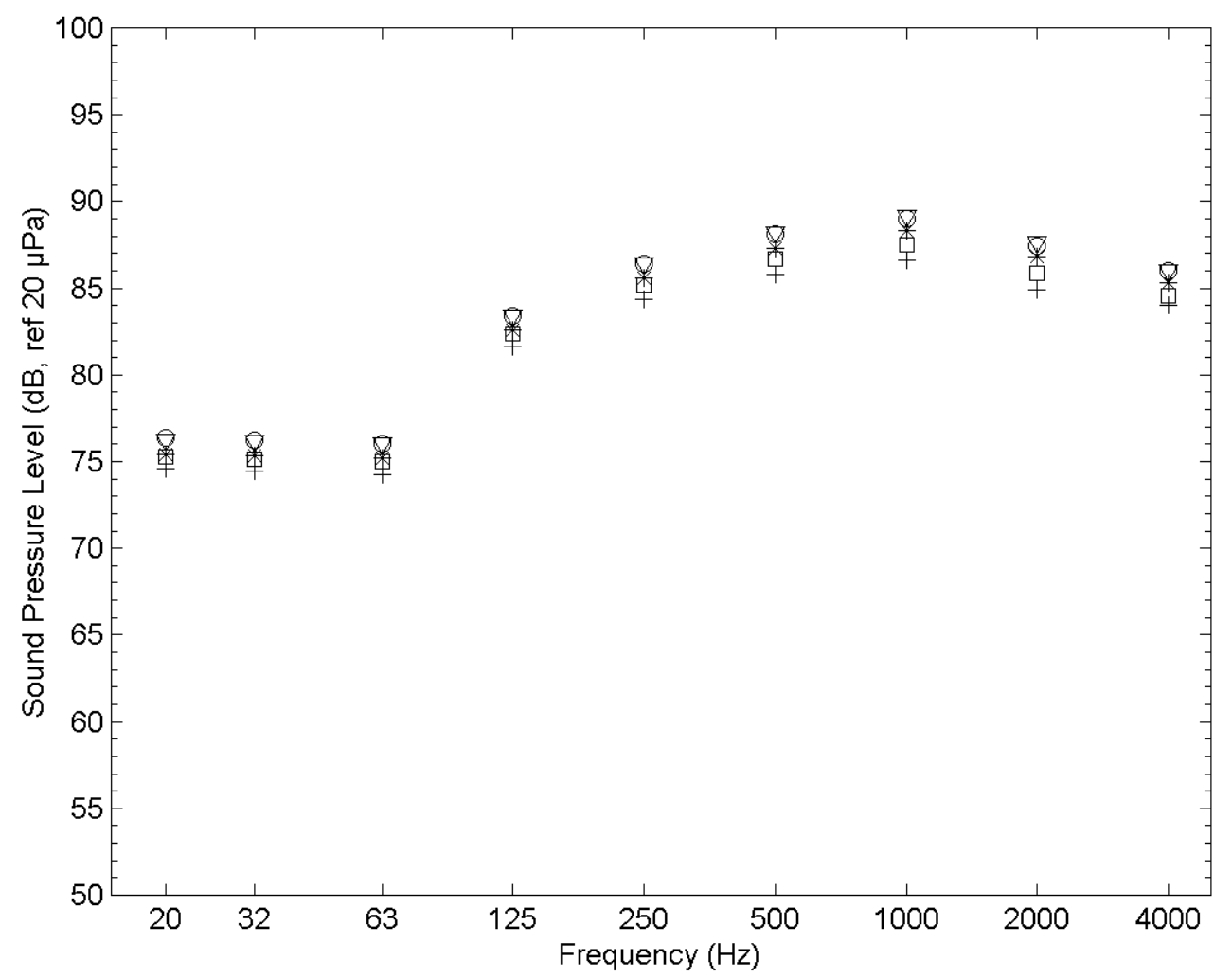

Figure B.7: $\quad$ Response at window location of 37 meters (window south of lane 6), all semi-truck sources active. $\nabla$ level of existing toll plaza; * level with absorbing canopy; O level with absorbing barriers; level with absorbing barriers; + level with all modifications. 


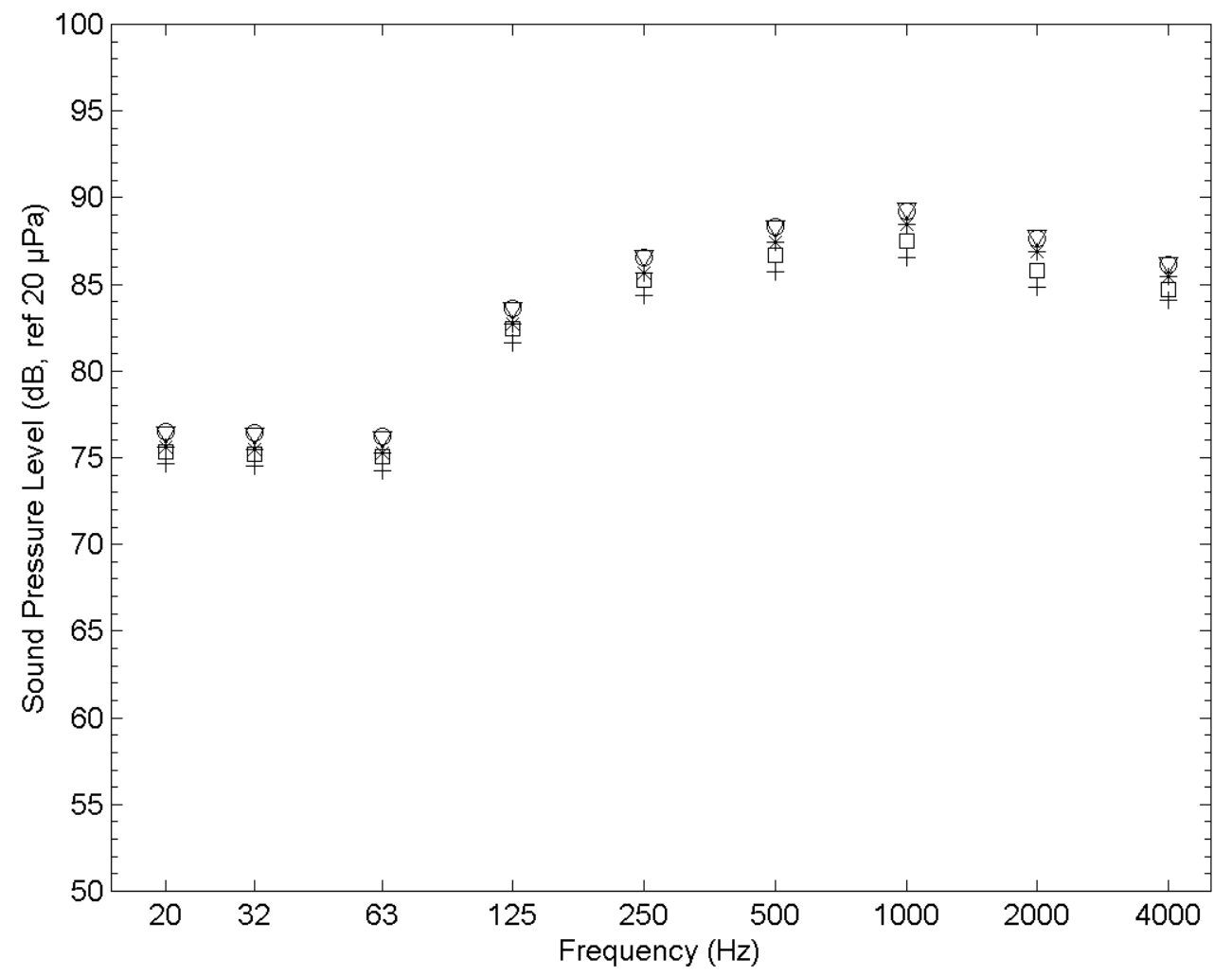

Figure B.8: $\quad$ Response at window location of 31 meters (window south of lane 7), all semi-truck sources active. $\nabla$ level of existing toll plaza; * level with absorbing canopy; O level with absorbing barriers; level with absorbing barriers; + level with all modifications. 


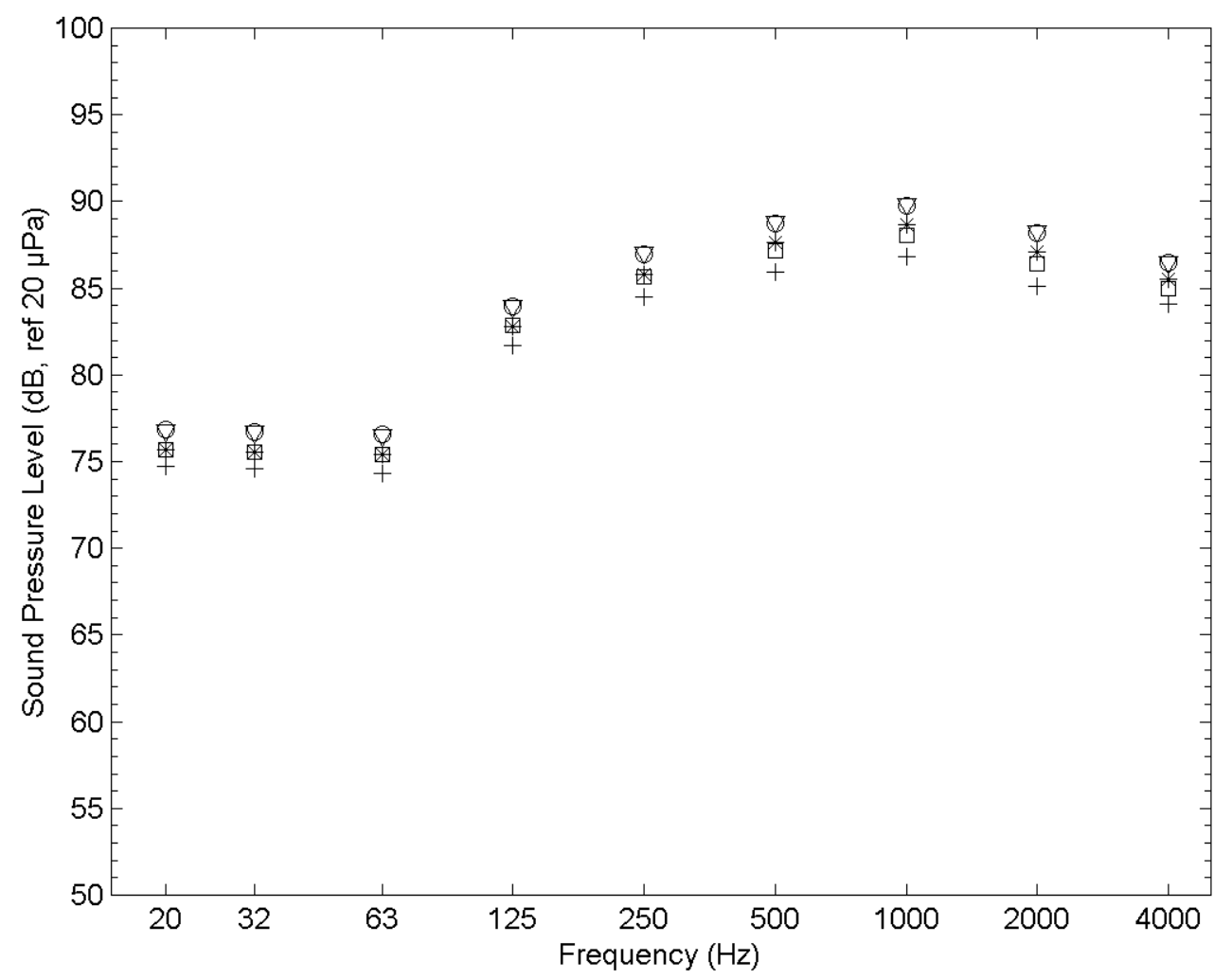

Figure B.9: $\quad$ Response at window location of 25 meters (window south of lane 8), all semi-truck sources active. $\nabla$ level of existing toll plaza; ${ }^{*}$ level with absorbing canopy; O level with absorbing barriers; level with absorbing barriers; + level with all modifications. 


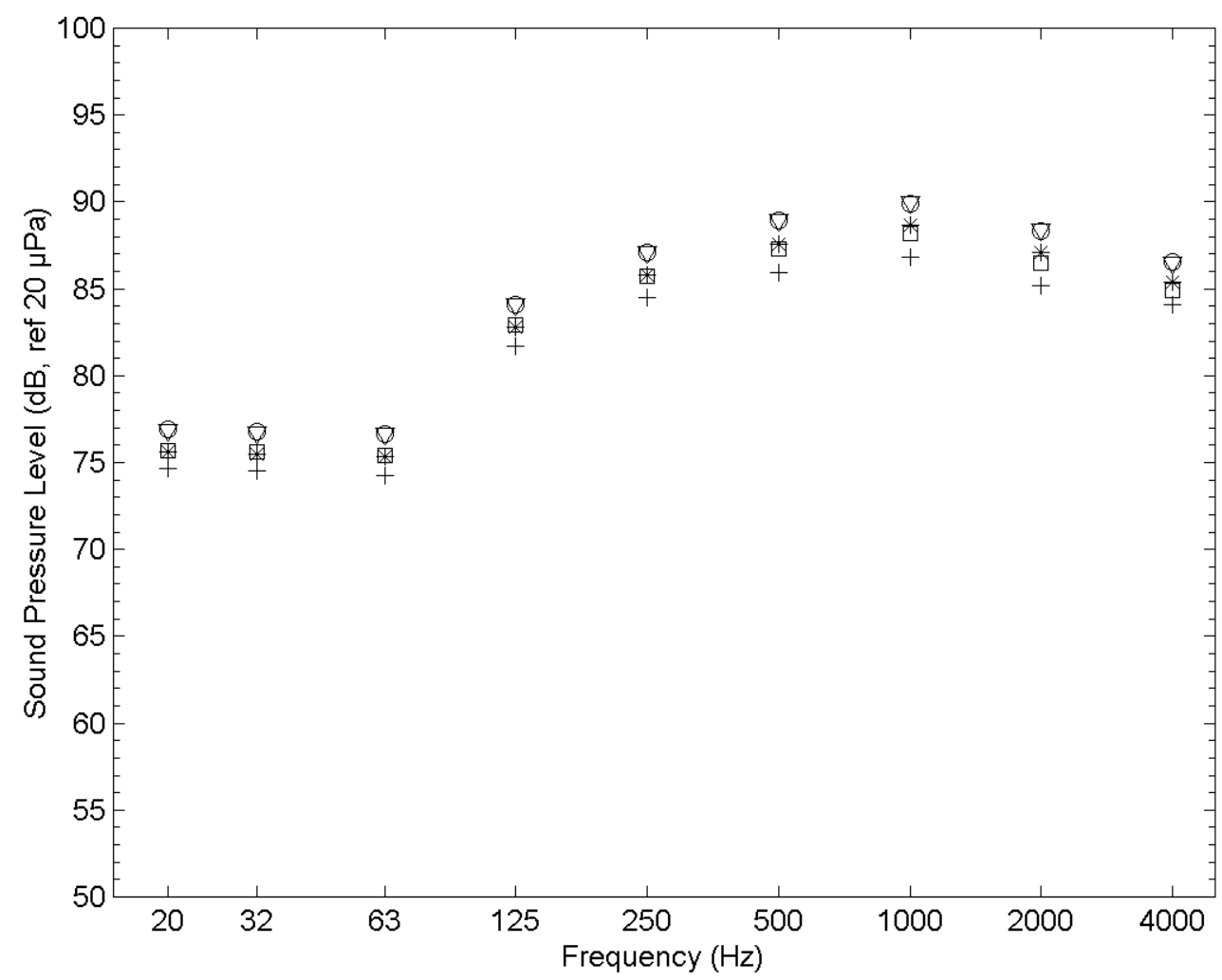

Figure B.10: Response at window location of 19 meters (window south of lane 9), all semi-truck sources active. $\nabla$ level of existing toll plaza; * level with absorbing canopy; O level with absorbing barriers; level with absorbing barriers; + level with all modifications. 


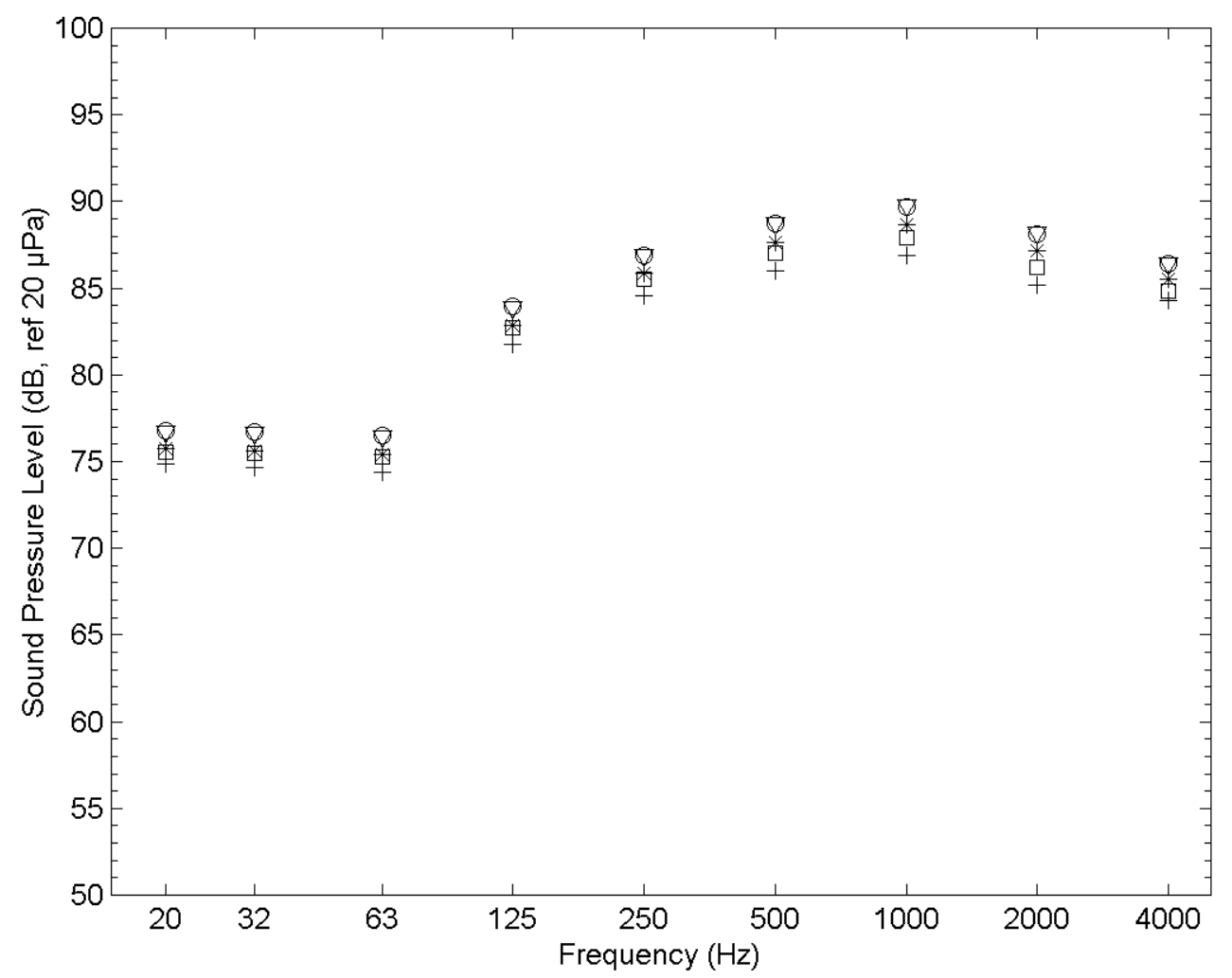

Figure B.11: Response at window location of 36 meters (window north of lane 7), all semi-truck sources active. $\nabla$ level of existing toll plaza; ${ }^{*}$ level with absorbing canopy; O level with absorbing barriers; level with absorbing barriers; + level with all modifications. 


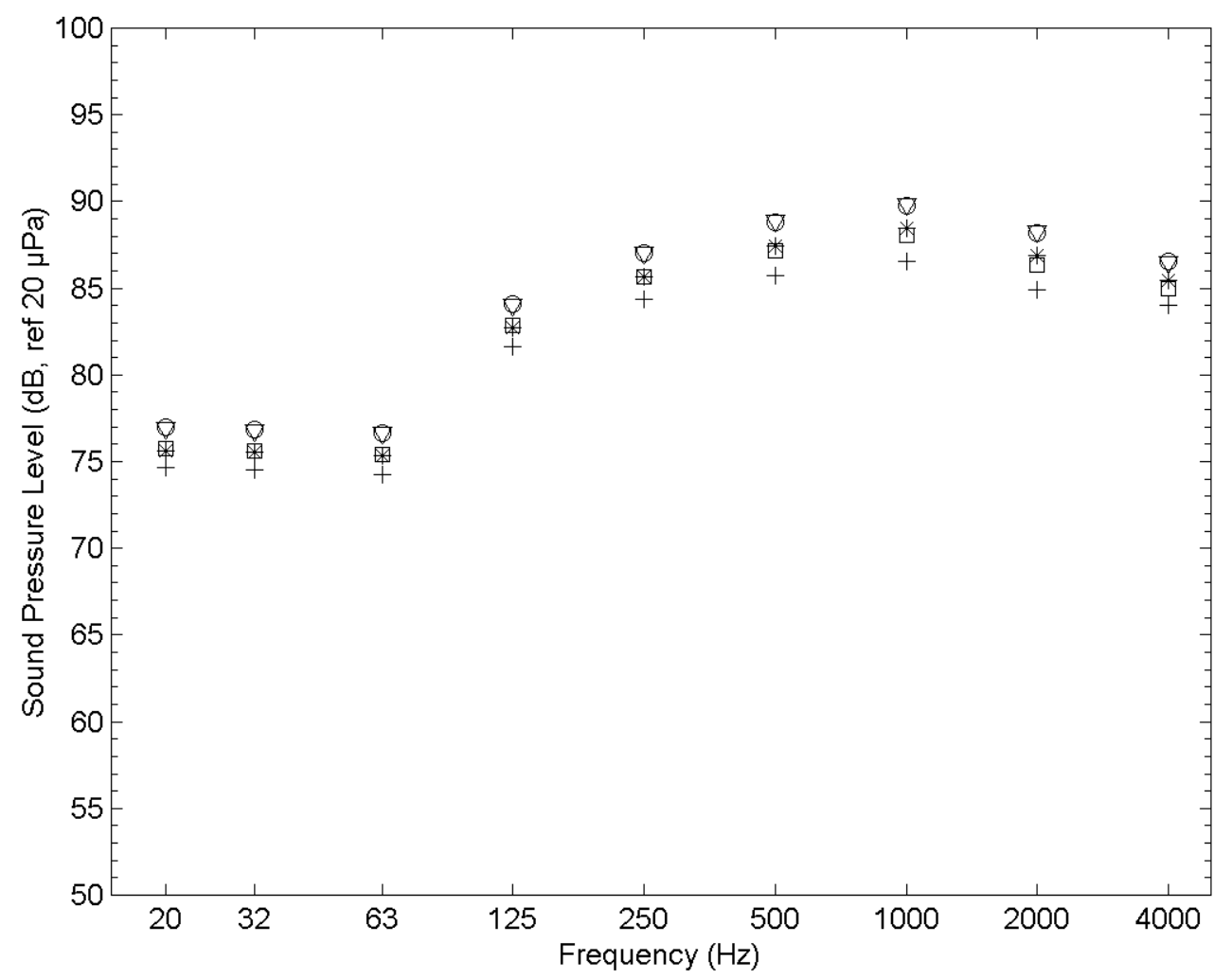

Figure B.12: Response at window location of 30 meters (window north of lane 8), all semi-truck sources active. $\nabla$ level of existing toll plaza; ${ }^{*}$ level with absorbing canopy; O level with absorbing barriers; level with absorbing barriers; + level with all modifications. 


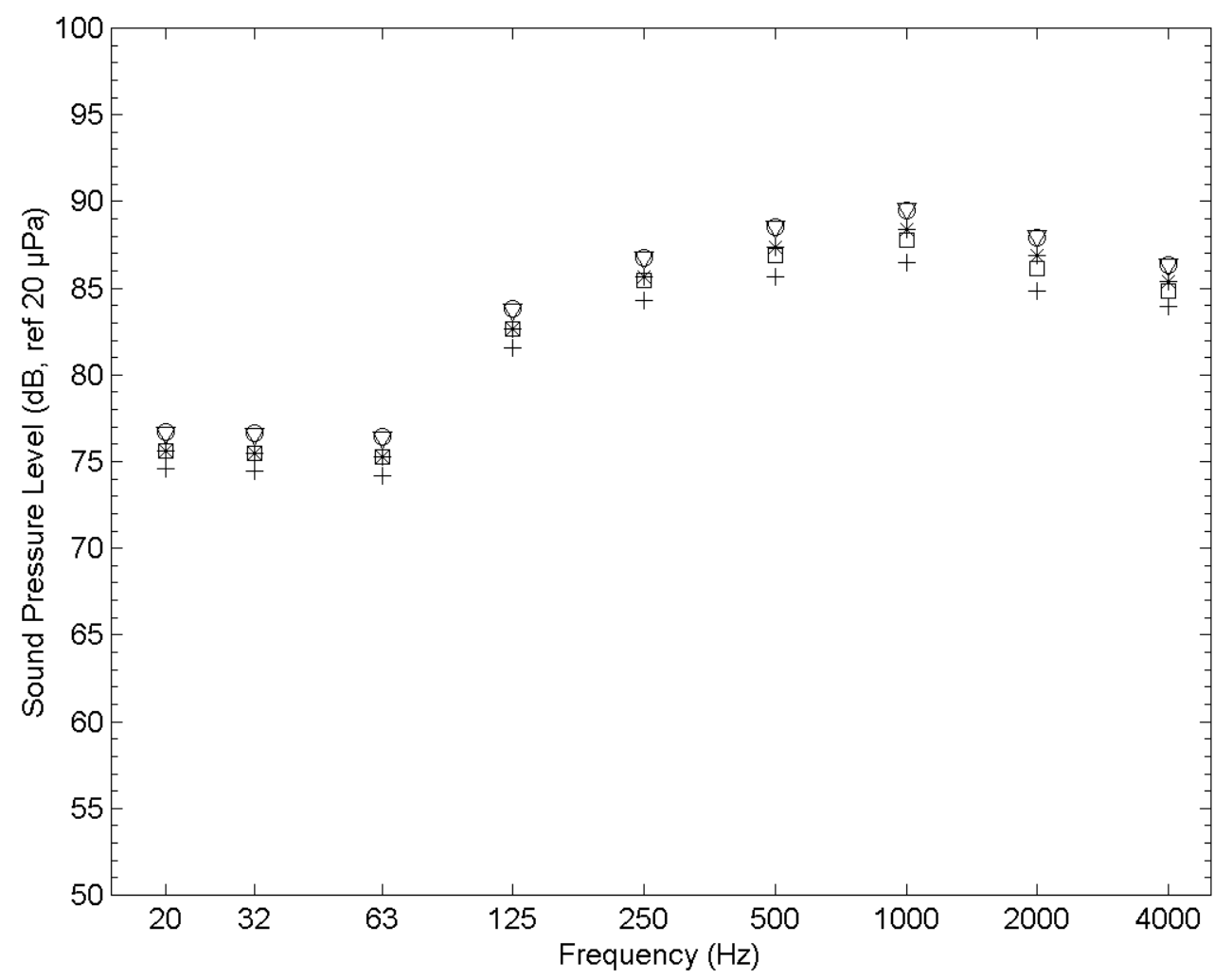

Figure B.13: Response at window location of 23 meters (window north of lane 9), all semi-truck sources active. $\nabla$ level of existing toll plaza; ${ }^{*}$ level with absorbing canopy; O level with absorbing barriers; level with absorbing barriers; + level with all modifications. 


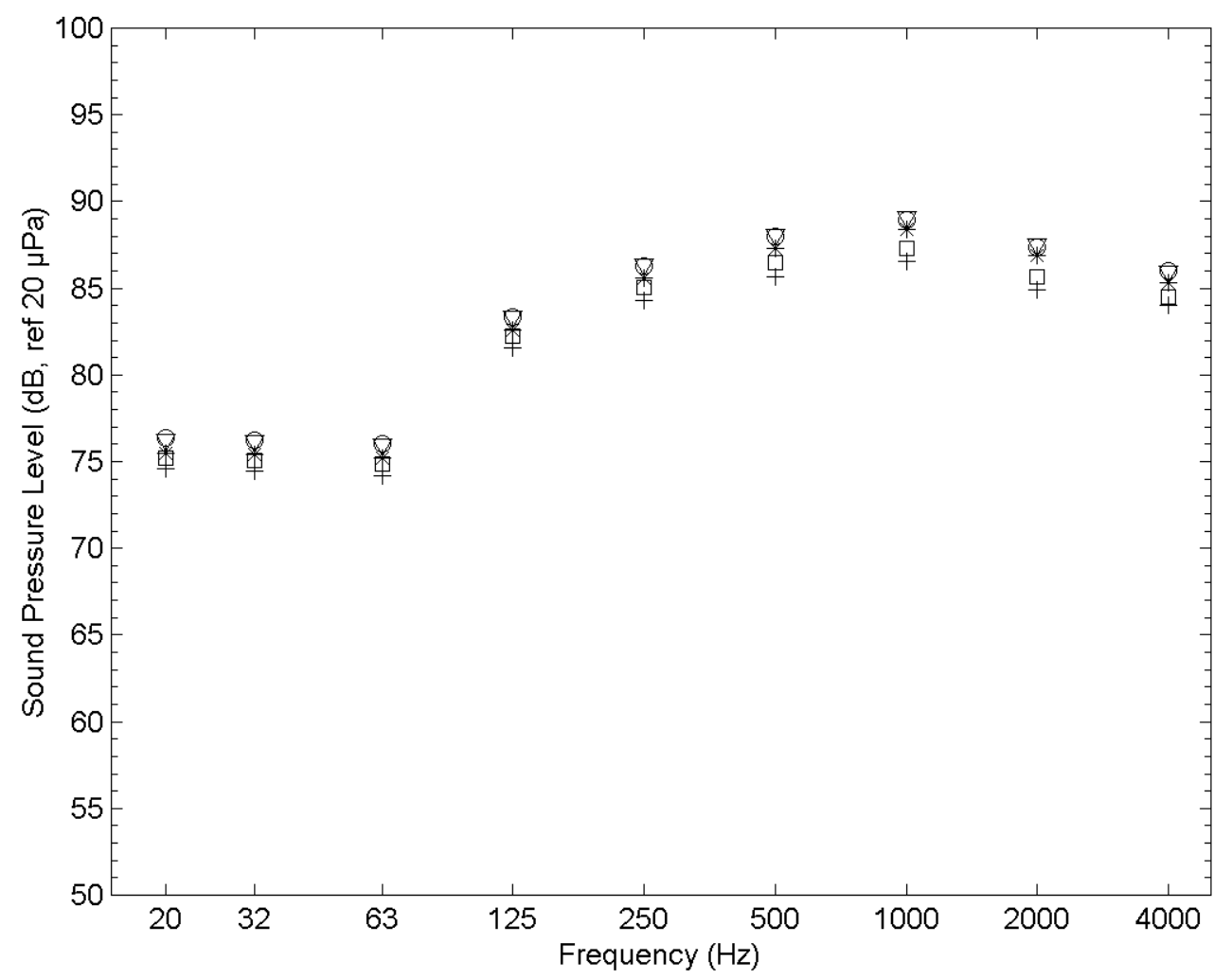

Figure B.14: Response at window location of 17 meters (window north of lane 10), all semi-truck sources active. $\nabla$ level of existing toll plaza; $*$ level with absorbing canopy; O level with absorbing barriers; level with absorbing barriers; + level with all modifications. 


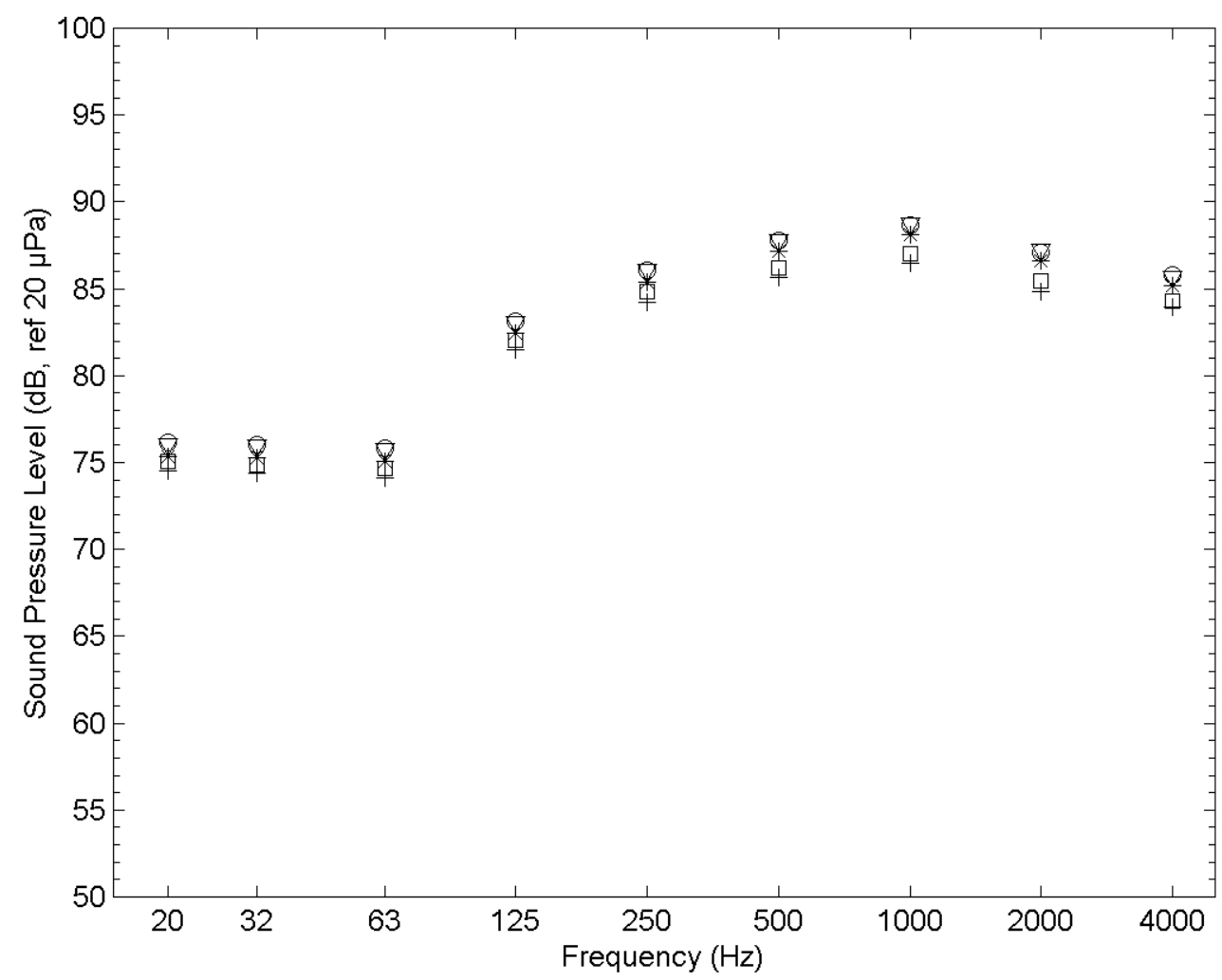

Figure B.15: Response at window location of 11 meters (window north of lane 11), all semi-truck sources active. $\nabla$ level of existing toll plaza; ${ }^{*}$ level with absorbing canopy; O level with absorbing barriers; level with absorbing barriers; + level with all modifications. 


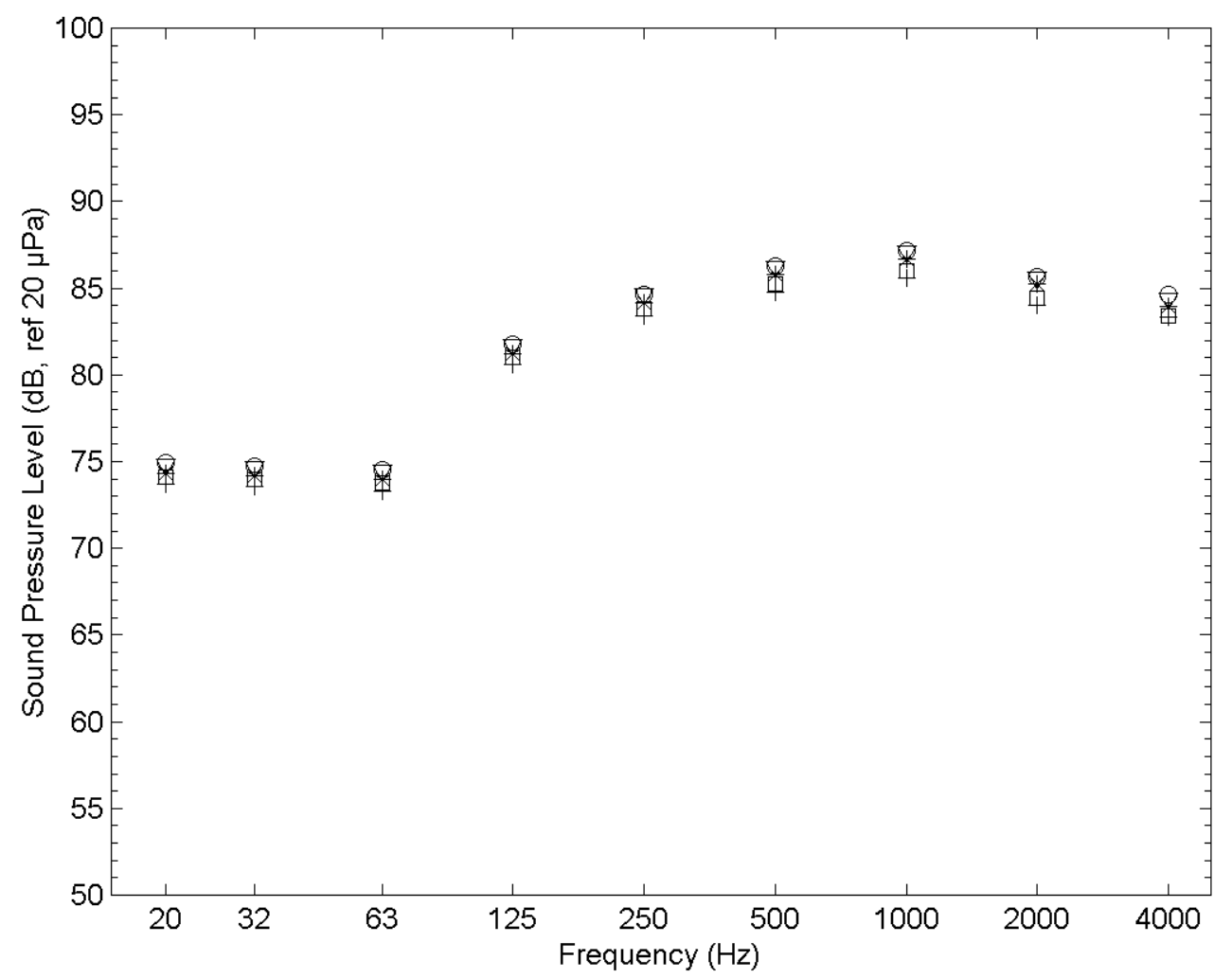

Figure B.16: Response at window location of 5 meters (window north of lane 12), all semi-truck sources active. $\nabla$ level of existing toll plaza; * level with absorbing canopy; O level with absorbing barriers; level with absorbing barriers; + level with all modifications. 


\section{B.3 Overall Level per Source Pair}

The figure in this section display the of the overall level of toll plaza models for a single source pair activated at a time. Sources represented semi-truck vehicles. Each figure shows the response at all window locations for a single source pair. 


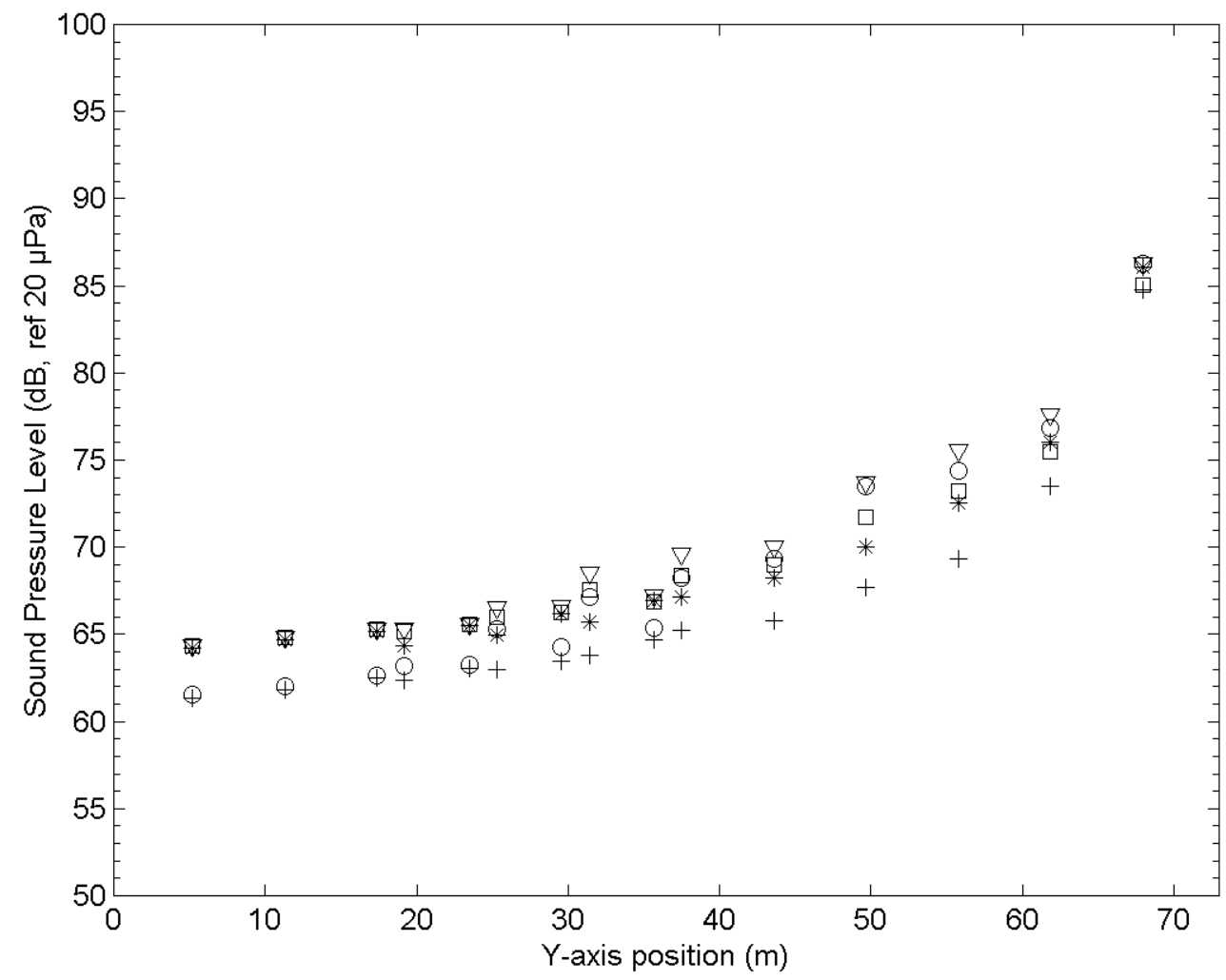

Figure B.17: Overall sound pressure level for sources 1 and 2 as a function of window location. $\nabla$ level of existing toll plaza; * level with absorbing canopy; $\mathrm{O}$ level with absorbing barriers; level with absorbing barriers; + level is all modifications. 


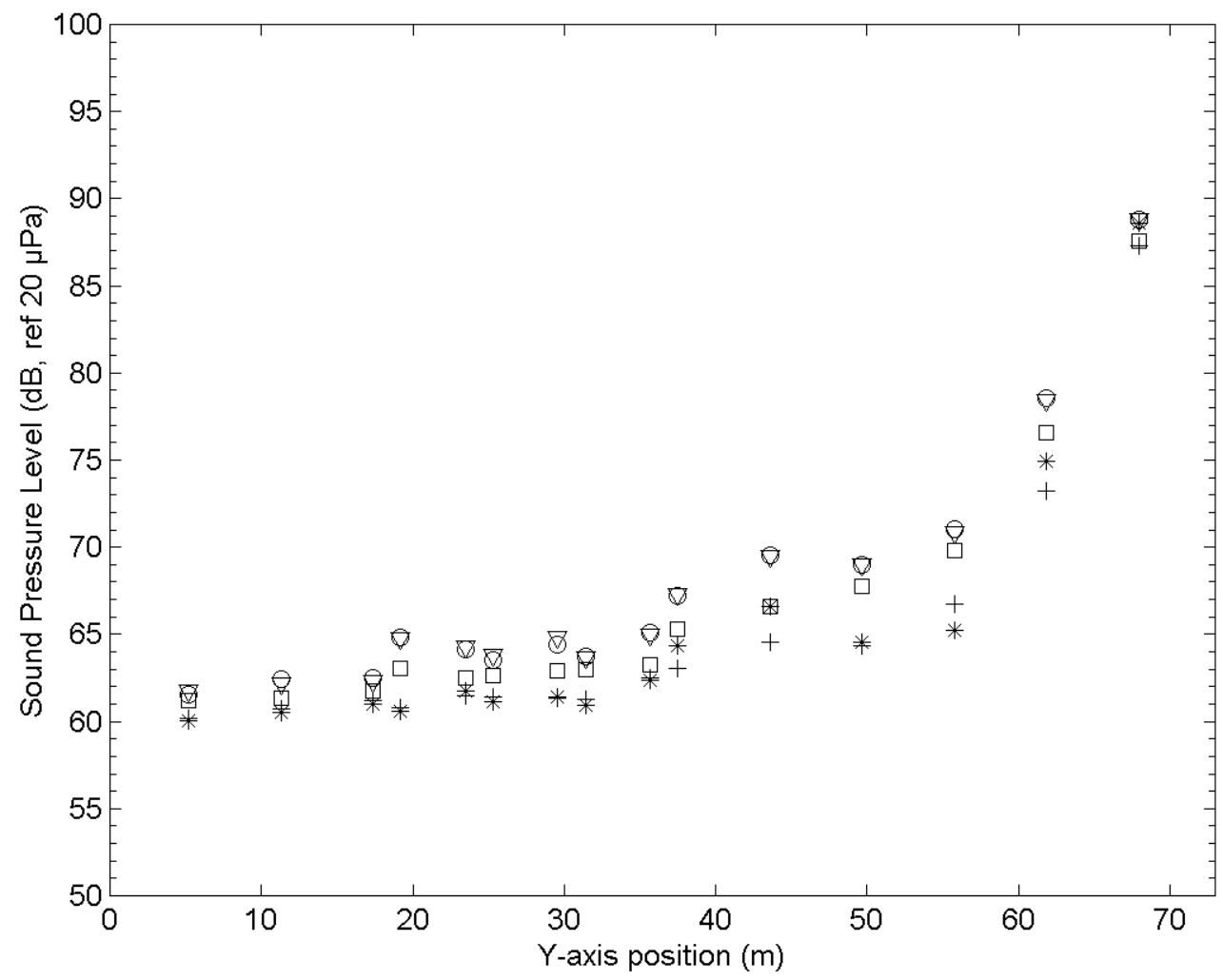

Figure B.18: Overall sound pressure level for sources 3 and 4 as a function of window location. $\nabla$ level of existing toll plaza; * level with absorbing canopy; $\mathrm{O}$ level with absorbing barriers; level with absorbing barriers; + level is all modifications. 


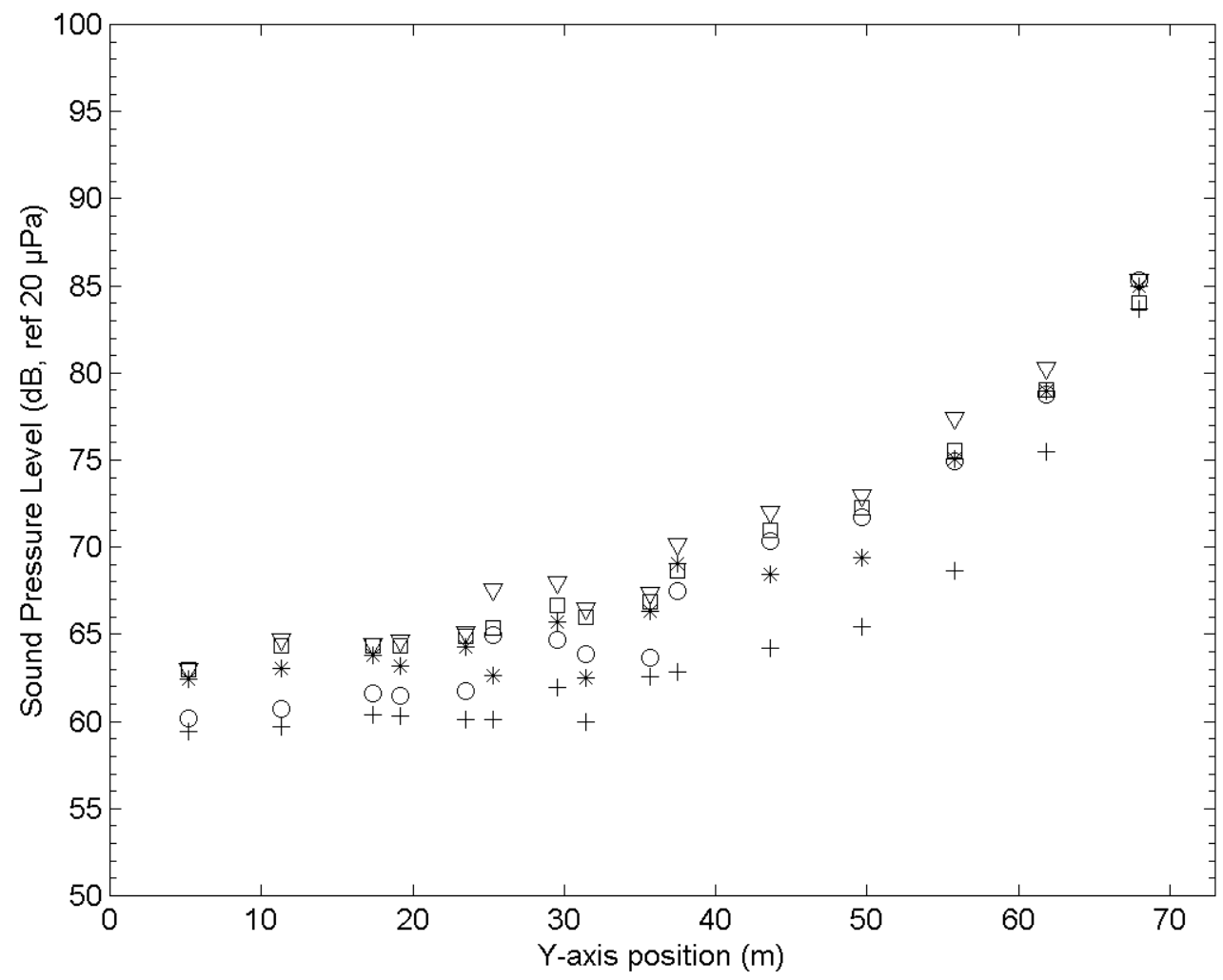

Figure B.19: Overall sound pressure level for sources 5 and 6 as a function of window location. $\nabla$ level of existing toll plaza; * level with absorbing canopy; $\mathrm{O}$ level with absorbing barriers; level with absorbing barriers; + level is all modifications. 


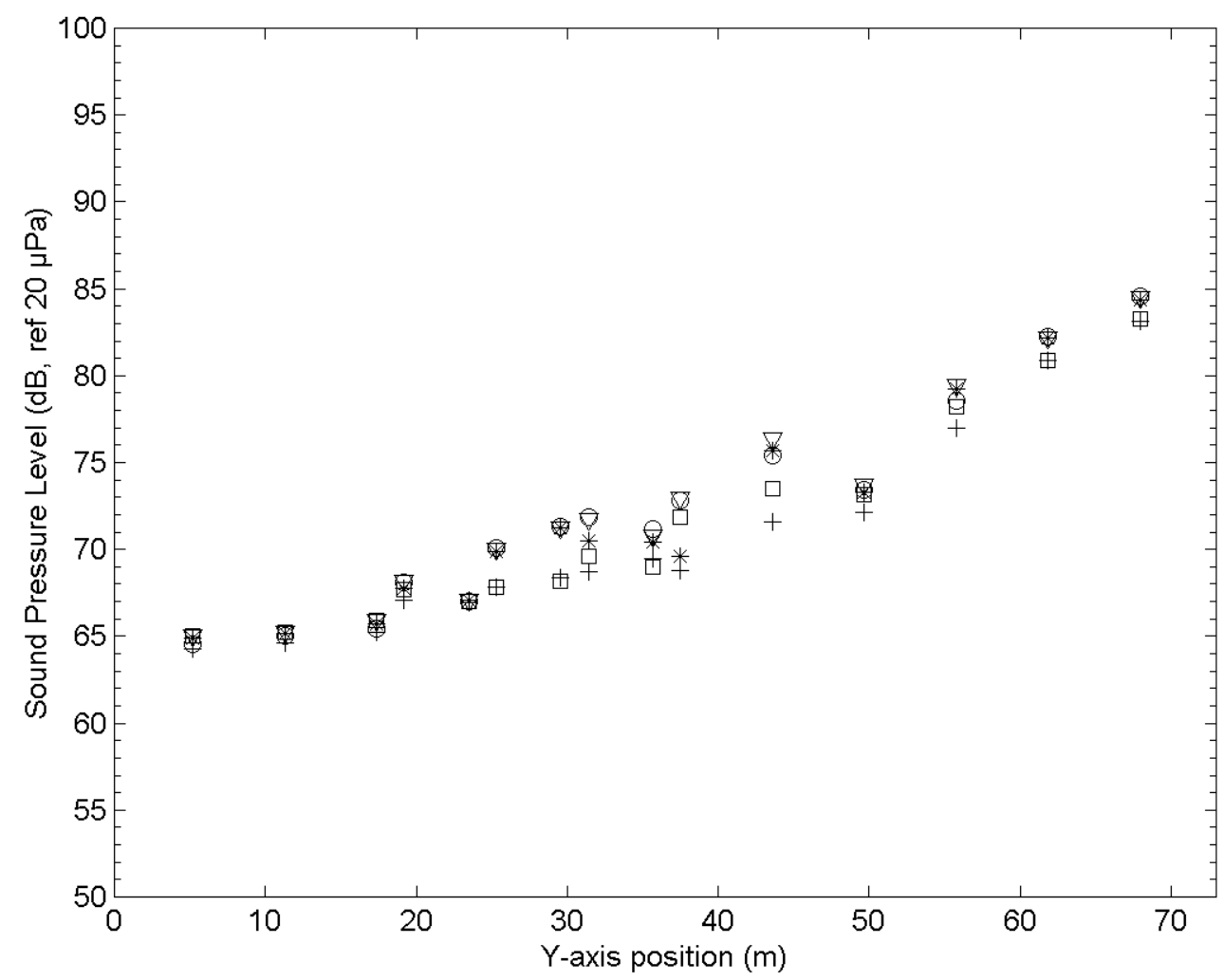

Figure B.20: Overall sound pressure level for sources 7 and 8 as a function of window location. $\nabla$ level of existing toll plaza; * level with absorbing canopy; $\mathrm{O}$ level with absorbing barriers; level with absorbing barriers; + level is all modifications. 


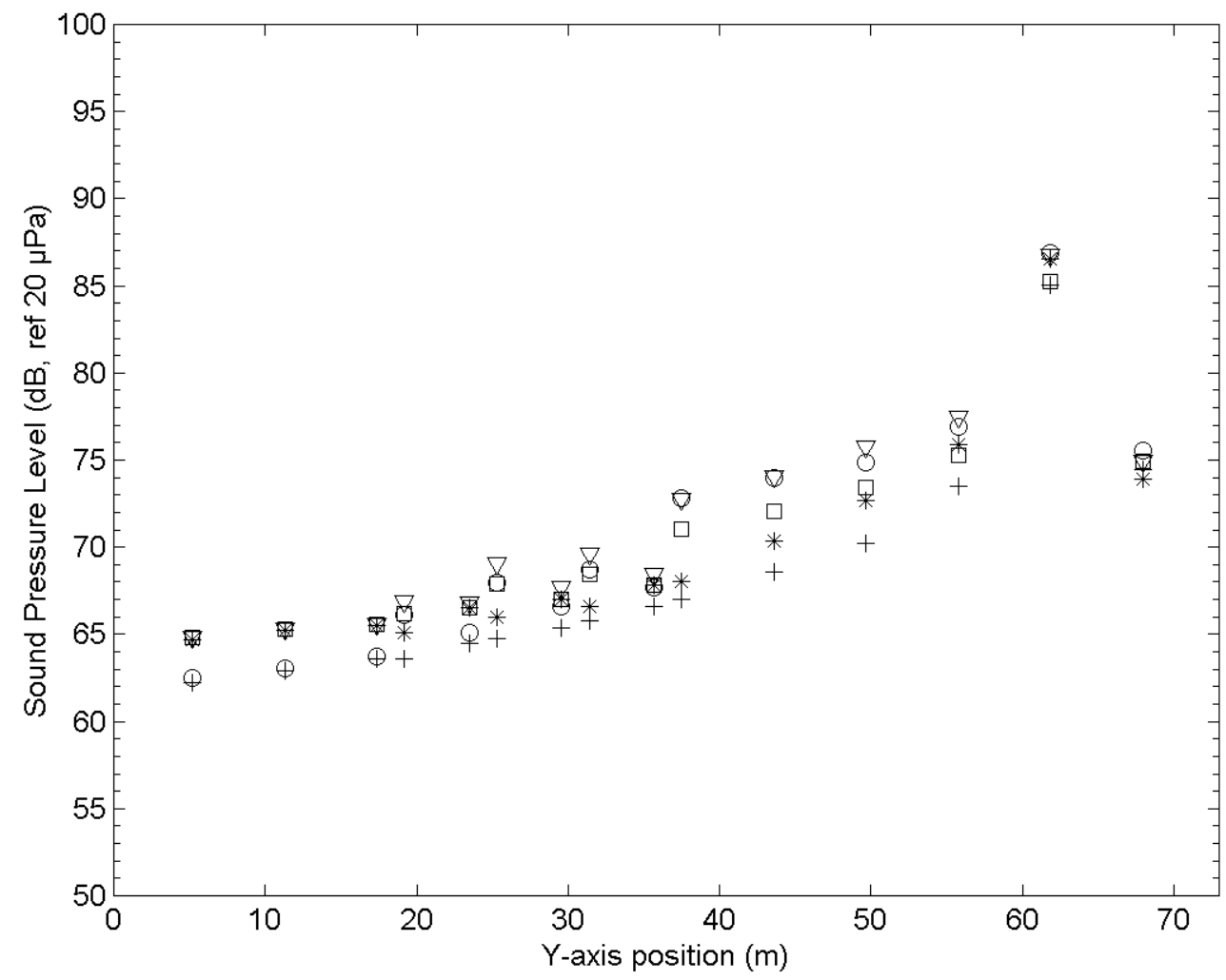

Figure B.21: Overall sound pressure level for sources 9 and 10 as a function of window location. $\nabla$ level of existing toll plaza; * level with absorbing canopy; $\mathrm{O}$ level with absorbing barriers; level with absorbing barriers; + level is all modifications. 


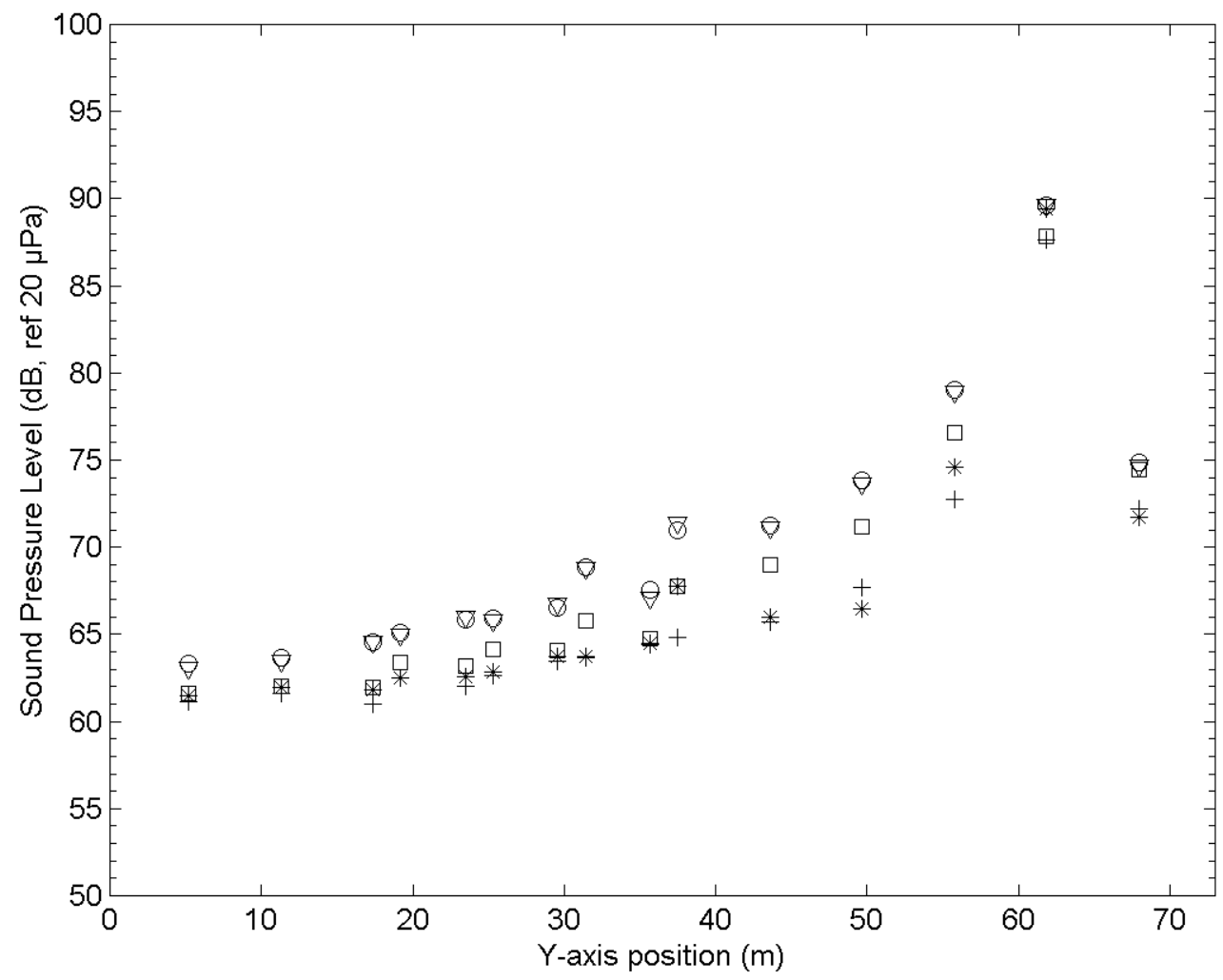

Figure B.22: Overall sound pressure level for sources 11 and 12 as a function of window location. $\nabla$ level of existing toll plaza; * level with absorbing canopy; $\mathrm{O}$ level with absorbing barriers; level with absorbing barriers; + level is all modifications. 


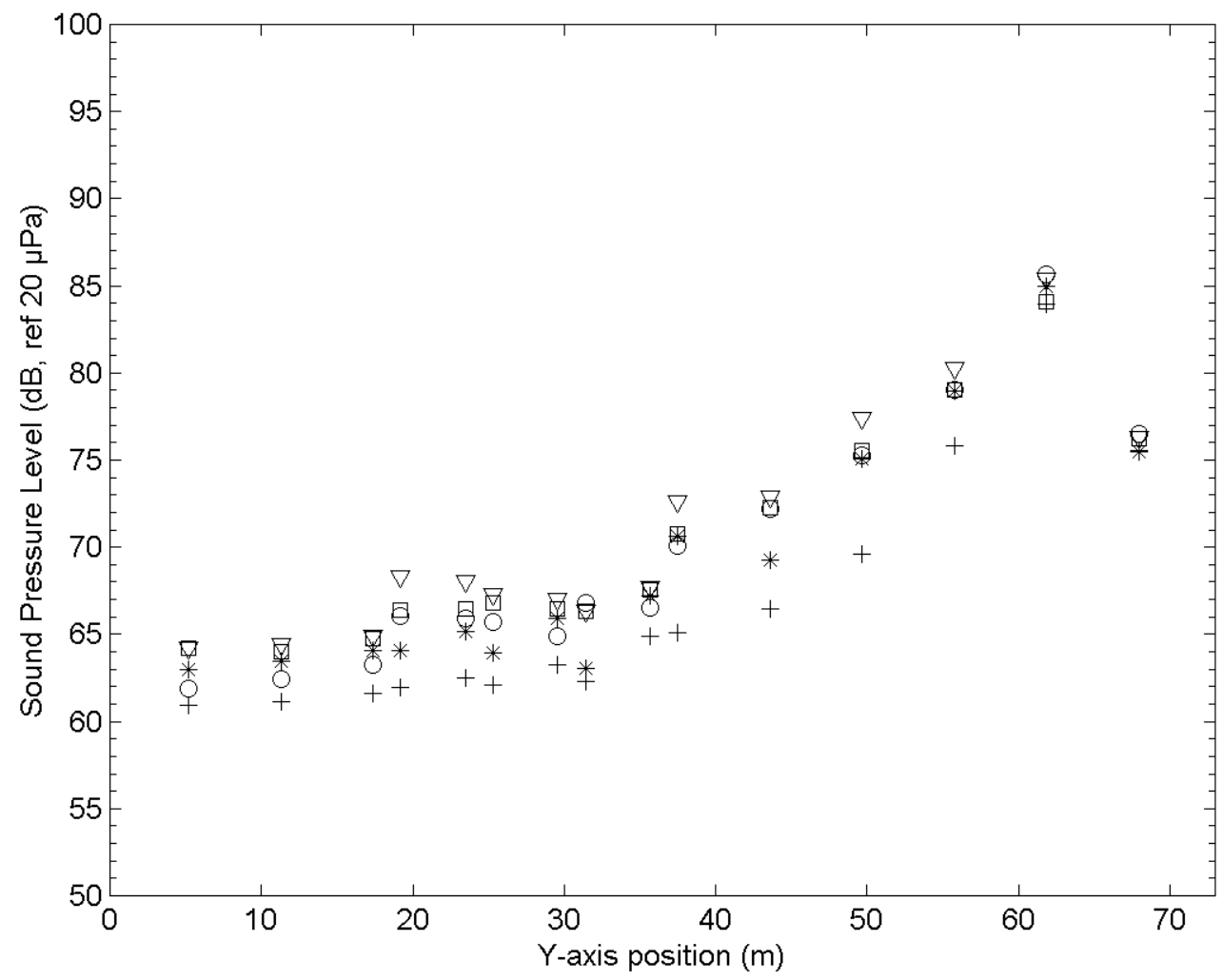

Figure B.23: Overall sound pressure level for sources 13 and 14 as a function of window location. $\nabla$ level of existing toll plaza; * level with absorbing canopy; O level with absorbing barriers; level with absorbing barriers; + level is all modifications. 


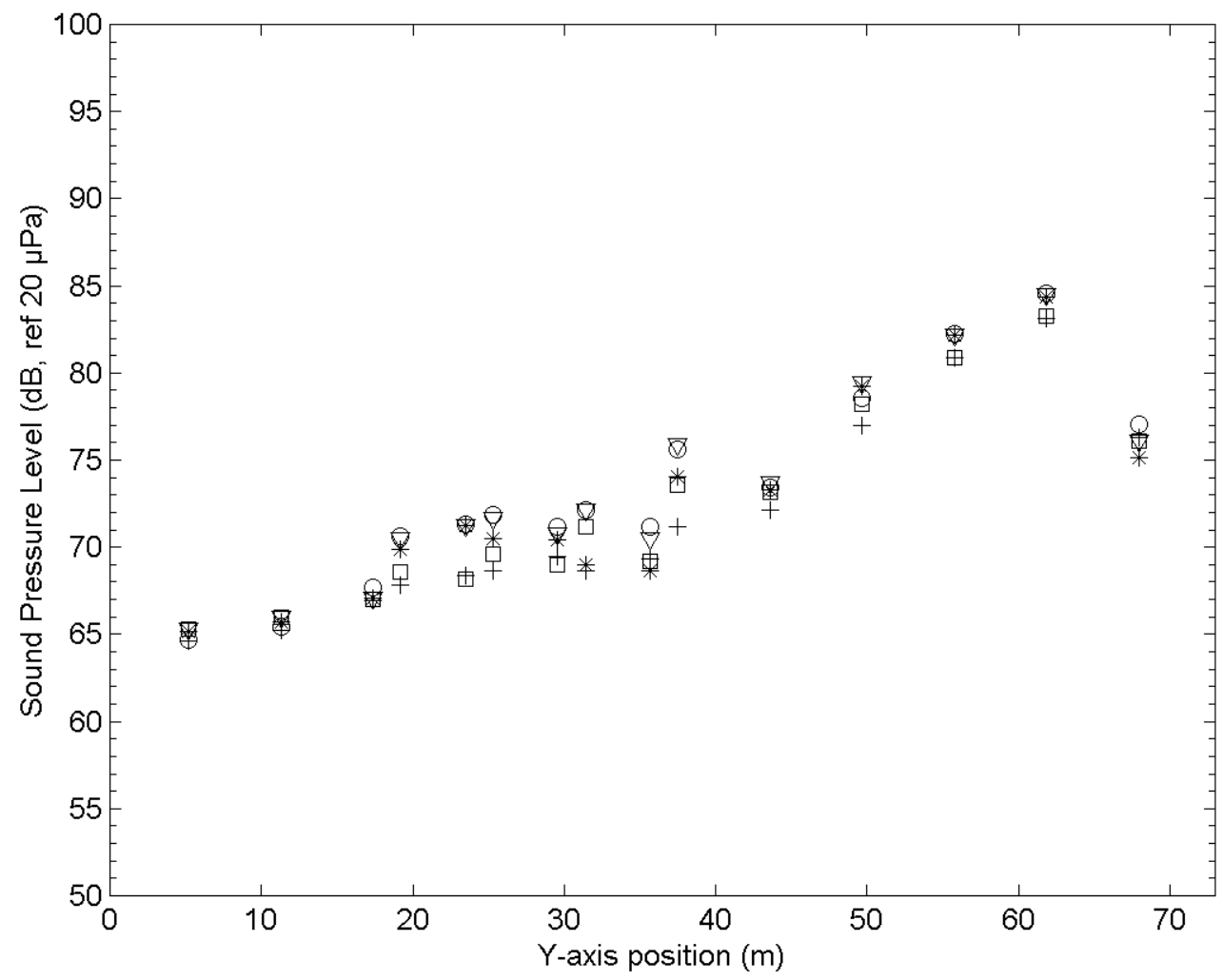

Figure B.24: Overall sound pressure level for sources 15 and 16 as a function of window location. $\nabla$ level of existing toll plaza; * level with absorbing canopy; $\mathrm{O}$ level with absorbing barriers; level with absorbing barriers; + level is all modifications. 


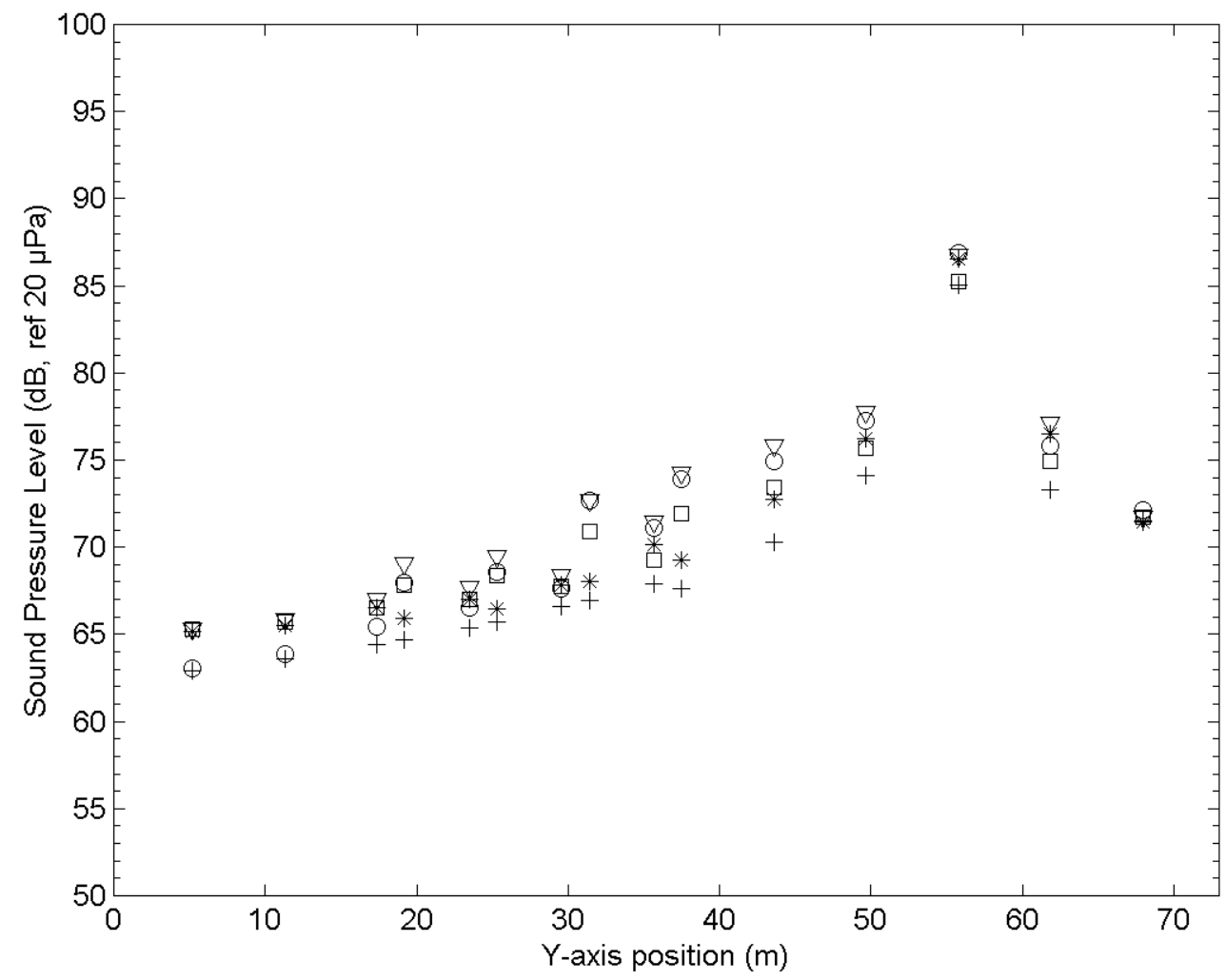

Figure B.25: Overall sound pressure level for sources 17 and 18 as a function of window location. $\nabla$ level of existing toll plaza; * level with absorbing canopy; $\mathrm{O}$ level with absorbing barriers; level with absorbing barriers; + level is all modifications. 


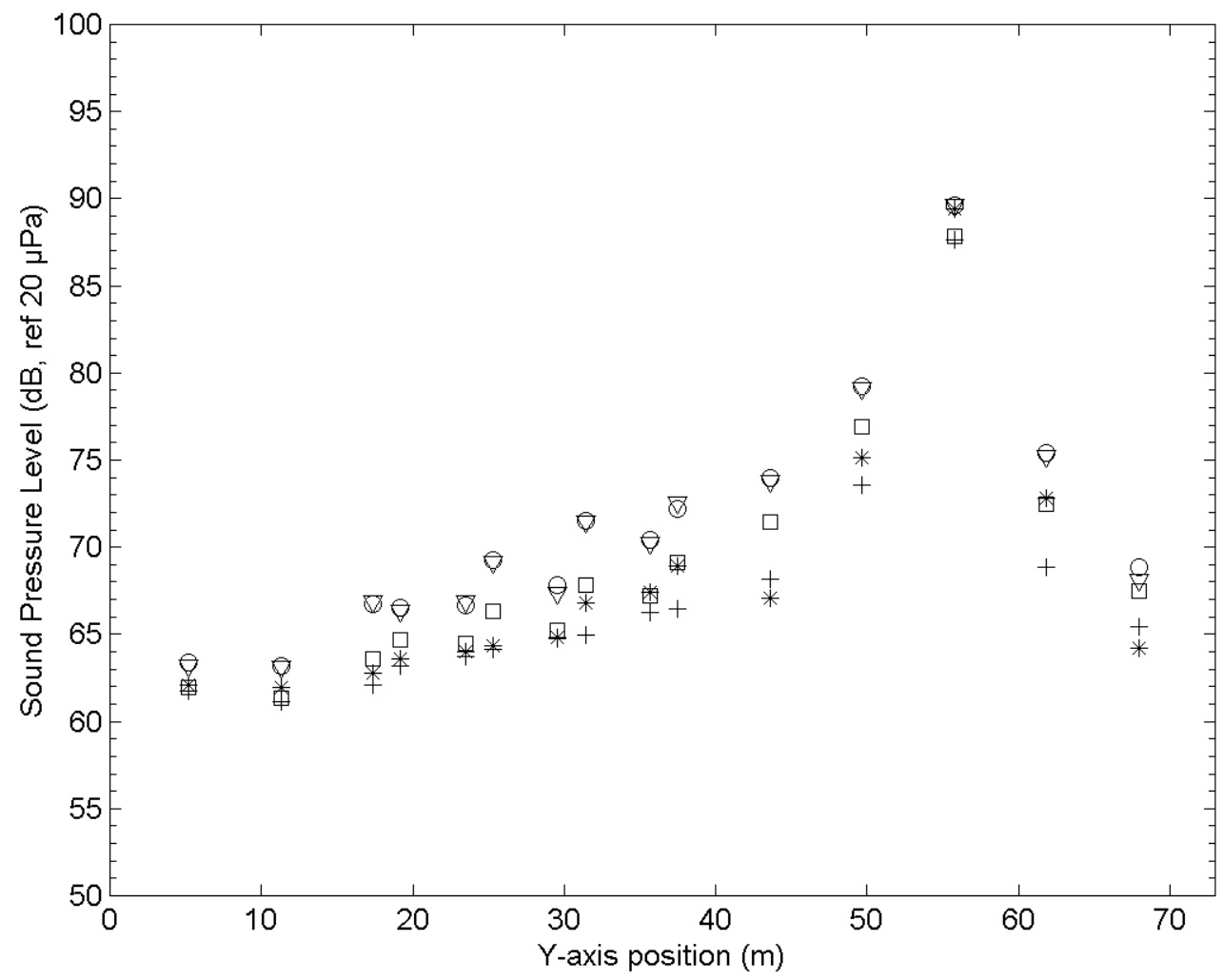

Figure B.26: Overall sound pressure level for sources 19 and 20 as a function of window location. $\nabla$ level of existing toll plaza; * level with absorbing canopy; O level with absorbing barriers; level with absorbing barriers; + level is all modifications. 


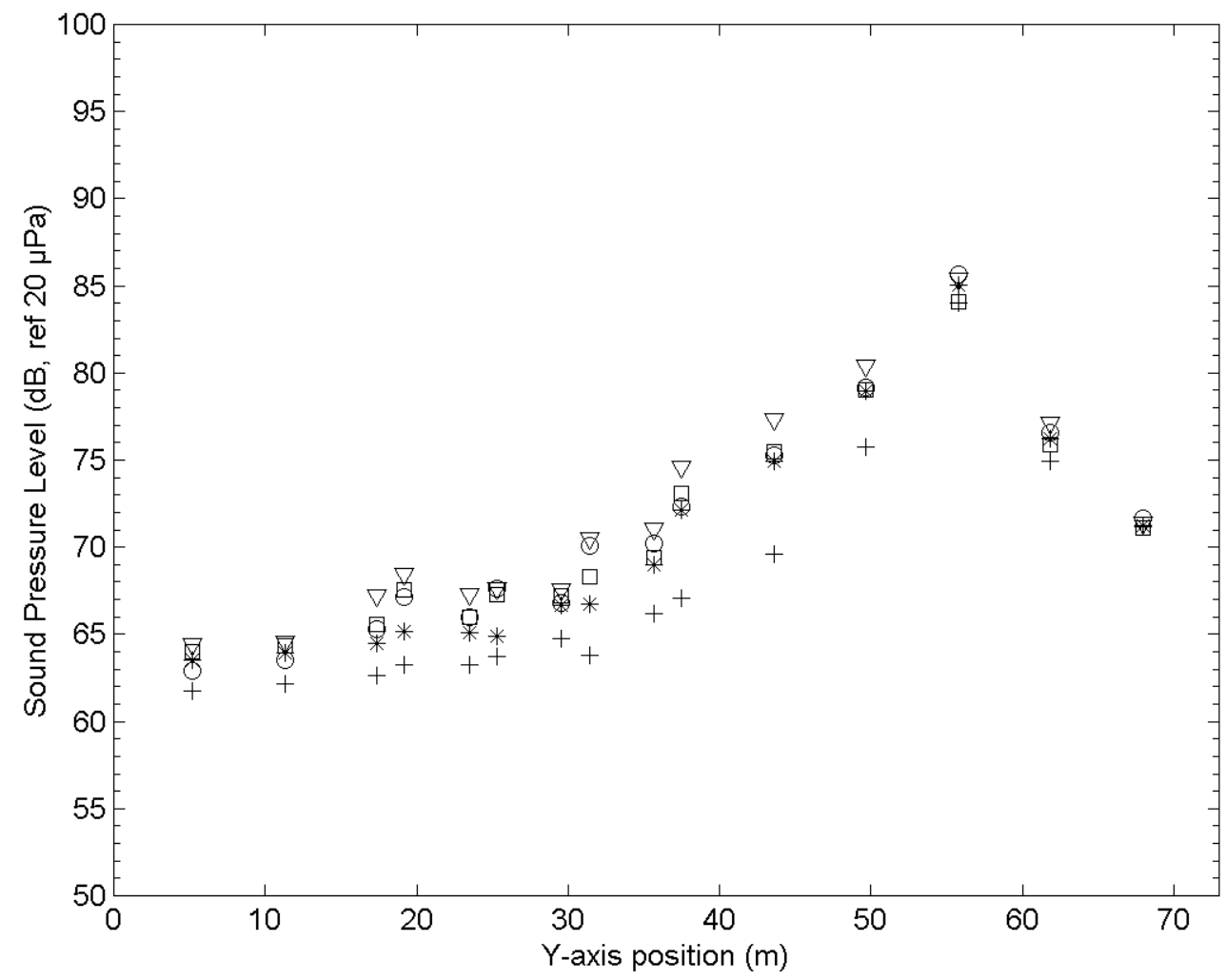

Figure B.27: Overall sound pressure level for sources 21 and 22 as a function of window location. $\nabla$ level of existing toll plaza; * level with absorbing canopy; $\mathrm{O}$ level with absorbing barriers; level with absorbing barriers; + level is all modifications. 


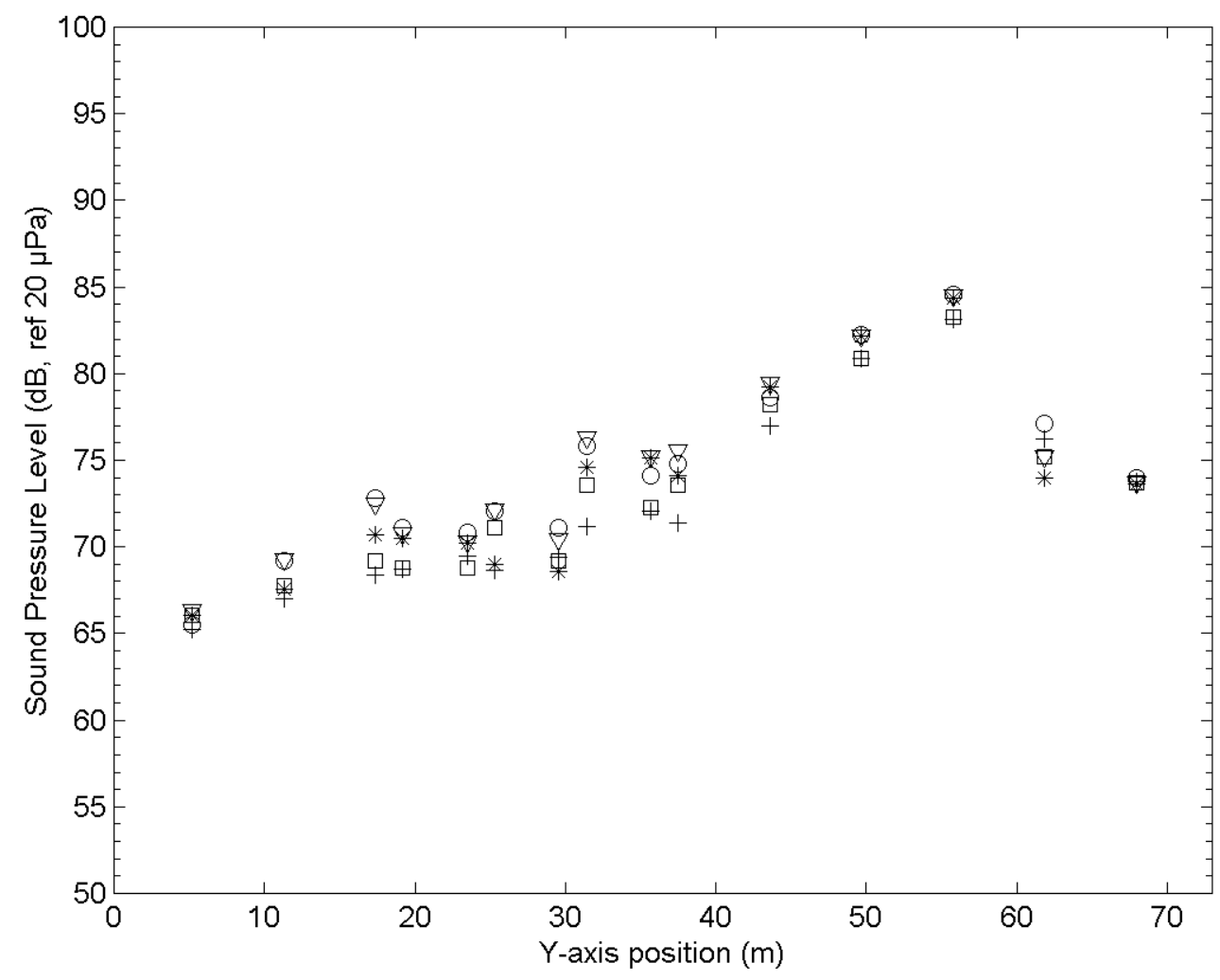

Figure B.28: Overall sound pressure level for sources 23 and 24 as a function of window location. $\nabla$ level of existing toll plaza; * level with absorbing canopy; $\mathrm{O}$ level with absorbing barriers; level with absorbing barriers; + level is all modifications. 


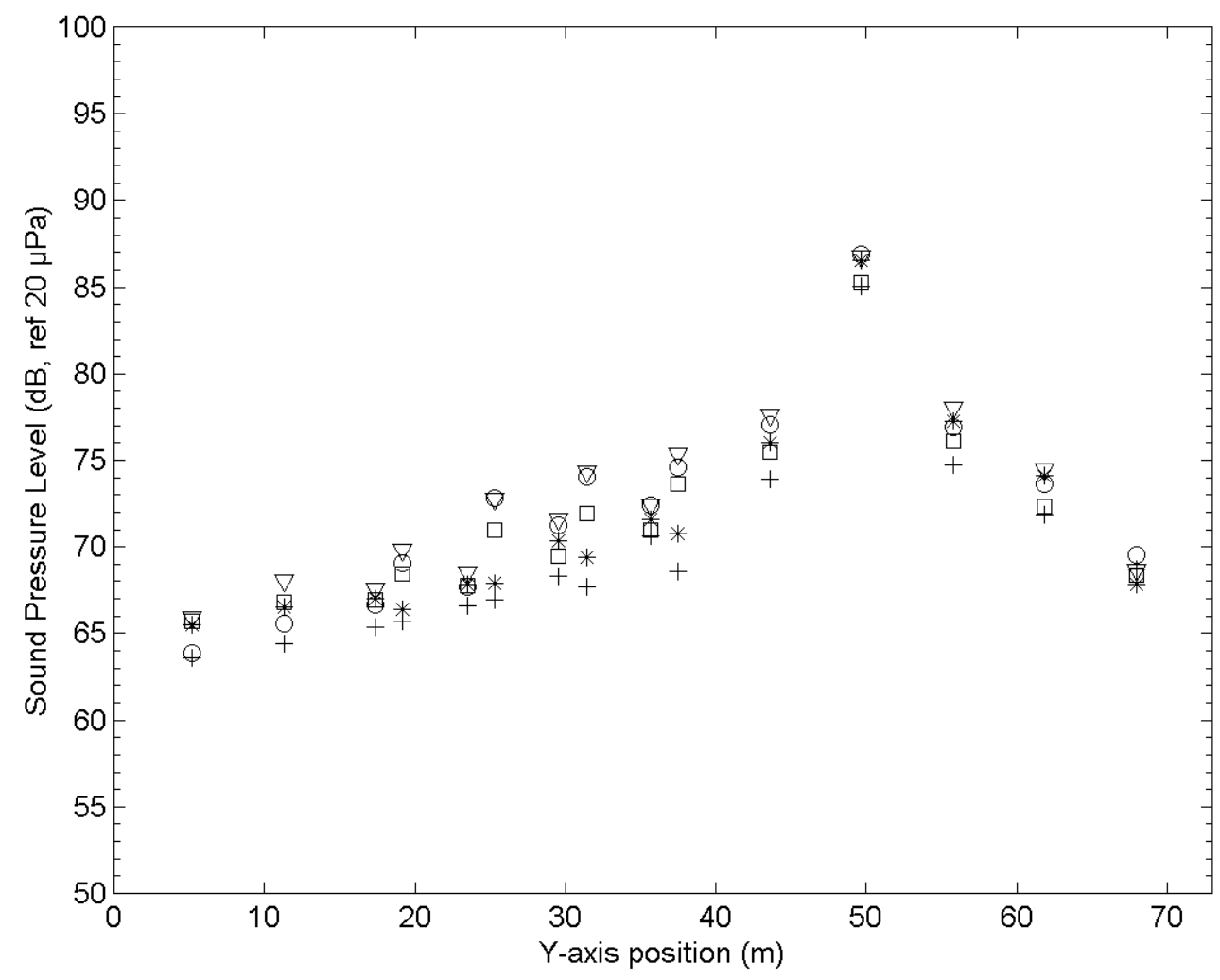

Figure B.29: Overall sound pressure level for sources 25 and 26 as a function of window location. $\nabla$ level of existing toll plaza; * level with absorbing canopy; O level with absorbing barriers; level with absorbing barriers; + level is all modifications. 


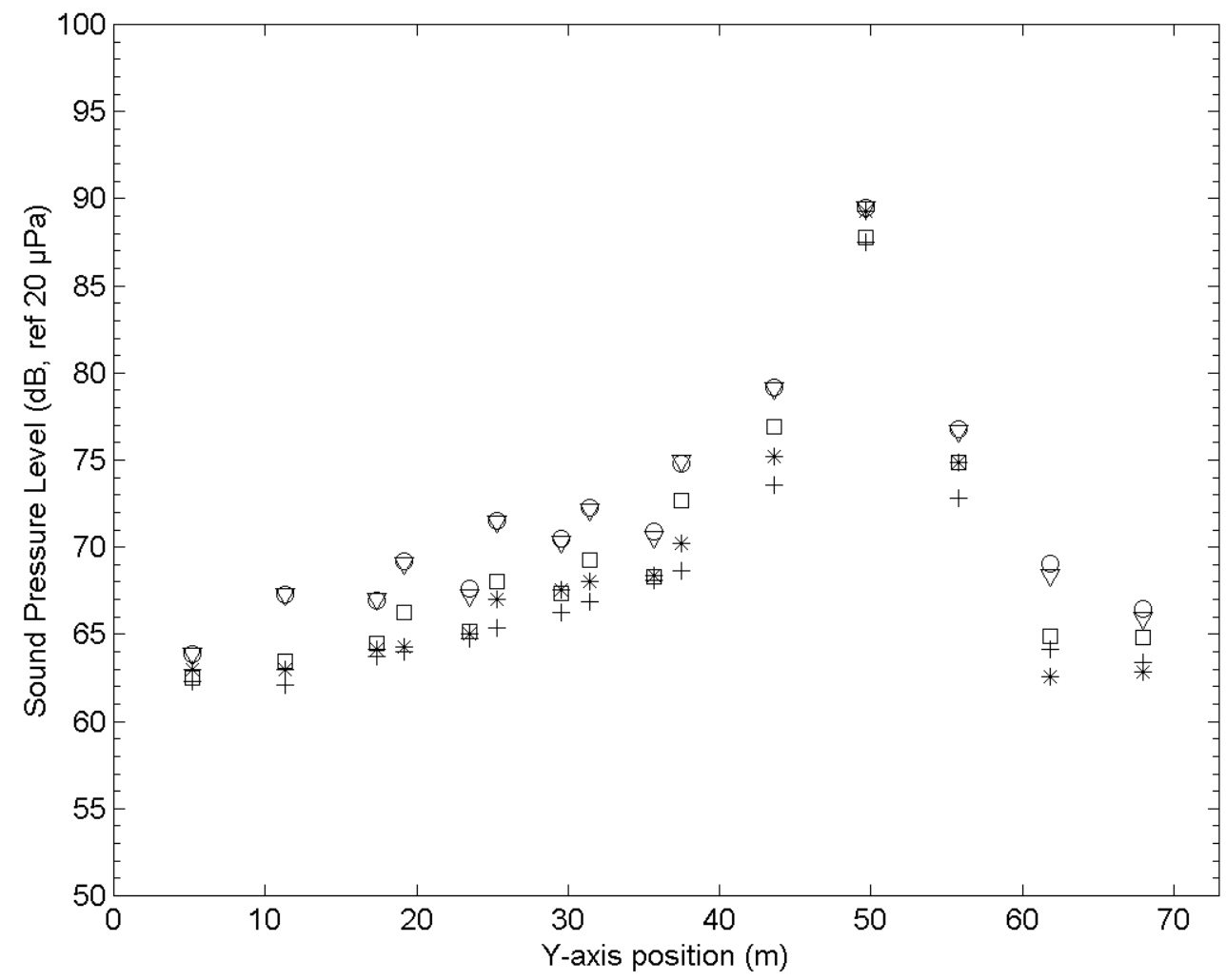

Figure B.30: Overall sound pressure level for sources 27 and 28 as a function of window location. $\nabla$ level of existing toll plaza; * level with absorbing canopy; $\mathrm{O}$ level with absorbing barriers; level with absorbing barriers; + level is all modifications. 


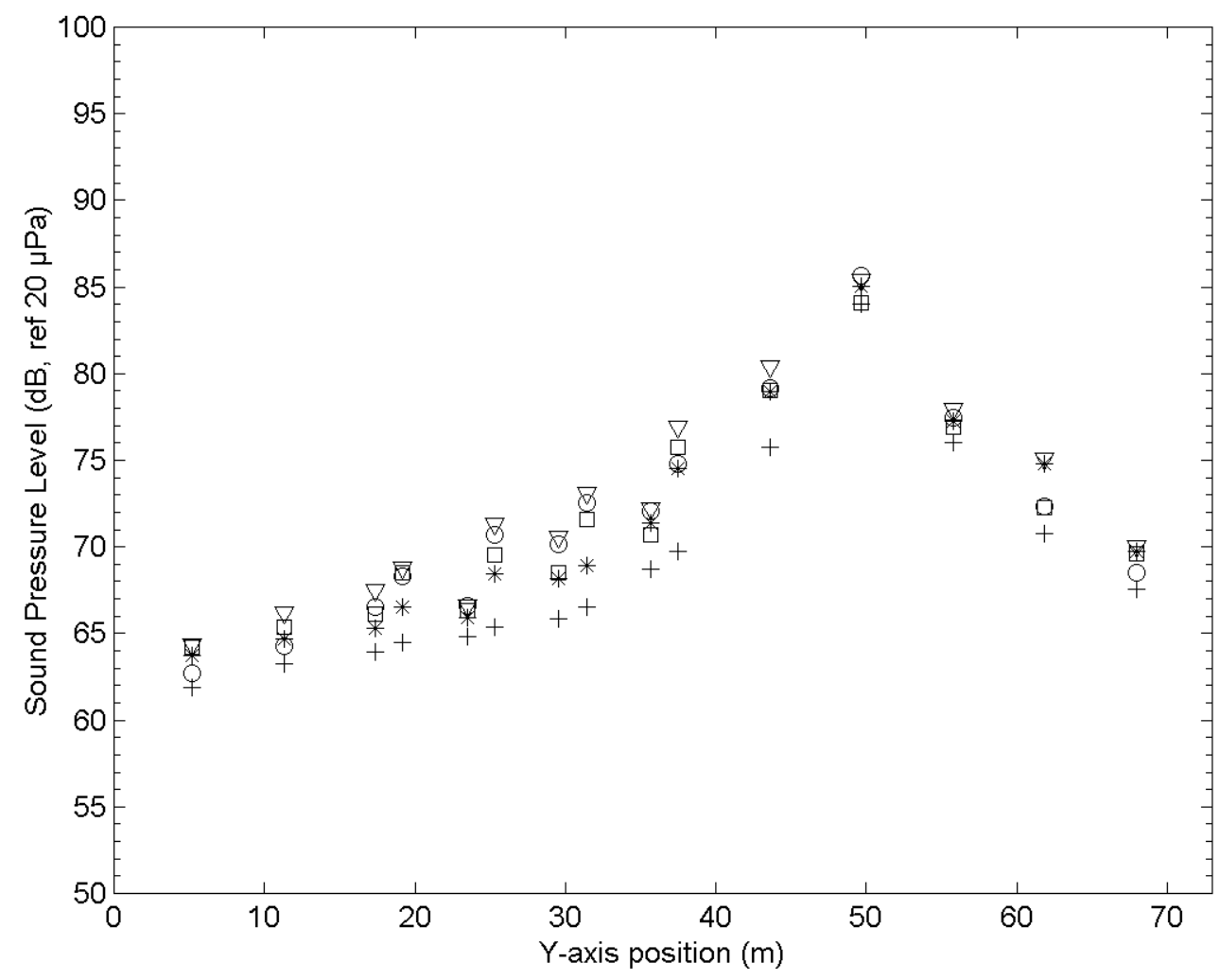

Figure B.31: Overall sound pressure level for sources 29 and 30 as a function of window location. $\nabla$ level of existing toll plaza; * level with absorbing canopy; $\mathrm{O}$ level with absorbing barriers; level with absorbing barriers; + level is all modifications. 


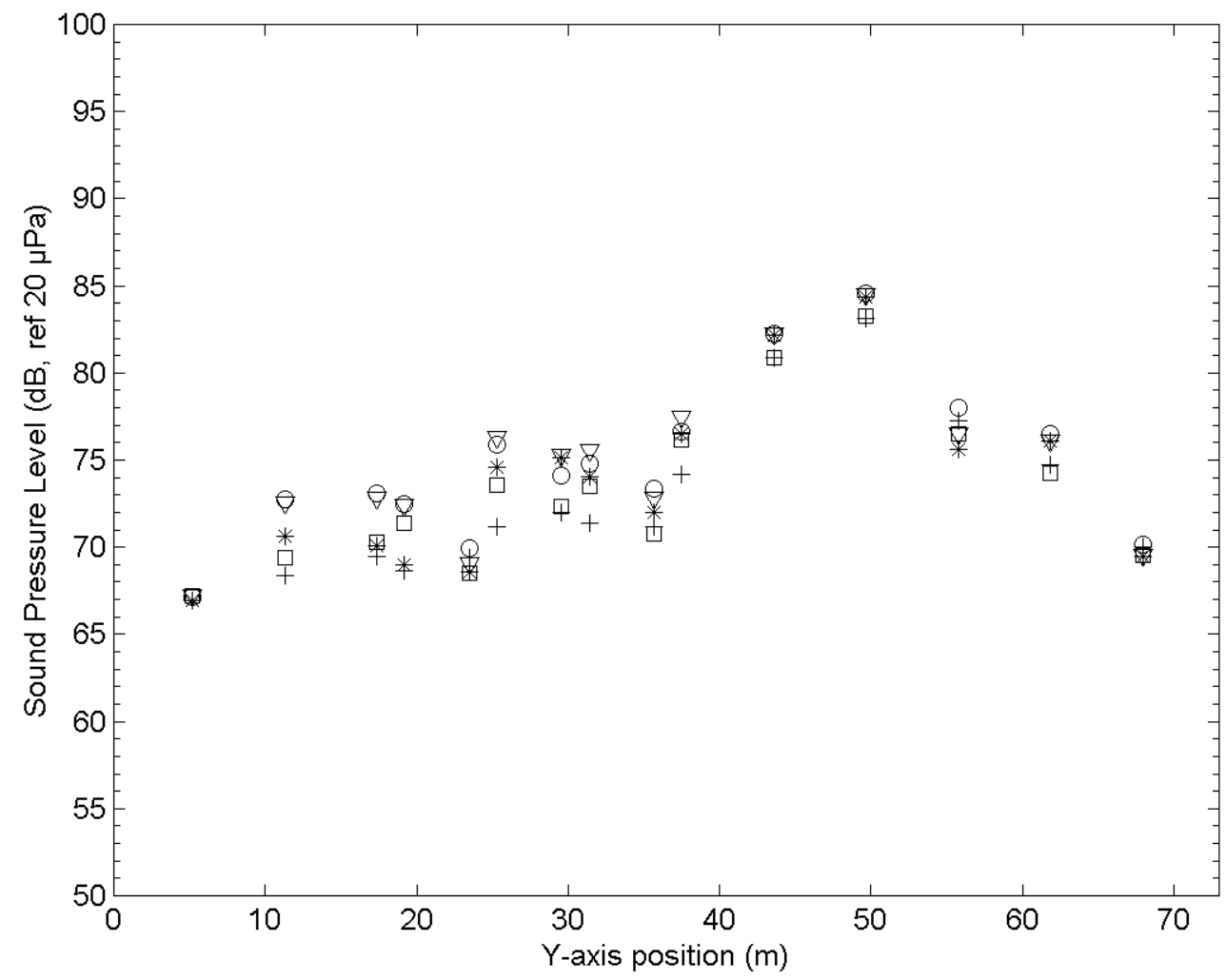

Figure B.32: Overall sound pressure level for sources 31 and 32 as a function of window location. $\nabla$ level of existing toll plaza; * level with absorbing canopy; $\mathrm{O}$ level with absorbing barriers; level with absorbing barriers; + level is all modifications. 


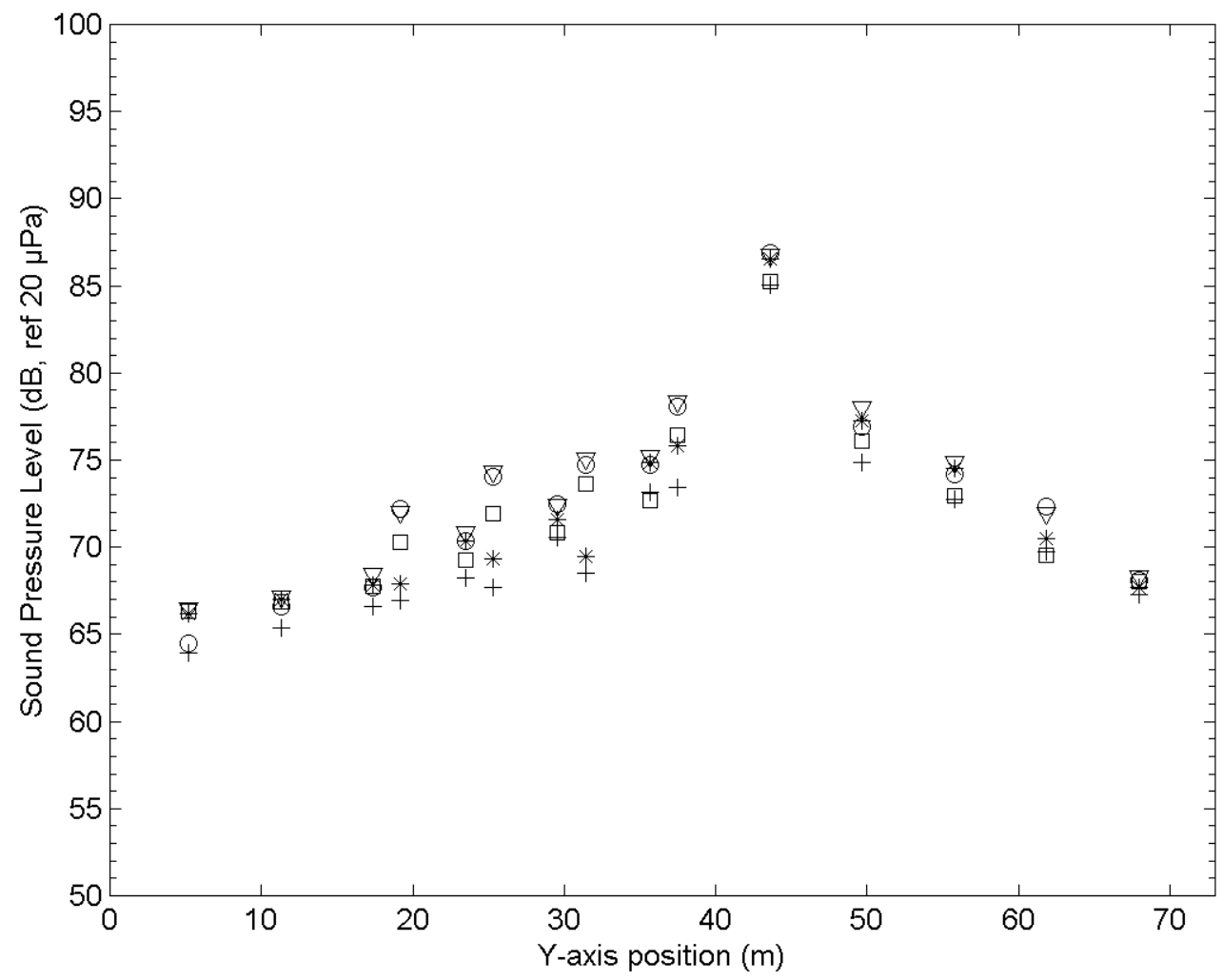

Figure B.33: Overall sound pressure level for sources 33 and 34 as a function of window location. $\nabla$ level of existing toll plaza; * level with absorbing canopy; O level with absorbing barriers; level with absorbing barriers; + level is all modifications. 


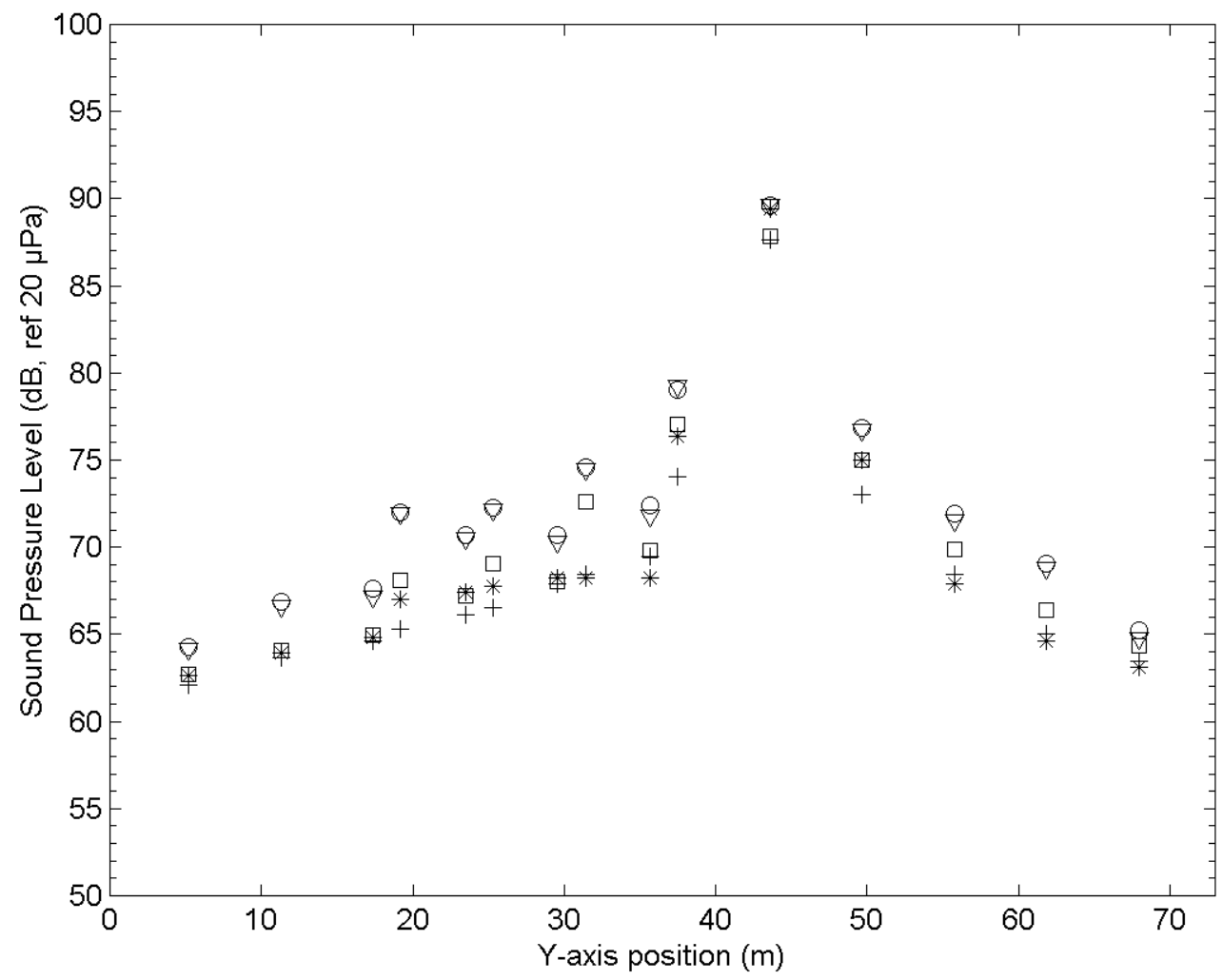

Figure B.34: Overall sound pressure level for sources 35 and 36 as a function of window location. $\nabla$ level of existing toll plaza; * level with absorbing canopy; $\mathrm{O}$ level with absorbing barriers; level with absorbing barriers; + level is all modifications. 


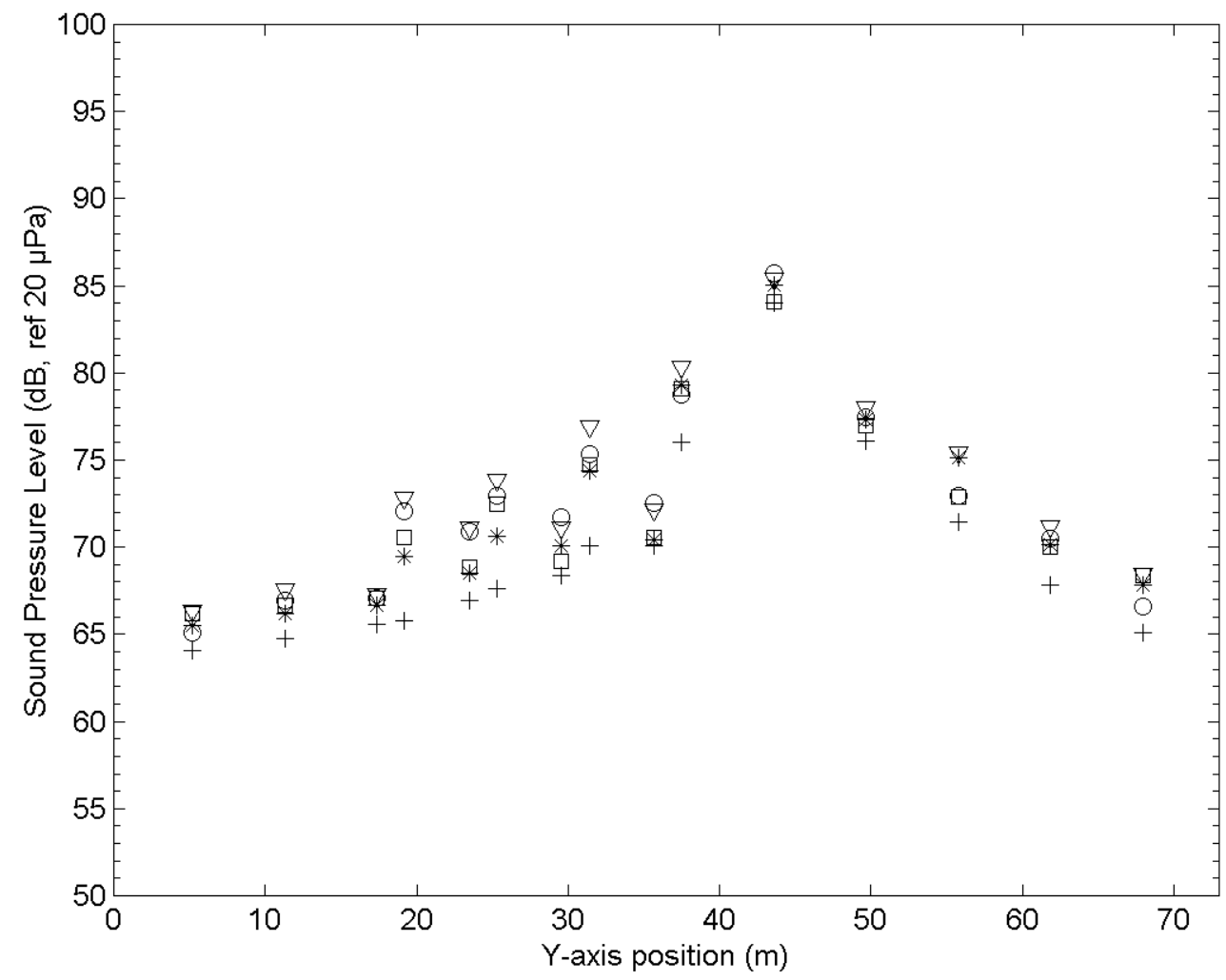

Figure B.35: Overall sound pressure level for sources 37 and 38 as a function of window location. $\nabla$ level of existing toll plaza; * level with absorbing canopy; $\mathrm{O}$ level with absorbing barriers; level with absorbing barriers; + level is all modifications. 


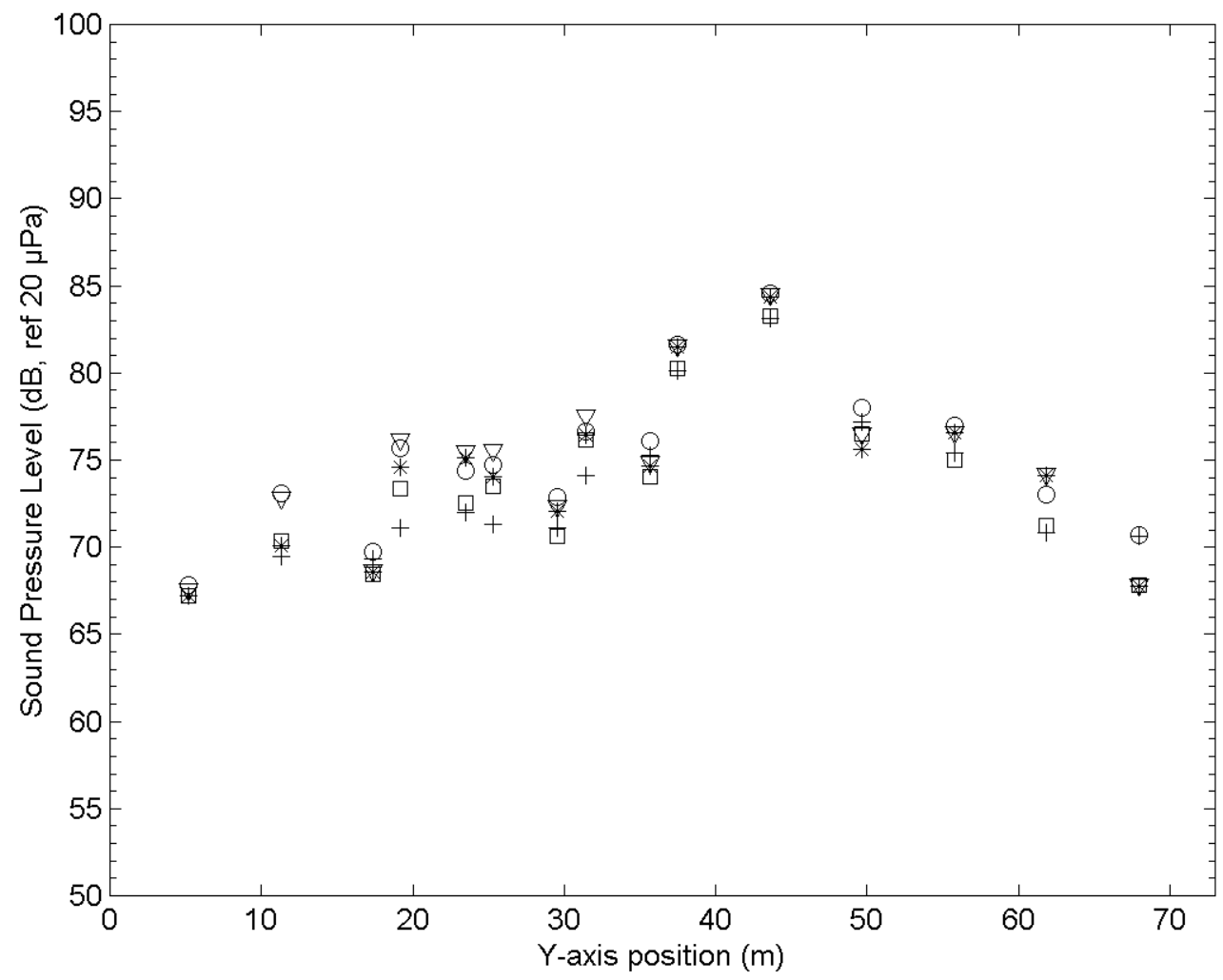

Figure B.36: Overall sound pressure level for sources 39 and 40 as a function of window location. $\nabla$ level of existing toll plaza; * level with absorbing canopy; $\mathrm{O}$ level with absorbing barriers; level with absorbing barriers; + level is all modifications. 


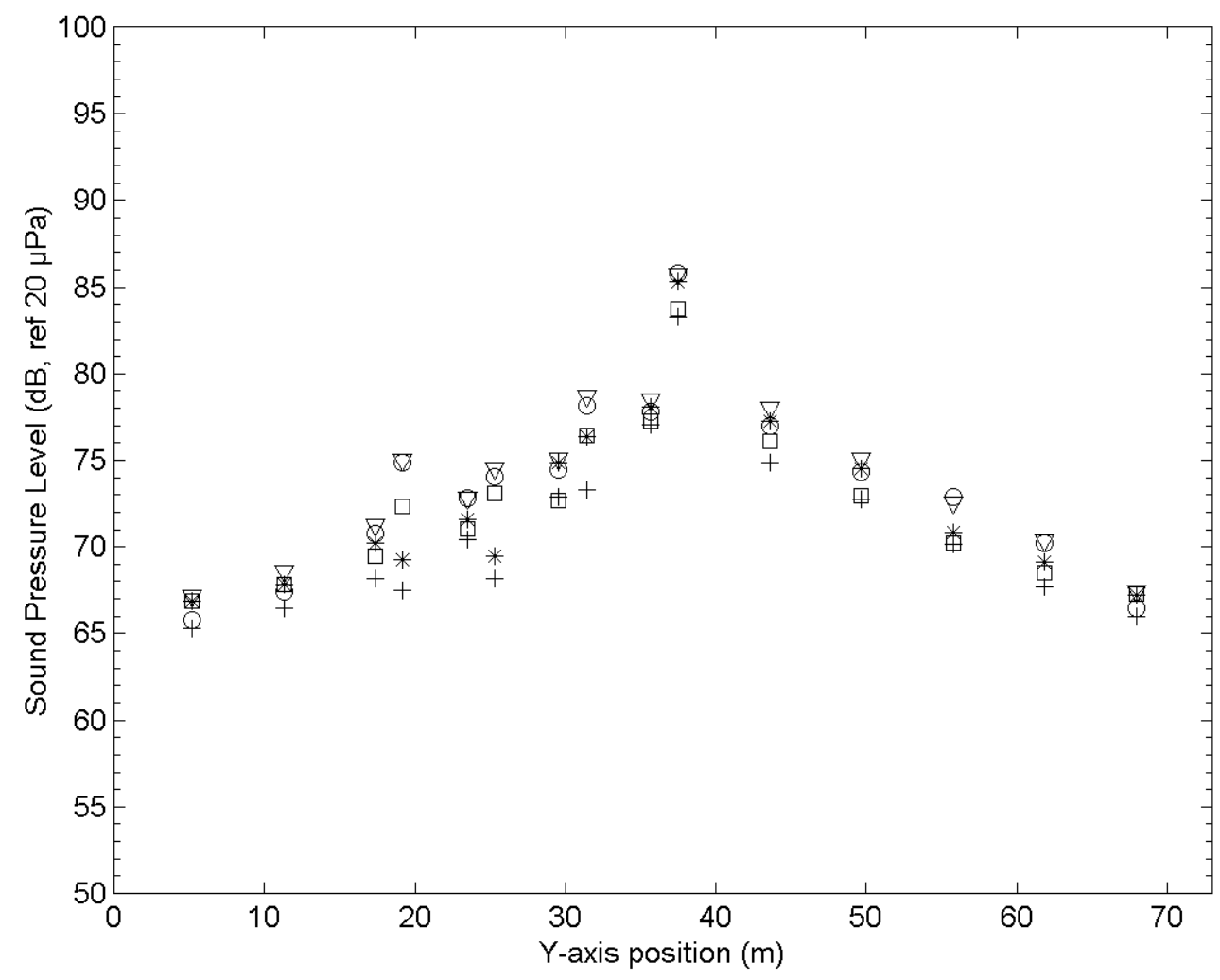

Figure B.37: Overall sound pressure level for sources 41 and 42 as a function of window location. $\nabla$ level of existing toll plaza; * level with absorbing canopy; O level with absorbing barriers; level with absorbing barriers; + level is all modifications. 


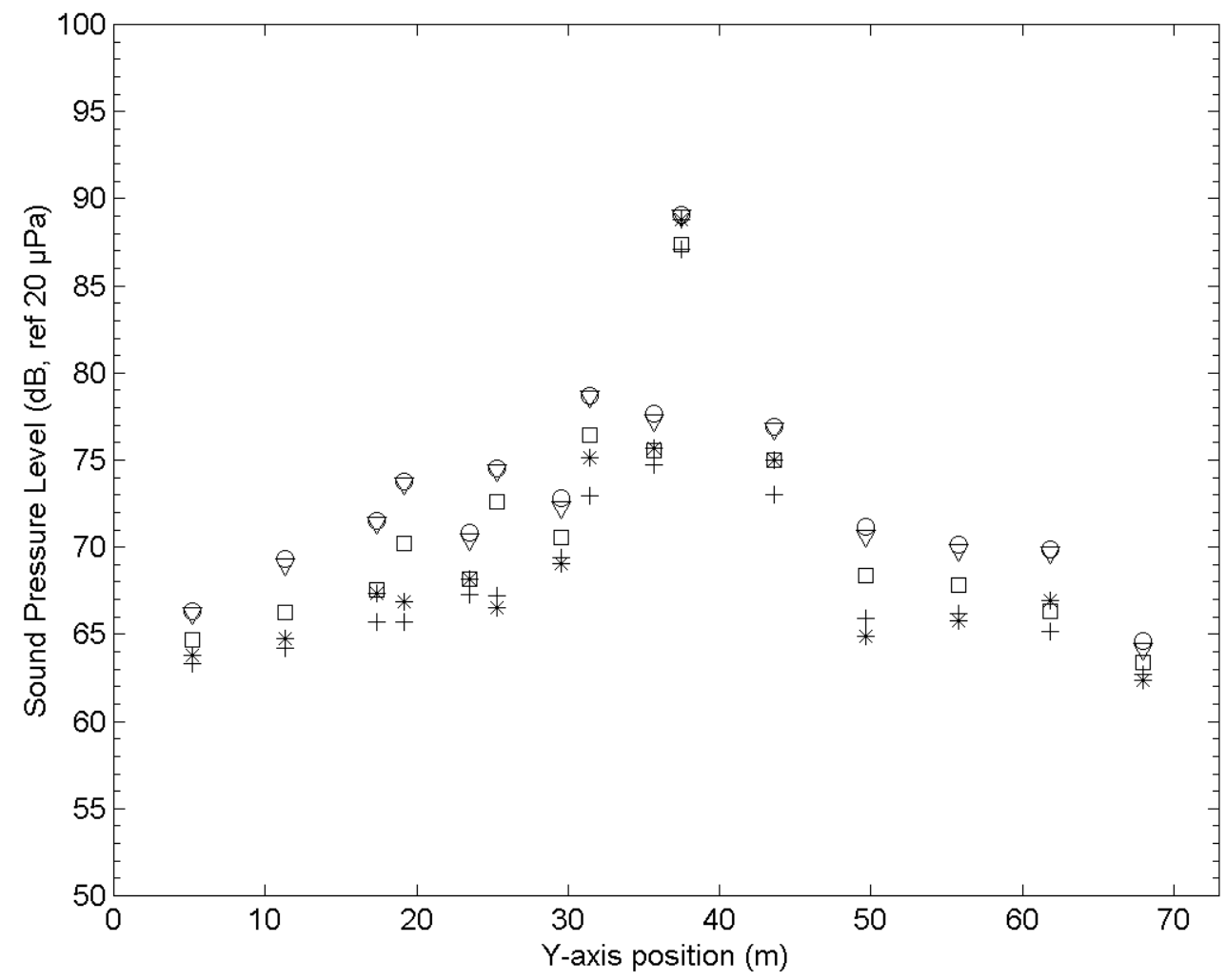

Figure B.38: Overall sound pressure level for sources 43 and 44 as a function of window location. $\nabla$ level of existing toll plaza; * level with absorbing canopy; $\mathrm{O}$ level with absorbing barriers; level with absorbing barriers; + level is all modifications. 


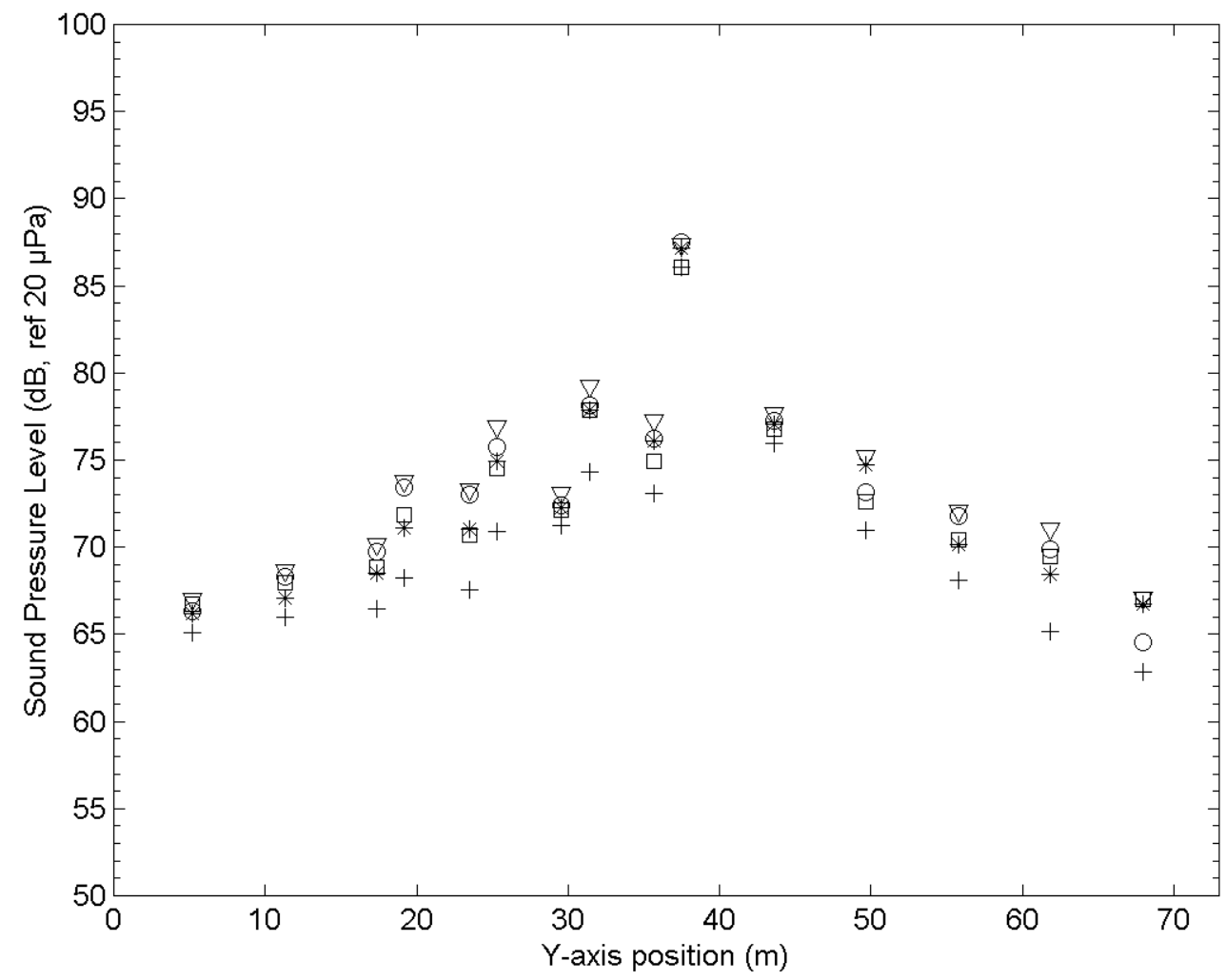

Figure B.39: Overall sound pressure level for sources 45 and 46 as a function of window location. $\nabla$ level of existing toll plaza; * level with absorbing canopy; O level with absorbing barriers; level with absorbing barriers; + level is all modifications. 


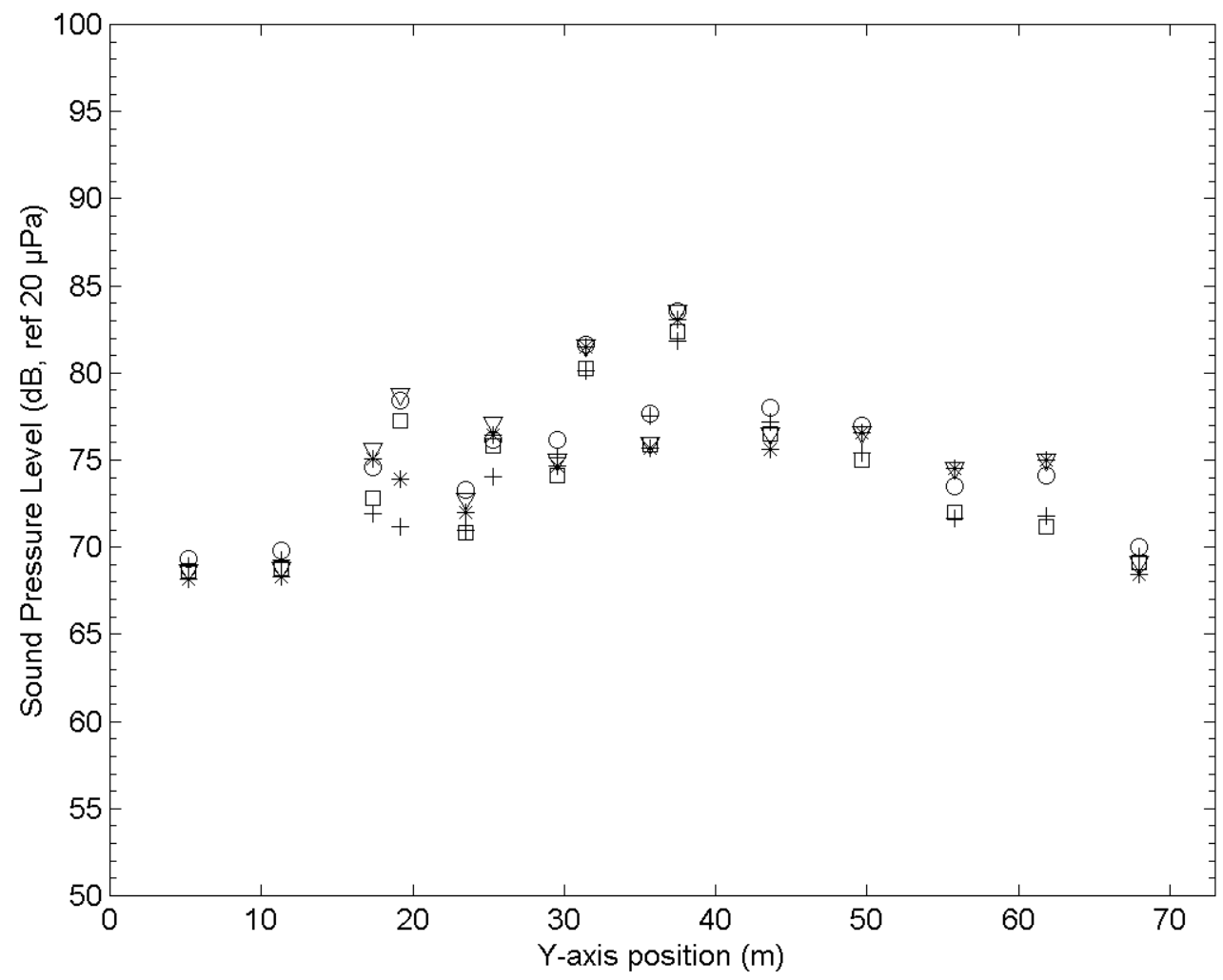

Figure B.40: Overall sound pressure level for sources 47 and 48 as a function of window location. $\nabla$ level of existing toll plaza; * level with absorbing canopy; $\mathrm{O}$ level with absorbing barriers; level with absorbing barriers; + level is all modifications. 


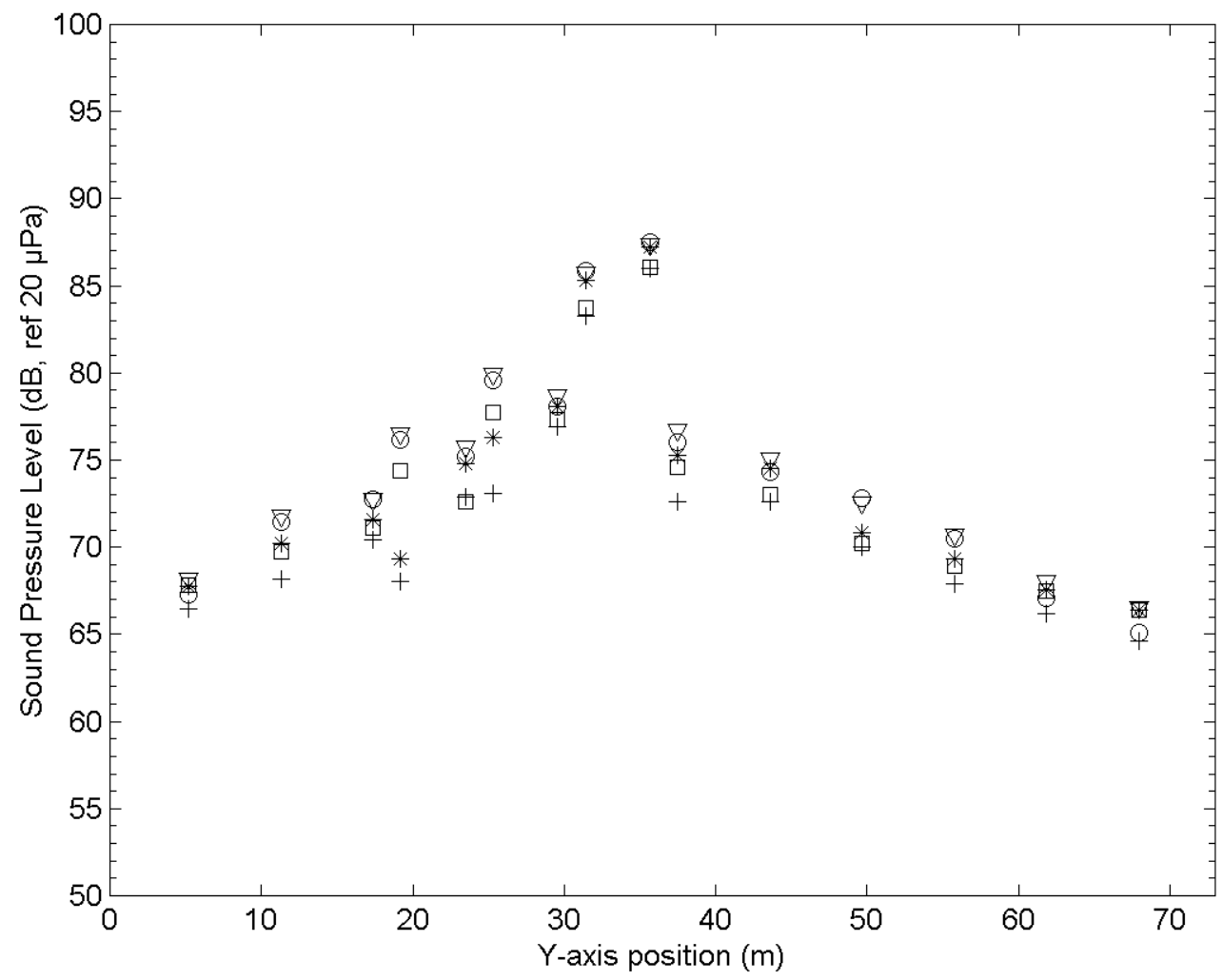

Figure B.41: Overall sound pressure level for sources 49 and 50 as a function of window location. $\nabla$ level of existing toll plaza; * level with absorbing canopy; $\mathrm{O}$ level with absorbing barriers; level with absorbing barriers; + level is all modifications. 


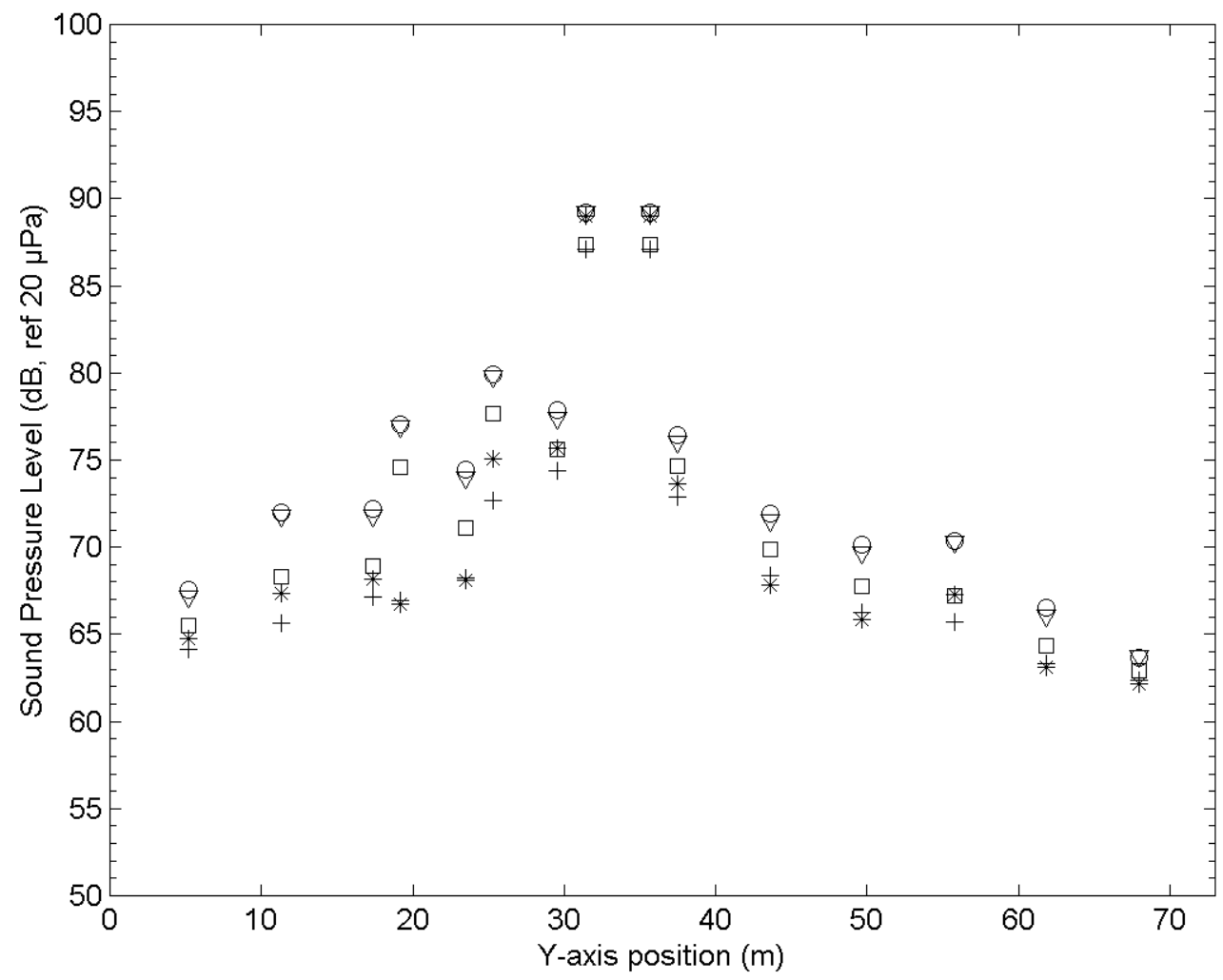

Figure B.42: Overall sound pressure level for sources 51 and 52 as a function of window location. $\nabla$ level of existing toll plaza; * level with absorbing canopy; $\mathrm{O}$ level with absorbing barriers; level with absorbing barriers; + level is all modifications. 


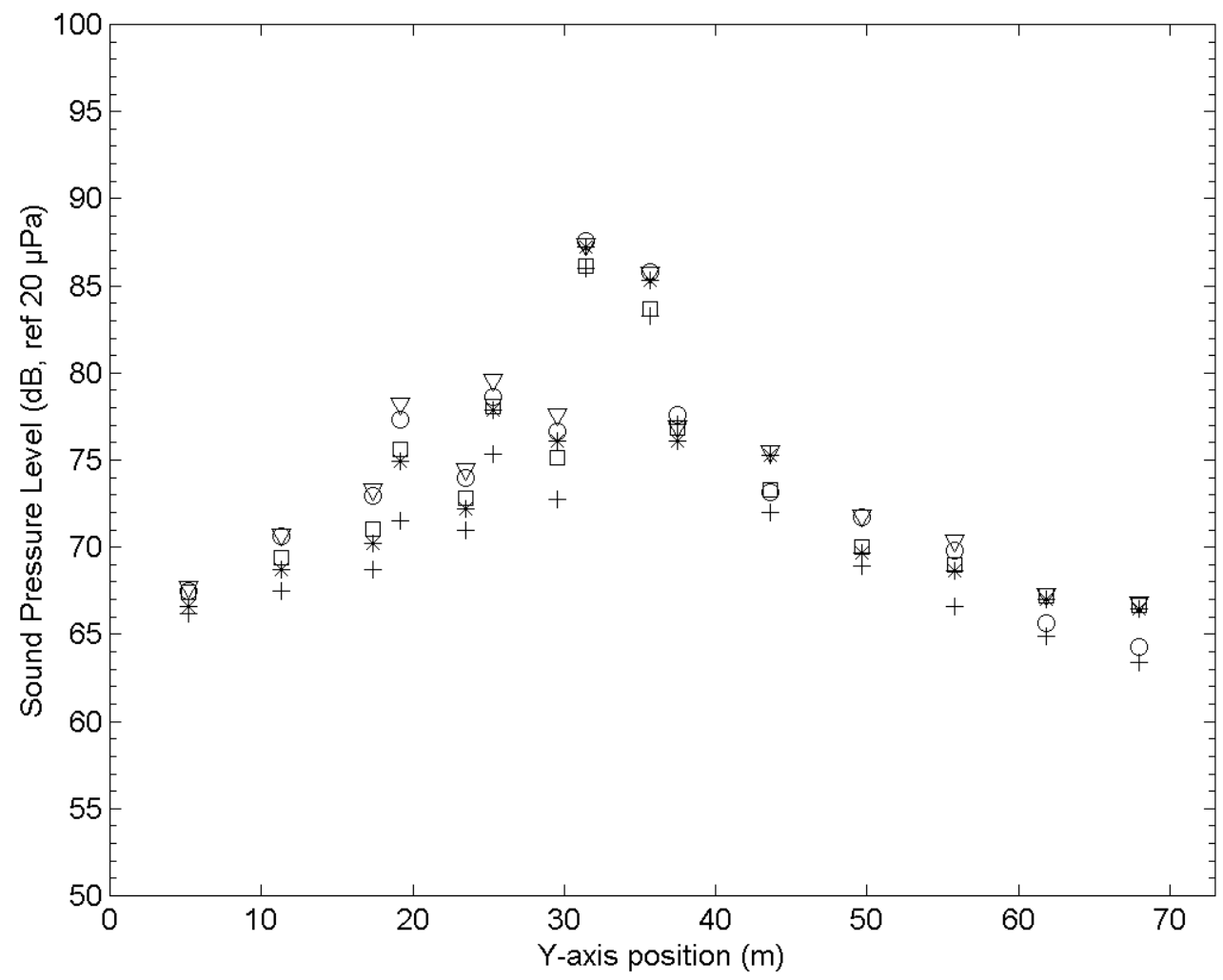

Figure B.43: Overall sound pressure level for sources 53 and 54 as a function of window location. $\nabla$ level of existing toll plaza; * level with absorbing canopy; $\mathrm{O}$ level with absorbing barriers; level with absorbing barriers; + level is all modifications. 


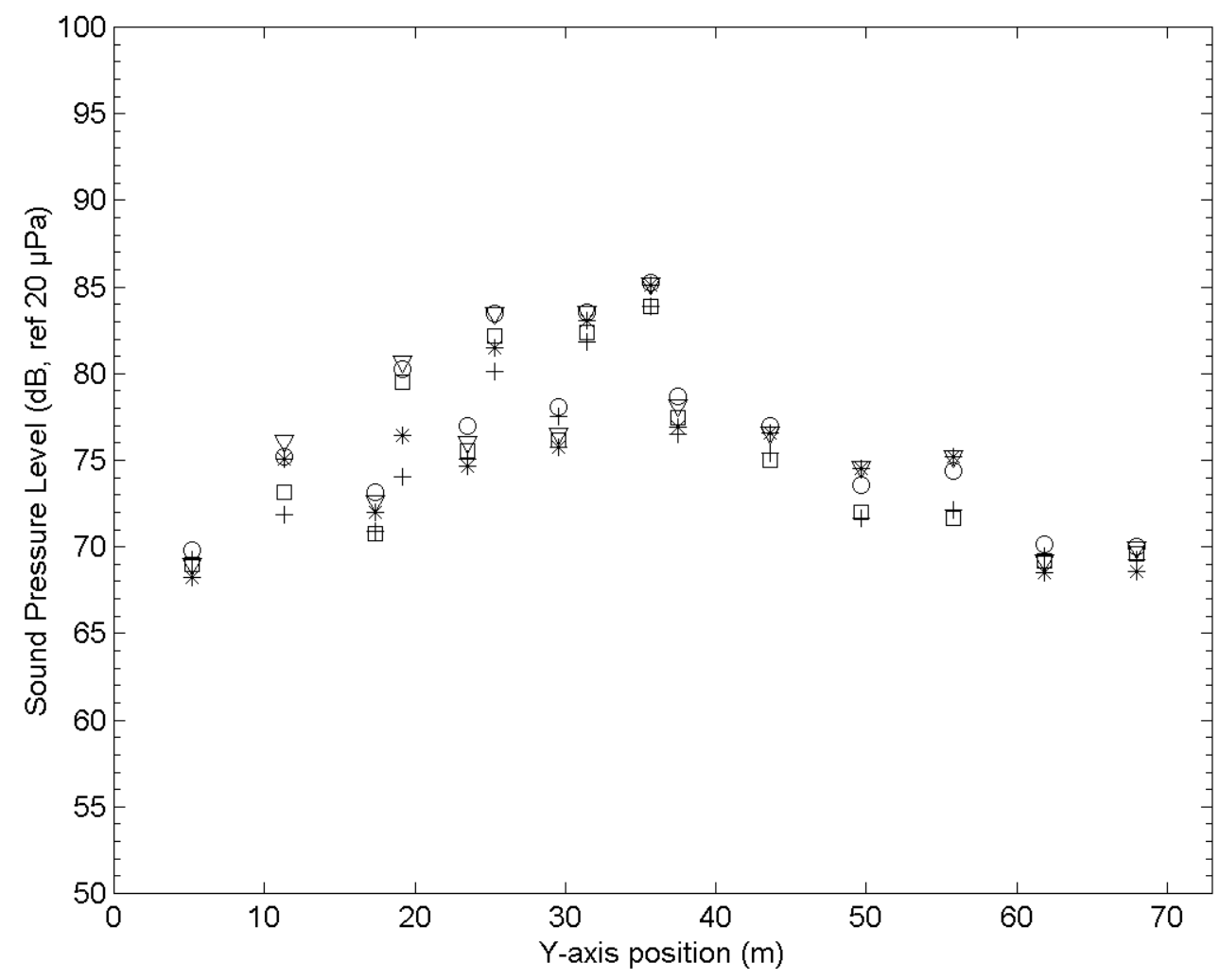

Figure B.44: Overall sound pressure level for sources 55 and 56 as a function of window location. $\nabla$ level of existing toll plaza; * level with absorbing canopy; $\mathrm{O}$ level with absorbing barriers; level with absorbing barriers; + level is all modifications. 


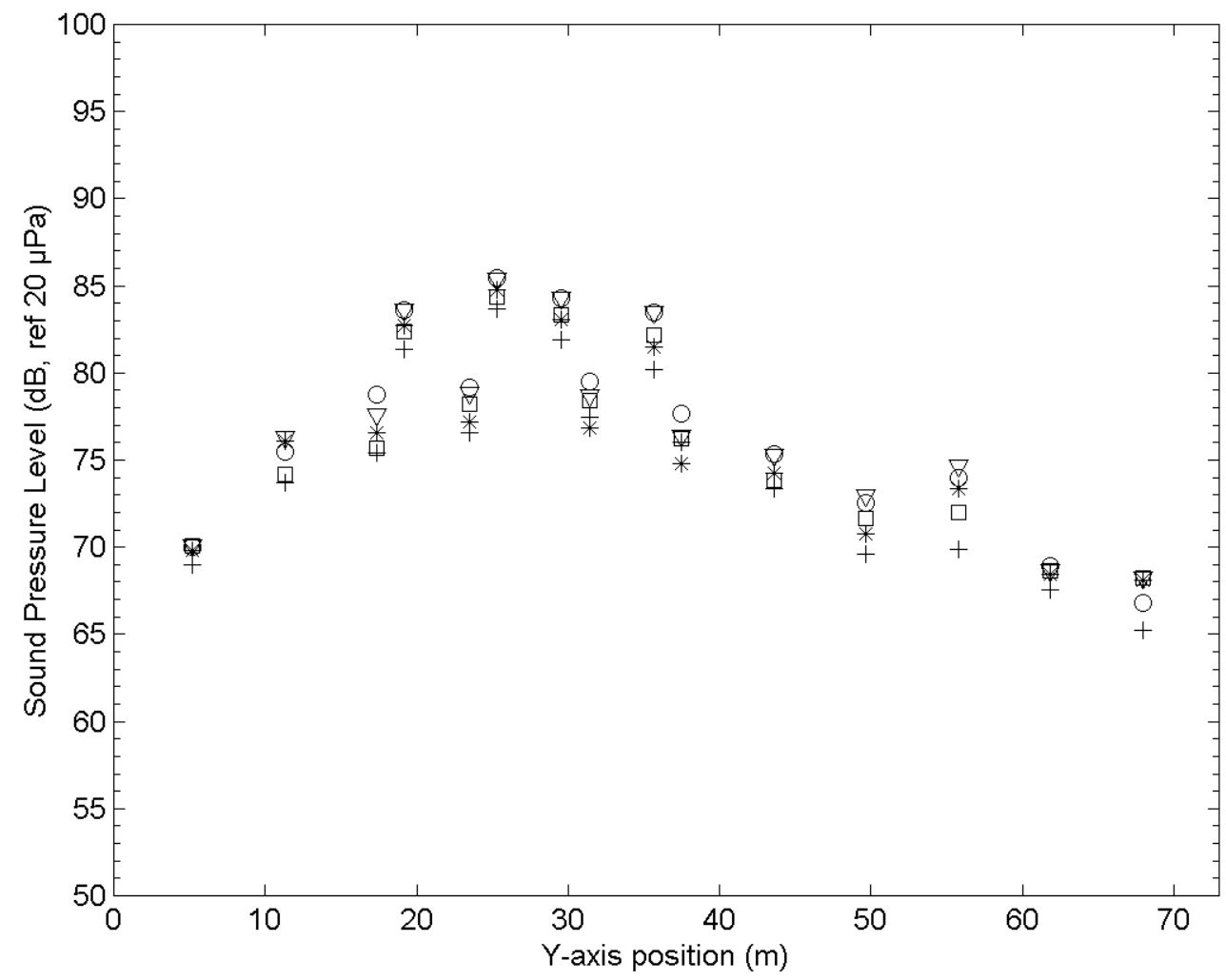

Figure B.45: Overall sound pressure level for sources 57 and 58 as a function of window location. $\nabla$ level of existing toll plaza; * level with absorbing canopy; $\mathrm{O}$ level with absorbing barriers; level with absorbing barriers; + level is all modifications. 


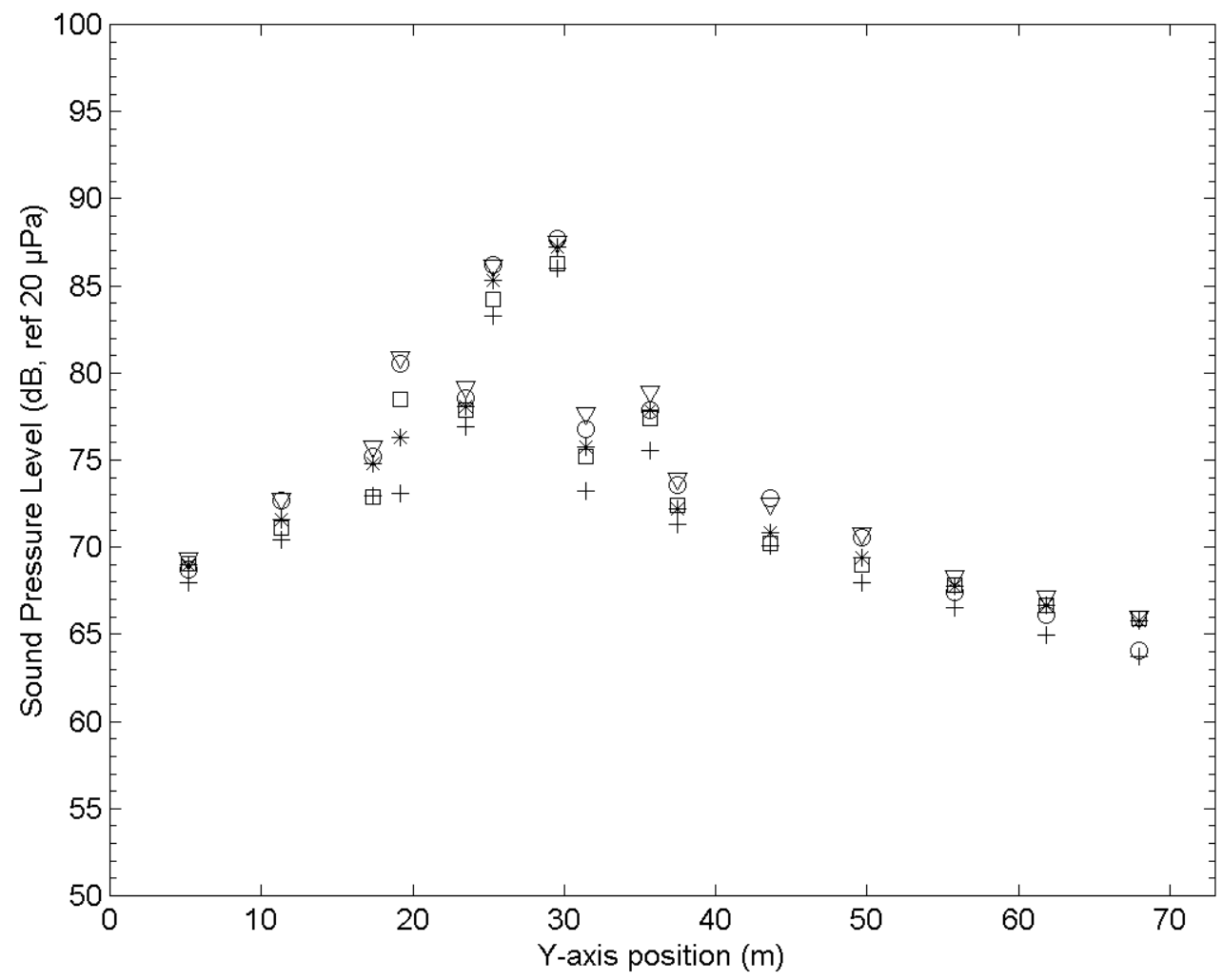

Figure B.46: Overall sound pressure level for sources 59 and 60 as a function of window location. $\nabla$ level of existing toll plaza; * level with absorbing canopy; $\mathrm{O}$ level with absorbing barriers; level with absorbing barriers; + level is all modifications. 


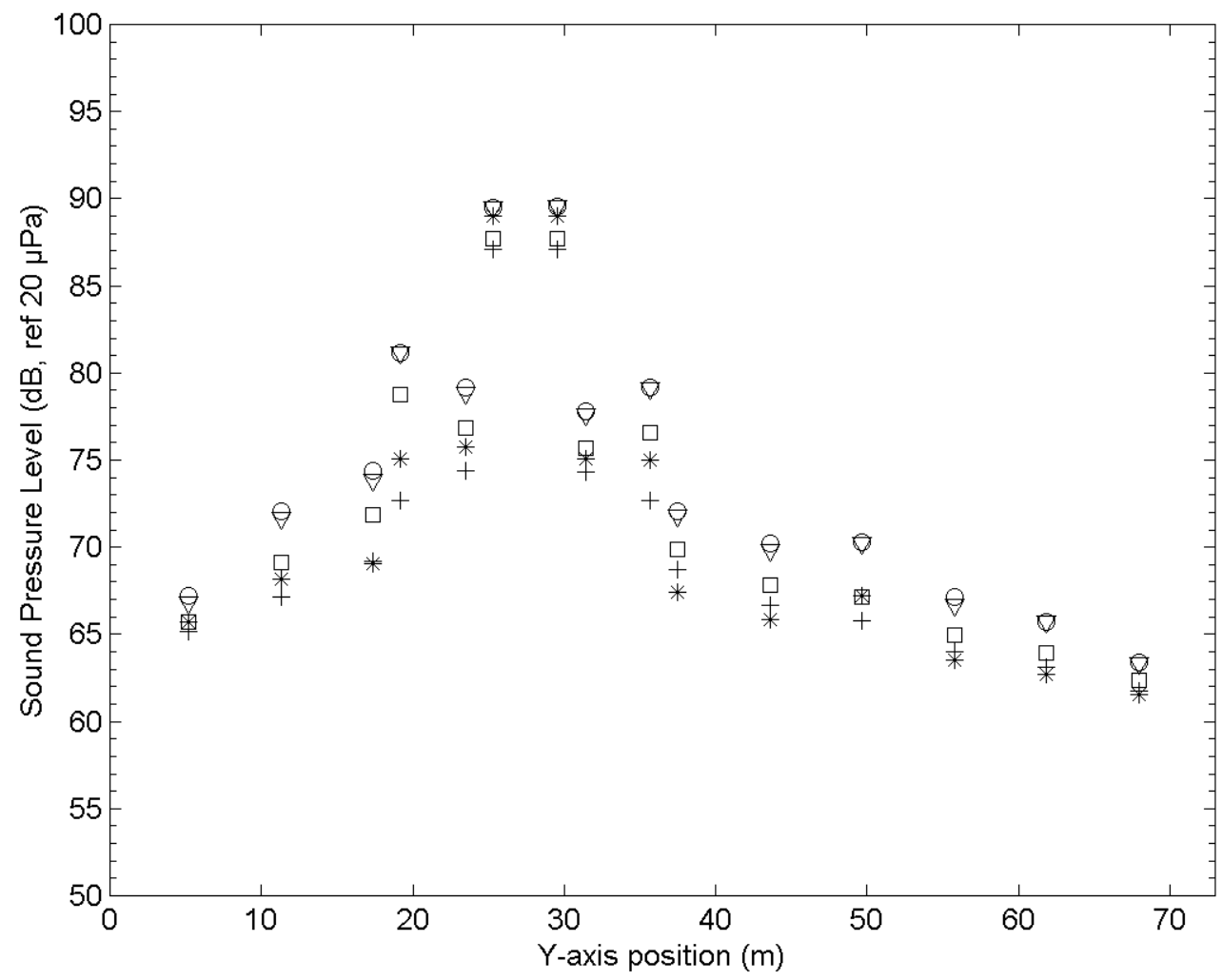

Figure B.47: Overall sound pressure level for sources 61 and 62 as a function of window location. $\nabla$ level of existing toll plaza; * level with absorbing canopy; $\mathrm{O}$ level with absorbing barriers; level with absorbing barriers; + level is all modifications. 


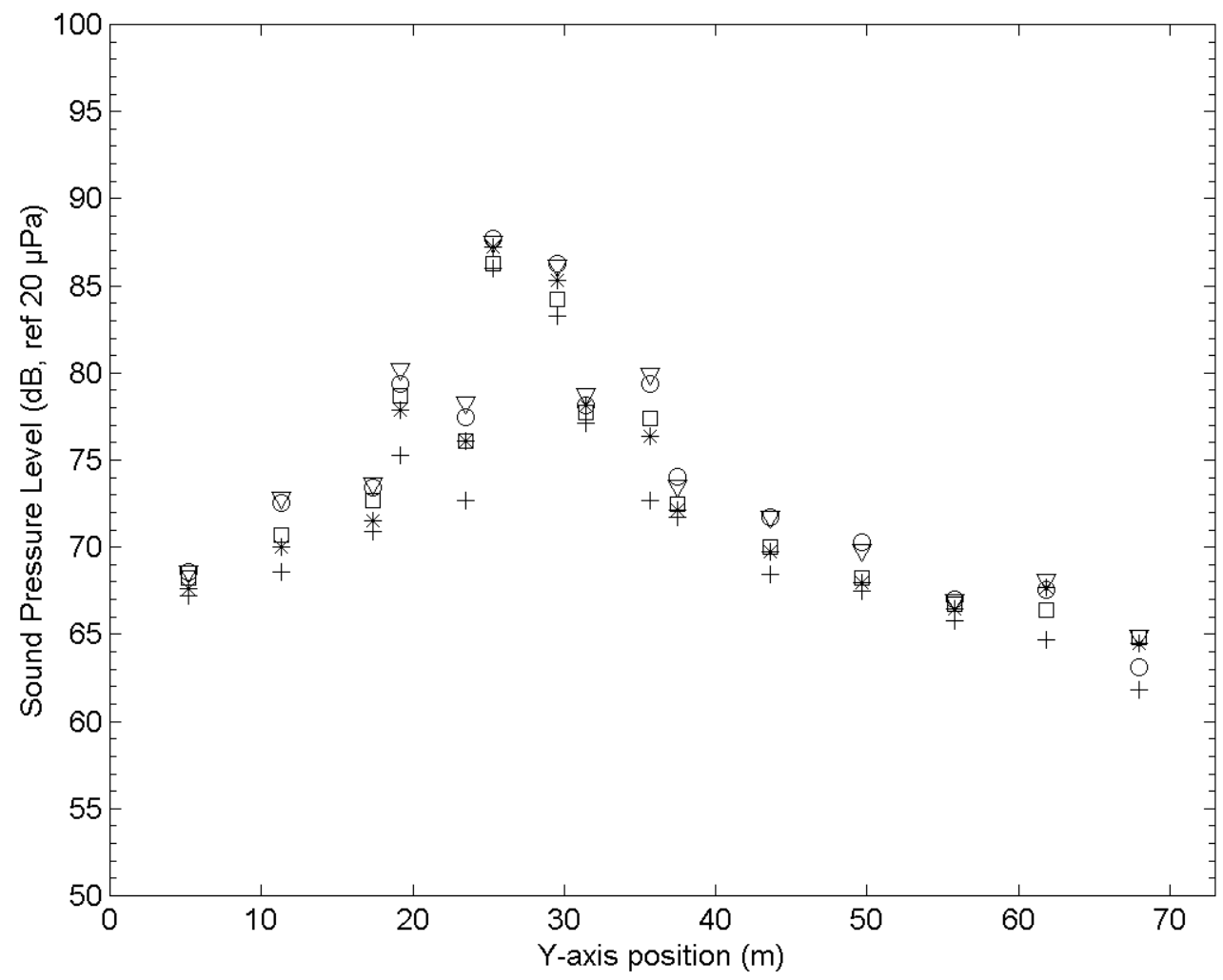

Figure B.48: Overall sound pressure level for sources 63 and 64 as a function of window location. $\nabla$ level of existing toll plaza; * level with absorbing canopy; $\mathrm{O}$ level with absorbing barriers; level with absorbing barriers; + level is all modifications. 


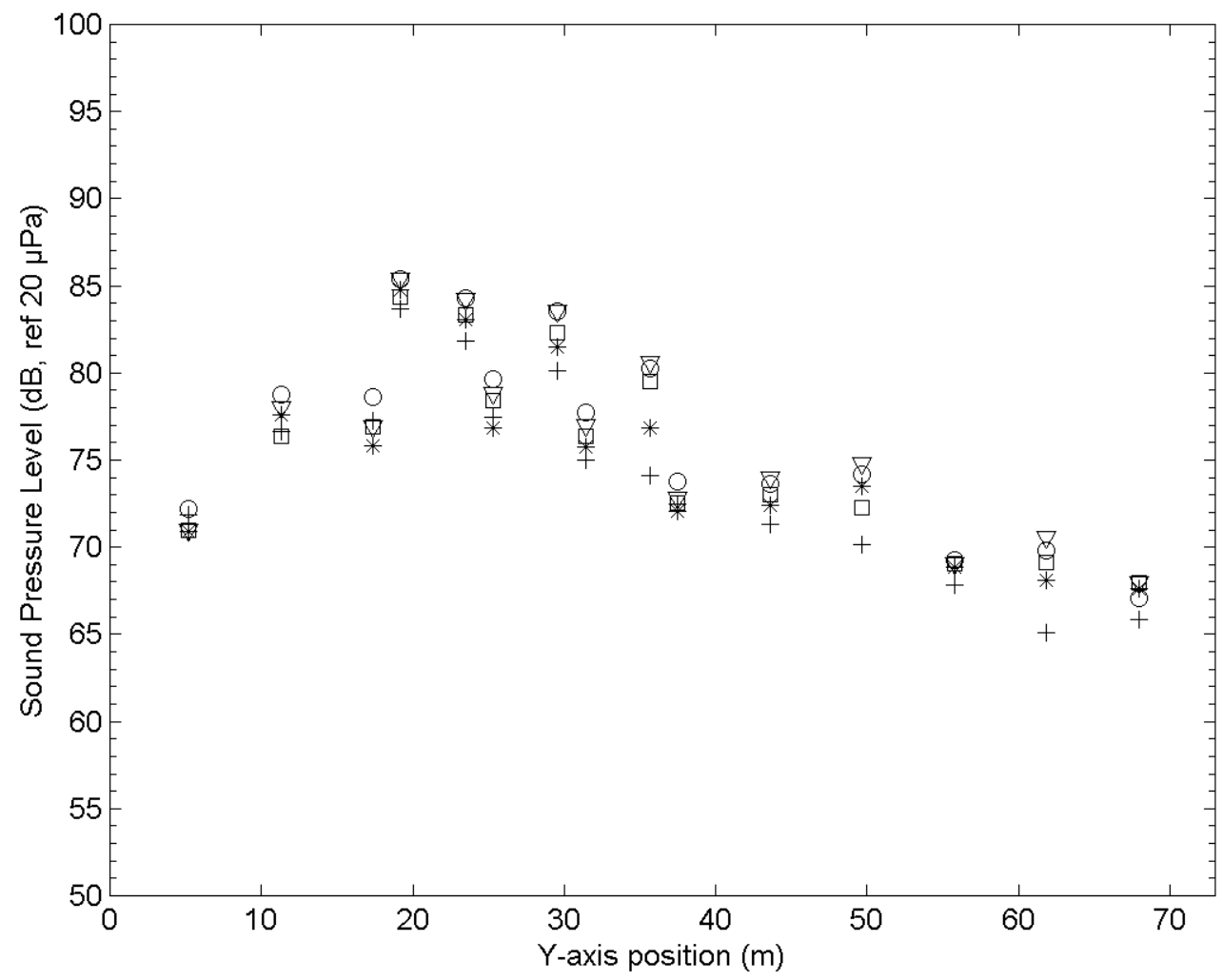

Figure B.49: Overall sound pressure level for sources 65 and 66 as a function of window location. $\nabla$ level of existing toll plaza; * level with absorbing canopy; $\mathrm{O}$ level with absorbing barriers; level with absorbing barriers; + level is all modifications. 


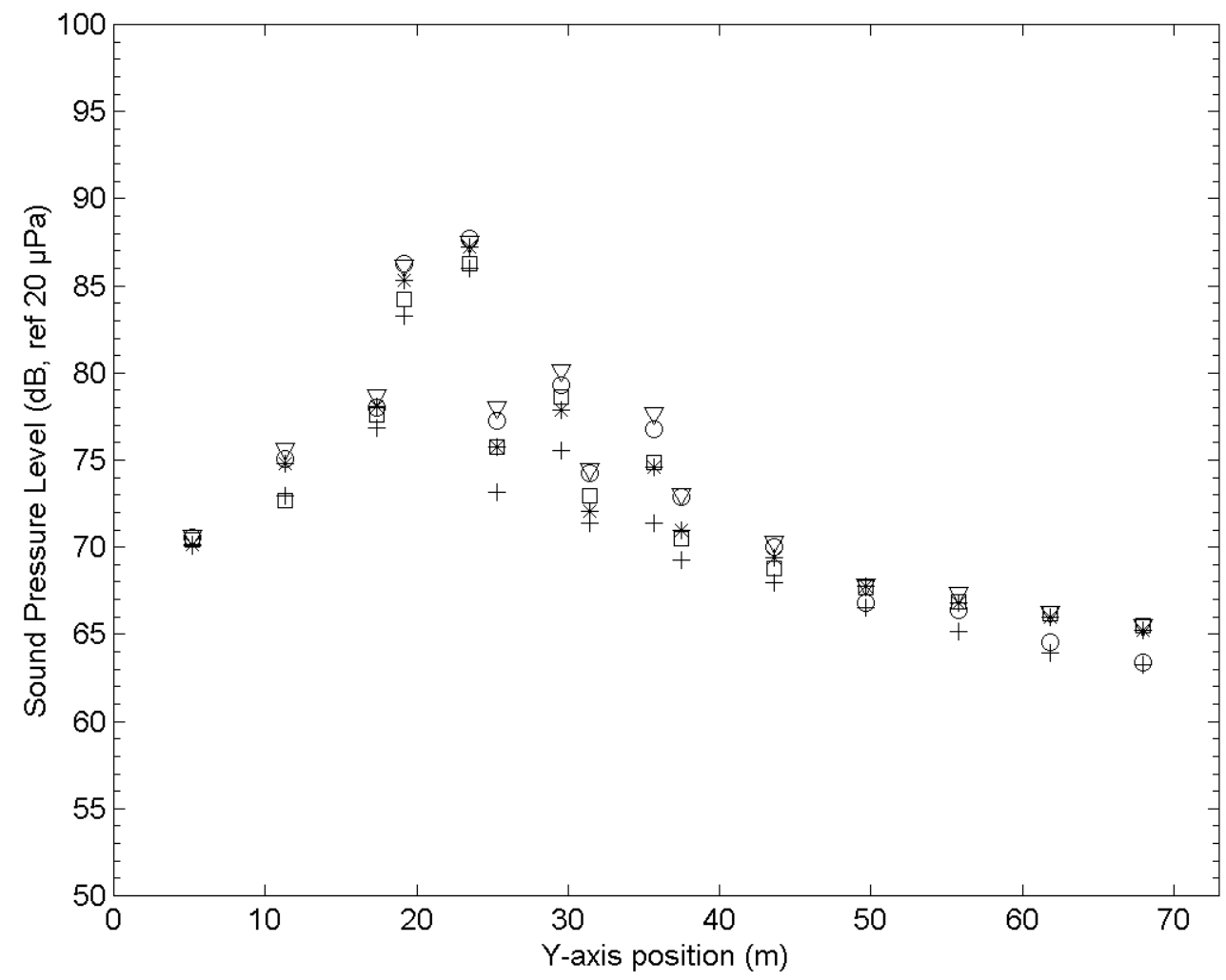

Figure B.50: Overall sound pressure level for sources 67 and 68 as a function of window location. $\nabla$ level of existing toll plaza; * level with absorbing canopy; $\mathrm{O}$ level with absorbing barriers; level with absorbing barriers; + level is all modifications. 


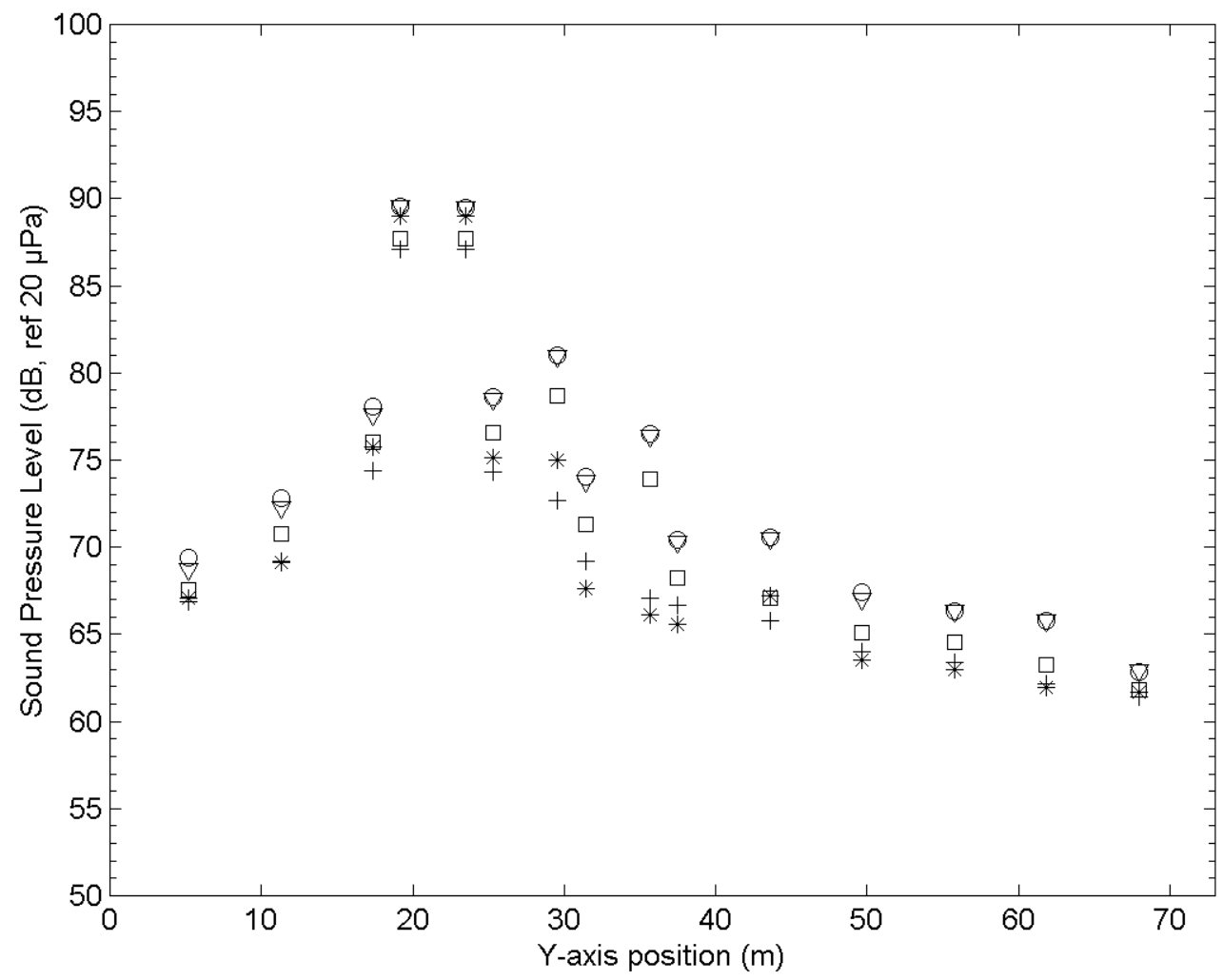

Figure B.51: Overall sound pressure level for sources 69 and 70 as a function of window location. $\nabla$ level of existing toll plaza; * level with absorbing canopy; $\mathrm{O}$ level with absorbing barriers; level with absorbing barriers; + level is all modifications. 


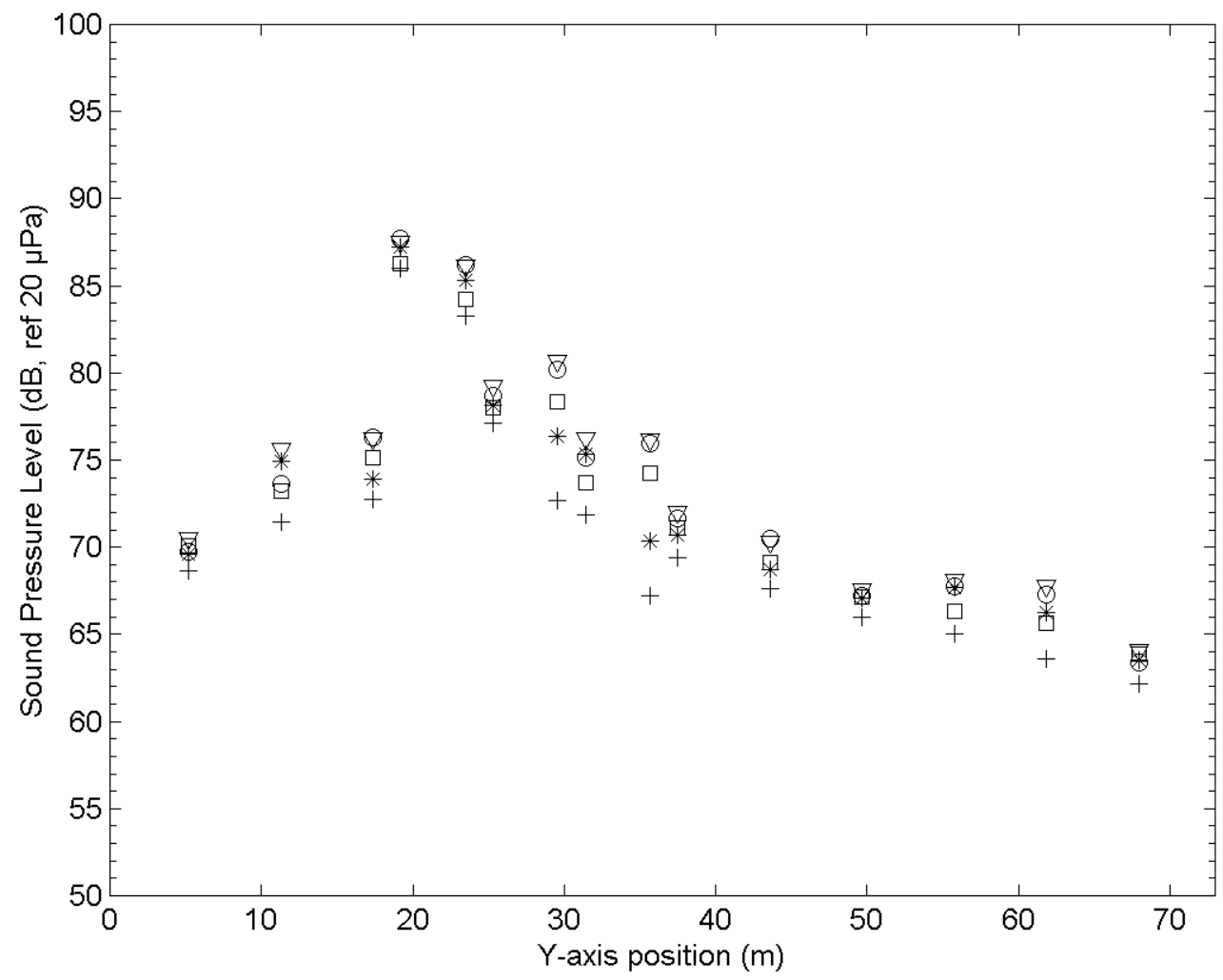

Figure B.52: Overall sound pressure level for sources 71 and 72 as a function of window location. $\nabla$ level of existing toll plaza; * level with absorbing canopy; $\mathrm{O}$ level with absorbing barriers; level with absorbing barriers; + level is all modifications. 


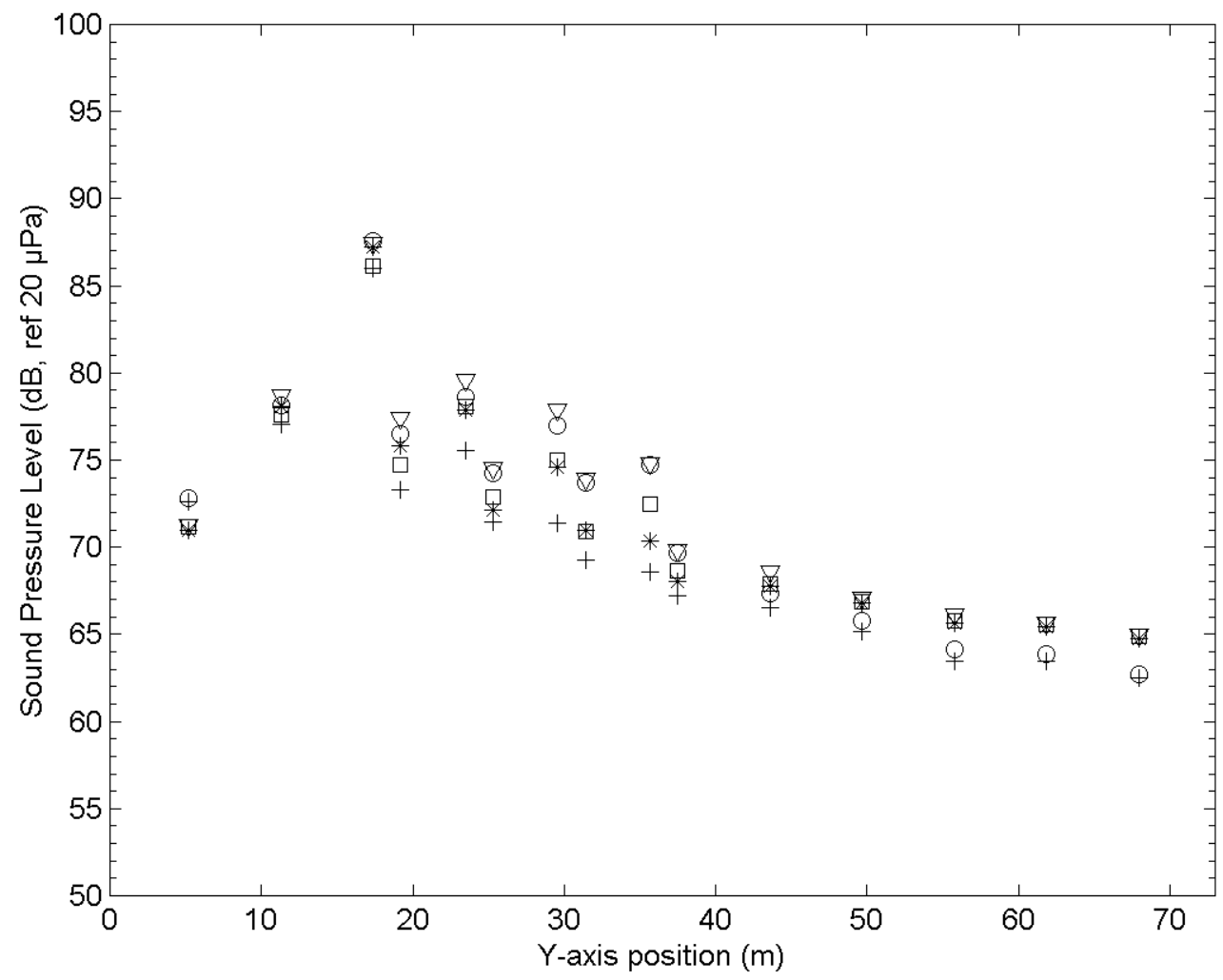

Figure B.53: Overall sound pressure level for sources 73 and 74 as a function of window location. $\nabla$ level of existing toll plaza; * level with absorbing canopy; O level with absorbing barriers; level with absorbing barriers; + level is all modifications. 


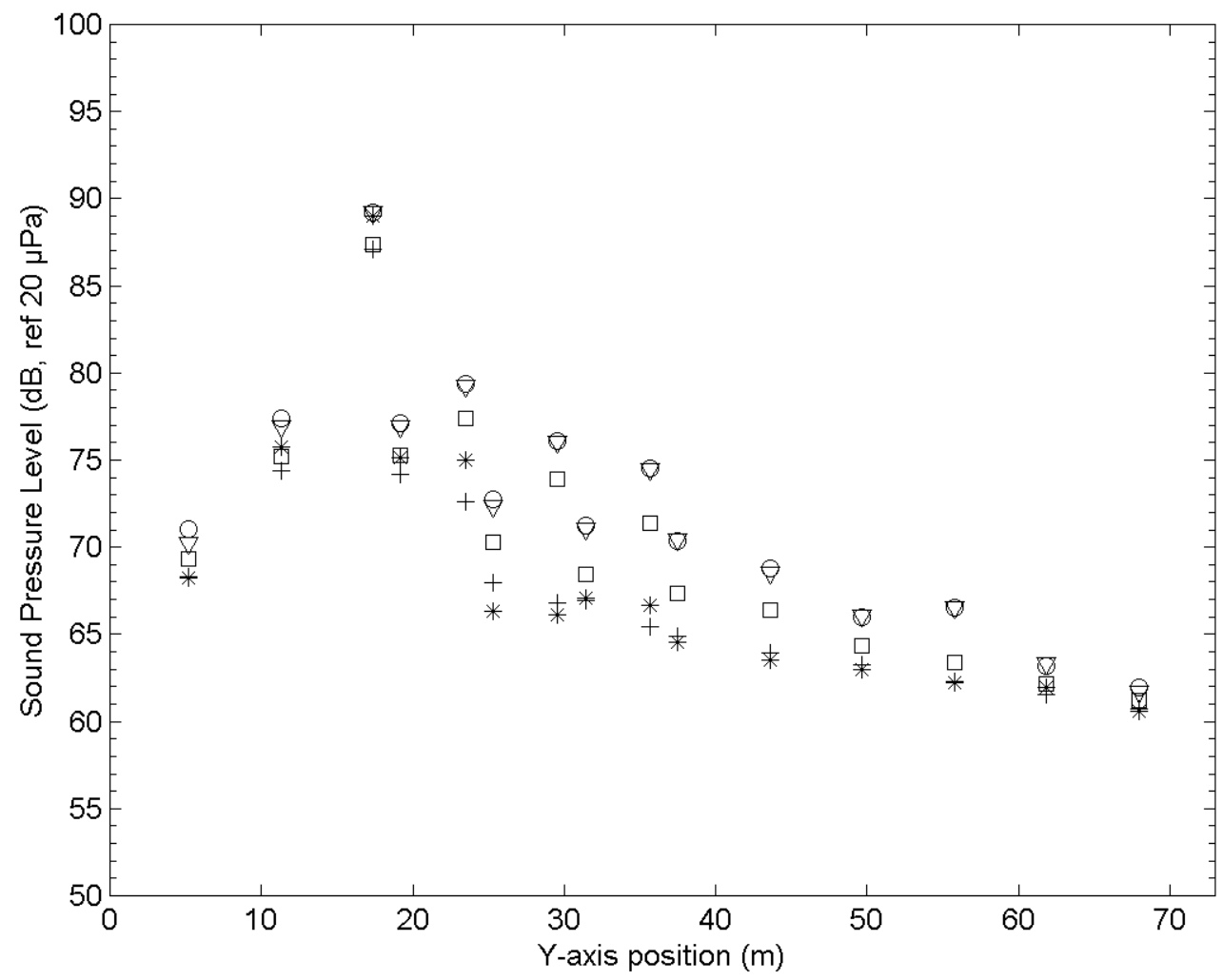

Figure B.54: Overall sound pressure level for sources 75 and 76 as a function of window location. $\nabla$ level of existing toll plaza; * level with absorbing canopy; $\mathrm{O}$ level with absorbing barriers; level with absorbing barriers; + level is all modifications. 


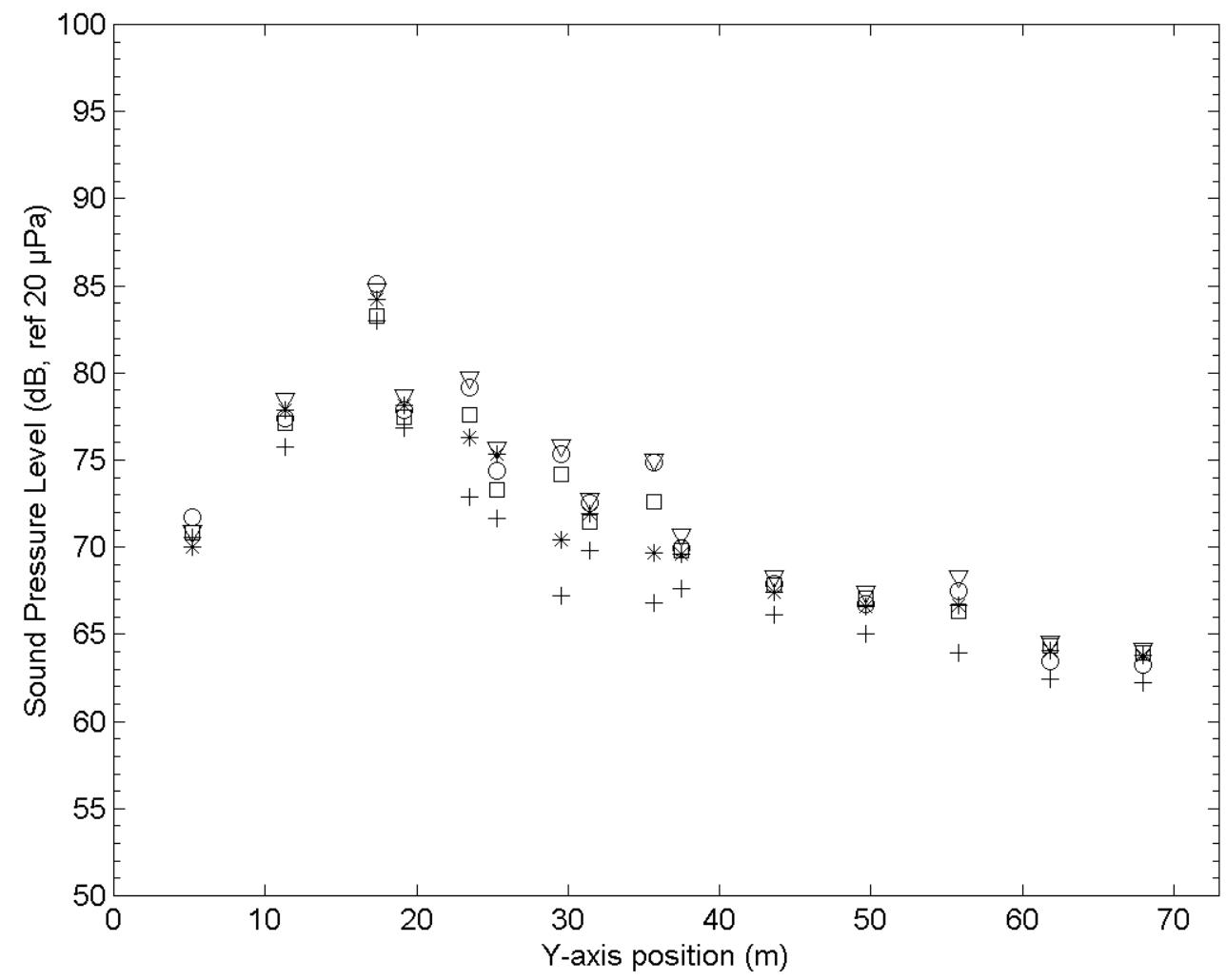

Figure B.55: Overall sound pressure level for sources 77 and 78 as a function of window location. $\nabla$ level of existing toll plaza; * level with absorbing canopy; $\mathrm{O}$ level with absorbing barriers; level with absorbing barriers; + level is all modifications. 


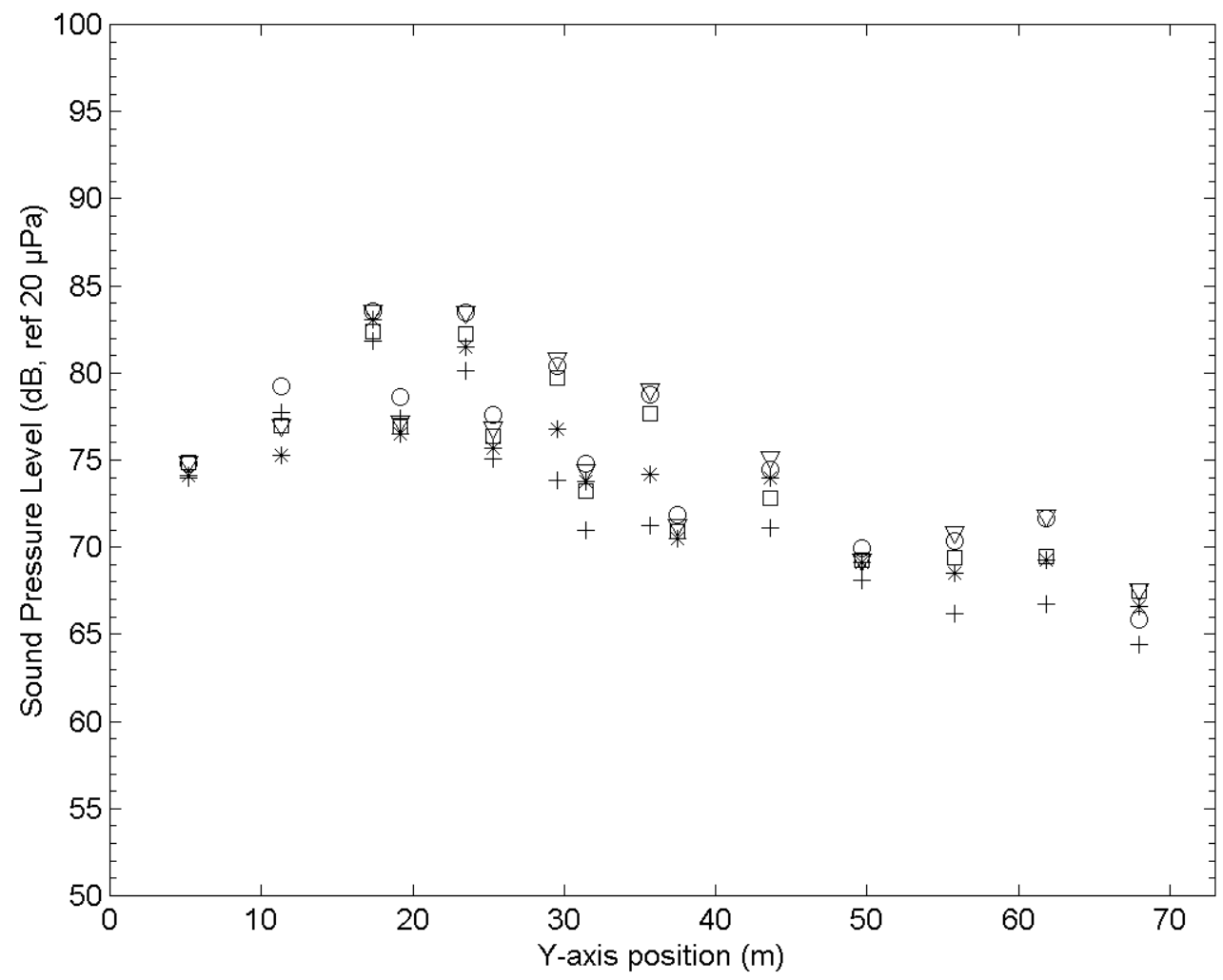

Figure B.56: Overall sound pressure level for sources 79 and 80 as a function of window location. $\nabla$ level of existing toll plaza; * level with absorbing canopy; $\mathrm{O}$ level with absorbing barriers; level with absorbing barriers; + level is all modifications. 


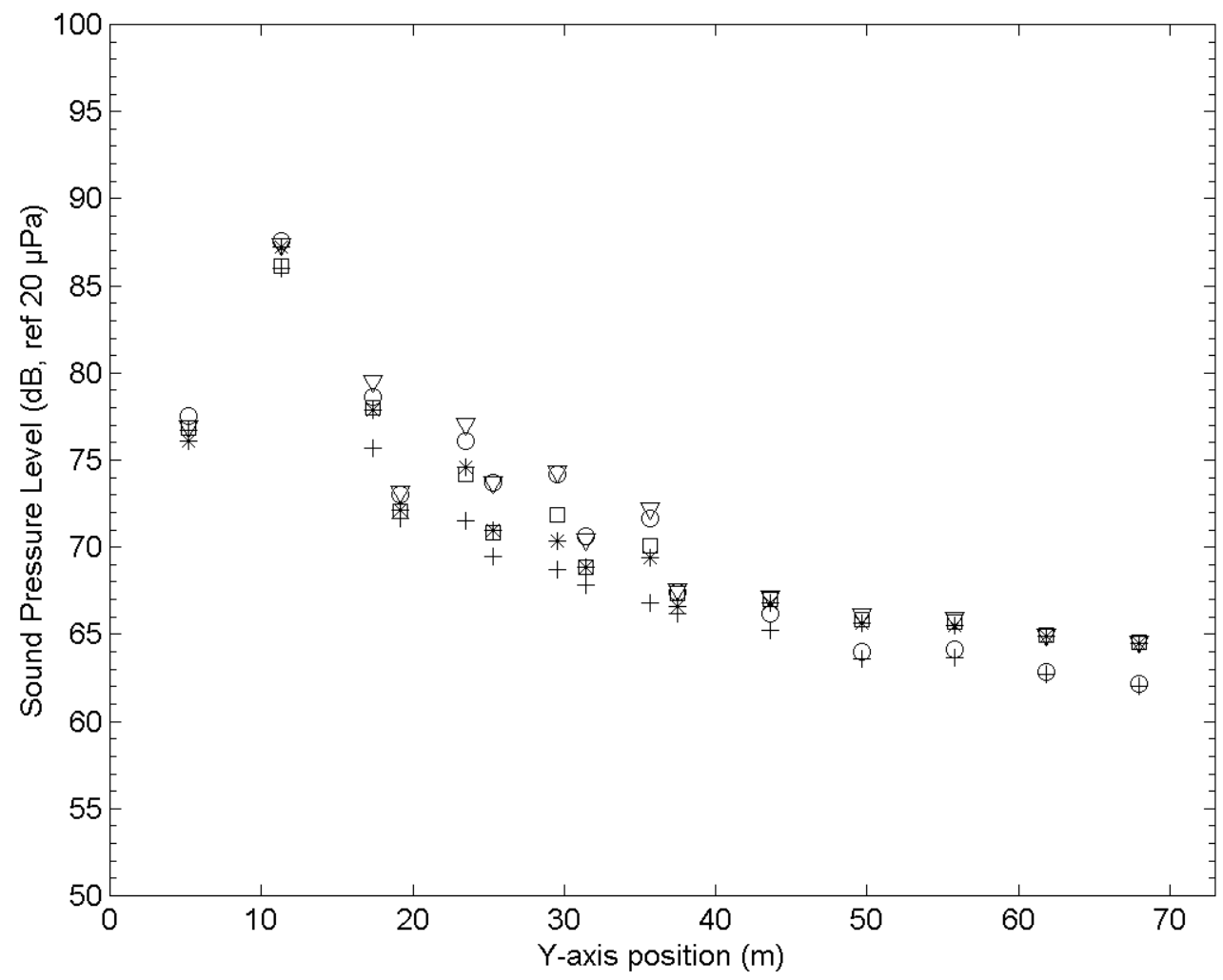

Figure B.57: Overall sound pressure level for sources 81 and 82 as a function of window location. $\nabla$ level of existing toll plaza; * level with absorbing canopy; O level with absorbing barriers; level with absorbing barriers; + level is all modifications. 


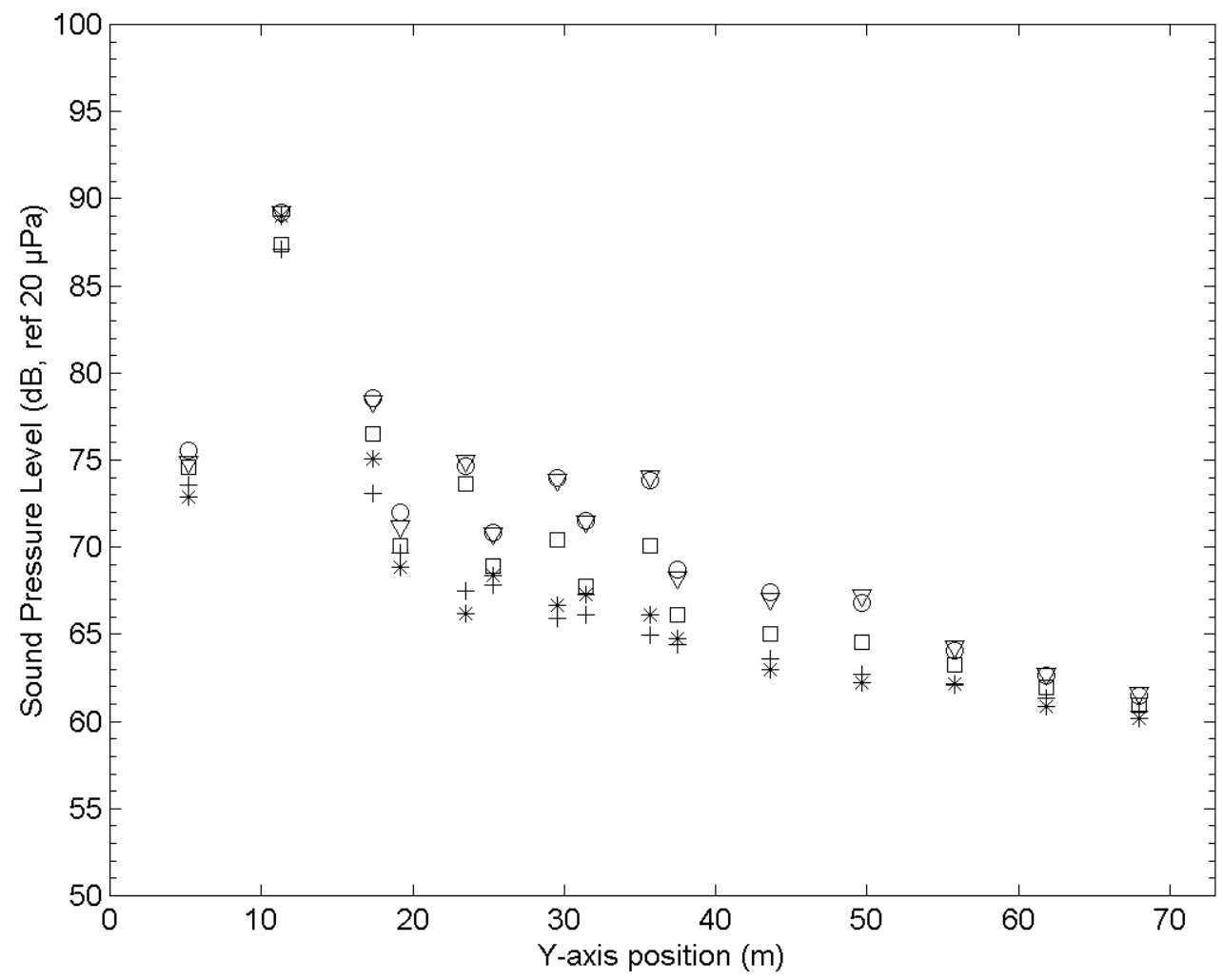

Figure B.58: Overall sound pressure level for sources 83 and 84 as a function of window location. $\nabla$ level of existing toll plaza; * level with absorbing canopy; $\mathrm{O}$ level with absorbing barriers; level with absorbing barriers; + level is all modifications. 


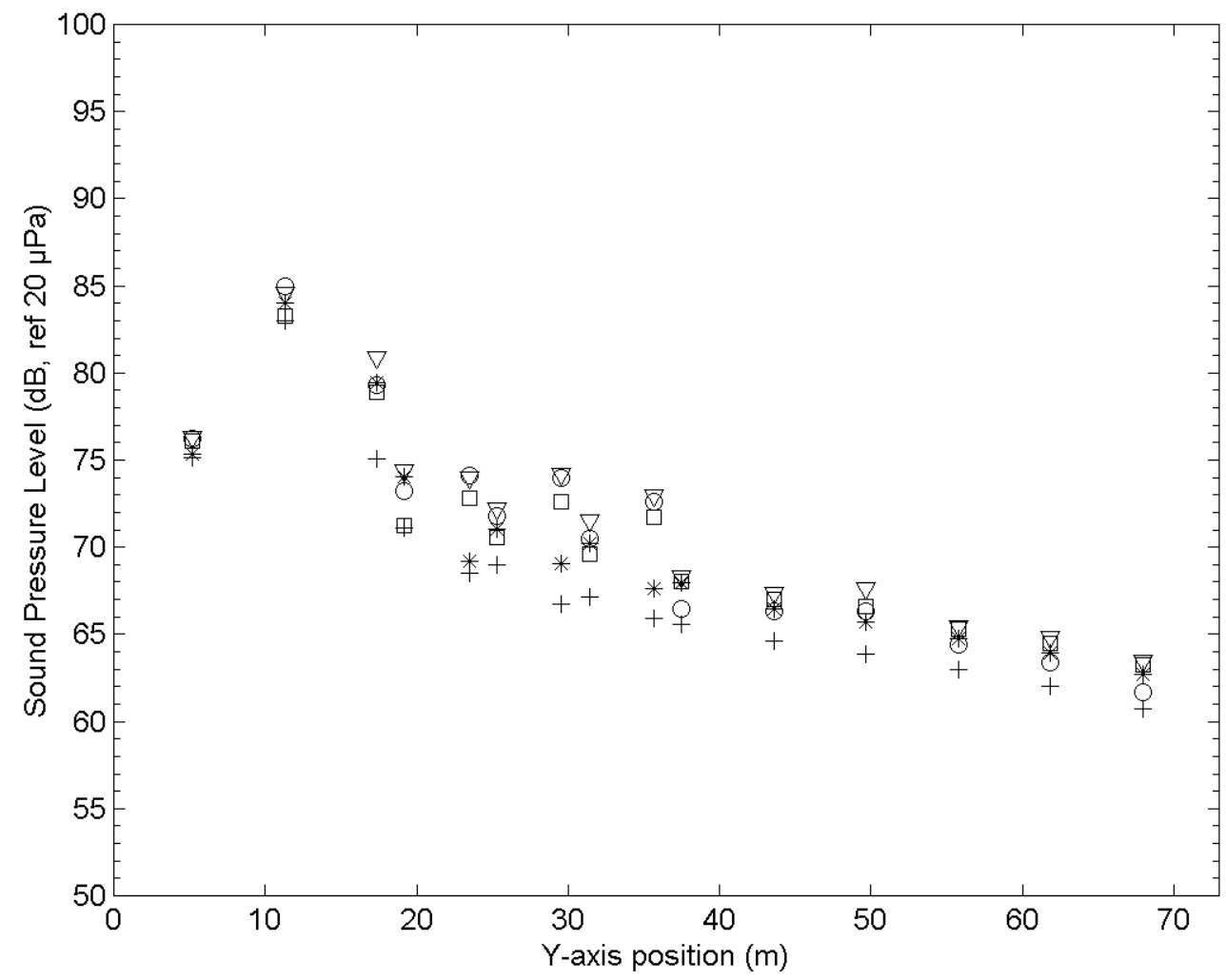

Figure B.59: Overall sound pressure level for sources 85 and 86 as a function of window location. $\nabla$ level of existing toll plaza; * level with absorbing canopy; O level with absorbing barriers; level with absorbing barriers; + level is all modifications. 


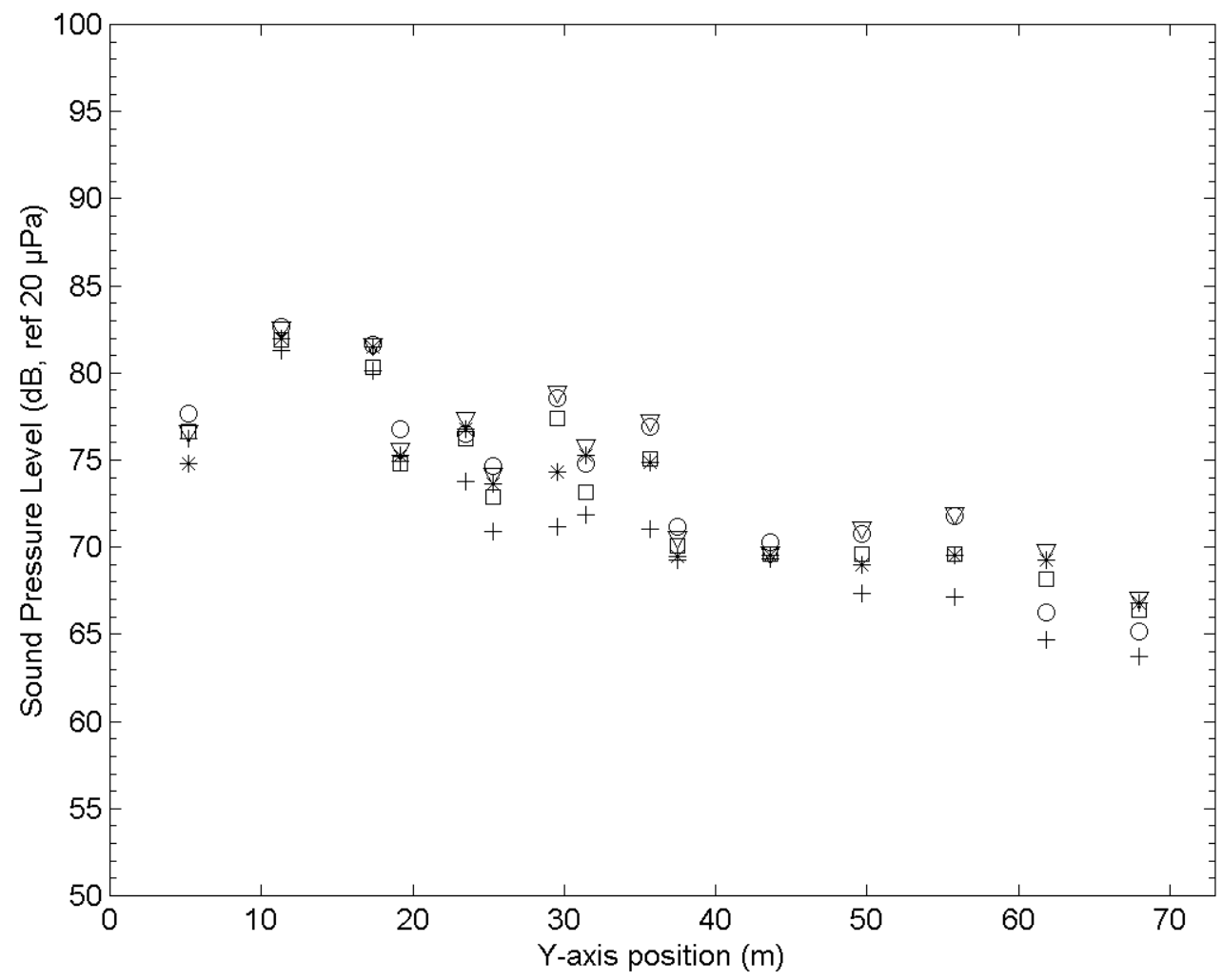

Figure B.60: Overall sound pressure level for sources 87 and 88 as a function of window location. $\nabla$ level of existing toll plaza; * level with absorbing canopy; $\mathrm{O}$ level with absorbing barriers; level with absorbing barriers; + level is all modifications. 


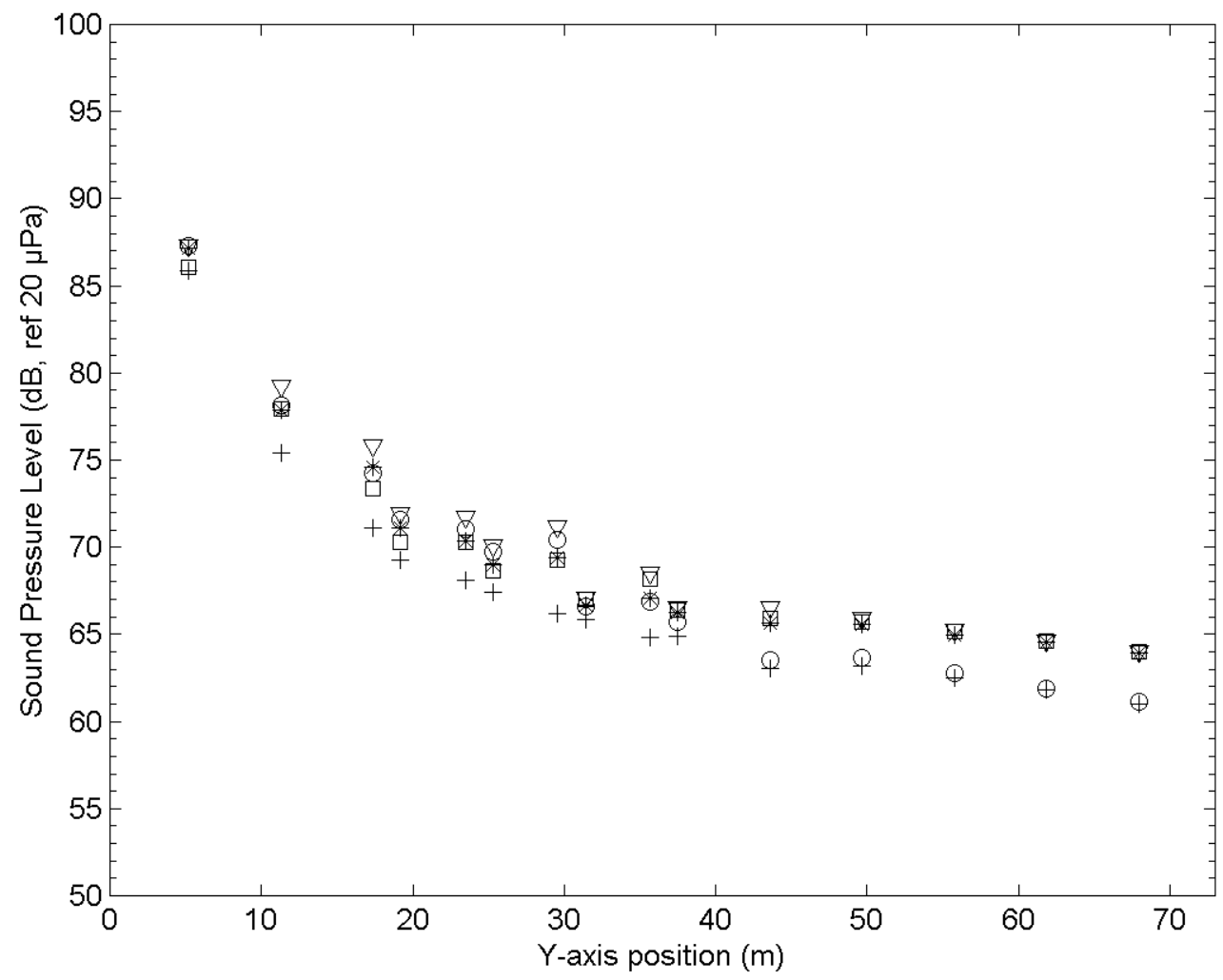

Figure B.61: Overall sound pressure level for sources 89 and 90 as a function of window location. $\nabla$ level of existing toll plaza; * level with absorbing canopy; O level with absorbing barriers; level with absorbing barriers; + level is all modifications. 


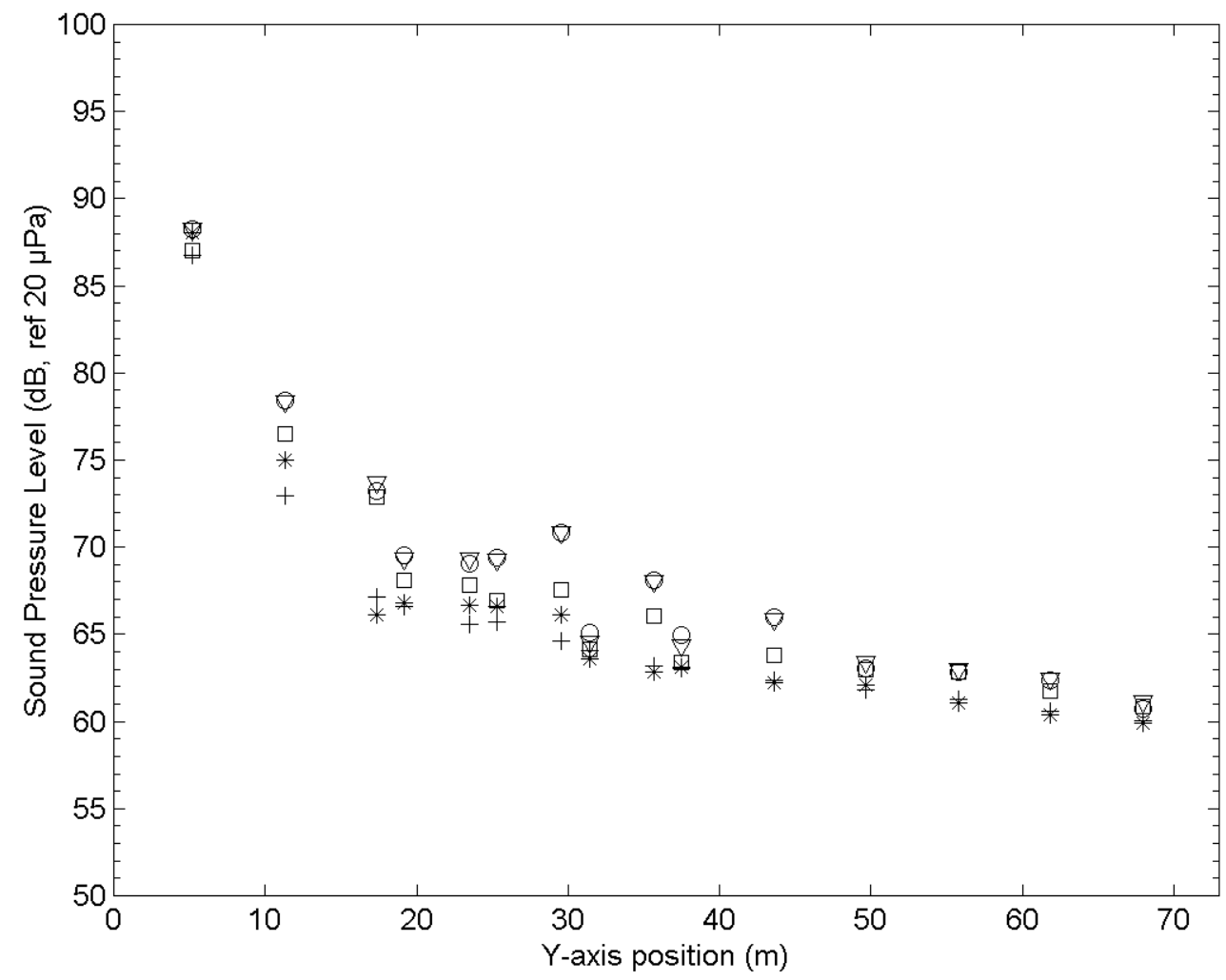

Figure B.62: Overall sound pressure level for sources 91 and 92 as a function of window location. $\nabla$ level of existing toll plaza; * level with absorbing canopy; $\mathrm{O}$ level with absorbing barriers; level with absorbing barriers; + level is all modifications. 


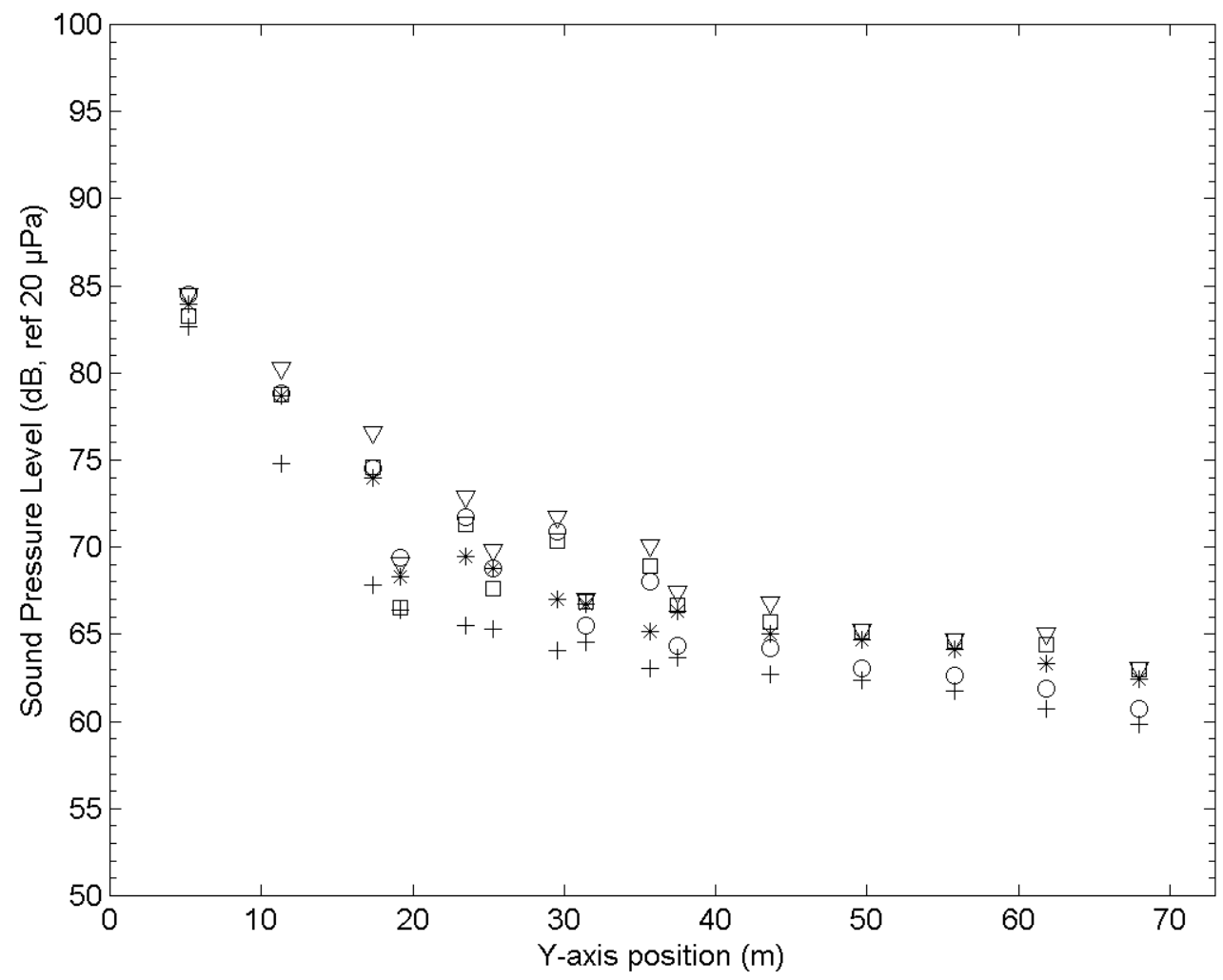

Figure B.63: Overall sound pressure level for sources 93 and 94 as a function of window location. $\nabla$ level of existing toll plaza; * level with absorbing canopy; O level with absorbing barriers; level with absorbing barriers; + level is all modifications. 


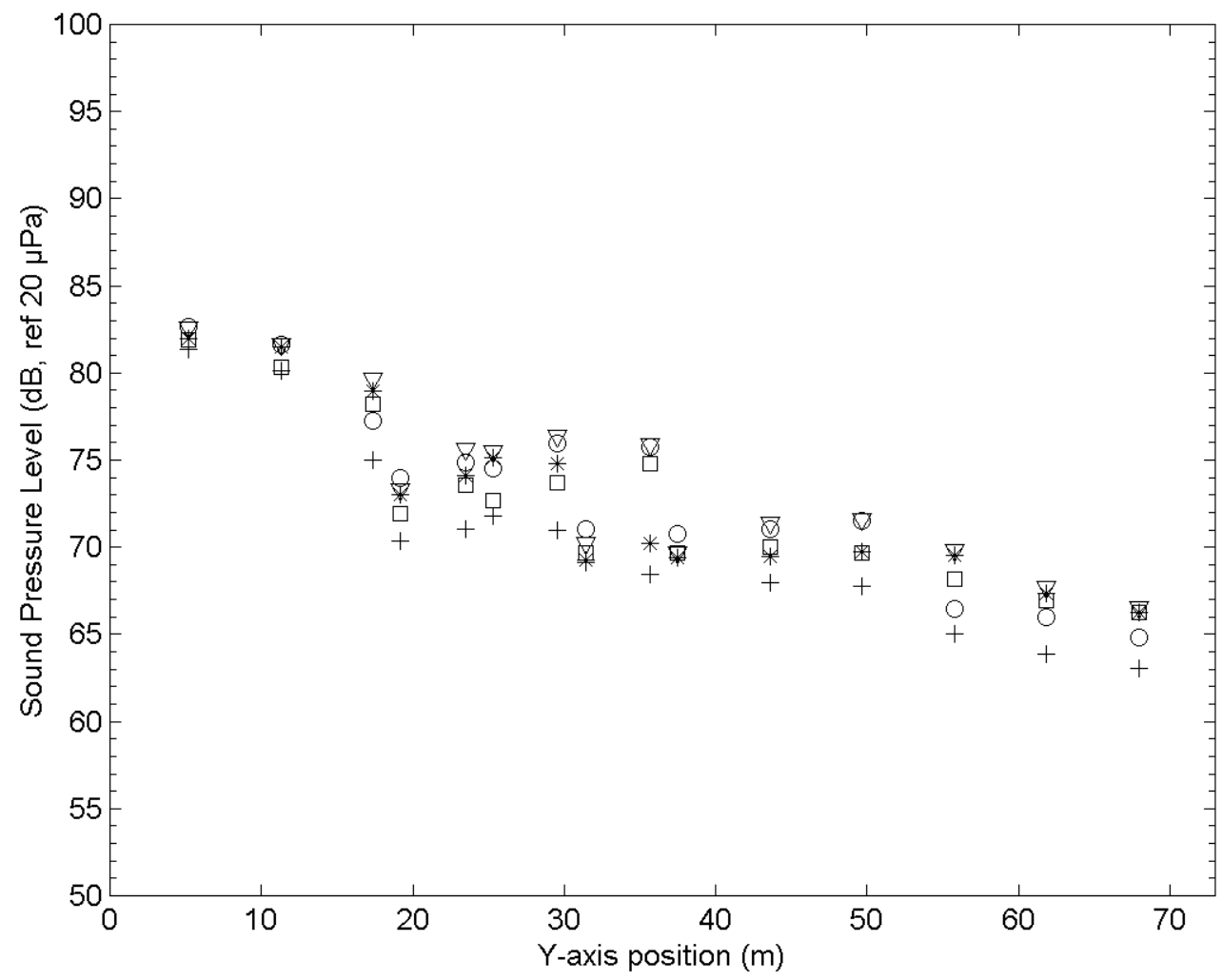

Figure B.64: Overall sound pressure level for sources 95 and 96 as a function of window location. $\nabla$ level of existing toll plaza; * level with absorbing canopy; O level with absorbing barriers; level with absorbing barriers; + level is all modifications. 


\section{B.4 Direct Field Study, Semi-Truck Sources}

To confirm the importance of the direct field, all elements of the model removed expect for the ground. One lane of semi-truck source were activated at a time. The overall level at the for test model was compared to the same case of the reference model. The only meaningful information is for window(s) serving the lane with active sources. Each figure represents the response at all window locations for a particular lane of sources. 


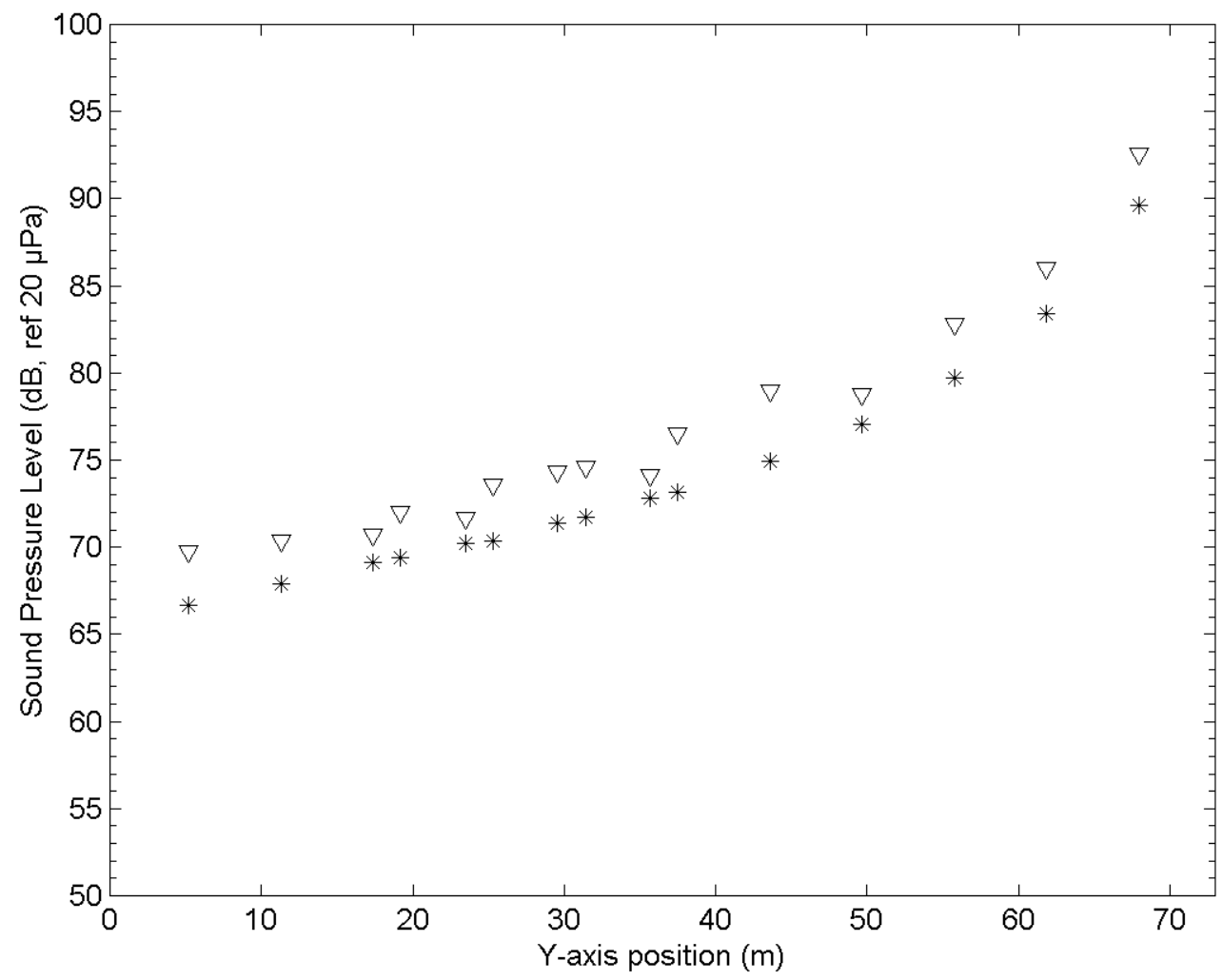

Figure B.65: Overall level for all sources in lane 1, semi-truck sources. $\nabla$ level of existing toll plaza; * level with of direct field validation model. 


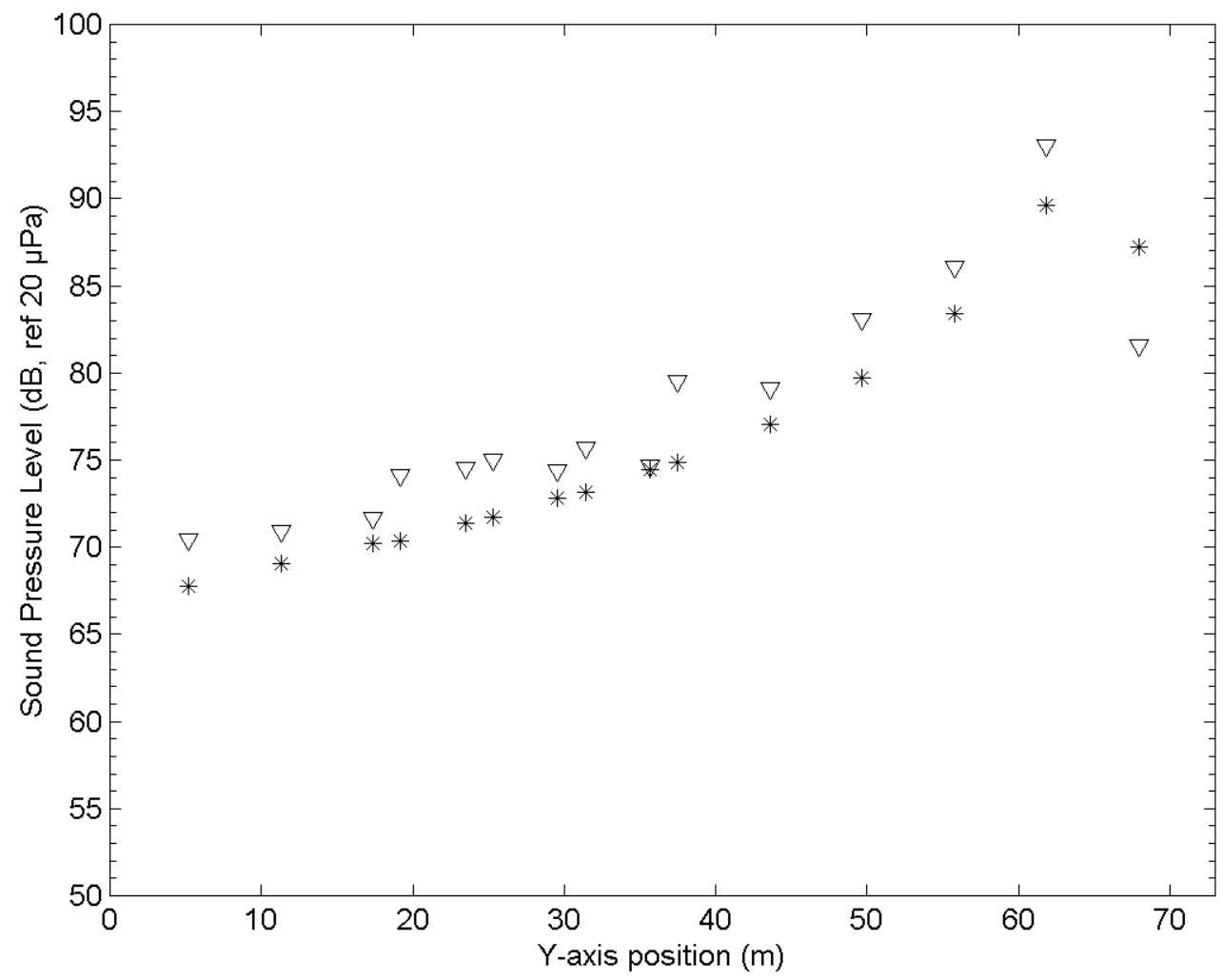

Figure B.66: Overall level for all sources in lane 2, semi-truck sources. $\nabla$ level of existing toll plaza; * level with of direct field validation model. 


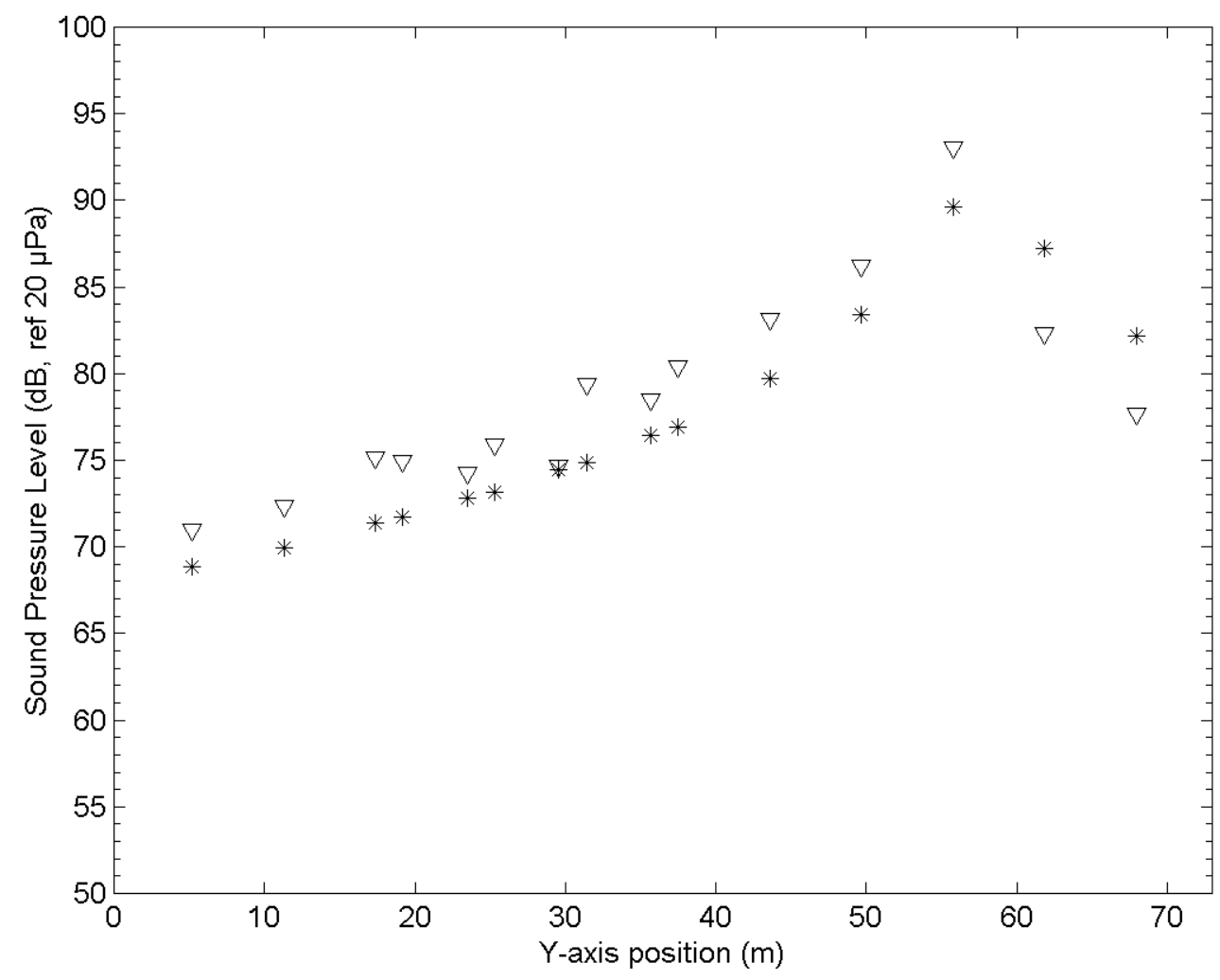

Figure B.67: Overall level for all sources in lane 3, semi-truck sources. $\nabla$ level of existing toll plaza; * level with of direct field validation model. 


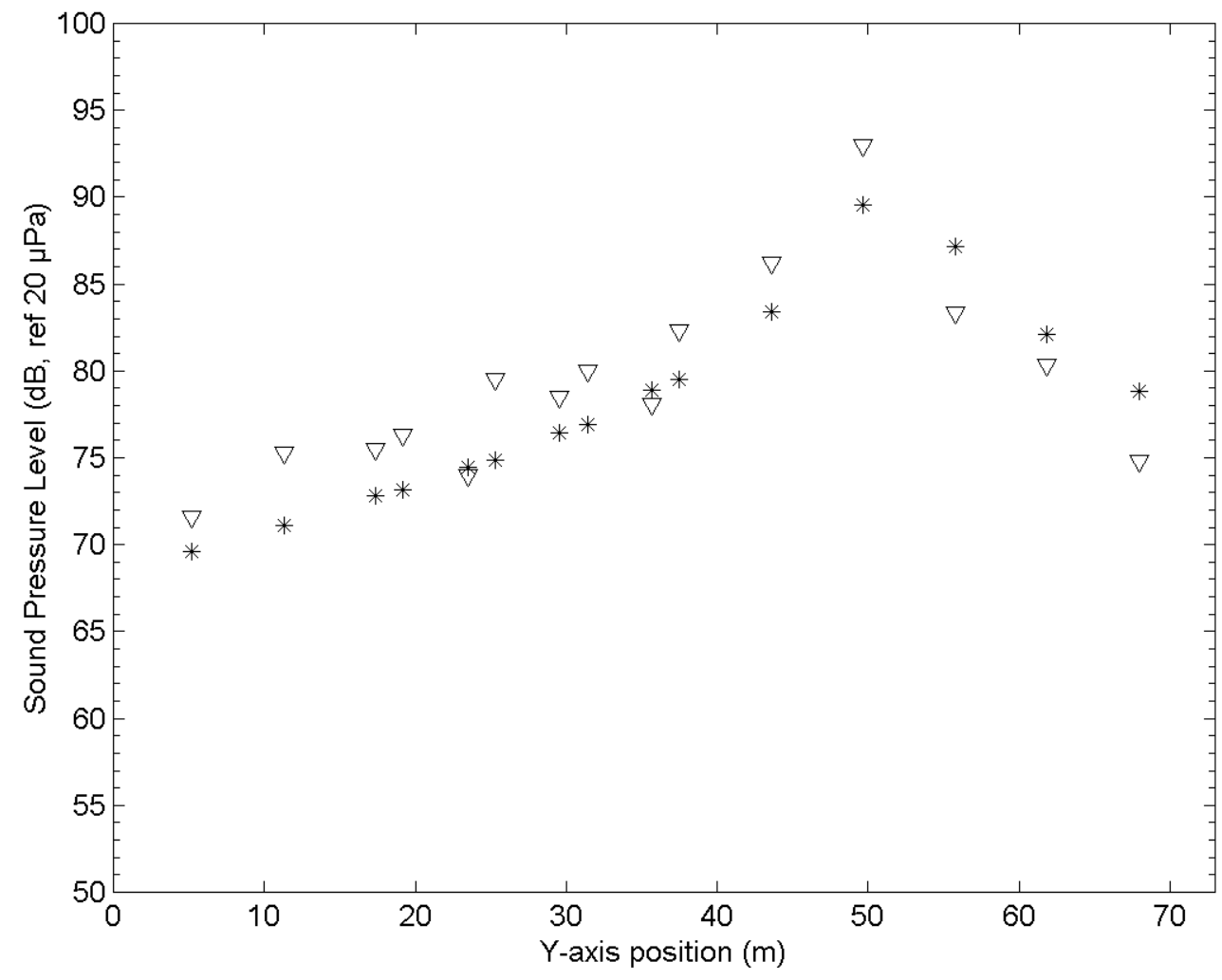

Figure B.68: Overall level for all sources in lane 4, semi-truck sources. $\nabla$ level of existing toll plaza; * level with of direct field validation model. 


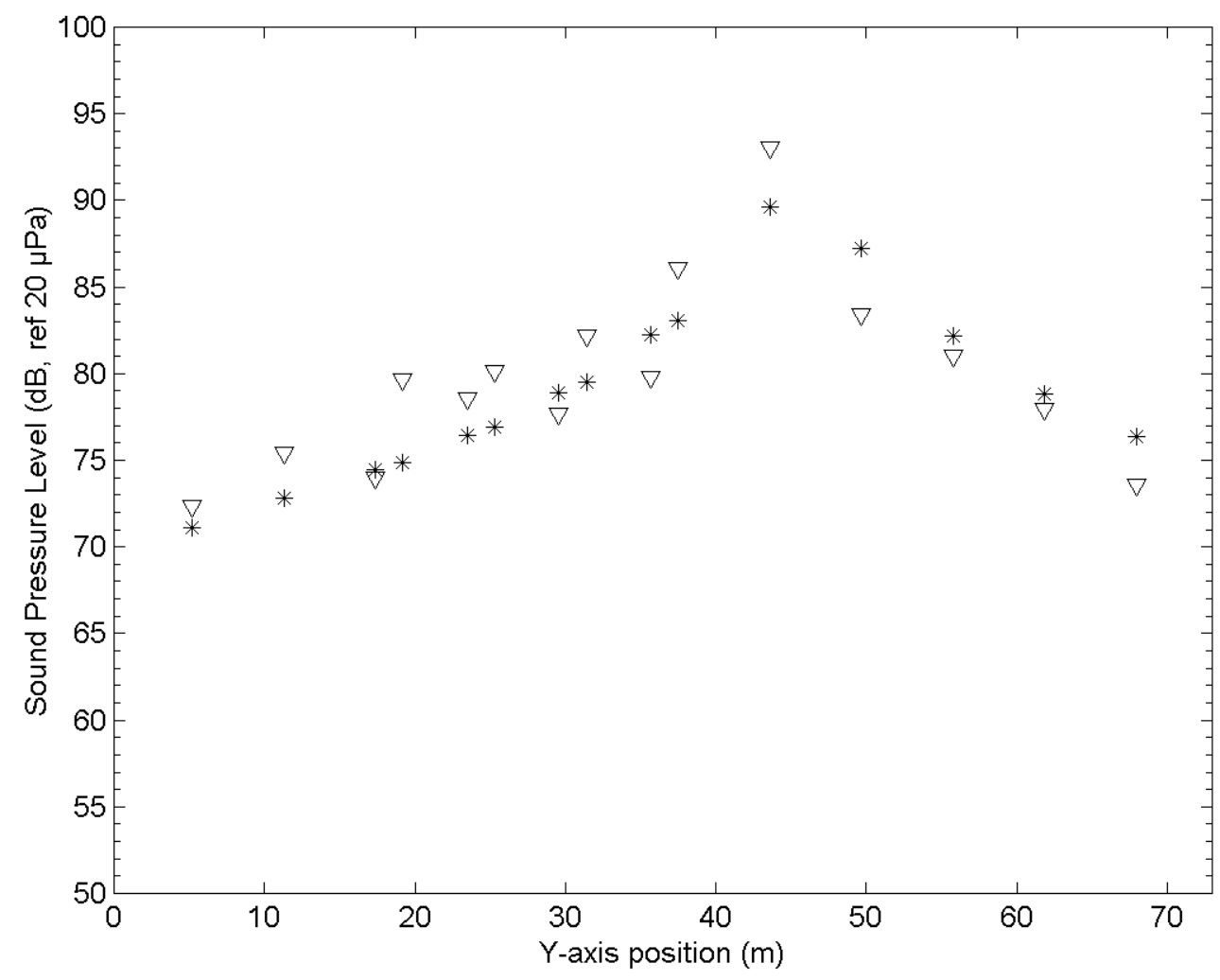

Figure B.69: Overall level for all sources in lane 5, semi-truck sources. $\nabla$ level of existing toll plaza; * level with of direct field validation model. 


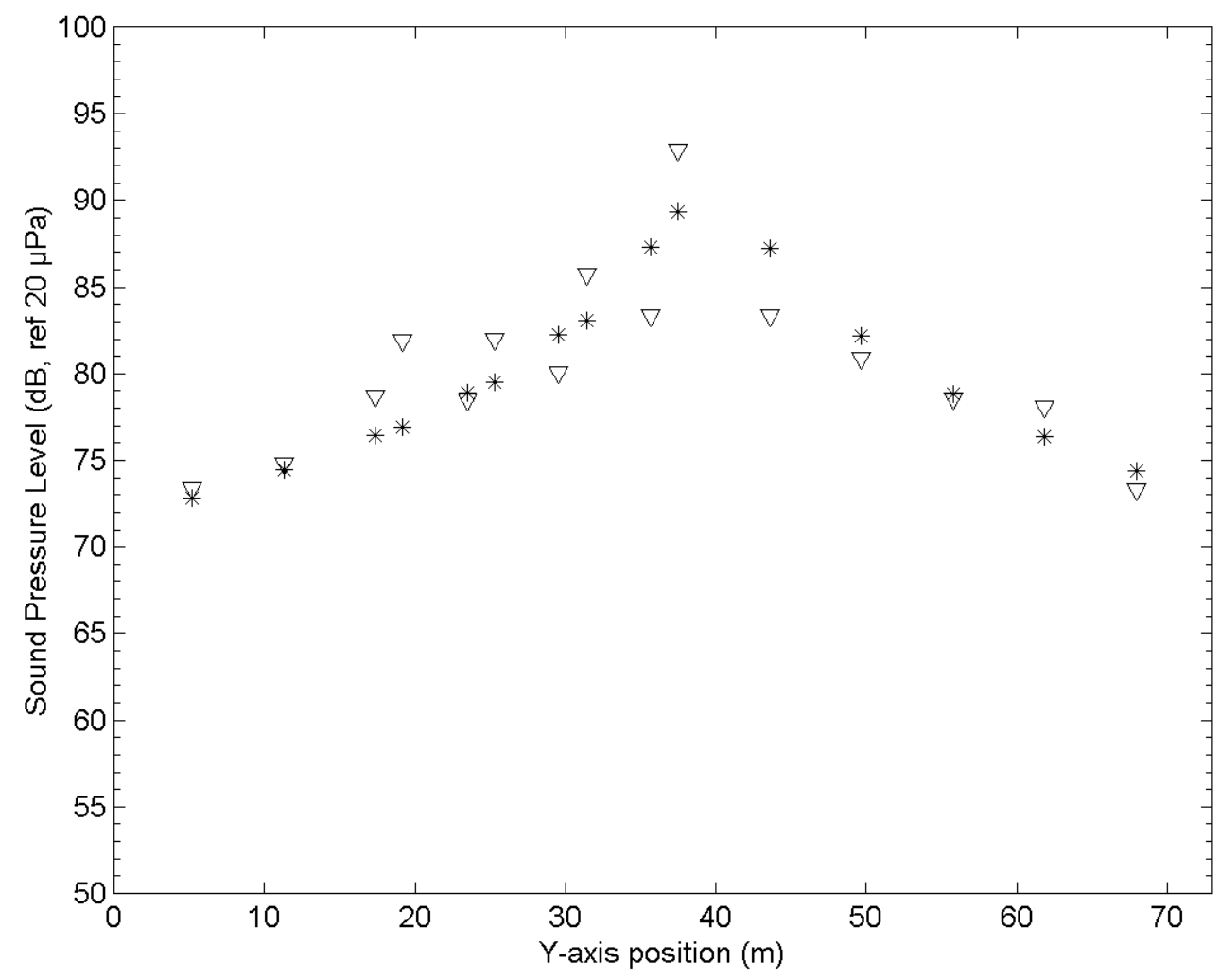

Figure B.70: Overall level for all sources in lane 6 , semi-truck sources. $\nabla$ level of existing toll plaza; * level with of direct field validation model. 


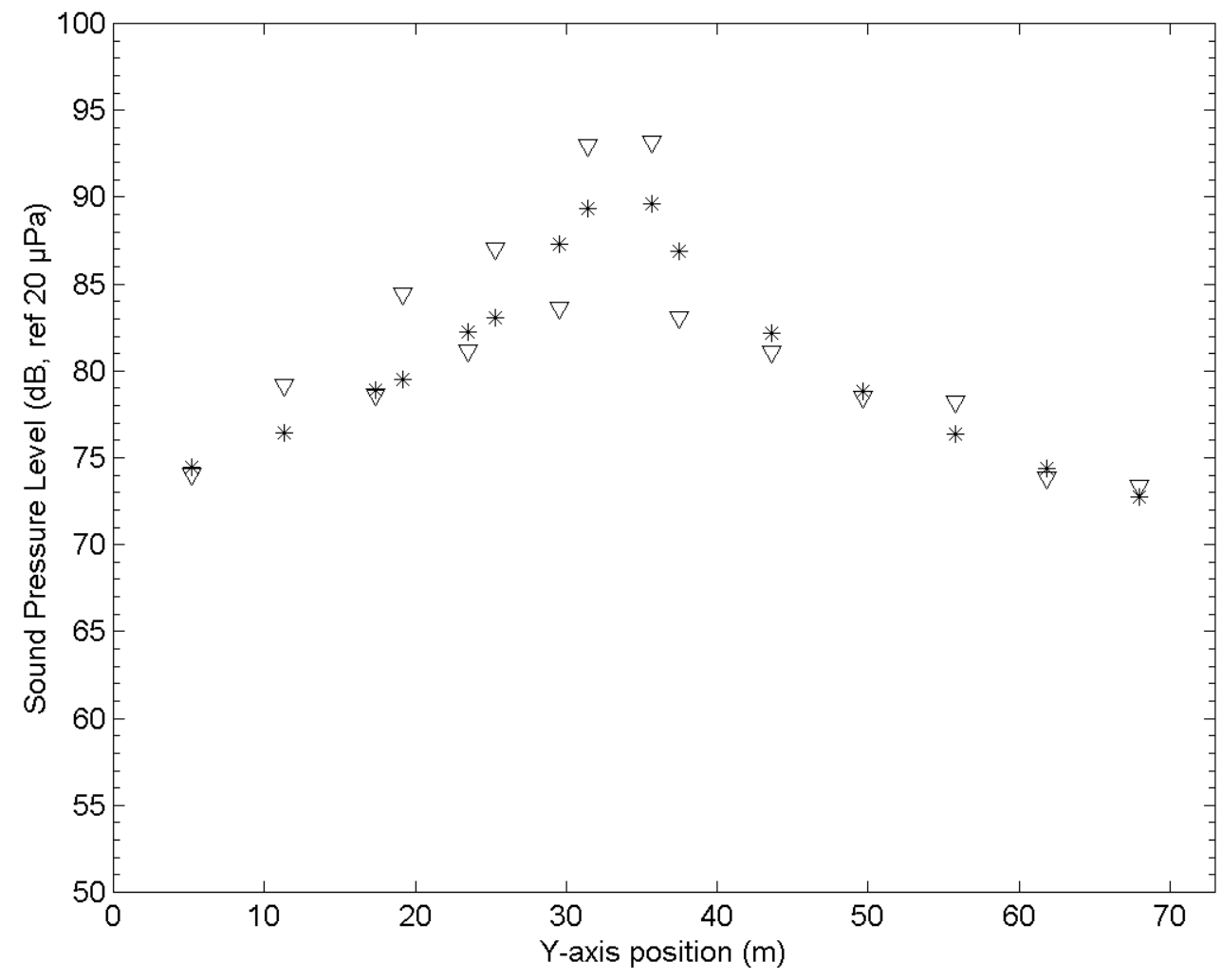

Figure B.71: Overall level for all sources in lane 7, semi-truck sources. $\nabla$ level of existing toll plaza; * level with of direct field validation model. 


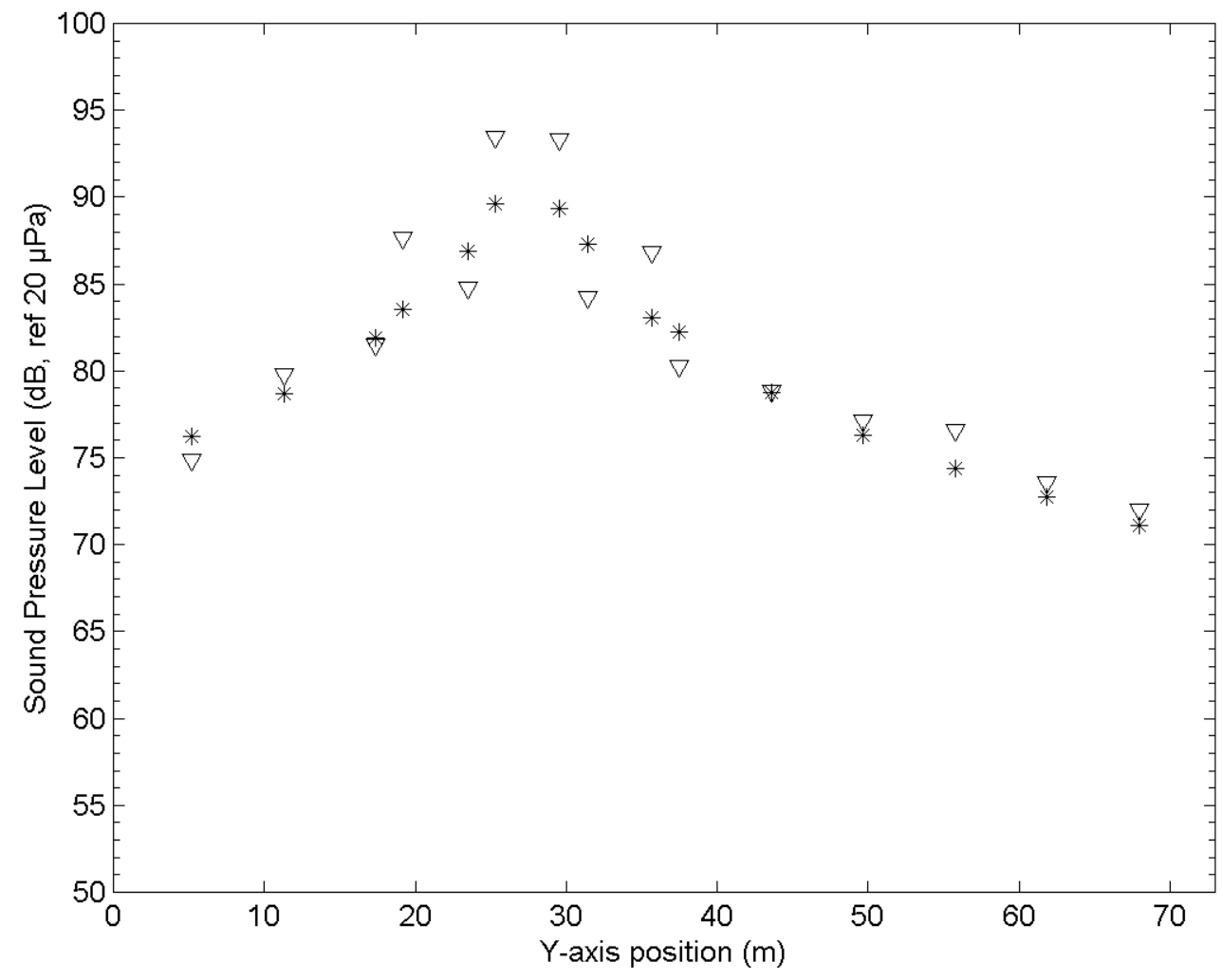

Figure B.72: Overall level for all sources in lane 8, semi-truck sources. $\nabla$ level of existing toll plaza; * level with of direct field validation model. 


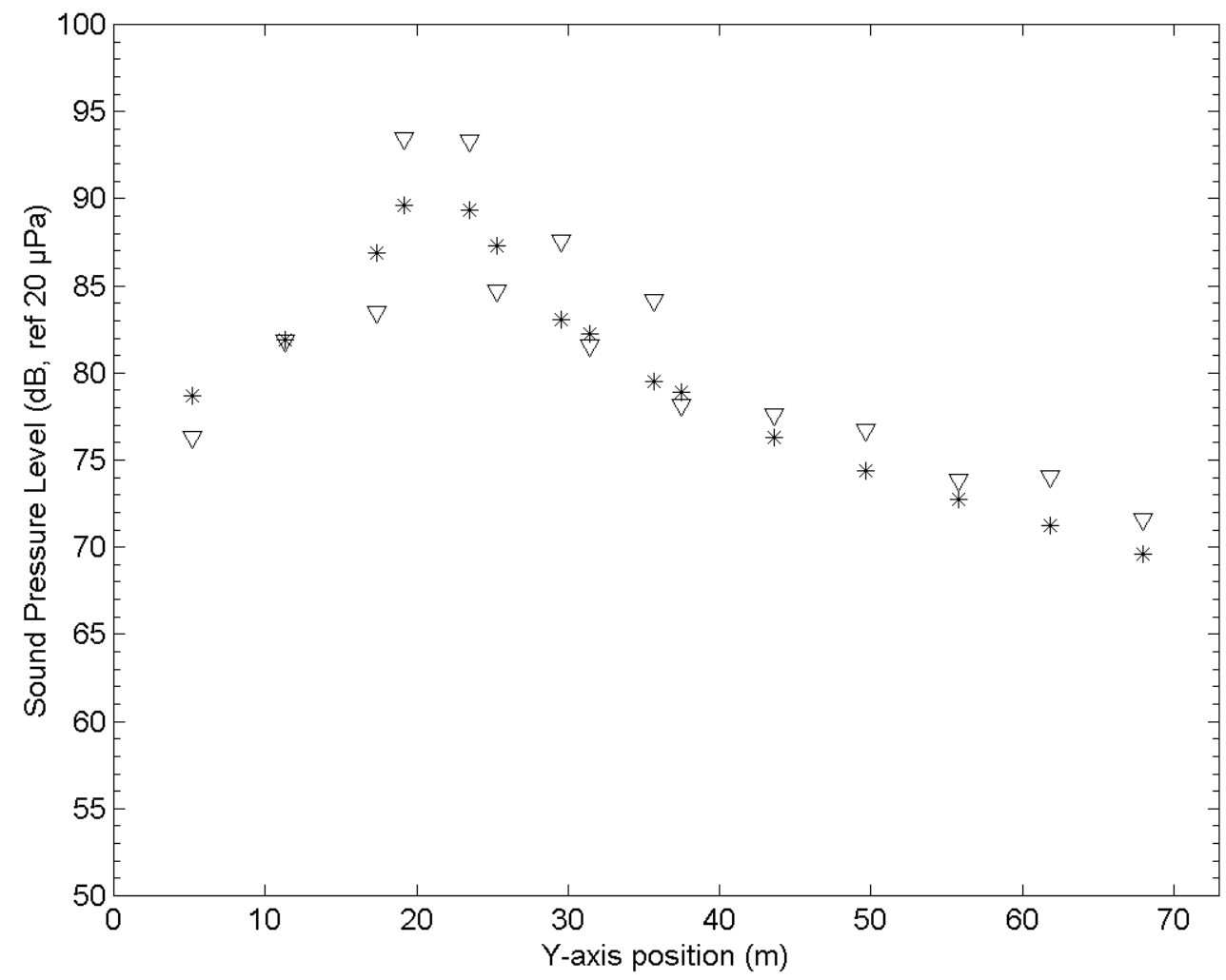

Figure B.73: Overall level for all sources in lane 9, semi-truck sources. $\nabla$ level of existing toll plaza; * level with of direct field validation model. 


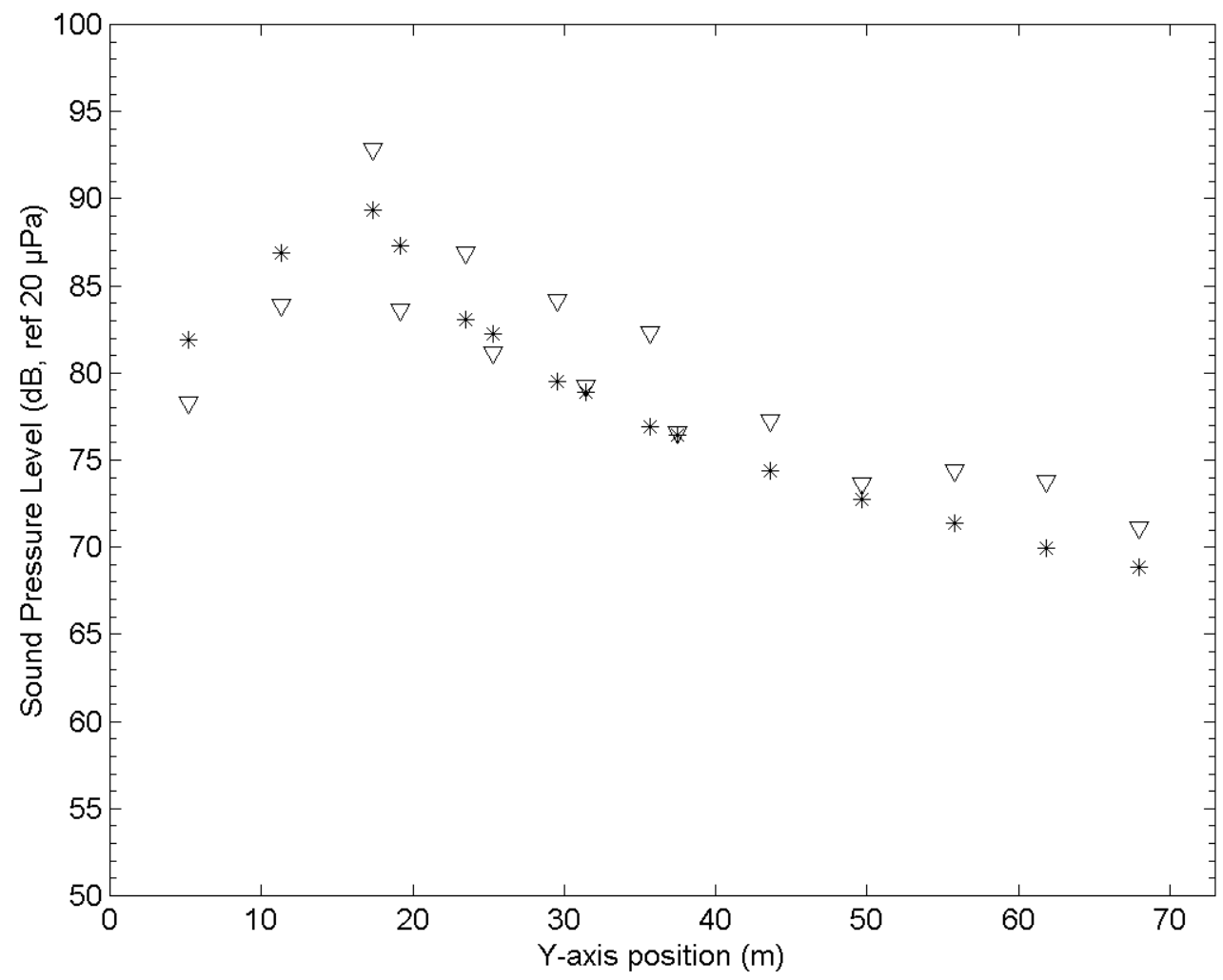

Figure B.74: Overall level for all sources in lane 10, semi-truck sources. $\nabla$ level of existing toll plaza; * level with of direct field validation model. 


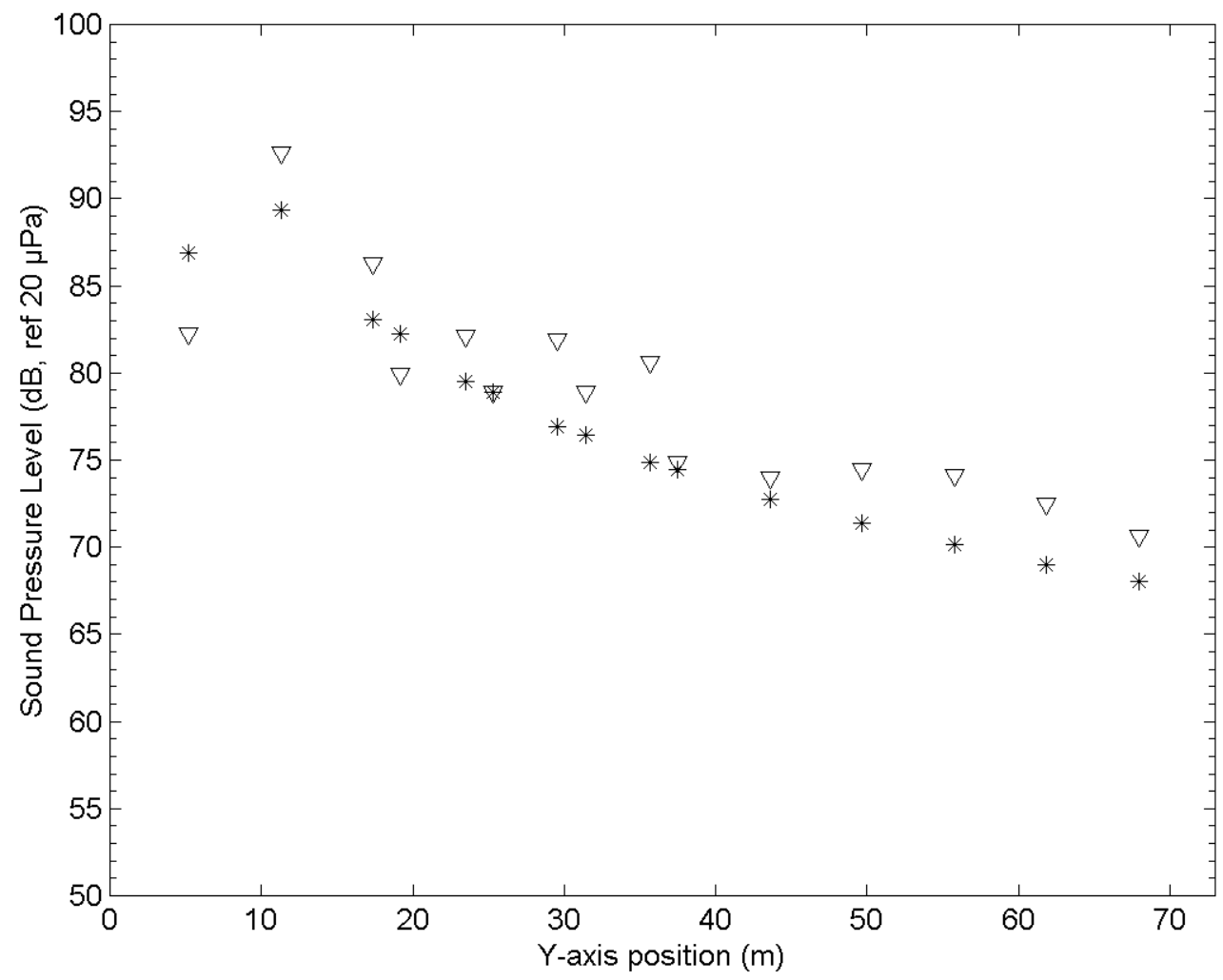

Figure B.75: Overall level for all sources in lane 11, semi-truck sources. $\nabla$ level of existing toll plaza; * level with of direct field validation model. 


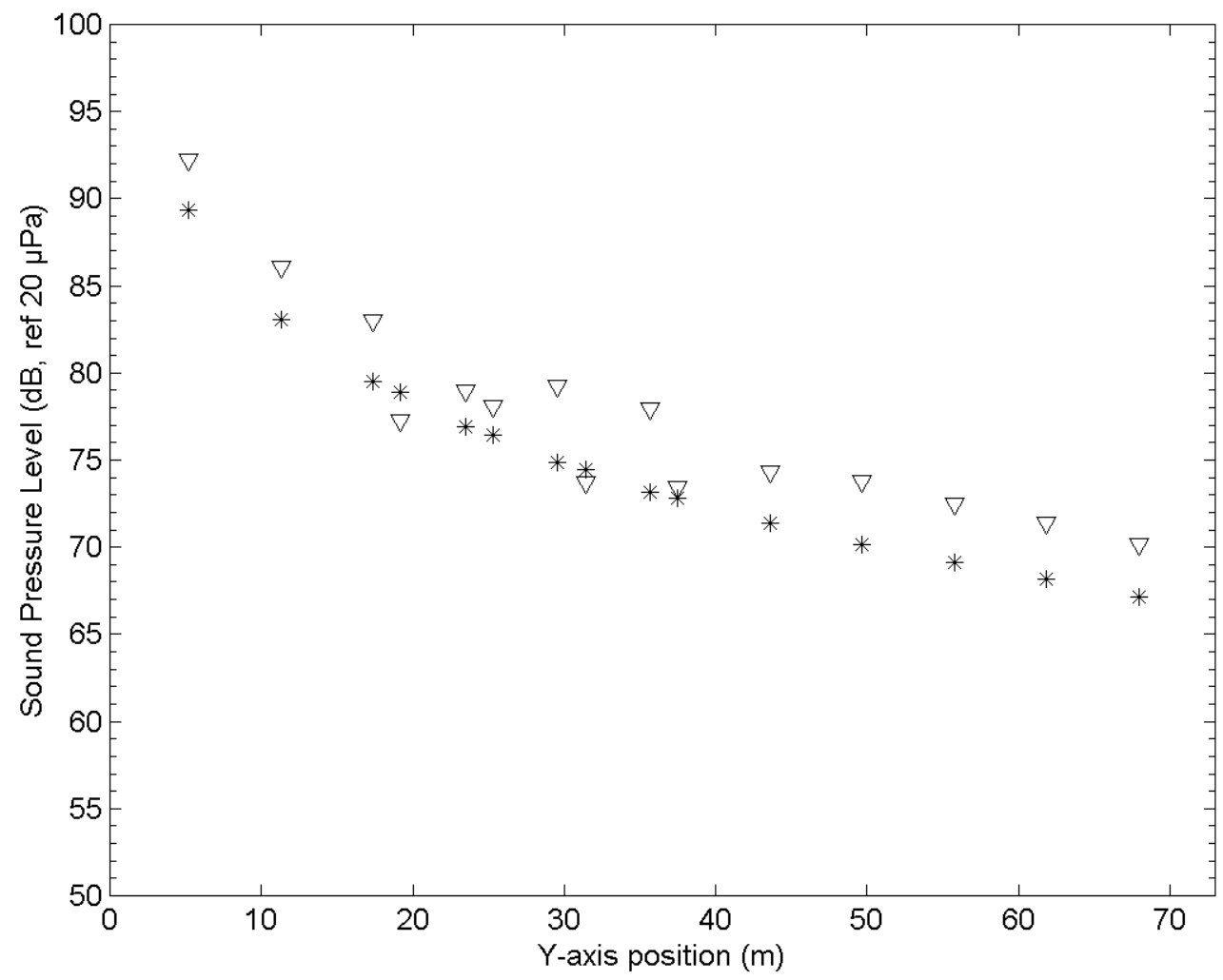

Figure B.76: Overall level for all sources in lane 12, semi-truck sources. $\nabla$ level of existing toll plaza; * level with of direct field validation model. 


\section{B.5 Direct Field Study, Car Sources}

To confirm the importance of the direct field, all elements of the model removed expect for the ground. One lane of car source were activated at a time. The overall level at the for test model was compared to the same case of the reference model. The only meaningful information is for window(s) serving the lane with active sources. Each figure represents the response at all window locations for a particular lane of sources. 


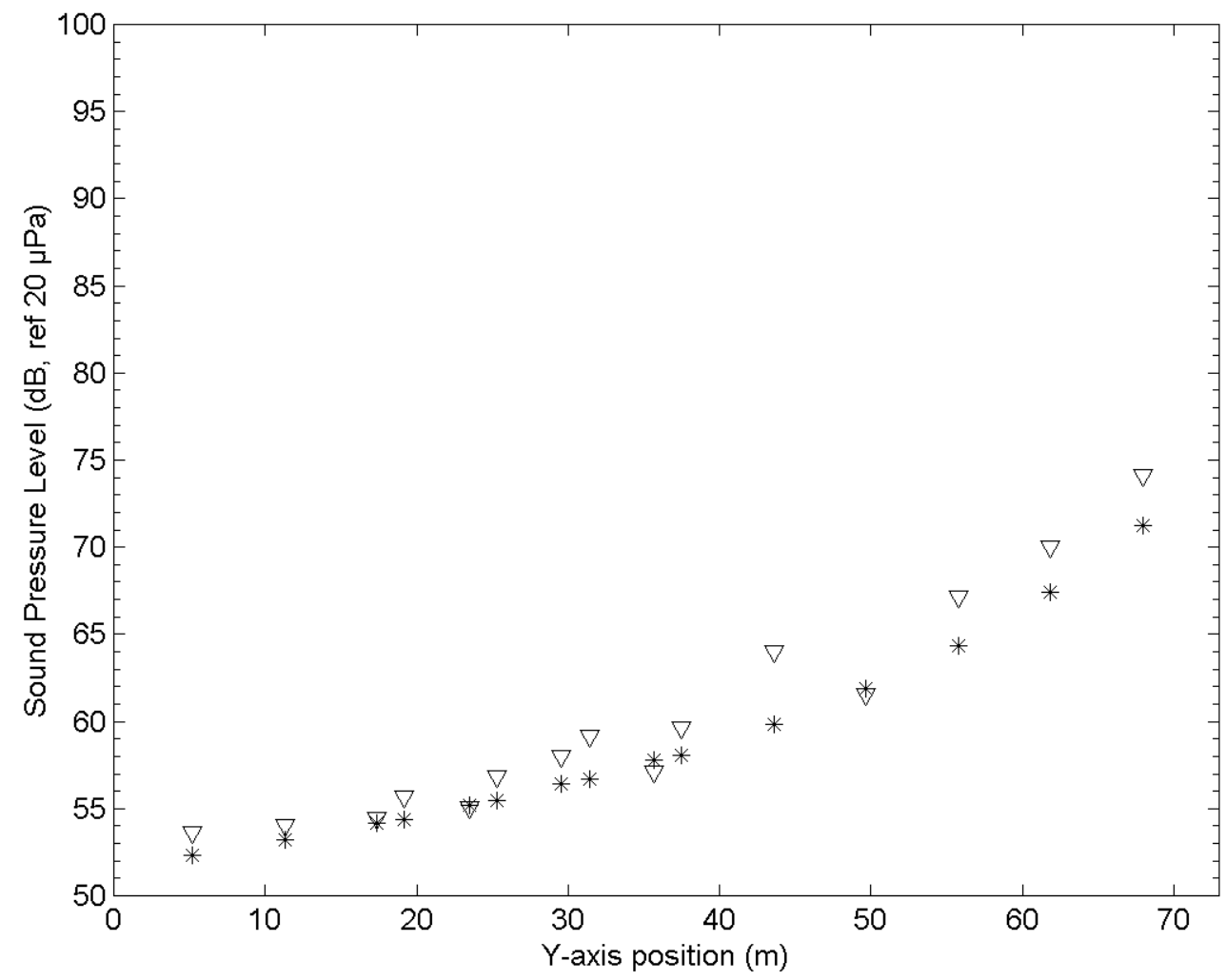

Figure B.77: Overall level for all sources in lane 1, car sources. $\nabla$ level of existing toll plaza; * level with of direct field validation model. 


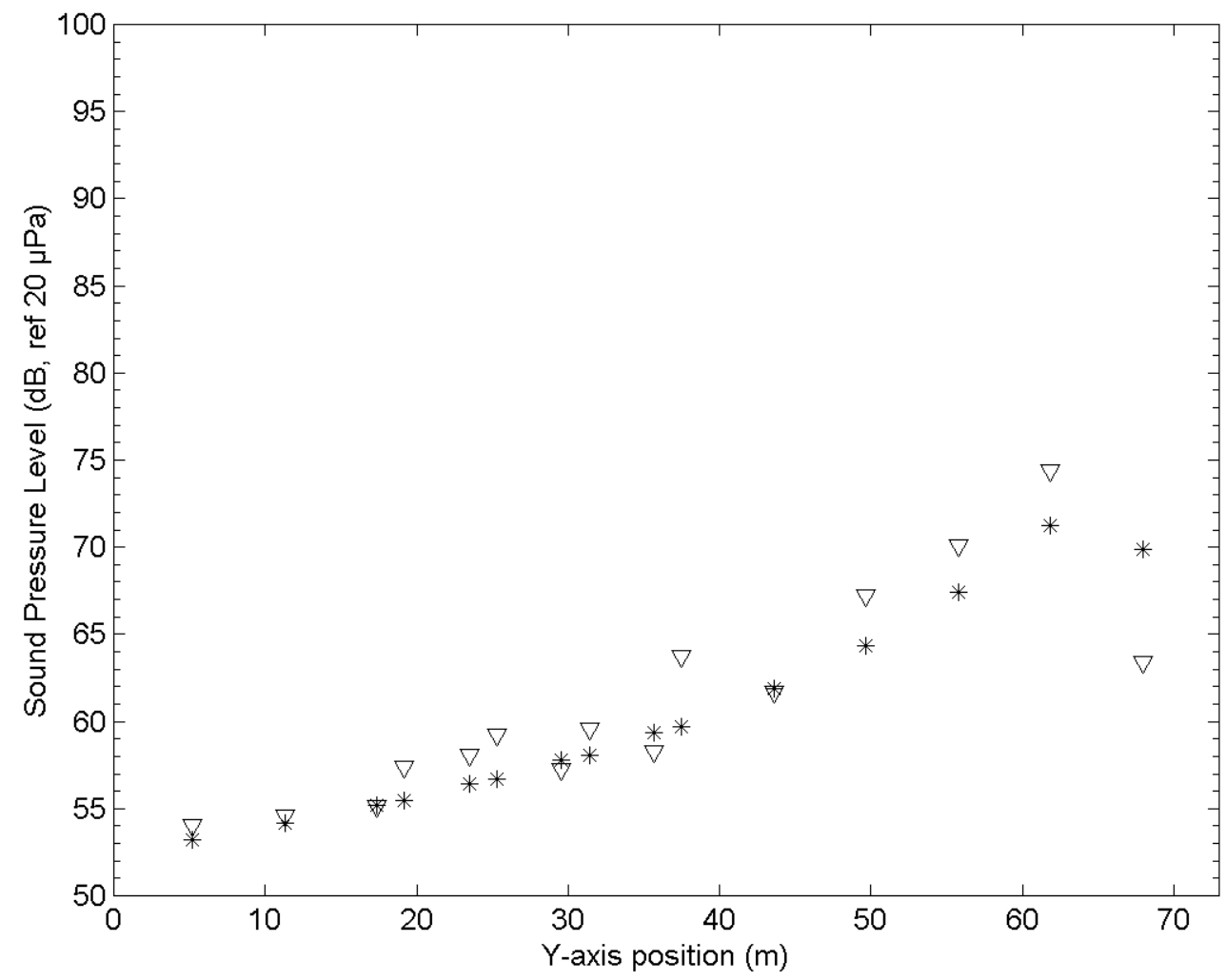

Figure B.78: Overall level for all sources in lane 2, car sources. $\nabla$ level of existing toll plaza; * level with of direct field validation model. 


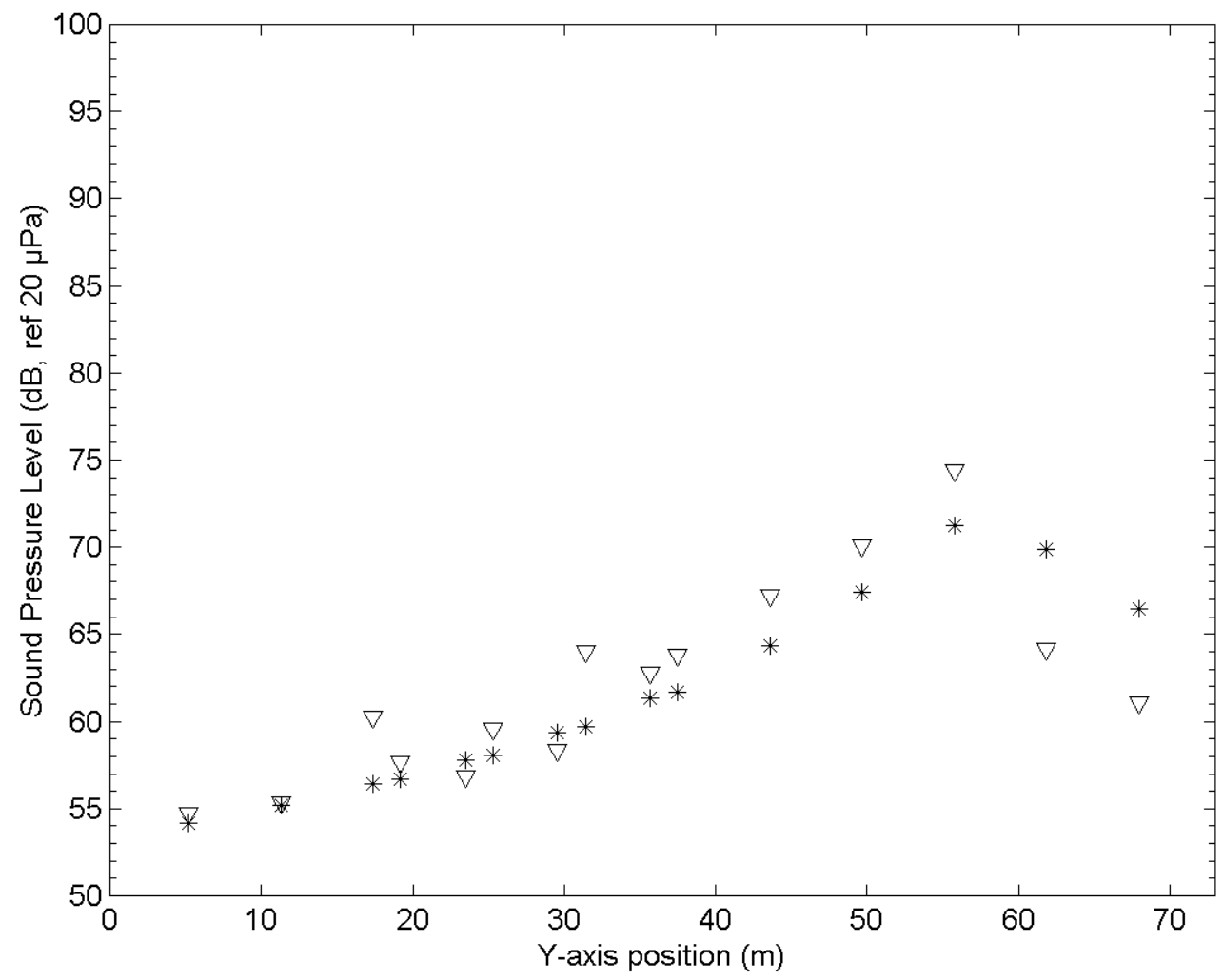

Figure B.79: Overall level for all sources in lane 3, car sources. $\nabla$ level of existing toll plaza; * level with of direct field validation model. 


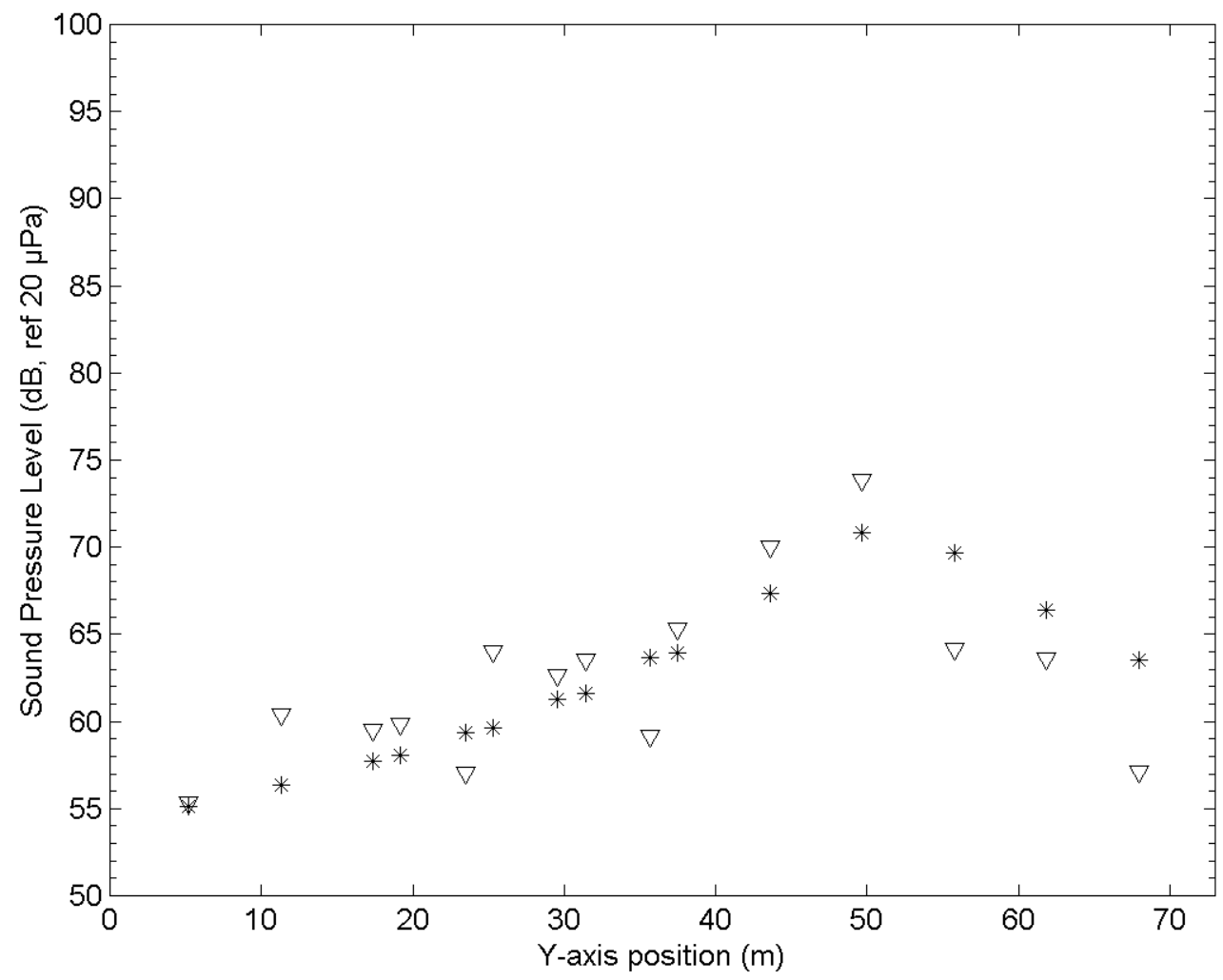

Figure B.80: Overall level for all sources in lane 4, car sources. $\nabla$ level of existing toll plaza; * level with of direct field validation model. 


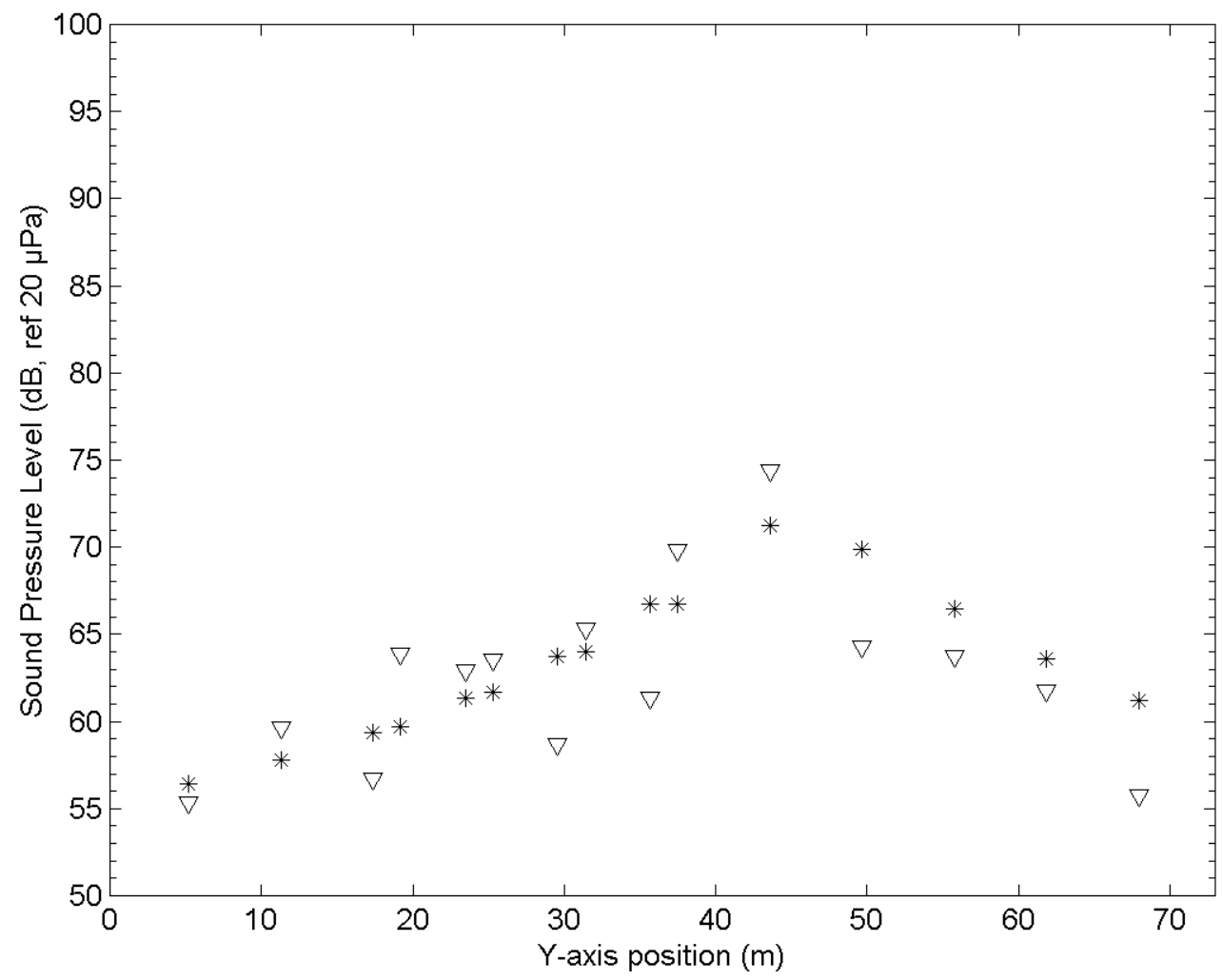

Figure B.81: Overall level for all sources in lane 5, car sources. $\nabla$ level of existing toll plaza; * level with of direct field validation model. 


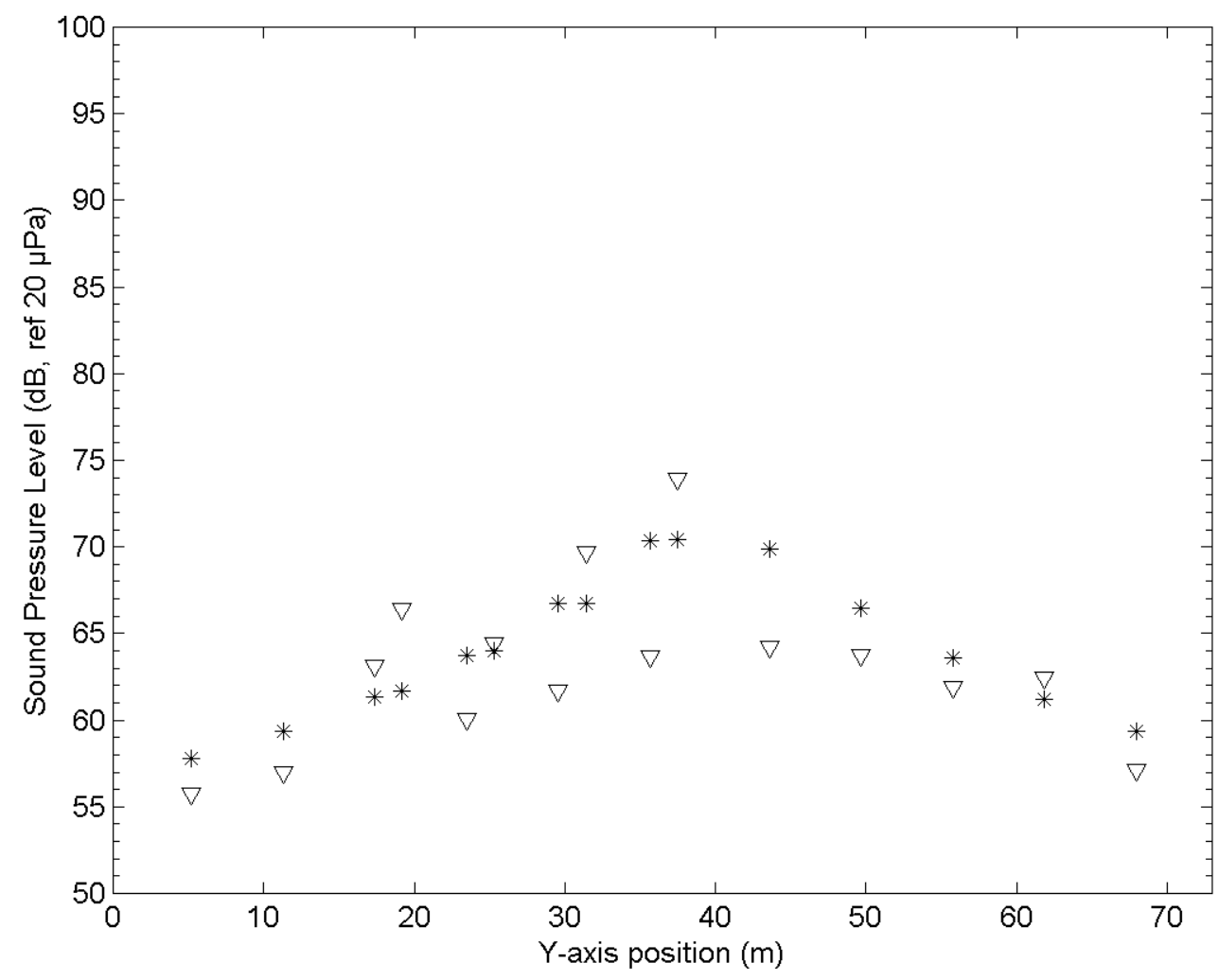

Figure B.82: Overall level for all sources in lane 6, car sources. $\nabla$ level of existing toll plaza; * level with of direct field validation model. 


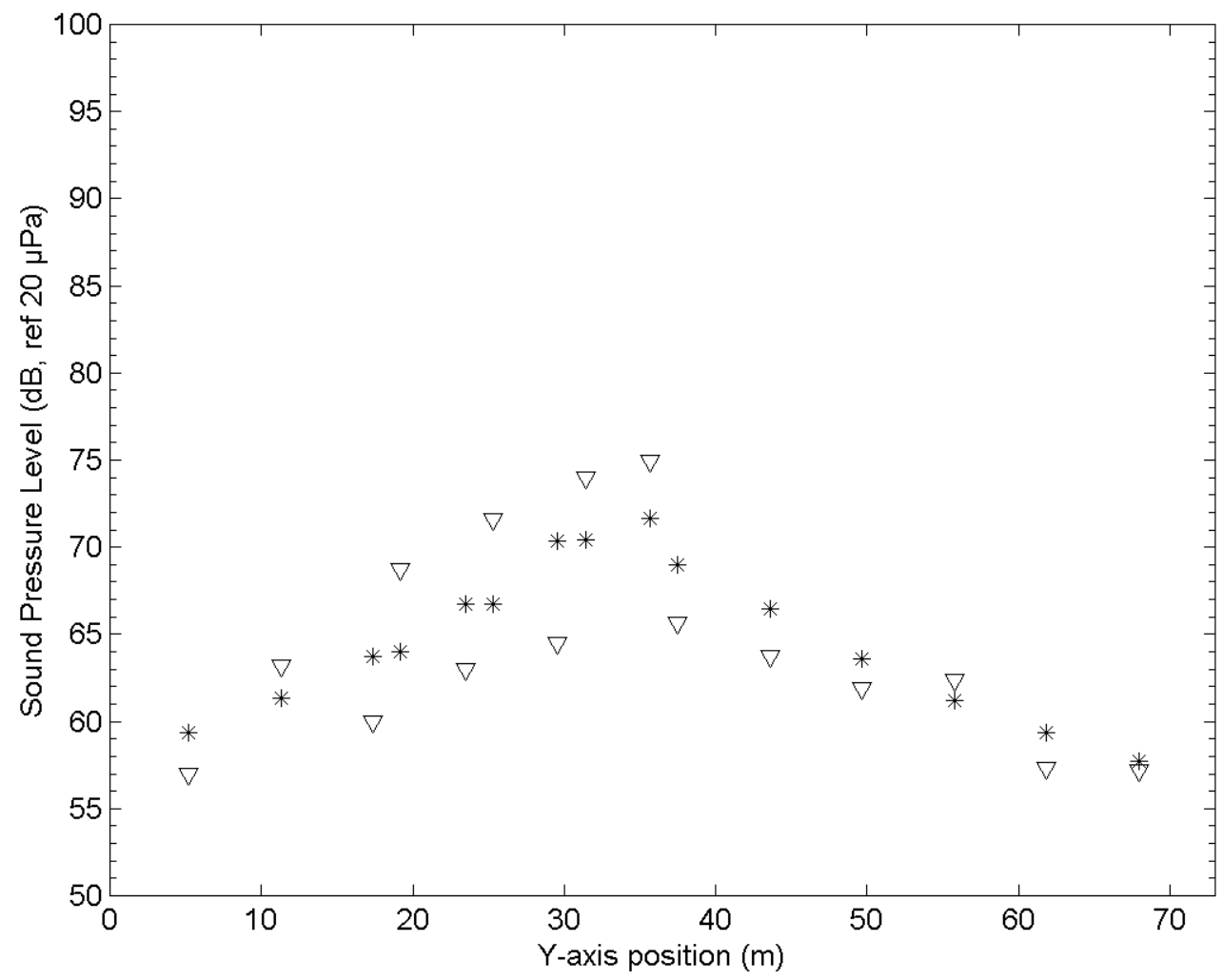

Figure B.83: Overall level for all sources in lane 7, car sources. $\nabla$ level of existing toll plaza; * level with of direct field validation model. 


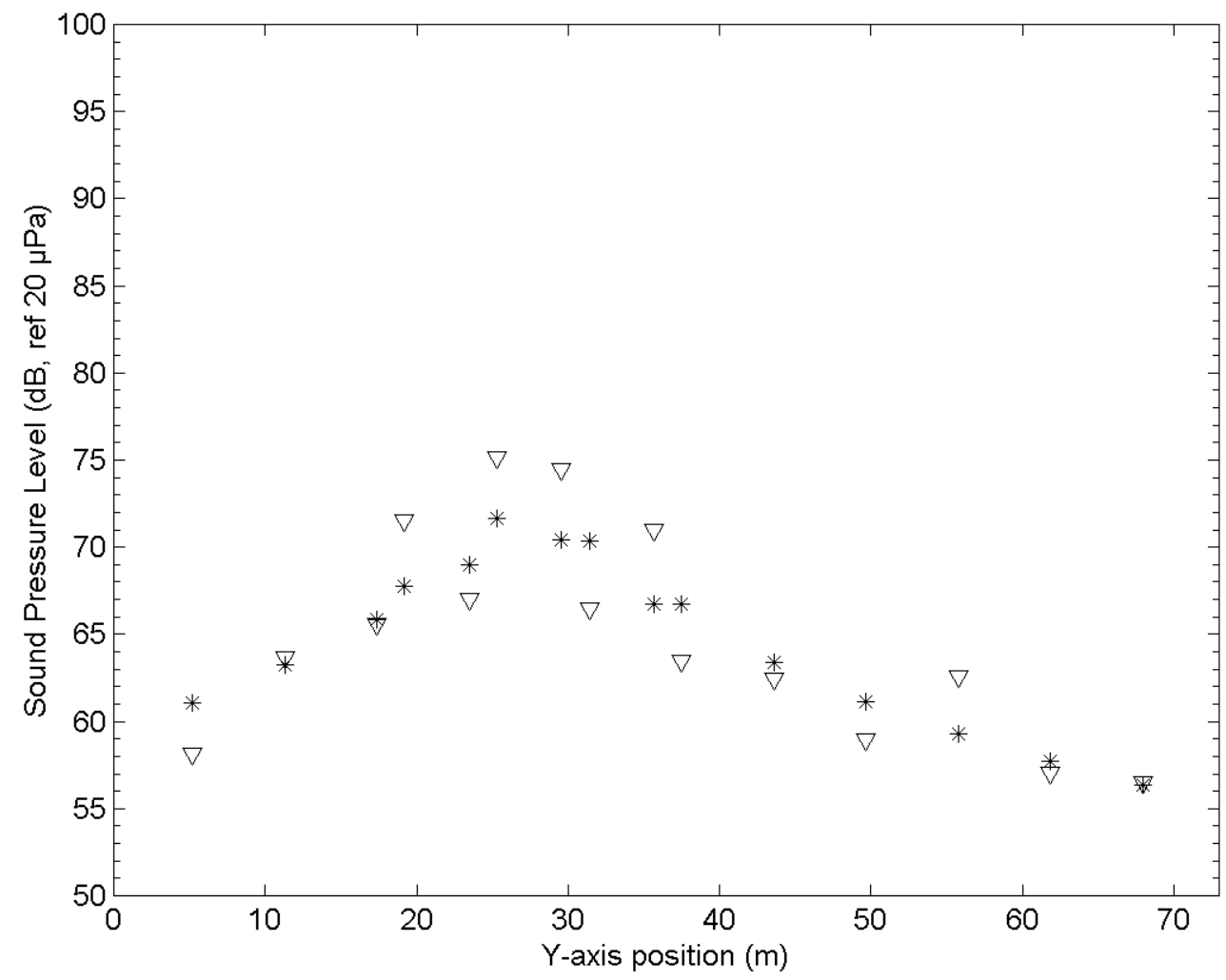

Figure B.84: Overall level for all sources in lane 8, car sources. $\nabla$ level of existing toll plaza; * level with of direct field validation model. 


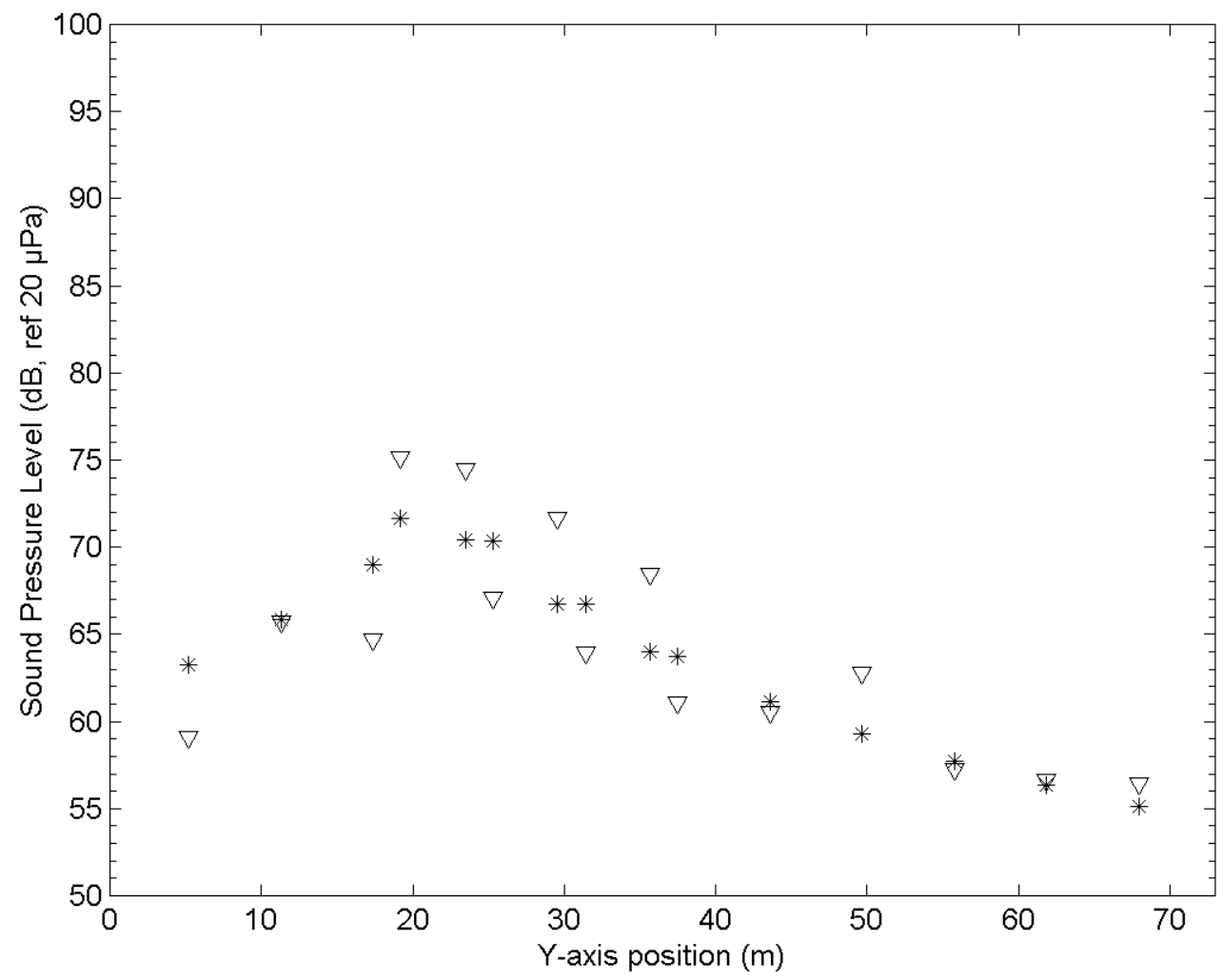

Figure B.85: Overall level for all sources in lane 9, car sources. $\nabla$ level of existing toll plaza; * level with of direct field validation model. 


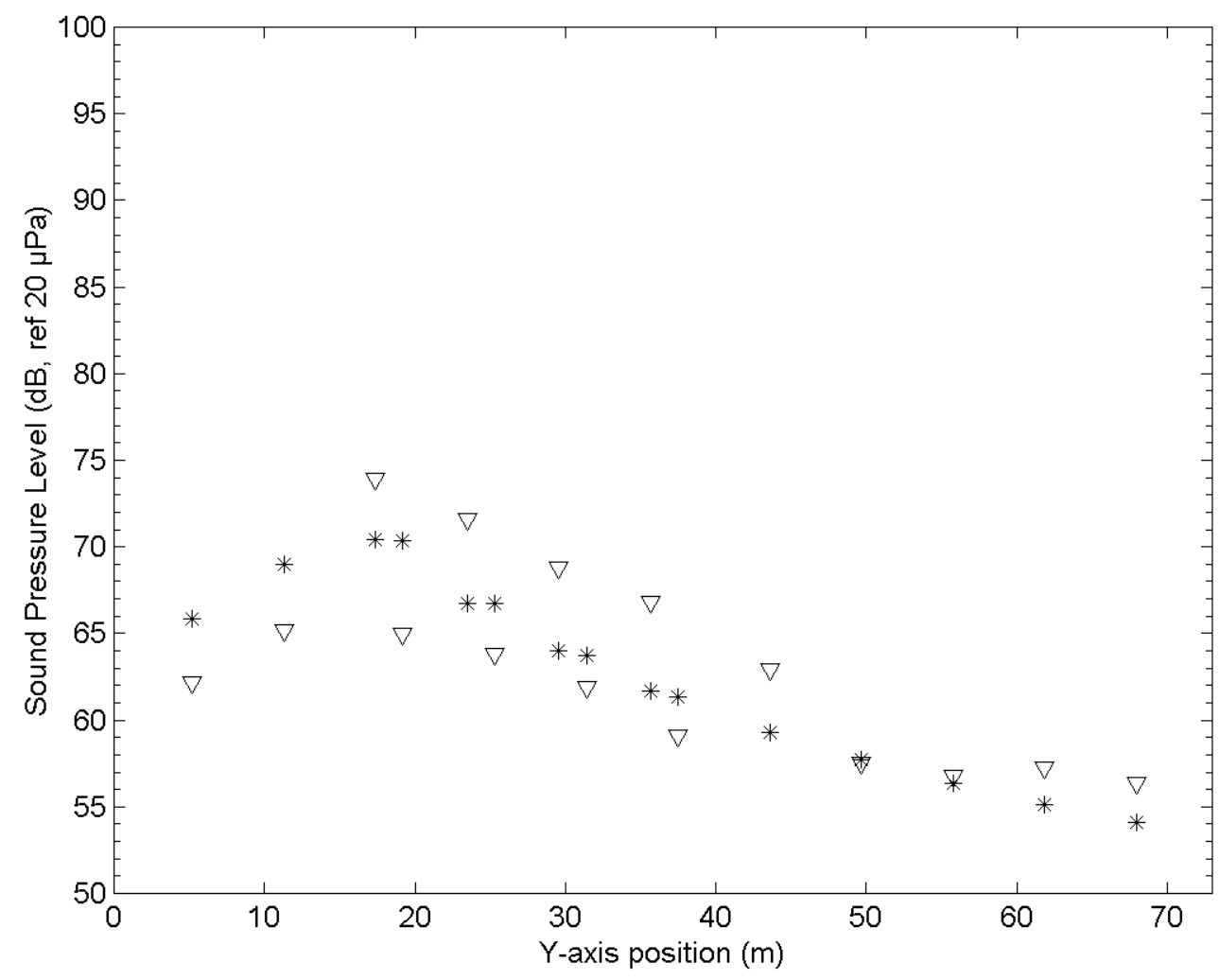

Figure B.86: Overall level for all sources in lane 10, car sources. $\nabla$ level of existing toll plaza; * level with of direct field validation model. 


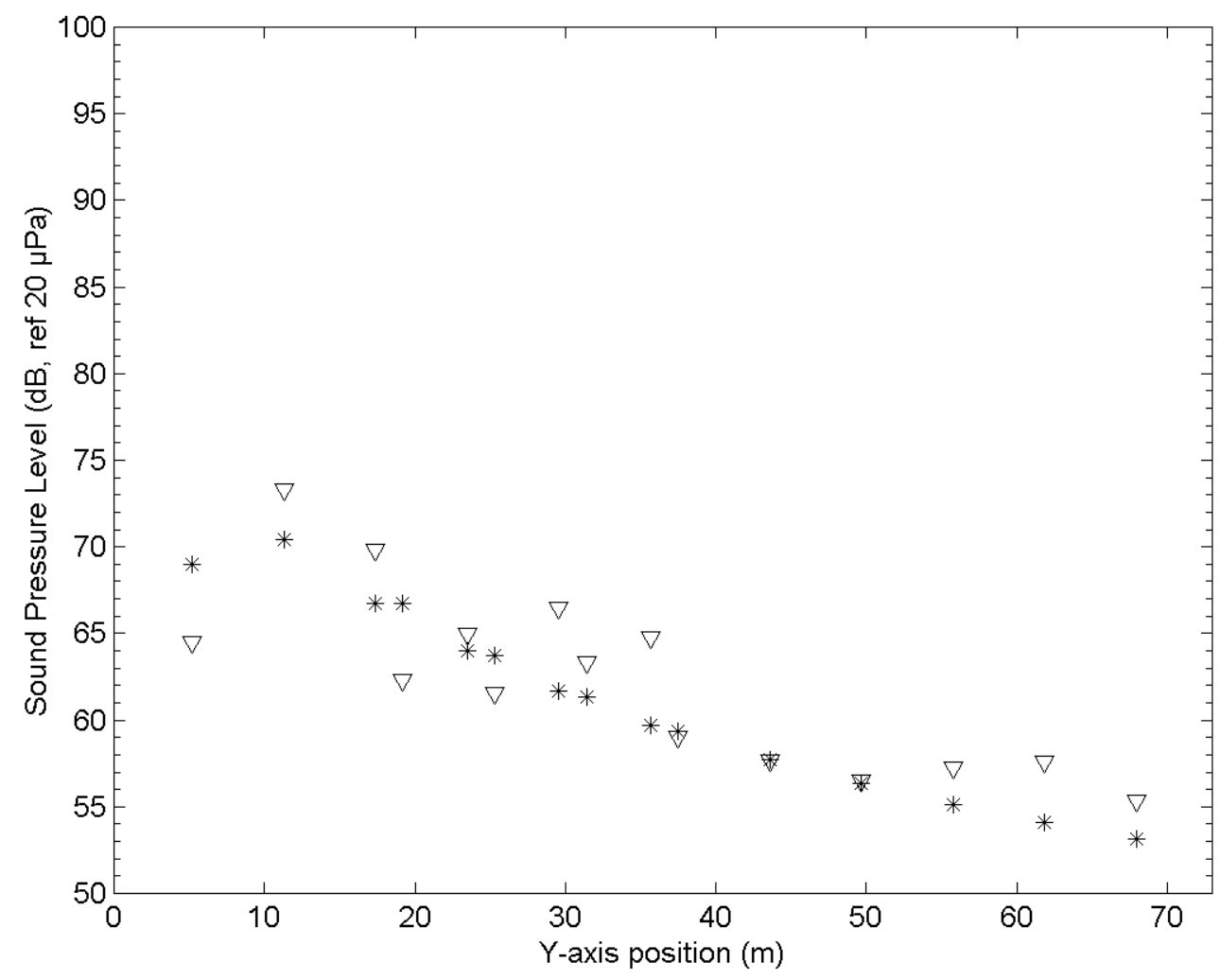

Figure B.87: Overall level for all sources in lane 11, car sources. $\nabla$ level of existing toll plaza; * level with of direct field validation model. 


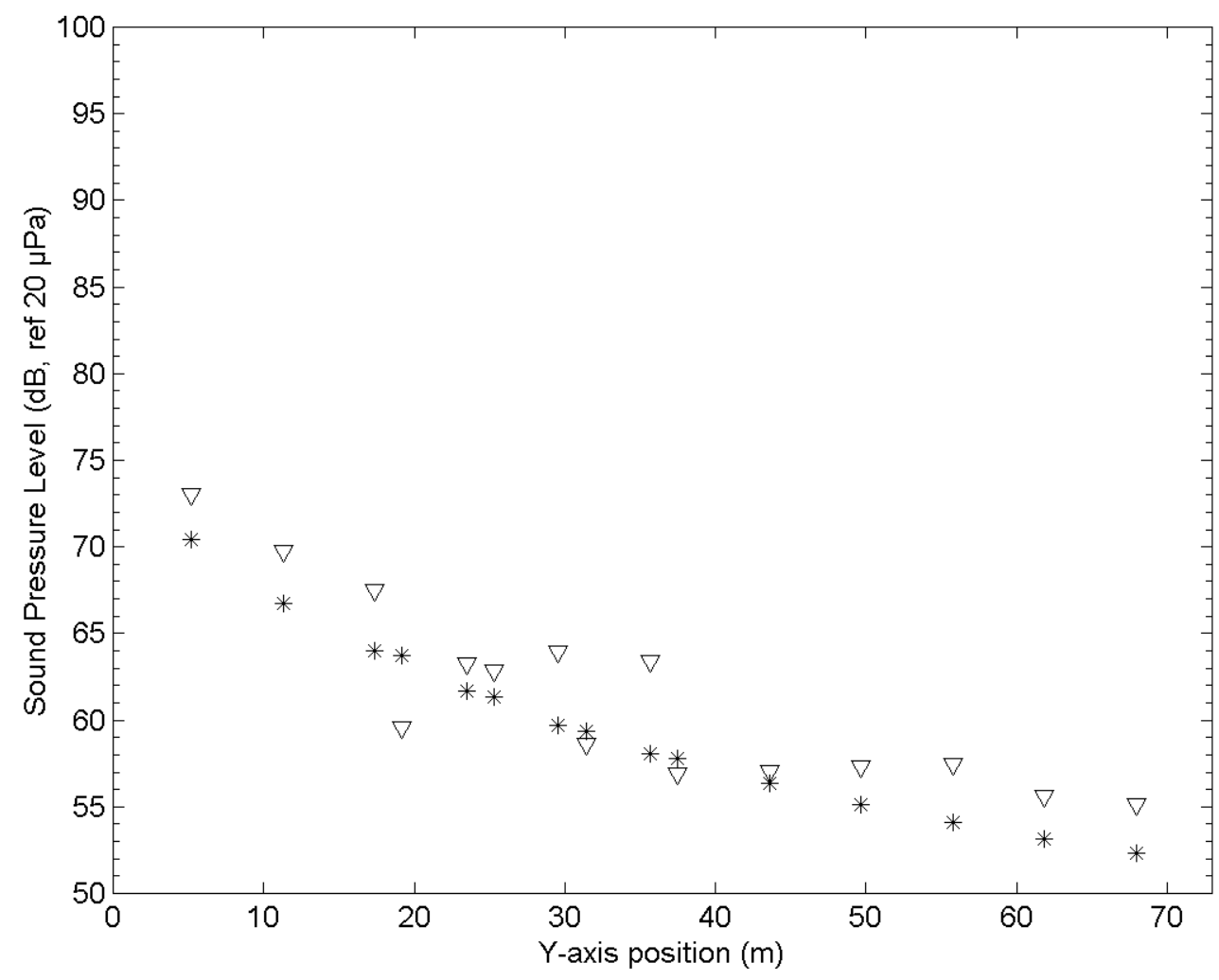

Figure B.88: Overall level for all sources in lane 12, car sources. $\nabla$ level of existing toll plaza; * level with of direct field validation model. 